In cooperation with the U.S. ENVIRONMENTAL PROTECTION AGENCY

Water-Quality, Bed-Sediment, and Biological Data (October 1998 Through September 1999) and Statistical Summaries of Data for Streams in the Upper Clark Fork Basin, Montana

By Kent A. Dodge, Michelle I. Hornberger, and Carlos Primo C. David

Open-File Report 00-370

U.S. Department of the Interior U.S. Geological Survey 


\section{U.S. Department of the Interior}

BRUCE BABBITT, Secretary

\section{U.S. Geological Survey}

Charles G. Groat, Director

Any use of trade, product, or firm names in this publication is for descriptive purposes only and does not imply endorsement by the U.S. Government

Helena, Montana

September 2000

For additional information write to:

\section{District Chief}

U.S. Geological Survey

3162 Bozeman Avenue

Helena, MT 59601-6456

Copies of this report may be purchased from:

\section{U.S. Geological Survey}

Branch of Information Services

Box 25286

Denver, CO 80225-0286 


\section{CONTENTS}

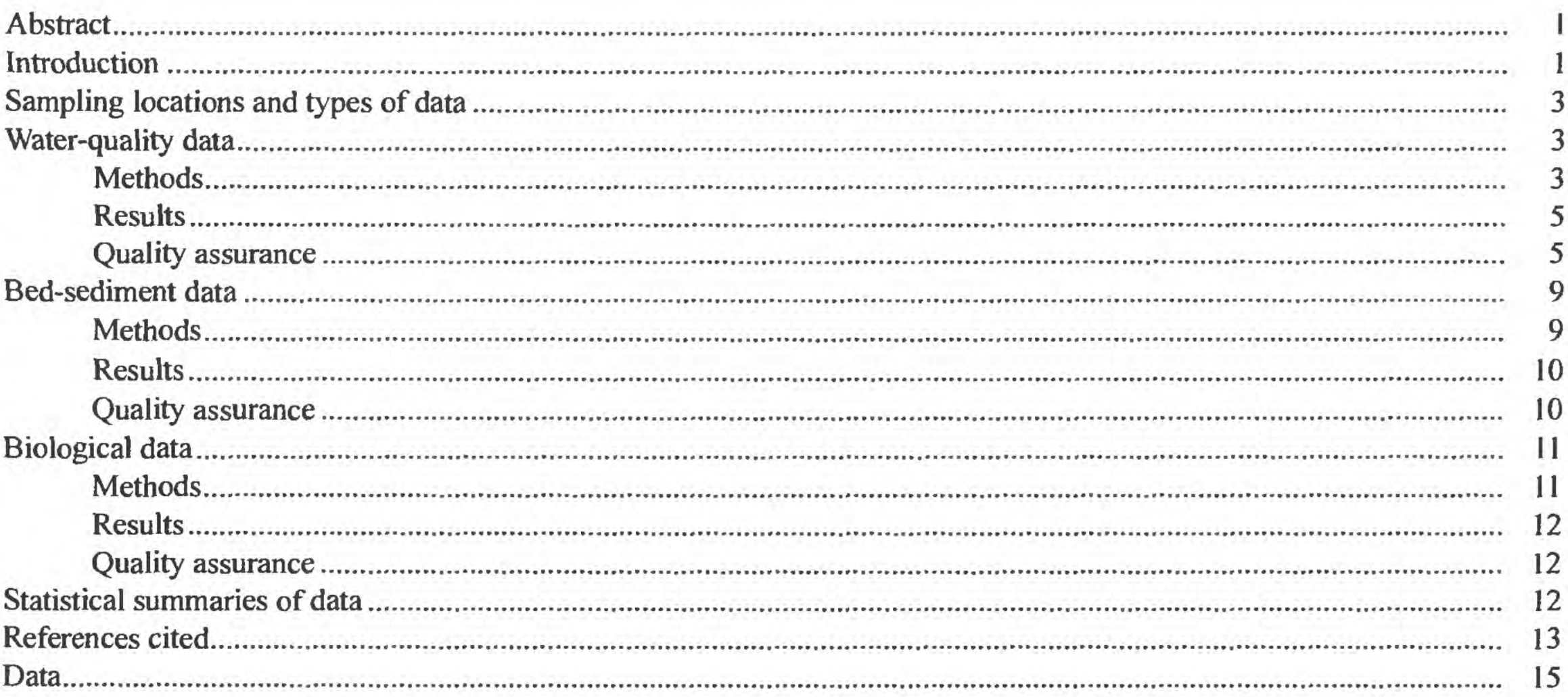

\section{ILLUSTRATION}

Figure 1. Map showing location of study area..

\section{TABLES}

Table 1. Type and period of data collection at sampling stations in the upper Clark Fork basin, Montana

2. Properties measured onsite and constituents analyzed in samples of water, bed sediment, and biota from the upper Clark Fork basin, Montana.

3. Data-quality objectives for analyses of water-quality samples collected in the upper Clark Fork basin, Montana

4. Water-quality data for the upper Clark Fork basin, Montana, October 1998 through September 1999

5-7. Daily streamflow and suspended-sediment data, October 1998 through September 1999, for:

5. Clark Fork at Deer Lodge, Montana

6. Clark Fork at Turah Bridge, near Bonner, Montana .....................................................................

7. Clark Fork above Missoula, Montana

8. Chemical and suspended-sediment analyses of field replicates for water samples, upper Clark Fork basin, Montana

9. Precision of chemical and suspended-sediment analyses of field replicates for water samples, upper Clark Fork basin, Montana

10. Precision of chemical analyses of laboratory replicates for water samples, upper Clark Fork basin, Montana

11. Recovery efficiency for trace-element analyses of laboratory-spiked deionized-water blanks

12. Recovery efficiency for trace-element analyses of laboratory-spiked stream samples, upper Clark Fork basin, Montana

13. Chemical analyses of field blanks for water samples

14. Trace-element analyses of fine-grained bed sediment, upper Clark Fork basin, Montana, August 1999...

15. Trace-element analyses of bulk bed sediment, upper Clark Fork basin, Montana, August 1999 


\section{TABLES (Continued)}

Table 16. Recovery efficiency for tace-enter for bed sediment...

17. Trace-element analyses of procedural blanks for bed sediment........................................................... 56

18. Trace-element analyses of biota, upper Clark Fork basin, Montana, August 1999.................................. 57

19. Recovery efficiency for trace-element analyses of standard reference material for biota ........................... 59

20. Trace-element analyses of procedural blanks for biota ...................................................................... 60

21. Statistical summary of water-quality data for the upper Clark Fork basin, Montana, March 1985 through September 1999

22. Statistical summary of fine-grained bed-sediment data for the upper Clark Fork basin, Montana, August 1986 through August 1999.

23. Statistical summary of bulk bed-sediment data for the upper Clark Fork basin, Montana, August 1993 through August 1999

24. Statistical summary of biological data for the upper Clark Fork basin, Montana, August 1986 through August 1999

\section{CONVERSION FACTORS, ABBREVIATED WATER-QUALITY UNITS, AND ACRONYMS}

\begin{tabular}{rll}
\hline Multiply & By & \multicolumn{1}{c}{ To obtain } \\
\hline cubic foot per second $\left(\mathrm{ft}^{3} / \mathrm{s}\right)$ & & \\
foot $(\mathrm{ft})$ & 0.028317 & cubic meter per second \\
gallon $(\mathrm{gal})$ & 0.3048 & meter $(\mathrm{m})$ \\
gallon $(\mathrm{gal})$ & 3.785 & liter $(\mathrm{L})$ \\
inch (in.) & 3,785 & milliliter $(\mathrm{mL})$ \\
inch (in.) & 25.4 & millimeter $(\mathrm{mm})$ \\
mile (mi) & 25,400 & micrometer $(\mu \mathrm{m})$ \\
ounce (oz) & 1.609 & kilometer \\
part per million & 28.35 & gram $(\mathrm{g})$ \\
square mile (mi $\left.{ }^{2}\right)$ & 1 & microgram per gram $(\mu \mathrm{g} / \mathrm{g})$ \\
ton per day (ton/d) & 2.59 & square kilometer \\
& 907.2 & kilogram per day \\
\hline
\end{tabular}

Temperature can be converted from degrees Celsius $\left({ }^{\circ} \mathrm{C}\right)$ to degrees Fahrenheit $\left({ }^{\circ} \mathrm{F}\right)$ by the equation:

$$
{ }^{\circ} \mathrm{F}=9 / 5\left({ }^{\circ} \mathrm{C}\right)+32
$$

Abbreviated water-quality units used in this report:

$\mu \mathrm{g} / \mathrm{g} \quad$ micrograms per gram

$\mu \mathrm{g} / \mathrm{L} \quad$ micrograms per liter

$\mu \mathrm{g} / \mathrm{mL} \quad$ micrograms per milliliter

$\mu \mathrm{S} / \mathrm{cm} \quad$ microsiemens per centimeter at 25 degrees Celsius

$\mathrm{mg} / \mathrm{L} \quad$ milligrams per liter

Water-year definition:

A water year is the 12-month period from October 1 through September 30. It is designated by the calendar year in which it ends.

Acronyms used in the report:

ICAPES Inductively Coupled Argon Plasma Emission Spectroscopy

LRL laboratory reporting levels

LT-MDL long-term method detection levels

NWQL USGS National Water Quality Laboratory, Denver, Colo.

RSD relative standard deviation

SRM standard reference material

USGS U.S. Geological Survey 


\title{
Water-Quality, Bed-Sediment, and Biological Data (October 1998 through September 1999) and Statistical Summaries of Data for Streams in the Upper Clark Fork Basin, Montana
}

\author{
By Kent A. Dodge, Michelle I. Hornberger ${ }^{1}$, and Carlos Primo C. David ${ }^{1}$
}

Abstract

Water, bed sediment, and biota were sampled in streams from Butte to below Missoula as part of a program to characterize aquatic resources in the upper Clark Fork basin of western Montana. Sampling stations were located on the Clark Fork and major tributaries. Water-quality data were obtained periodically at 15 stations during October 1998 through September 1999 (water year 1999). Data for 16 bed-sediment and 15 biological stations were obtained in August 1999. The primary constituents analyzed were trace elements associated with tailings from historical mining and smelting activities.

Water-quality data include concentrations of selected major ions, trace elements, and suspended sediment in stream samples. Daily values of streamflow, suspended-sediment concentration, and suspendedsediment discharge are given for three stations. Bedsediment data include trace-element concentrations in the fine-grained and bulk fractions. Biological data include trace-element concentrations in whole-body tissue of aquatic benthic insects. Quality-assurance data are reported for analytical results of water, bed sediment, and biota. Statistical summaries of waterquality, bed-sediment, and biological data are provided for the period of record at each station since 1985 .

\section{INTRODUCTION}

The Clark Fork originates near Warm Springs in western Montana at the confluence of Silver Bow and Warm Springs Creeks (fig. 1). Along the 148-mi reach of stream from Silver Bow Creek in Butte to the Clark Fork at Milltown Reservoir, six major tributaries enter: Blacktail Creek, Warm Springs Creek, Little Blackfoot River, Flint Creek, Rock Creek, and Blackfoot River. Principal surface-water uses in the 6,000- $\mathrm{mi}^{2}$ Clark Fork basin above Missoula include irrigation, stock watering, light industry, hydroelectric power generation, and habitat for trout fisheries. Current land uses primarily are cattle production, logging, mining, and recreation. Large-scale mining and smelting had been prevalent land uses in the upper basin for more than one hundred years, but are now largely discontinued.

Deposits of copper, gold, silver, and lead ores were extensively mined, milled, and smelted in the drainages of Silver Bow and Warm Springs Creeks from about 1870 to 1980 . Moderate- and small-scale mining also occurred in the basins of most of the major tributaries to the upper Clark Fork. Tailings derived from mineral processing commonly contain large quantities of trace elements such as arsenic, cadmium, copper, lead, and zinc. Tailings have been eroded, mixed with stream sediment, and transported downstream since the late 1800's and redeposited in stream channels, on flood plains, and in the Warm Springs Ponds and Milltown Reservoir. The widely dispersed tailings continue to be eroded, transported, and redeposited along the stream channel and flood plain, especially during high flows. The occurrence of trace elements in elevated concentrations can pose a risk to aquatic biota and human health because they can accumulate to potentially toxic levels.

Concern about the potential toxicity of tailings to aquatic biota and human health has resulted in a comprehensive effort by State, Federal, and private entities to characterize the aquatic resources in the upper Clark Fork basin to guide and monitor remedial cleanup activities. A long-term data base was considered necessary to detect trends over time in order to evaluate the effectiveness of remediation. Water-quality data have been collected by the U.S. Geological Survey (USGS) at selected sites in the upper Clark Fork basin since 1985 (Lambing, 1987, 1988, 1989, 1990, and 1991; Lambing and others, 1994, 1995; and Dodge and others, 1996, 1997, 1998, 1999). Trace-element data for bed sediment and biota (aquatic benthic insects) have

${ }^{1}$ U.S. Geological Survey, Menlo Park, Calif. 


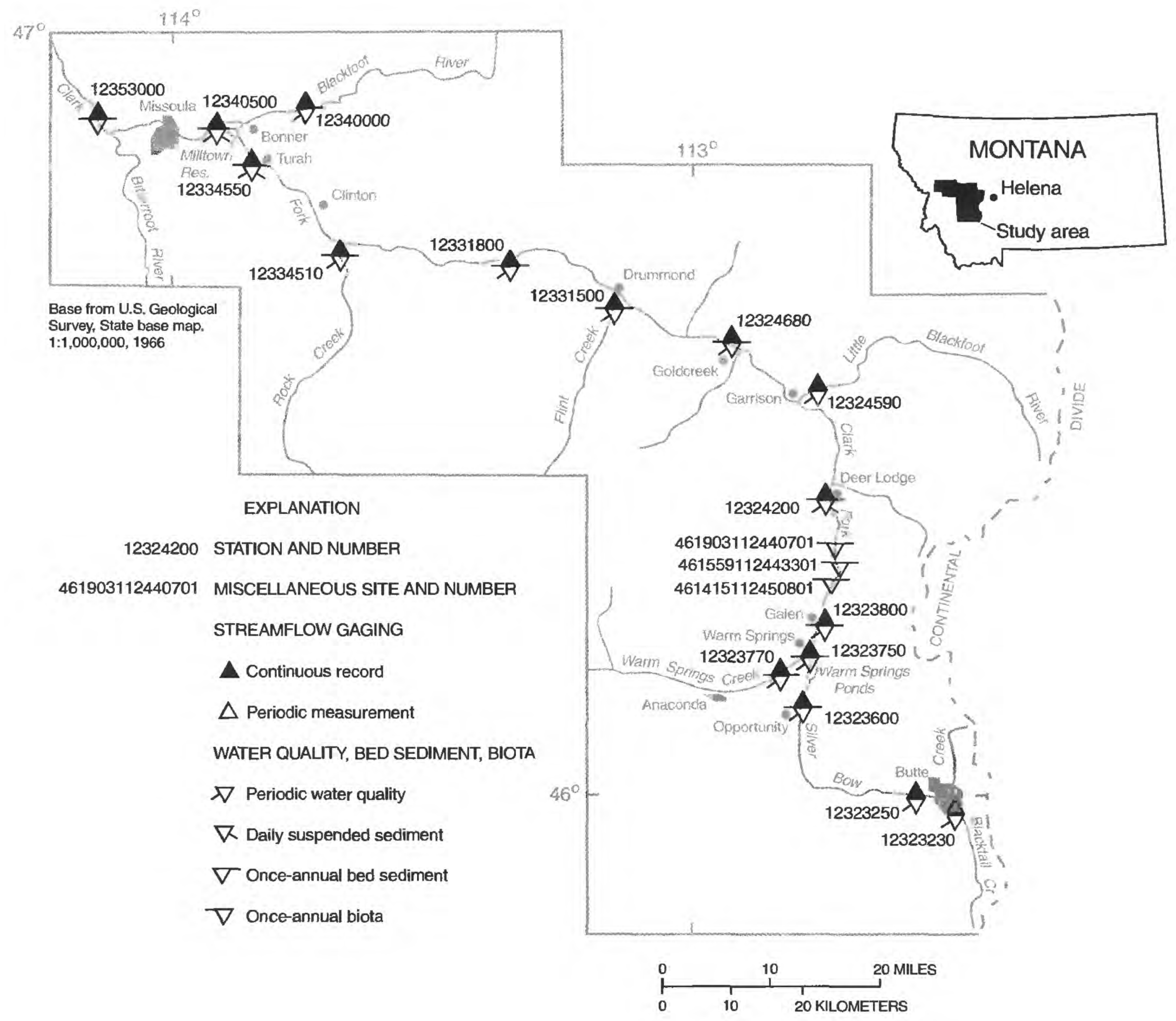

Figure 1. Location of study area.

been collected intermittently since 1986 at selected sites as part of studies on bed-sediment contamination and bioaccumulation of metals conducted by the USGS National Research Program (Axtmann and Luoma, 1991; Axtmann and others, 1997; Cain and others, 1992, 1995; Hornberger and others, 1997). In March 1993, an expanded sampling program for water, bed sediment, and biota was implemented in cooperation with the U.S. Environmental Protection Agency to provide systematic, long-term monitoring. In 1996, water-quality and daily sediment sampling in the expanded program was scaled back to a less extensive network and reduced sampling frequency. In 1997, the water-quality network was partially restored to the pre1996 status (water-quality sampling at the Clark Fork below Missoula was not reactivated) and sampling frequency was increased to better quantify the seasonal and annual variability in selected constituents.

The purpose of this report is to present waterquality data for 15 stations and trace-element data for 16 bed-sediment and 15 biological stations in the upper Clark Fork basin collected from October 1998 through September 1999 (water year 1999). Quality-assurance data are presented for water quality, bed sediment, and biota. Statistical summaries also are provided for

2 Water-quality, bed-sediment, and biological data (October 1998 through September 1999) and statistical summaries of data for streams in the upper Clark Fork basin. Montana 
water-quality, bed-sediment, and biological data collected since 1985.

\section{SAMPLING LOCATIONS AND TYPES OF DATA}

Sampling stations in the upper Clark Fork basin are located on the Clark Fork mainstem and major tributaries from Butte to below Missoula (fig. 1). Mainstem sampling sites were selected to divide the upper Clark Fork into reaches of relatively uniform length, with each reach encompassing either a major tributary or depositional environment (Warm Springs Ponds and Milltown Reservoir). Tributaries were sampled to describe water-quality characteristics for major hydrologic sources in the upper basin and to provide reference comparisons to the mainstem for bed sediment and biota. Water-quality data were obtained periodically at 15 stations; daily suspended-sediment data were obtained at 3 of these stations. Trace-element data for 16 bed-sediment and 15 biological stations were obtained once-annually (table 1).

A list of properties and constituents analyzed in samples of water, bed sediment, and biota is given in table 2. Results of analyses for water, bed sediment, biota, and associated quality assurance for water year 1999 are listed in tables 4 through 20 at the back of the report. Statistical summaries of water-quality, bed-sediment, and biological data collected between March 1985 and September 1999 are given in tables 21-24 at the back of the report.

Quality assurance of data was maintained through the use of documented procedures designed to provide environmentally representative data. Acceptable performance of the procedures was verified with quality-control samples that were collected systematically to provide a measure of the accuracy, precision, and bias of the environmental data and to identify problems associated with sampling, processing, or analysis.

\section{WATER-QUALITY DATA}

Water-quality data consist of measurements of physical properties and concentrations of chemical and physical constituents analyzed in stream samples. Samples were collected 6 to 8 times per year on a schedule designed to describe seasonal and hydrologic variability. In addition, supplemental samples were collected by a contract observer at Clark Fork at Turah
Bridge, near Bonner (11 samples), Blackfoot River near Bonner (4 samples), and Clark Fork above Missoula ( 13 samples) to better define changes in trace-element concentrations and transport during extended high flows, and during the lowering of Milltown Reservoir water levels for dam maintenance.

\section{Methods}

Cross-sectional water samples were collected from multiple verticals across the stream using depthand width-integration methods described by USGS (1977), Knapton (1985), and Edwards and Glysson (1999). These methods provide a vertically and laterally discharge-weighted sample that is representative of the entire flow through the cross section of a stream. Sampling equipment consisted of standard USGS depth-integrating suspended-sediment samplers (DH48, DH-81, and D-74TM), which are either constructed of plastic or coated with a non-metallic epoxy paint, and equipped with nylon nozzles.

Onsite measurements of water temperature, specific conductance, and $\mathrm{pH}$ were made during collection of periodic water-quality samples. Onsite sample processing, including filtration and preservation, was performed according to procedures described by Horowitz and others (1994), Ward and Harr (1990), and Knapton (1985). Instantaneous streamflow at the time of water sampling was determined at all stations, either by direct measurement or from stage-discharge rating tables (Rantz and others, 1982).

Water samples were analyzed for the constituents listed in table 2 by the USGS National Water Quality Laboratory (NWQL) in Denver, Colo. The trace elements arsenic, cadmium, copper, iron, lead, manganese, and zinc were analyzed for both dissolved and total-recoverable concentrations. Analytical methods are described by Fishman and Friedman (1989) and Fishman (1993).

Cross-sectional water samples also were collected for analysis of suspended sediment whenever periodic water-quality samples were collected. These samples were analyzed for suspended-sediment concentration and the percentage of suspended sediment finer than $0.062-\mathrm{mm}$ diameter (silt size and smaller) by the USGS sediment laboratory in Helena, Mont., according to methods described by Guy (1969) and Lambing and Dodge (1993). 


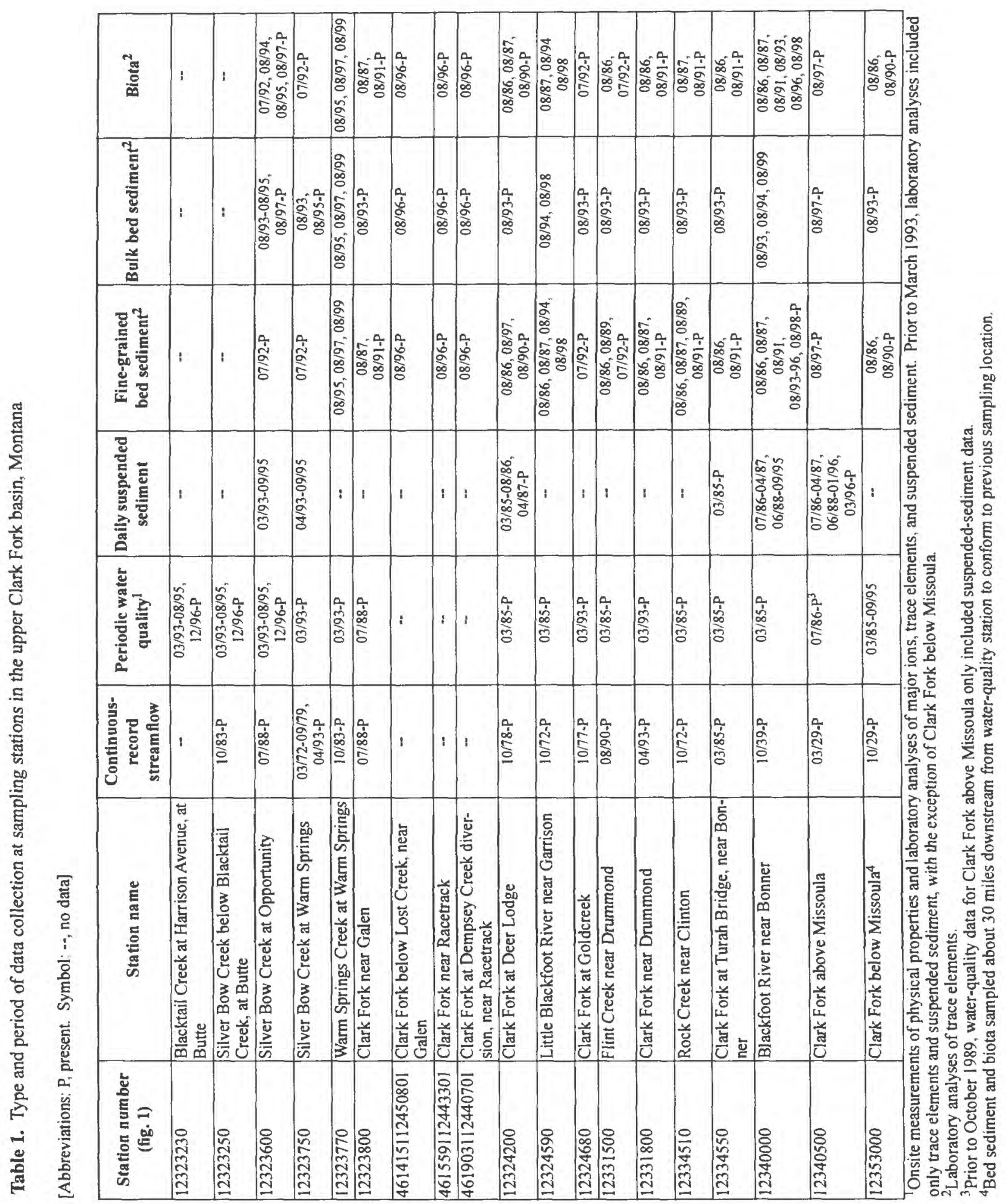

4 Water-quality, bed-sediment, and biological data (October 1998 through September 1999) and statistical summaries of data for streams in the upper Clark Fork basin, Montana 
Table 2. Properties measured onsite and constituents analyzed in samples of water, bed sediment, and biota from the upper Clark Fork basin, Montana

\begin{tabular}{|c|c|c|c|}
\hline \multicolumn{2}{|c|}{ Water } & \multirow{2}{*}{$\frac{\text { Bed sediment }}{\text { Constituent }}$} & \multirow{2}{*}{$\frac{\text { Biota }}{\text { Constituent }}$} \\
\hline Property & Constituent & & \\
\hline Streamflow & Hardness & Cadmium & Cadmium \\
\hline Specific conductance & Calcium & Chromium & Chromium \\
\hline $\mathrm{pH}$ & Magnesium & Copper & Copper \\
\hline \multirow[t]{8}{*}{ Temperature } & Arsenic & Iron & Iron \\
\hline & Cadmium & Lead & Lead \\
\hline & Copper & Manganese & Manganese \\
\hline & Iron & Nickel & Nickel \\
\hline & Lead & Silver & Zinc \\
\hline & Manganese & Zinc & \\
\hline & Zinc & & \\
\hline & Suspended sediment & & \\
\hline
\end{tabular}

At the three daily suspended-sediment stations (table 1), suspended-sediment samples were collected 2 to 8 times per week. These samples were collected by local contract observers using the depth-integration method at a single vertical near mid-stream. The samples were analyzed for suspended-sediment concentration and were used to determine daily mean suspended-sediment concentrations according to methods described by Porterfield (1972).

\section{Results}

Water-quality data for samples collected periodically during October 1998 through September 1999 (water year 1999) are presented in table 4. The types of data include instantaneous streamflow, onsite measurements of water-quality properties, and analytical results for chemical constituents and suspended sediment.

Daily streamflow and suspended-sediment data for water year 1999 at the three daily suspended-sediment stations are given in tables 5 through 7 . Monthly descriptive statistics for each parameter are provided along with totals for the annual discharge of water and suspended sediment.

\section{Quality Assurance}

Quality-assurance procedures used for the collection and field processing of water-quality samples are described by Horowitz and others (1994), Ward and Harr (1990), Edwards and Glysson (1999), Knapton and Nimick (1991), and Knapton (1985). Standard procedures used by the NWQL for internal sample handling and quality assurance are described by Friedman and Erdmann (1982), Jones (1987), and Pritt and Raese (1992). Quality-assurance procedures used by the Montana District sediment laboratory are described by Lambing and Dodge (1993).

The quality of analytical results reported for water-quality samples was evaluated by quality-control samples that were submitted from the field and analyzed concurrently in the laboratory with routine samples. These quality-control samples consisted of replicates, spikes, and blanks which provide quantitative information on the precision and bias of the overall field and laboratory process. Each type of quality-control sample was submitted at a proportion equivalent to about 5 percent of the total number of water-quality samples. Therefore, the total number of quality-control samples represented about 15 percent of the total number of water-quality samples.

In addition to quality-control samples submitted from the field, internal quality-assurance practices at the NWQL are performed systematically to provide quality control of analytical procedures (Pritt and Raese, 1992). These internal practices include analyses of quality-control samples such as calibration standards, standard reference water samples, replicate samples, deionized-water blanks, or spiked samples at 
a proportion equivalent to at least 10 percent of the sample load. The NWQL participates in a blindsample program where standard reference water samples prepared by the USGS Branch of Quality Systems are routinely inserted into the sample line for each analytical method at a frequency proportional to the sample load. The laboratory also participates in external evaluation studies twice-yearly with the U.S. Environmental Protection Agency, the Canadian Center for Inland Water, and the Branch of Quality Systems to assess analytical performance.

Replicate data can be obtained in different ways to provide an assessment of precision (reproducibility) of analytical results. Replicate samples are two or more samples considered to be essentially identical in composition. Replicate samples can be obtained in the field by either repeating the collection process to obtain two or more samples or by splitting a single sample into two or more subsamples which are then analyzed separately (field replicate). Likewise, a single sample can be analyzed two or more times in the laboratory to obtain a measure of analytical variability (laboratory replicate).

Precision of analytical results for field replicates is affected by numerous sources of variability within the field and laboratory environments, including sample collection, sample processing, and sample analysis. To provide data on precision for samples exposed to all sources of variability, chemical-replicate samples were obtained in the field by splitting a composite stream sample. Suspended-sediment replicate samples were obtained in the field by concurrently collecting two independent cross-sectional samples. Analyses of these field replicates indicate the reproducibility of environmental data that are affected by the combined variability potentially introduced by field and laboratory processes.

Analytical precision was evaluated with laboratory replicates, which excluded field sources of variability. Two independent analyses were made of an individual sample selected randomly in the laboratory from the group of samples comprising each analytical run. A separate analysis of the sample was made at the beginning and end of each analytical run to provide information on the reproducibility of laboratory analytical results independent of possible variability caused by field collection and processing of samples.

Spiked samples are used to evaluate the ability of an analytical method to accurately measure a known amount of analyte added to a sample. Because some constituents in stream water can potentially interfere with the analysis of a targeted analyte, it is important to determine whether such effects are causing inaccurate analyses. Deionized-water blanks and aliquots of stream samples were spiked in the laboratory with known amounts of the same trace elements analyzed in water samples. Analyses of spiked blanks indicate if the spiking procedure and analytical method are within control for a water matrix that is presumably free of chemical interference. Analyses of spiked aliquots of stream samples indicate if the chemical matrix of the stream water interferes with the analytical measurement and whether these interferences could contribute significant bias to reported trace-element concentrations for stream samples.

Blank samples of deionized water were routinely analyzed to identify the presence and magnitude of contamination that potentially could bias analytical results. The particular type of blank sample routinely tested was a "field" blank. Field blanks are aliquots of deionized water that are certified as trace-element free and are processed through the sampling equipment used to collect stream samples. These blanks are then subjected to the same processing (sample splitting, filtration, preservation, transportation, and laboratory handling) as stream samples. Blank samples are analyzed for the same constituents as those of stream samples to identify whether any detectable concentrations exist.

All water samples were handled in accordance with chain-of-custody procedures that provide documentation of sample identity, shipment, receipt, and laboratory handling. All samples submitted from a sampling episode were stored and analyzed as a discrete sample group, independent of other samples submitted to the NWQL. Therefore, statistical descriptions of quality-control data generated for this program are directly applicable to the analytical results for stream samples reported herein.

Data-quality objectives (table 3 ) were established for water-quality data as part of the study plan for the expanded long-term monitoring program that was initiated in 1993. The objectives identify analytical requirements of detectability and serve as a guide for identifying questionable data by establishing acceptable limits for precision and bias of laboratory results. Comparisons of quality-control data to dataquality objectives are used to evaluate whether sampling and analytical procedures are producing environmentally representative data in a consistent manner. 
Data that did not meet the objectives were evaluated for acceptability, and corrective action was taken, when appropriate.

Changes in analytical methods during the year resulted in the modification of the minimum reporting levels for total-recoverable cadmium, total-recoverable iron, total-recoverable and dissolved manganese, and total-recoverable and dissolved zinc. Because these changes occurred part way through the year, the minimum reporting levels for these constituents are represented as a range of detectability in table 3 . The minimum reporting levels for dissolved magnesium, dissolved iron, and dissolved lead also changed, but near the beginning of the year; therefore, these minimum reporting levels remained constant throughout the year.

During water year 1999, the NWQL began implementation of a new, statistically based convention for establishing reporting levels and for reporting lowconcentration data (Childress and others, 1999). Quality-control data are collected on a continuing basis to determine long-term method detection levels (LT-
MDLs) and laboratory reporting levels (LRLs). These values are re-evaluated each year and, consequently, may change from year to year. The methods are designed to limit the possible occurrence of a false positive or false negative error to 1 percent or less. Accordingly, concentrations are reported as $<\mathrm{LRL}$ for samples in which the analyte was either not detected or did not pass identification criteria. Analytes that are detected at concentrations between the LT-MDL and LRL and that pass identification criteria are estimated. Estimated concentrations are noted with a remark code of "E." These data need to be used with the understanding that their uncertainty is greater than that of data reported without the " $E$ " remark code.

The precision of analytical results for a constituent can be determined by estimating a standard deviation of the differences between replicate measurements for several sets of samples. These replicate measurements may consist either of individual analyses of a pair of samples considered to be essentially identical (field replicates) or multiple analyses of an individual sample (laboratory replicates). The dif-

Table 3. Data-quality objectives for analyses of water-quality samples collected in the upper Clark Fork basin, Montana

[Abbreviations: $\mu \mathrm{g} / \mathrm{L}$, micrograms per liter; $\mathrm{mg} / \mathrm{L}$, milligrams per liter; $\mathrm{mm}$, millimeter. Symbol: -- , not determined]

\begin{tabular}{|c|c|c|c|c|}
\hline \multirow[b]{3}{*}{ Constituent } & \multicolumn{4}{|c|}{ Data-quality objectives } \\
\hline & \multicolumn{2}{|c|}{ Detectability } & \multirow{2}{*}{$\begin{array}{c}\text { Precision } \\
\text { Maximum relative } \\
\text { standard deviation of } \\
\text { laboratory replicate } \\
\text { analyses, } \\
\text { in percent }\end{array}$} & \multirow{2}{*}{$\begin{array}{c}\text { Bias } \\
\begin{array}{c}\text { Maximum } \\
\text { deviation of } \\
\text { spike recovery, } \\
\text { in percent }\end{array}\end{array}$} \\
\hline & $\begin{array}{r}\text { Labo } \\
\text { repo } \\
\text { le }\end{array}$ & $\begin{array}{l}\text { ratory } \\
\text { orting } \\
\text { vel }\end{array}$ & & \\
\hline Calcium, dissolved & 0.02 & $\mathrm{mg} / \mathrm{L}$ & 20 & -- \\
\hline Magnesium, dissolved & .004 & $\mathrm{mg} / \mathrm{L}$ & 20 & - \\
\hline Arsenic, total recoverable & 1 & $\mu \mathrm{g} / \mathrm{L}$ & 20 & 25 \\
\hline Arsenic, dissolved & 1 & $\mu \mathrm{g} / \mathrm{L}$ & 20 & 25 \\
\hline Cadmium, total recoverable & $.1-1$ & $\mu \mathrm{g} / \mathrm{L}$ & 20 & 25 \\
\hline Cadmium, dissolved & .1 & $\mu \mathrm{g} / \mathrm{L}$ & 20 & 25 \\
\hline Copper, total recoverable & 1 & $\mu \mathrm{g} / \mathrm{L}$ & 20 & 25 \\
\hline Copper, dissolved & 1 & $\mu \mathrm{g} / \mathrm{L}$ & 20 & 25 \\
\hline Iron, total recoverable & $10-14$ & $\mu \mathrm{g} / \mathrm{L}$ & 20 & 25 \\
\hline Iron, dissolved & 10 & $\mu \mathrm{g} / \mathrm{L}$ & 20 & 25 \\
\hline Lead, total recoverable & 1 & $\mu \mathrm{g} / \mathrm{L}$ & 20 & 25 \\
\hline Lead, dissolved & .5 & $\mu \mathrm{g} / \mathrm{L}$ & 20 & 25 \\
\hline Manganese, total recoverable & $3-10$ & $\mu \mathrm{g} / \mathrm{L}$ & 20 & 25 \\
\hline Manganese, dissolved & 1-4 & $\mu \mathrm{g} / \mathrm{L}$ & 20 & 25 \\
\hline Zinc, total recoverable & $10-40$ & $\mu \mathrm{g} / \mathrm{L}$ & 20 & 25 \\
\hline Zinc, dissolved & $1-20$ & $\mu \mathrm{g} / \mathrm{L}$ & 20 & 25 \\
\hline Sediment, suspended & 1 & $\mathrm{mg} / \mathrm{L}$ & -- & -- \\
\hline Sediment, suspended (percent finer than $0.062 \mathrm{~mm}$ ) & 1 & percent & - & - \\
\hline
\end{tabular}


ferences in concentration between replicate analyses can be used to estimate a standard deviation according to the following equation (Taylor, 1987):

$$
S=\sqrt{\frac{\sum d^{2}}{2 k}}
$$

where

$S=$ standard deviation of the difference in concentration between replicate analyses,

$d=$ difference in concentration between each pair of replicate analyses, and

$k=$ number of pairs of replicate analyses.

Precision also can be expressed as a relative standard deviation (RSD), in percent, which is computed from the standard deviation and the mean concentration for all the replicate analyses. Expressing precision relative to a mean concentration standardizes comparison of precision among individual constituents. The RSD, in percent, is calculated according to the following equation (Taylor, 1987):

$$
R S D=\frac{S}{\bar{x}} \times 100
$$

where

$$
\begin{aligned}
& R S D=\text { relative standard deviation, } \\
& S=\text { standard deviation, and } \\
& \bar{x} \quad=\text { mean of all replicate concentrations. }
\end{aligned}
$$

Paired analyses of field replicates are presented in table 8. The precision estimated for each constituent based on these paired results, which include both field and laboratory sources of variability, is reported in table 9. Statistics for precision of field-replicate analyses were based on the values reported in table 8, which are rounded to standard USGS reporting levels for the particular constituent and its analytical method (Timme, 1994).

Data-quality objectives for precision are not directly applicable to field replicates because of the inability to determine whether the variability results from field sample collection and processing, or laboratory handling and analysis. However, a statistical calculation of precision for the field replicates is provided in table 9 to illustrate overall reproducibility of environmental data that incorporates both field and laboratory sources of variability. Relative standard deviations estimated from differences in analytical results between field replicates were within 12 percent for all constituents.
Analytical precision for chemical constituents based on replicate laboratory analyses of individual samples, which includes only laboratory sources of variability, is reported in table 10 . Statistics for analytical precision of laboratory-replicate analyses are based on unrounded values stored in laboratory data files.

The data-quality objective for analytical precision of laboratory-replicate analyses is a maximum relative standard deviation of 20 percent. Precision estimates for laboratory-replicate analyses (table 10) were within the 20-percent relative standard deviation limit for all constituents except dissolved zinc. The exceedance of dissolved zinc was a result of poor comparison in 3 of the 10 laboratory replicate sample sets. The poor precision data for dissolved zinc was random and showed no systematic patterns.

Analyses of an unspiked sample and a spiked aliquot of the same sample provide a measure of the recovery efficiency for the analytical method within the chemical matrix of the sample. Spike recovery, in percent, was calculated using equation 3 (see below).

The data-quality objective for acceptable spike recovery of trace elements in water samples was a maximum deviation of 25 percent from a theoretical 100percent recovery of added constituent. At the laboratory, a spiked deionized-water blank and a spiked aliquot of a stream sample were prepared and analyzed along with the original unspiked sample. The differences between the spiked and unspiked sample concentrations were determined and used to compute recovery according to equation 3 . If the spike recovery for a trace element was outside a range of 75 to 125 percent, the instrument was recalibrated and the entire sample set and spiked samples were reanalyzed for that particular trace element until recoveries were improved to the extent possible. Results of recovery efficiency for individual trace elements in spiked deionized-water blanks and spiked stream samples are presented in tables 11 and 12 , respectively.

The mean spike recovery for deionized-water samples spiked with trace elements ranged from 93.3 to 108.9 percent. The mean spike recovery for spiked stream samples ranged from 96.2 to 105.2 percent. The 95-percent confidence intervals (Taylor, 1987) for the mean of spike recovery for each constituent analyzed in stream samples (table 12) did not exceed a 25-percent deviation from an expected 100-percent recovery. 
Spike recovery, in percent $=\frac{\text { spiked sample concentration }- \text { unspiked sample concentration }}{\text { spike concentration }} \times 100$

High or low bias is indicated if the confidence interval does not include 100 percent recovery. All laboratory-spiked stream samples (table 12) had confidence intervals for percent recovery that included 100 percent. Because the mean spike recoveries met dataquality objectives, no adjustments were made to analytical results for stream samples on the basis of spike recoveries.

Analytical results for field blanks are presented in table 13. A field blank with constituent concentrations equal to or less than the laboratory reporting level (LRL) for the analytical method indicates that the entire process of sample collection, field processing, and laboratory analysis is presumably free of significant contamination. If detectable concentrations in field blanks were equal to or greater than twice the LRL (typical measurement precision at the detection level), the concentrations were noted during data review.

Analytical results from the field blank for the next sample set is evaluated for a consistent trend that may indicate systematic contamination. Sporadic, infrequent exceedances of twice the LRL probably represent random contamination or instrument calibration error that is not persistent in the process and which is not likely to cause significant positive bias in a long-term record of analytical results. However, if concentrations for a particular constituent exceed twice the LRL in field blanks from two consecutive field trips, blank samples are collected from individual components of the processing sequence and are submitted for analysis in order to identify the source of contamination.

Constituent concentrations in field blanks were almost always less than the LRL. There was only one occurrence of a value exceeding twice the minimum reporting level (iron), and there were no occurrences of detectable concentrations for any trace element in two consecutive blank samples. Therefore, the analytical results for field blanks indicate no systematic contamination that would bias the reported water-quality data for stream samples.

\section{BED-SEDIMENT DATA}

Bed-sediment data consist of analyses of traceelement concentrations in the fine-grained and bulk (fine plus coarse) fractions of the bed-sediment sample.
Bed-sediment samples are collected once-annually during low, stable flow conditions to facilitate data comparisons among years.

\section{Methods}

Bed-sediment samples were collected in August 1999 using protocols described by E.V. Axtmann (U.S. Geological Survey, written commun., 1994). Samples were collected from the surfaces of streambed deposits in low-velocity areas near the edge of the stream using an acid-washed polypropylene scoop. Whenever possible, samples were collected from both sides of the stream. Three composite samples of fine-grained bed sediment and two composite samples of bulk bed sediment were collected at each site.

Individual samples of fine-grained bed sediment were collected by scooping material from the surfaces of three to five randomly selected deposits along pool or low-velocity areas. The three to five individual samples were combined to form a single composite sample. This collection process was repeated three times to obtain three composite samples. Each composite sample was wet-sieved onsite through a $0.064-\mathrm{mm}$ nylonmesh sieve using ambient stream water. The fraction of bed sediment in each composite sample that was finer than $0.064 \mathrm{~mm}$ was transferred to an acid-washed 500$\mathrm{mL}$ polyethylene bottle and transported to the laboratory on ice.

Individual samples of bulk bed sediment also were collected by scooping material from the surfaces of three to five randomly selected deposits. Because the streambed at most sampling locations is predominantly gravel and cobble, deposits were selected where cobbles and gravel could be excluded from the samples. Bulk bed-sediment samples were not sieved and generally were composed of particles smaller than about $10 \mathrm{~mm}$ in diameter. The individual unsieved samples were composited into an acid-washed polyethylene bottle and transported to the laboratory on ice.

Bed-sediment samples were prepared for analysis at the USGS National Research Program laboratory in Menlo Park, Calif. Fine-grained and bulk bed-sediment samples were oven-dried at $60^{\circ} \mathrm{C}$ and ground using an acid-washed ceramic mortar and pestle. 
Duplicate aliquots of approximately $0.6 \mathrm{~g}$ of sediment from each of the three composite fine-grained bed sediment samples were digested using a hot, concentrated nitric acid reflux according to methods described by Luoma and Bryan (1981). Two aliquots were similarly digested from the single composite sample of bulk bed sediment. After a digestion period of up to several weeks, the aliquots were evaporated to dryness on a hot plate. The dry residue was redissolved with $20 \mathrm{~mL}$ of $0.6 \mathrm{~N}$ (normal) hydrochloric acid. The reconstituted aliquots then were filtered through a $0.45-\mu \mathrm{m}$ filter using a syringe and in-line disposable filter cartridge. The filtrate was subsequently diluted to either a 1:5 or 1:10 ratio with $0.6 \mathrm{~N}$ hydrochloric acid. These final solutions were analyzed for cadmium, chromium, copper, iron, lead, manganese, nickel, silver, and zinc using Inductively Coupled Argon Plasma Emission Spectroscopy (ICAPES).

\section{Results}

Concentrations of trace elements measured in samples of fine-grained and bulk bed sediment collected during August 1999 are summarized in tables 14 and 15 , respectively. Liquid-phase concentrations, in $\mu \mathrm{g} / \mathrm{mL}$, that were analyzed in the reconstituted aliquots of digested bed sediment were converted to solid-phase concentrations, in $\mu \mathrm{g} / \mathrm{g}$, using the following equation:

$\mu \mathrm{g} / \mathrm{g}=\frac{\mu \mathrm{g} / \mathrm{mL} \times \text { volume of digested sample, in } \mathrm{mL}}{\text { dry weight of sample, in } \mathrm{g} \times \text { dilution ratio }}(4)$

The reported solid-phase concentrations in table 14 and 15 are the means of all analyses of replicate aliquots from each composite sample collected at the site. Because the conversion from liquid-phase to solid-phase concentration is dependent on both the dilution ratio and the dry weight of the sample, minimum reporting levels for some trace elements may differ among stations and among years.

\section{Quality Assurance}

The protocols for field collection and processing of bed-sediment samples are designed to prevent contamination from metal sources. Non-metallic sampling and processing equipment was acid-washed and rinsed with deionized water prior to the first sample collection. Nylon-mesh sieves were washed in a laboratorygrade detergent and rinsed with deionized water. All equipment was given a final rinse onsite with stream water. Sampling equipment that was reused at each site was rinsed between sites with 10-percent nitric acid, deionized water, and stream water. Separate sieves were used at each site and, therefore, did not require between-site cleaning.

Quality assurance of analytical results for bed sediment included laboratory instrument calibration with standard solutions and analysis of quality-control samples designed to identify the presence and magnitude of bias (E.V. Axtmann, U.S. Geological Survey, written commun., 1994). Quality-control samples consisted of standard reference materials and procedural blanks. Each type of sample was analyzed in a proportion equivalent to about 10 to 20 percent of the total number of bed-sediment samples.

Standard reference materials (SRMs) are commercially prepared materials that have certified concentrations of trace elements. Replicate analyses of SRMs are used to indicate the reproducibility of analytical results and the ability of the method to accurately measure a known quantity of a constituent. Recovery efficiency of trace-element analyses of SRMs for bed sediment is summarized in table 16. Two SRMs consisting of agricultural soils representing low and high concentrations of trace elements were analyzed to test recovery efficiency for a range of concentrations generally similar to those occurring in the upper Clark Fork basin. The digestion process used to analyze bedsediment samples is not a "total" digestion (does not liberate elements associated with crystalline lattices); therefore, 100-percent recovery may not be achieved for elements strongly bound to the sediment. The percent recovery of trace elements in SRMs when using less than a total digestion is useful to indicate which trace elements display strong sediment-binding characteristics and whether analytical recovery is consistent between multiple sets of analyses.

Although data-quality objectives have not been established for bed sediment, percent recoveries are shown in table 16 to illustrate analytical performance. Elements with mean recoveries outside a 25 -percent deviation from complete ( 100 percent) recovery were cadmium and chromium for the low-concentration range (SRM 2709), and chromium for the high-concentration range (SRM 2711). Silver was below the analytical detection limit in the low-range SRM (2709). Chromium recovery was consistently low for both SRM 2709 and 2711 (65.6 and 57.9 percent, respectively). The reason for the lack of measurable recover- 
ies is believed to be the result of analyzing concentrations very close to the detection limit, coupled with signal enhancement resulting from matrix interference. No adjustments were made to trace-element concentrations in bed-sediment samples on the basis of recovery efficiencies.

Procedural blanks for bed-sediment samples consisted of the same reagents used for sample digestion and reconstitution. Concentrated nitric acid used for sample digestion was heated and evaporated to dryness. After evaporation, $0.6 \mathrm{~N}$ hydrochloric acid was added quantitatively to the dry residue to obtain the same dilution ratio as that used in the analysis of bed sediment. Procedural blanks, therefore, represent the same chemical matrix as the reagents used to digest and reconstitute bed-sediment samples. Analytical results for procedural blanks can indicate the presence and magnitude of potential contamination associated with sample handling and analysis in the laboratory environment. Results of trace-element analyses of procedural blanks for bed sediment are in table 17.

Analytical results of procedural blanks are reported as a liquid-phase concentration, in $\mu \mathrm{g} / \mathrm{mL}$, which is equivalent to parts per million. Determination of the significance of a detectable blank concentration is based on the magnitude of the equivalent solid-phase concentration, in $\mu \mathrm{g} / \mathrm{g}$, relative to the ambient concentration of the trace element in bed-sediment samples. Because sample weights of individual aliquots may vary, the relative significance of blank concentrations may differ among samples. If a detectable blank concentration, after conversion to a solid-phase concentration, represents 10 percent or more of the ambient solid-phase concentration, then the blank concentration is subtracted to remove potential contamination bias. Almost all procedural blanks had concentrations less than analytical detection levels. Only copper in one procedural blank sample slightly exceeded the calculated limit of detection. None of the detectable concentrations in blanks were greater than 10 percent of the ambient concentration; therefore, no adjustments were made to trace-element concentrations in bed-sediment samples on the basis of procedural blanks.

\section{BIOLOGICAL DATA}

Biological data consist of analyses of trace-element concentrations in the whole-body tissue of aquatic benthic insects. Insect samples are collected once-annually at the same sites and dates as bed-sedi- ment samples (table 1), allowing for a direct comparison of annual results.

\section{Methods}

Insect samples were collected using protocols described in Hornberger and others (1997). Immature stages of aquatic benthic insects were collected using a large nylon-mesh kick net. A single riffle at each station was sampled repeatedly until an adequate number of individuals was collected to provide sufficient mass for analysis. Targeted taxa for collection were Hydropsyche spp., Brachycentrus spp., and Arctopsyche grandis of the Order Trichoptera (caddisflies), and Claassenia sabulosa of the Order Plecoptera (stoneflies). Samples of each taxon were stored separately, by genus, in acid-washed plastic containers. Samples were frozen with dry ice within 1 hour of collection in a small amount of ambient river water. In previous years (1986-98), benthic insects were depurated for a period of 6-8 hours in an effort to evacuate gut contents. In 1998, a comparison of samples collected using both methods showed no significant difference in metal concentrations in benthic insects, with the exception of copper. Average copper concentrations in depurated samples were 8-25 percent lower than samples frozen within one hour of collection (M.I. Hornberger, unpub. data, 2000). The change in the field protocol minimizes the chance of metal loss through cell membranes and is consistent with methods established by Cain and Luoma (1998).

Insect samples were processed and analyzed at the USGS National Research Program laboratory in Menlo Park, Calif. Insects were thawed and rinsed with ultra-pure deionized water to remove particulate matter, then sorted to their lowest possible taxonomic level. When large numbers of specimens were collected from a station, similar-sized individuals were composited into replicate subsamples. Subsamples were placed in tared scintillation vials and oven-dried at $70^{\circ} \mathrm{C}$. Subsamples were weighed to obtain a final dry weight and digested by reflux using concentrated nitric acid (Cain and others, 1992). After digestion, insect samples were evaporated to dryness on a hot plate. The dry residue was reconstituted in $0.6 \mathrm{~N}$ hydrochloric acid, filtered through a $0.45-\mu \mathrm{m}$ filter, and analyzed undiluted by ICAPES for cadmium, chromium, copper, iron, lead, manganese, nickel, and zinc. 


\section{Results}

Concentrations of trace elements in whole-body tissue of aquatic insects collected during August 1999 are summarized in table 18 . The variability in the number of composite samples among species and among sites reflects differences in insect abundance, with the number of composite samples increasing with the relative abundance of insects. Liquid-phase concentrations analyzed in the reconstituted samples were converted to solid-phase concentrations using equation 4. As with bed sediment, minimum reporting levels may differ among sites as a result of variable sample weights. In general, the smaller the biological sample weight (a function of insect abundance), the higher the minimum reporting level. Therefore, higher minimum reporting levels do not necessarily imply a higher trace-element concentration in tissue.

Two species of Hydropsyche were targeted for collection in this study due to their occurrence at most, but not all, sites: Hydropsyche occidentalis and Hydropsyche cockerelli. Hydropsyche species that could not be positively identified were considered to belong to the morosa group and are categorized as Hydropsyche spp. or Hydropsyche morosa group. Arctopsyche grandis and Claasenia sabulosa also were collected, where available, to represent additional insect taxa that are fairly widely distributed in the upper Clark Fork basin.

\section{Quality Assurance}

The protocols for field collection and processing of biota samples are designed to prevent contamination from metal sources. Non-metallic nets, sampling, and processing equipment were employed in all sample collection. Equipment was acid-washed and rinsed in ultra-pure deionized water prior to the first sample collection. Nets and equipment were thoroughly rinsed in ambient stream water at each new mainstem station. New nets were used for the tributary stations. Biota samples were collected along an increasing concentration gradient to minimize effects from potential stationto-station carryover contamination.

Quality assurance of analytical results for biota samples included laboratory instrument calibration with standard solutions and analyses of quality-control samples designed to identify the presence and magnitude of bias. Quality-control samples consisted of a SRM and procedural blanks. Each type of sample was analyzed in a proportion equivalent to about 10 to 20 percent of the total number of biota samples.

Recovery efficiency for trace-element analyses of the SRM for biota is summarized in table 19. The reference material tested was lobster hepatopancreas. Data-quality objectives have not been established for analytical recovery in biota, but percent recoveries are shown to illustrate analytical performance. Mean recoveries were within 10 percent of complete $(100$ percent) recovery for all trace metals, except chromium. A slightly low bias (confidence interval does not include 100 percent) is indicated for manganese and nickel and a slightly high bias is indicated for cadmium and lead. A higher bias was shown for chromium, which may have resulted because of interelement matrix interference. No adjustments were made to the trace-element concentrations for the insect samples on the basis of recovery efficiency.

Results of trace-element analyses of procedural blanks for biota are in table 20. Procedural blanks for biota consisted of the same reagents used to digest and reconstitute tissue of aquatic insects. The blanks were analyzed undiluted at a proportion of one blank per site. Analytical results for blanks indicated no significant contamination bias. With the exception of iron, all concentrations in the blanks were below detection levels. The detectable concentrations are within the range of instrument variability and are insignificant in relation to the measured concentrations in the insect samples; thus, no adjustments were made to trace-element concentrations in biota.

\section{STATISTICAL SUMMARIES OF DATA}

Statistical summaries of water-quality, bed-sediment, and biological data are provided in tables 21-24 for the period of record at each station since 1986. The summaries include the period of record, number of samples, maximum, minimum, mean, and median of concentrations.

Statistical summaries of water-quality data (table 21) are based on results of cross-sectional samples collected periodically by the USGS during the station's period of record. They do not include supplemental single-vertical samples collected by a contract observer at Clark Fork at Turah Bridge near Bonner, Blackfoot River near Bonner, and Clark Fork above Missoula from 1997 to 1999. Inclusion of supplemental sample results would disproportionately skew the long-term statistics at these three sites relative to the other sites in

12 Water-quality, bed-sediment, and biological data (October 1998 through September 1999) and statistical summaries of data for streams in the upper Clark Fork basin, Montana 
the network. Statistical summaries of bed-sediment (table 22 and 23) and biological data (table 24) are based on results of samples collected once-annually during the indicated years. Because not all stations were sampled for bed sediment and biota every year, these data do not represent a consecutive annual record.

Sample sizes and statistics for bed-sediment data are based on the annual mean concentrations determined from the combined results of composite samples for a given year. Therefore, sample sizes for bed sediment represent the number of years sampled. Sample sizes and statistics for biological data are based on individual analyses for each composite sample collected in individual years rather than the combined annual mean concentration. Biota sample sizes therefore reflect differences in species abundances at each site and among all years. The statistics for biota describe the full range of trace-element concentrations measured among all available composite samples. The abundance of aquatic insects at a particular site in a given year limits the biomass of the sample which, in turn, may result in different taxa analyzed among years or in variable analytical detection limits. Where minimum reporting levels vary among years, statistical summaries are provided only as a general indication of the range of detection.

The presence or absence of insect species at a given site can vary among years and may result in different taxa being analyzed in the long-term period of record. Because Hydropsyche insects were not sorted to the species level during 1986-89, statistics for stations sampled during those years are based on the results of all Hydropsyche species combined. At some sites, statistics for the Hydropsyche morosa group are based on the combined results for two or more species because these samples could not be identified clearly to the species, but had morosa characteristics.

\section{REFERENCES CITED}

Axtmann, E.V., Cain, D.J., and Luoma, S.N., 1997, Effect of tributary inflows on the distribution of trace metals in fine-grained sediment and benthic insects of the Clark Fork River, Montana: Environmental Science and Technology v. 31, p. 750-758.

Axtmann, E.V., and Luoma, S.N., 1991, Large scale distribution of metal contamination in the fine-grained sediment of the Clark Fork River, Montana: Applied Geochemistry, v. 6, p. 75-88.

Cain, D.J., and Luoma, S.H., 1998, Metal exposures to native populations of the caddisfly Hydropsyche
(Trichoptera: Hydropsychidae) determined from cytosolic and whole body metal concentrations: Hydrobiologia, v. 386, p. 103-117.

Cain, D.J., Luoma, S.N., and Axtmann, E.V., 1995, Influence of gut content in immature aquatic insects on assessments of environmental metal contamination: Canadian Joumal of Fisheries and Aquatic Sciences, v. 52 , no. 12 , p. $2736-2746$.

Cain, D.J., Luoma, S.N., Carter, J.L., and Ferd, S.V., 1992, Aquatic insects as bioindicators of trace element contamination in cobble-bottom rivers and streams: Canadian Journal of Fisheries and Aquatic Sciences, v. 49 , no. 10 , p. $2141-2154$.

Childress, C.T., Foreman, W.T., Connor, B.F., and Maloney, T.J., 1999, New reporting procedures based on longterm method detection levels and some considerations for interpretations of water-quality data provided by the U.S. Geological Survey National Water Quality Laboratory: U.S. Geological Survey Open-File Report 99-193, 19 p.

Dodge, K.A., Hornberger, M.I., and Axtmann, E.V., 1996, Water-quality, bed-sediment, and biological data (October 1994 through September 1995) and statistical summaries of data for streams in the upper Clark Fork basin, Montana: U.S. Geological Survey Open-File Report 96-432, 109 p.

1997, Water-quality, bed-sediment, and biological data (October 1995 through September 1996) and statistical summaries of data for streams in the upper Clark Fork basin, Montana: U.S. Geological Survey Open-File Report 97-552, 91 p.

1998, Water-quality, bed-sediment, and biological data (October 1996 through September 1997) and statistical summaries of data for streams in the upper Clark Fork basin, Montana: U.S. Geological Survey Open-File Report 98-407, 102 p.

1999, Water-quality, bed-sediment, and biological data (October 1997 through September 1998) and statistical summaries of data for streams in the upper Clark Fork basin, Montana: U.S. Geological Survey Open-File Report 99-251, $102 \mathrm{p}$.

Edwards, T.K., and Glysson, G.D., 1999, Field methods for measurement of fluvial sediment: U.S. Geological Survey Techniques of Water-Resources Investigations, Book 3, Chapter C2, 89 p.

Fishman, M.J., 1993, Methods of analysis by the U.S. Geological Survey National Water Quality Laboratory--Determination of inorganic and organic constituents in water and fluvial sediments: U.S. Geological Survey Open-File Report 93-125, 217 p.

Fishman, M.J., and Friedman, L.C., eds., 1989, Methods for determination of inorganic substances in water and fluvial sediments: U.S. Geological Survey Techniques of Water-Resources Investigations, book 5, chap. A I, $545 \mathrm{p}$.

Friedman, L.C., and Erdman, D.E., 1982, Quality assurance practices for the chemical and biological analyses of water and fluvial sediments: U.S. Geological Survey 
Techniques of Water-Resources Investigations, book 5, chap. A6, $181 \mathrm{p}$.

Guy, H.P., 1969, Laboratory theory and methods for sediment analysis: U.S. Geological Survey Techniques of Water-Resources Investigations, book 5, chap. Cl, $58 \mathrm{p}$.

Helsel, D.R., and Cohn, T.A., 1988, Estimation of descriptive statistics for multiply censored water quality data: Water Resources Research, v. 24 , no. 12 , p. 1997-2004.

Hornberger, M.I., Lambing, J.H., Luoma, S.N., and Axtmann, E.V., 1997, Spatial and temporal trends of trace metals in surface water, bed sediment, and biota of the upper Clark Fork basin, Montana, 1985-95: U.S. Geological Survey Open-File Report 97-669, 84 p.

Horowitz, A.J., Demas, C.R., Fitzgerald, K.K., Miller, T.L., and Rickert, D.A., 1994, U.S. Geological Survey protocol for the collection and processing of surfacewater samples for the subsequent determination of inorganic constituents in filtered water: U.S. Geological Survey Open-File Report 94-539, 57 p.

Jones, B.E., 1987, Quality control manual of the U.S. Geological Survey's National Water Quality Laboratory: U.S. Geological Survey Open-File Report $87-457,17 \mathrm{p}$.

Knapton, J.R., 1985, Field guidelines for collection, treatment, and analysis of water samples, Montana District: U.S. Geological Survey Open-File Report 85-409, 86 p.

Knapton, J.R., and Nimick, D.A., 1991, Quality assurance for water-quality activities of the U.S. Geological Survey in Montana: U.S. Geological Survey Open-File Report 91-216, $41 \mathrm{p}$.

Lambing, J.H., 1987, Water-quality data for the Clark Fork and selected tributaries from Deer Lodge to Milltown, Montana, March 1985 through June 1986: U.S. Geological Survey Open-File Report 87-110, 48 p. 1988, Water-quality data (July 1986 through September 1987) and statistical summaries (March 1985 through September 1987) for the Clark Fork and selected tributaries from Deer Lodge to Missoula, Montana: U.S. Geological Survey Open-File Report 88-308, 55 p.

1989, Water-quality data (October 1987 through September 1988) and statistical summaries (March 1985 through September 1988) for the Clark Fork and selected tributaries from Galen to Missoula, Montana: U.S. Geological Survey Open-File Report 89-229, 51 p. 1990, Water-quality data (October 1988 through September 1989) and statistical summaries (March 1985 through September 1989) for the Clark Fork and selected tributaries from Galen to Missoula, Montana: U.S. Geological Survey Open-File Report 90-168, 68 p.
1991, Water-quality and transport characteristics of suspended sediment and trace elements in streamflow of the upper Clark Fork basin from Galen to Missoula, Montana, 1985-90: U.S. Geological Survey WaterResources Investigations Report 91-4139, 73 p.

Lambing, J.H., and Dodge, K.A., 1993, Quality assurance for laboratory analysis of suspended-sediment samples by the U.S. Geological Survey in Montana: U.S. Geological Survey Open-File Report 93-131, 34 p.

Lambing, J.H., Hornberger, M.I., Axtmann, E.V., and Dodge, K.A., 1995, Water-quality, bed-sediment, and biological data (October 1993 through September 1994) and statistical summaries of data for streams in the upper Clark Fork basin, Montana: U.S. Geological Survey Open-File Report 95-429, 104 p.

Lambing, J.H., Hornberger, M.I., Axtmann, E.V., and Pope, D.A., 1994, Water-quality, bed-sediment, and biological data (October 1992 through September 1993) and statistical summaries of water-quality data (March 1985 through September 1993) for streams in the upper Clark Fork basin, Montana: U.S. Geological Survey Open-File Report 94-375, 85 p.

Luoma, S.N., and Bryan, G.W., 1981, A statistical assessment of the form of trace metals in oxidized estuarine sediments employing chemical extractants: Science of the Total Environment, v. 17, p. 167-196.

Porterfield, George, 1972, Computation of fluvial-sediment discharge: U.S. Geological Survey Techniques of Water-Resources Investigations, book 3, chap. C3, $66 \mathrm{p}$.

Pritt, J.W., and Raese, J.W., eds., 1992, Quality assurance/quality control manual--National Water Quality Laboratory: U.S. Geological Survey Open-File Report 92-495, 33 p.

Rantz, S.E., and others, 1982, Computation of discharge: U.S. Geological Survey Water-Supply Paper 2175, 2 v., $631 \mathrm{p}$.

Taylor, J.K., 1987, Quality assurance of chemical measurements: Chelsea, Mich., Lewis Publishers, $328 \mathrm{p}$.

Timme, P.J., 1994, National Water Quality Laboratory 1994 Services Catalog: U.S. Geological Survey Open-File Report 94-304, 103 p.

U.S. Geological Survey, 1977, National handbook of recommended methods for water-data acquisition-Chap. 5, Chemical and physical quality of water and sediment: Office of Water Data Coordination, $193 \mathrm{p}$.

Ward, J.R., and Harr, C.A., eds., 1990, Methods for collection and processing of surface-water and bedmaterial samples for physical and chemical analyses: U.S. Geological Survey Open-File Report 90-140, 71 p.

14 Water-quality, bed-sediment, and biological data (October 1998 through September 1999) and statistical summaries of data for streams in the upper Clark Fork basin, Montana 


\section{DATA}


Table 4. Water-quality data for the upper Clark Fork basin, Montana, October 1998 through September 1999

[Abbreviations: $\mathrm{ft}^{3} / \mathrm{s}$, cubic feet per second; ${ }^{\circ} \mathrm{C}$, degrees Celsius; $\mathrm{E}$, estimated; $\mu \mathrm{g} / \mathrm{L}$, micrograms per liter; $\mu \mathrm{S} / \mathrm{cm}$, microsiemens per centimeter at $25^{\circ} \mathrm{C}$; $\mathrm{mg} / \mathrm{L}$. milligrams per liter; mm, millimeter, ton/d, tons per day. Symbols: <, less than laboratory reporting level; --, no data]

12323230-BLACKTAIL CREEK AT HARRISON AVENUE, AT BUTTE, MONT.

\begin{tabular}{|c|c|c|c|c|c|c|c|c|c|}
\hline Date & Time & $\begin{array}{c}\text { Streamflow, } \\
\text { instan- } \\
\text { taneous } \\
\left(\mathrm{ft}^{3} / \mathrm{s}\right)\end{array}$ & $\begin{array}{c}\text { Specific } \\
\text { conductance, } \\
\text { onsite } \\
(u S / c m)\end{array}$ & $\begin{array}{c}\text { pH, } \\
\text { onsite } \\
\text { (standard } \\
\text { units) }\end{array}$ & $\begin{array}{l}\text { Temper- } \\
\text { ature, } \\
\text { water } \\
\left({ }^{\circ} \mathrm{C}\right)\end{array}$ & $\begin{array}{c}\text { Hardness, } \\
\text { total } \\
\text { (mg/L as } \\
\left.\mathrm{CaCO}_{3}\right)\end{array}$ & $\begin{array}{l}\text { Calcium, } \\
\text { dissolved } \\
\text { (mg/L) }\end{array}$ & $\begin{array}{l}\text { Magne- } \\
\text { sium, } \\
\text { dissolved } \\
\text { (mg/L) }\end{array}$ & $\begin{array}{c}\text { Arsenic, } \\
\text { total } \\
\text { recoverable } \\
(\mu \mathrm{g} / \mathrm{L})\end{array}$ \\
\hline \multicolumn{10}{|l|}{ NOV 1998} \\
\hline $17 \ldots$ & 0835 & 8.2 & 286 & 79 & 4.5 & 110 & 32 & 7.8 & 2 \\
\hline \multicolumn{10}{|l|}{ FEB 1999} \\
\hline $22 .$. & 1000 & 6.1 & 294 & 8.2 & 3.0 & 120 & 34 & 8.4 & 2 \\
\hline \multicolumn{10}{|l|}{ APR } \\
\hline $27 \ldots$ & 1020 & 14 & 236 & 8.0 & 7.0 & 93 & 27 & 6.5 & 4 \\
\hline \multicolumn{10}{|l|}{ MAY } \\
\hline $12 \ldots$ & 1425 & 15 & 231 & 8.1 & 9.5 & 89 & 26 & 6.2 & 4 \\
\hline $30 \ldots$ & 1015 & 31 & 185 & 7.7 & 6.5 & 73 & 21 & 5.0 & 8 \\
\hline \multicolumn{10}{|l|}{ JUN } \\
\hline 22. & 0845 & 13 & 237 & 7.8 & 11.5 & 97 & 28 & 6.7 & 7 \\
\hline \multicolumn{10}{|l|}{ AUG } \\
\hline $12 \ldots$ & 1115 & 83 & 283 & 8.1 & 12.5 & 110 & 31 & 7.6 & 5 \\
\hline
\end{tabular}

\begin{tabular}{|c|c|c|c|c|c|c|c|c|}
\hline Date & $\begin{array}{c}\text { Arsenic, } \\
\text { dissolved } \\
(\mu \mathrm{g} / \mathrm{L})\end{array}$ & $\begin{array}{l}\text { Cadmium, } \\
\text { total } \\
\text { recoverable } \\
(\mu \mathrm{g} / \mathrm{L})\end{array}$ & $\begin{array}{l}\text { Cadmium, } \\
\text { dissolved } \\
(\mu \mathrm{g} / \mathrm{L})\end{array}$ & $\begin{array}{c}\text { Copper, } \\
\text { total } \\
\text { recoverable } \\
(\mu \mathrm{g} / \mathrm{L})\end{array}$ & $\begin{array}{l}\text { Copper, } \\
\text { dissolved } \\
(\mu \mathrm{g} / \mathrm{L})\end{array}$ & $\begin{array}{c}\text { Iron, } \\
\text { total } \\
\text { recoverable } \\
(\mu \mathrm{g} / \mathrm{L})\end{array}$ & $\begin{array}{c}\text { Iron, } \\
\text { dissolved } \\
(\mu \mathrm{g} / \mathrm{L})\end{array}$ & $\begin{array}{c}\text { Lead, } \\
\text { total } \\
\text { recoverable } \\
(\mu \mathrm{g} / \mathrm{L})\end{array}$ \\
\hline \multicolumn{9}{|l|}{ NOV 1998} \\
\hline $17 \ldots$ & 2 & $<1$ & $<0.1$ & 2 & 2 & 260 & 100 & $<1$ \\
\hline \multicolumn{9}{|l|}{ FEB 1999} \\
\hline $22 \ldots$ & 2 & $<\mathbf{l}$ & $<.1$ & 2 & I & 250 & 42 & $<1$ \\
\hline \multicolumn{9}{|l|}{ APR } \\
\hline $27 .$. & 2 & $<\mathbf{I}$ & .1 & 8 & 3 & 814 & 200 & 5 \\
\hline \multicolumn{9}{|l|}{ MAY } \\
\hline $12 \ldots$ & 3 & $<1$ & $<.1$ & 5 & 3 & 471 & 180 & $<1$ \\
\hline $30 \ldots$ & 5 & $<1$ & $<.1$ & 14 & 5 & 1,280 & 260 & 3 \\
\hline \multicolumn{9}{|l|}{ JUN } \\
\hline $22 \ldots$ & 6 & $<1$ & $<.1$ & 5 & 4 & 861 & 330 & $<1$ \\
\hline \multicolumn{9}{|l|}{ AUG } \\
\hline $12 \ldots$ & 3 & $<\mathbf{I}$ & $<.1$ & 6 & 3 & 449 & 100 & 1 \\
\hline Date & $\begin{array}{c}\text { Lead, } \\
\text { dissolved } \\
(\mu \mathrm{g} / \mathrm{L})\end{array}$ & $\begin{array}{l}\text { Manga- } \\
\text { nese, } \\
\text { total } \\
\text { recoverable } \\
\text { (ug/L) }\end{array}$ & $\begin{array}{l}\text { Manga- } \\
\text { nese, } \\
\text { dissolved } \\
(\mu \mathrm{g} / \mathrm{L})\end{array}$ & $\begin{array}{c}\text { Zinc, } \\
\text { total } \\
\text { recoverable } \\
(\mu \mathrm{g} / \mathrm{L})\end{array}$ & $\begin{array}{c}\text { Zinc, } \\
\text { dissolved } \\
(\mu \mathrm{g} / \mathrm{L})\end{array}$ & $\begin{array}{l}\text { Sediment, } \\
\text { suspended } \\
\text { (mg/L) }\end{array}$ & $\begin{array}{c}\text { Sediment } \\
\text { discharge, } \\
\text { suspended } \\
\text { (ton/d) }\end{array}$ & $\begin{array}{l}\text { Sediment, } \\
\text { suspended } \\
\text { (percent } \\
\text { finer than } \\
0.062 \mathrm{~mm} \text { ) }\end{array}$ \\
\hline \multicolumn{9}{|l|}{ NOV 1998} \\
\hline $17 \ldots$ & $<0.5$ & 40 & 40 & $<10$ & $<20$ & 2 & 0.04 & 59 \\
\hline \multicolumn{9}{|l|}{ FEB 1999} \\
\hline $22 \ldots$ & $<.5$ & 40 & 38 & $<10$ & $<20$ & 5 & .08 & 94 \\
\hline \multicolumn{9}{|l|}{$A P R$} \\
\hline $27 \ldots$ & .5 & 60 & 35 & E21 & $<20$ & 39 & 1.5 & 92 \\
\hline \multicolumn{9}{|l|}{ MAY } \\
\hline $12 \ldots$ & $<.5$ & 41 & 29 & $<40$ & $<20$ & 4 & .16 & 97 \\
\hline $30 \ldots$ & $<.5$ & 110 & 62 & $<40$ & $<20$ & 29 & 2.4 & 81 \\
\hline \multicolumn{9}{|l|}{ JUN } \\
\hline $22 \ldots$ & $<5$ & 92 & 71 & $<40$ & $<20$ & 8 & .28 & 93 \\
\hline \multicolumn{9}{|l|}{ AUG } \\
\hline $12 \ldots$ & $<5$ & 51 & 39 & $<40$ & 3 & 8 & 18 & 84 \\
\hline
\end{tabular}

16 Water-quality, bed-sediment, and biological data (October 1998 through September 1999) and statistical summaries of data for streams in the Upper Clark Fork Basin, Montana 
Table 4. Water-quality data for the upper Clark Fork basin, Montana, October 1998 through September 1999 (Continued)

\begin{tabular}{|c|c|c|c|c|c|c|c|c|c|}
\hline Date & Time & $\begin{array}{c}\text { Streamflow, } \\
\text { instan- } \\
\text { taneous } \\
\left(\mathrm{ft}^{3} / \mathrm{s}\right)\end{array}$ & $\begin{array}{c}\text { Specific } \\
\text { conductance, } \\
\text { onsite } \\
(\mu S / \mathbf{c m})\end{array}$ & $\begin{array}{c}\text { pH, } \\
\text { onsite } \\
\text { (standard } \\
\text { units) }\end{array}$ & $\begin{array}{l}\text { Temper- } \\
\text { ature, } \\
\text { water } \\
\left({ }^{\circ} \mathrm{C}\right)\end{array}$ & $\begin{array}{c}\text { Hardness, } \\
\text { total } \\
\text { (mg/L as } \\
\left.\mathrm{CaCO}_{3}\right)\end{array}$ & $\begin{array}{l}\text { Calcium, } \\
\text { dissolved } \\
(\mathrm{mg} / \mathrm{L})\end{array}$ & $\begin{array}{l}\text { Magne- } \\
\text { sium, } \\
\text { dissolved } \\
\text { (mg/L) }\end{array}$ & $\begin{array}{c}\text { Arsenic, } \\
\text { total } \\
\text { recoverable } \\
(\mu \mathrm{g} / \mathrm{L})\end{array}$ \\
\hline \multicolumn{10}{|c|}{ NOV 1998} \\
\hline $17 \ldots$ & 1000 & 28 & 503 & 7.6 & 7.0 & 160 & 46 & 12 & 10 \\
\hline \multicolumn{10}{|l|}{ FEB 1999} \\
\hline $22 \ldots$ & 1115 & 21 & 514 & 7.6 & 6.0 & 170 & 47 & 12 & 10 \\
\hline \multicolumn{10}{|l|}{ APR } \\
\hline $27 \ldots$ & 1135 & 30 & 422 & 7.7 & 10.0 & 140 & 40 & 10 & 9 \\
\hline \multicolumn{10}{|l|}{ MAY } \\
\hline $12 \ldots$ & 1340 & 30 & 414 & 7.8 & 10.5 & 140 & 39 & 9.7 & 8 \\
\hline $30 \ldots$ & 1150 & 75 & 300 & 7.7 & 8.5 & 110 & 31 & 7.4 & 20 \\
\hline \multicolumn{10}{|l|}{ JUN } \\
\hline $22 \ldots$ & 1015 & 29 & 447 & 7.6 & 14.0 & 150 & 42 & 10 & 11 \\
\hline \multicolumn{10}{|l|}{ AUG } \\
\hline $12 \ldots$ & 1145 & 30 & 457 & 7.7 & 16.0 & 150 & 44 & 11 & 11 \\
\hline
\end{tabular}

\begin{tabular}{|c|c|c|c|c|c|c|c|c|}
\hline Date & $\begin{array}{c}\text { Arsenic, } \\
\text { dissolved } \\
(\mu \mathrm{g} / \mathrm{L})\end{array}$ & $\begin{array}{c}\text { Cadmium, } \\
\text { total } \\
\text { recoverable } \\
(\mu \mathrm{g} / \mathrm{L})\end{array}$ & $\begin{array}{c}\text { Cadmium. } \\
\text { dissolved } \\
(\mu \mathrm{g} / \mathrm{L})\end{array}$ & $\begin{array}{c}\text { Copper, } \\
\text { total } \\
\text { recoverable } \\
(\mu \mathrm{g} / \mathrm{L})\end{array}$ & $\begin{array}{l}\text { Copper, } \\
\text { dissolved } \\
(\mu \mathrm{g} / \mathrm{L})\end{array}$ & $\begin{array}{c}\text { Iron, } \\
\text { total } \\
\text { recoverable } \\
(\mu \mathrm{g} / \mathrm{L})\end{array}$ & $\begin{array}{c}\begin{array}{c}\text { Iron, } \\
\text { dissolved } \\
(\mu \mathrm{g} / \mathrm{L})\end{array}\end{array}$ & $\begin{array}{c}\text { Lead, } \\
\text { total } \\
\text { recoverable } \\
(\mu \mathrm{g} / \mathrm{L})\end{array}$ \\
\hline \multicolumn{9}{|l|}{ NOV 1998} \\
\hline $17 \ldots$ & 6 & 2 & 2 & 73 & 14 & 650 & 35 & 9 \\
\hline \multicolumn{9}{|l|}{ FEB 1999} \\
\hline $22 \ldots$ & 6 & 2 & 9 & 82 & 12 & 820 & 34 & 10 \\
\hline \multicolumn{9}{|l|}{ APR } \\
\hline $27 \ldots$ & 6 & $<1$ & 7 & 41 & 13 & 670 & 74 & 5 \\
\hline \multicolumn{9}{|l|}{ MAY } \\
\hline $12 \ldots$ & 7 & $<1$ & 7 & 40 & 14 & 583 & 72 & 4 \\
\hline 30. & 13 & 2 & 9 & 130 & 51 & 1,330 & 120 & 20 \\
\hline \multicolumn{9}{|l|}{$\pi N$} \\
\hline $22 \ldots$ & 9 & 1 & 1 & 41 & 19 & 549 & 81 & 3 \\
\hline \multicolumn{9}{|l|}{ AUG } \\
\hline $12 \ldots$ & 8 & 1 & 1 & 55 & 33 & 335 & 23 & 5 \\
\hline
\end{tabular}

\begin{tabular}{|c|c|c|c|c|c|c|c|c|}
\hline Date & $\begin{array}{c}\text { Lead, } \\
\text { dissolved } \\
(\mu \mathrm{g} / \mathrm{L})\end{array}$ & $\begin{array}{c}\text { Manga- } \\
\text { nese, } \\
\text { total } \\
\text { recoverable } \\
(\mu \mathrm{g} / \mathrm{L})\end{array}$ & $\begin{array}{l}\text { Manga- } \\
\text { nese, } \\
\text { dissolved } \\
(\mu \mathrm{g} / \mathrm{L})\end{array}$ & $\begin{array}{c}\text { Zinc, } \\
\text { total } \\
\text { recoverable } \\
(\mu \mathrm{g} / \mathrm{L})\end{array}$ & $\begin{array}{l}\text { Zinc, } \\
\text { dissolved } \\
(\mu \mathrm{g} / \mathrm{L})\end{array}$ & $\begin{array}{c}\text { Sediment, } \\
\text { suspended } \\
\text { (mg/L) }\end{array}$ & $\begin{array}{l}\text { Sediment } \\
\text { discharge, } \\
\text { suspended } \\
\text { (ton/d) }\end{array}$ & $\begin{array}{c}\text { Sediment, } \\
\text { suspended } \\
\text { (percent } \\
\text { finer than } \\
0.062 \mathrm{~mm} \text { ) }\end{array}$ \\
\hline \multicolumn{9}{|l|}{ NOV 1998} \\
\hline $17 \ldots$ & $<.5$ & 440 & 410 & 540 & 500 & 14 & 1.1 & 88 \\
\hline \multicolumn{9}{|l|}{ FEB 1999} \\
\hline $22 \ldots$ & $<.5$ & 410 & 390 & 500 & 430 & 20 & 1.1 & 82 \\
\hline \multicolumn{9}{|l|}{ APR } \\
\hline $27 \ldots$ & $<.5$ & 419 & 390 & 254 & 210 & 13 & 1.1 & 85 \\
\hline \multicolumn{9}{|l|}{ MAY } \\
\hline $12 \ldots$ & $<.5$ & 409 & 390 & 265 & 220 & 11 & .89 & 84 \\
\hline $30 \ldots$ & 9 & 358 & 300 & 352 & 240 & 35 & 7.1 & 83 \\
\hline \multicolumn{9}{|l|}{ JUN } \\
\hline $22 \ldots$ & $<.5$ & 520 & 510 & 398 & 370 & 6 & .47 & 94 \\
\hline \multicolumn{9}{|l|}{ AUG } \\
\hline $12 \ldots$ & $<.5$ & 245 & 234 & 396 & 337 & 6 & .49 & 98 \\
\hline
\end{tabular}


Table 4. Water-quality data for the upper Clark Fork basin, Montana, October 1998 through September 1999 (Continued)

\section{0--SILVER BOW CREEK AT OPPORTUNITY, MONT.}

\begin{tabular}{|c|c|c|c|c|c|c|c|c|c|}
\hline Date & Time & $\begin{array}{c}\text { Streamflow. } \\
\text { instan- } \\
\text { taneous } \\
\left(\mathrm{ft}^{3} / \mathrm{s}\right)\end{array}$ & $\begin{array}{c}\text { Specific } \\
\text { conductance, } \\
\text { onsite } \\
(\mu \mathrm{S} / \mathrm{cm})\end{array}$ & $\begin{array}{c}\text { pH, } \\
\text { onsite } \\
\text { (standard } \\
\text { units) }\end{array}$ & $\begin{array}{l}\text { Temper- } \\
\text { ature, } \\
\text { water } \\
\left({ }^{\circ} \mathrm{C}\right)\end{array}$ & $\begin{array}{c}\text { Hardness, } \\
\text { total } \\
(\mathrm{mg} / \mathrm{L} \text { as } \\
\left.\mathrm{CaCO}_{3}\right)\end{array}$ & $\begin{array}{l}\text { Calcium, } \\
\text { dissolved } \\
\text { (mg/L) }\end{array}$ & $\begin{array}{l}\text { Magne- } \\
\text { sium, } \\
\text { dissolved } \\
(\mathrm{mg} / \mathrm{L})\end{array}$ & $\begin{array}{c}\text { Arsenic, } \\
\text { total } \\
\text { recoverable } \\
(\mu \mathrm{g} / \mathrm{L})\end{array}$ \\
\hline \multicolumn{10}{|l|}{ NOV 1998} \\
\hline $17 \ldots$ & 1120 & 45 & 486 & 84 & 25 & 170 & 50 & 11 & 14 \\
\hline \multicolumn{10}{|l|}{ FEB 1999} \\
\hline $22 \ldots$ & 1245 & 36 & 517 & 8.2 & 1.5 & 180 & 54 & 12 & 19 \\
\hline \multicolumn{10}{|l|}{ APR } \\
\hline $27 \ldots$ & 1245 & 72 & 348 & 8.8 & 10.5 & 130 & 38 & 8.2 & 16 \\
\hline \multicolumn{10}{|l|}{ MAY } \\
\hline $12 \ldots$ & 1220 & 67 & 369 & 8.6 & 9.5 & 130 & 39 & 8.4 & 12 \\
\hline $30 \ldots$ & 1330 & 189 & 330 & 8.0 & 5.0 & 130 & 38 & 74 & 120 \\
\hline \multicolumn{10}{|l|}{ JUN } \\
\hline $04 \ldots$ & 0940 & 207 & 266 & 7.9 & 8.5 & 98 & 30 & 5.6 & 60 \\
\hline $22 \ldots$ & 1125 & 88 & 329 & 8.3 & 11.5 & 130 & 38 & 78 & 13 \\
\hline \multicolumn{10}{|l|}{ AUG } \\
\hline
\end{tabular}

\begin{tabular}{|c|c|c|c|c|c|c|c|c|}
\hline Date & $\begin{array}{c}\text { Arsenic, } \\
\text { dissolved } \\
(\mu \mathrm{g} / \mathrm{L})\end{array}$ & $\begin{array}{l}\text { Cadmium, } \\
\text { total } \\
\text { recoverable } \\
(\mu \mathrm{g} / \mathrm{L})\end{array}$ & $\begin{array}{c}\text { Cadmium, } \\
\text { dissolved } \\
(\mu \mathrm{g} / \mathrm{L})\end{array}$ & $\begin{array}{c}\text { Copper, } \\
\text { total } \\
\text { recoverable } \\
(\mu g / L)\end{array}$ & $\begin{array}{c}\text { Copper, } \\
\text { dissolved } \\
(\mu \mathrm{g} / \mathrm{L})\end{array}$ & $\begin{array}{c}\text { Iron, } \\
\text { total } \\
\text { recoverable } \\
(\mu \mathrm{g} / \mathrm{L})\end{array}$ & $\begin{array}{c}\text { Iron, } \\
\text { dissolved } \\
(\mu \mathrm{g} / \mathrm{L})\end{array}$ & $\begin{array}{c}\text { Lead, } \\
\text { total } \\
\text { recoverable } \\
(\mu \mathrm{g} / \mathrm{L})\end{array}$ \\
\hline \multicolumn{9}{|l|}{ NOV 1998} \\
\hline $17 \ldots$ & 8 & 2 & 1 & 100 & 37 & 490 & 10 & 12 \\
\hline \multicolumn{9}{|l|}{ FEB 1999} \\
\hline $22 \ldots$ & 10 & 2 & 2 & 160 & 32 & 910 & E9 & 22 \\
\hline \multicolumn{9}{|l|}{ APR } \\
\hline $27 \ldots$ & 9 & 1 & .5 & 96 & 44 & 782 & 26 & 15 \\
\hline \multicolumn{9}{|l|}{ MAY } \\
\hline $12 \ldots$ & 10 & 1 & 6 & 83 & 34 & 608 & 22 & 10 \\
\hline $30 \ldots$ & 11 & 7 & .9 & 1,200 & 61 & 9,320 & 100 & 310 \\
\hline \multicolumn{9}{|l|}{ JUN } \\
\hline $04 \ldots$ & 10 & 4 & 1 & 620 & 62 & 4,990 & 120 & 130 \\
\hline $22 \ldots$ & 9 & $<1$ & .7 & 80 & 34 & 478 & 19 & 9 \\
\hline \multicolumn{9}{|l|}{ AUG } \\
\hline
\end{tabular}

\begin{tabular}{|c|c|c|c|c|c|c|c|c|}
\hline Date & $\begin{array}{l}\text { Lead, } \\
\text { dissolved } \\
(\mu \mathrm{g} / \mathrm{L})\end{array}$ & $\begin{array}{l}\text { Manga- } \\
\text { nese, } \\
\text { total } \\
\text { recoverable } \\
(\mu \mathrm{g} / \mathrm{L})\end{array}$ & $\begin{array}{l}\text { Manga- } \\
\text { nese, } \\
\text { dissolved } \\
(\mu \mathrm{g} / \mathrm{L})\end{array}$ & $\begin{array}{c}\text { Zinc, } \\
\text { total } \\
\text { recoverable } \\
(\mu \mathrm{g} / \mathrm{L})\end{array}$ & $\begin{array}{c}\text { Zinc, } \\
\text { dissolved } \\
(\mu \mathrm{g} / \mathrm{L})\end{array}$ & $\begin{array}{l}\text { Sediment, } \\
\text { suspended } \\
\text { (mg/L) }\end{array}$ & $\begin{array}{c}\text { Sediment } \\
\text { discharge, } \\
\text { suspended } \\
\text { (ton/d) }\end{array}$ & $\begin{array}{c}\text { Sediment, } \\
\text { suspended } \\
\text { (percent } \\
\text { finer than } \\
0.062 \mathrm{~mm} \text { ) }\end{array}$ \\
\hline \multicolumn{9}{|l|}{ NOV 1998} \\
\hline $17 \ldots$ & $<5$ & 490 & 470 & 530 & 430 & 9 & 11 & 86 \\
\hline \multicolumn{9}{|l|}{ FEB 1999} \\
\hline 22. & $<.5$ & 570 & 520 & 560 & 430 & 21 & 2.0 & 87 \\
\hline \multicolumn{9}{|l|}{ APR } \\
\hline $27 \ldots$ & $<.5$ & 359 & 280 & 250 & 100 & 19 & 3.7 & 91 \\
\hline \multicolumn{9}{|l|}{ MAY } \\
\hline 12. & $<.5$ & 386 & 330 & 260 & 130 & 13 & 2.4 & 93 \\
\hline $30 \ldots$ & 2 & 1,690 & 650 & 1,720 & 290 & 491 & 251 & 53 \\
\hline \multicolumn{9}{|l|}{ JUN } \\
\hline $04 .$. & 3 & 884 & 420 & 1,000 & 290 & 196 & 110 & 47 \\
\hline $22 \ldots$ & $<.5$ & 314 & 280 & 237 & 160 & 13 & 31 & 74 \\
\hline \multicolumn{9}{|l|}{ AUG } \\
\hline $12 \ldots$ & .5 & 598 & 430 & 533 & 94 & 32 & 3.2 & 94 \\
\hline
\end{tabular}

18 Water-quality, bed-sediment, and biological data (October 1998 through September 1999) and statistical summaries of data for streams in the Upper Clark Fork Basin, Montana 
Table 4. Water-quality data for the upper Clark Fork basin, Montana, October 1998 through September 1999 (Continued)

12323750--SILVER BOW CREEK AT WARM SPRINGS, MONT.

\begin{tabular}{|c|c|c|c|c|c|c|c|c|c|}
\hline Date & Time & $\begin{array}{c}\text { Streamflow, } \\
\text { instan- } \\
\text { taneous } \\
\left(\mathrm{ft}^{3} / \mathrm{s}\right)\end{array}$ & $\begin{array}{c}\text { Specific } \\
\text { conductance, } \\
\text { onsite } \\
(\mu \mathrm{S} / \mathrm{cm})\end{array}$ & $\begin{array}{c}\text { pH, } \\
\text { onsite } \\
\text { (standard } \\
\text { units) }\end{array}$ & $\begin{array}{c}\text { Temper- } \\
\text { ature, } \\
\text { water } \\
\left({ }^{\circ} \mathrm{C}\right)\end{array}$ & $\begin{array}{c}\text { Hardness, } \\
\text { total } \\
(\mathrm{mg} / \mathrm{L} \text { as } \\
\left.\mathrm{CaCO}_{3}\right)\end{array}$ & $\begin{array}{c}\text { Calcium, } \\
\text { dissolved } \\
(\mathrm{mg} / \mathrm{L})\end{array}$ & $\begin{array}{l}\text { Magne- } \\
\text { sium, } \\
\text { dissolved } \\
(\mathrm{mg} / \mathrm{L})\end{array}$ & $\begin{array}{c}\text { Arsenic, } \\
\text { total } \\
\text { recoverable } \\
(\mu \mathrm{g} / \mathrm{L})\end{array}$ \\
\hline \multicolumn{10}{|l|}{ NOV $19 \overline{98}$} \\
\hline $17 \ldots$ & 1250 & 74 & 535 & 8.9 & 35 & 220 & 62 & 16 & 19 \\
\hline \multicolumn{10}{|l|}{ FEB 1999} \\
\hline 22 & 1325 & 59 & 602 & 8.8 & 3.5 & 250 & 73 & 17 & 13 \\
\hline \multicolumn{10}{|l|}{ APR } \\
\hline 27. & 1345 & 112 & 427 & 8.5 & 12.0 & 160 & 47 & 11 & 25 \\
\hline \multicolumn{10}{|l|}{ MAY } \\
\hline $11 \ldots$ & 0950 & 128 & 425 & 8.4 & 7.0 & 160 & 45 & 11 & 22 \\
\hline 27. & 1530 & 263 & 287 & 8.8 & 15.0 & 110 & 31 & 7.5 & 38 \\
\hline \multicolumn{10}{|l|}{ JUN } \\
\hline 10. & 1040 & 288 & 306 & 9.0 & 8.0 & 120 & 35 & 7.9 & 27 \\
\hline 22 & 1320 & 252 & 249 & 9.1 & 13.0 & 97 & 28 & 6.6 & 26 \\
\hline \multicolumn{10}{|l|}{ AUG } \\
\hline $13 \ldots$ & 1115 & 53 & 425 & 9.3 & 17.0 & 180 & 51 & 12 & 32 \\
\hline
\end{tabular}

\begin{tabular}{|c|c|c|c|c|c|c|c|c|}
\hline Date & $\begin{array}{c}\text { Arsenic, } \\
\text { dissolved } \\
(\mu \mathrm{g} / \mathrm{L})\end{array}$ & $\begin{array}{c}\text { Cadmium, } \\
\text { total } \\
\text { recoverable } \\
(\mu \mathrm{g} / \mathrm{L})\end{array}$ & $\begin{array}{c}\text { Cadmium, } \\
\text { dissolved } \\
(\mu \mathrm{g} / \mathrm{L})\end{array}$ & $\begin{array}{c}\text { Copper, } \\
\text { total } \\
\text { recoverable } \\
(\mu \mathrm{g} / \mathrm{L})\end{array}$ & $\begin{array}{l}\text { Copper, } \\
\text { dissolved } \\
(\mu \mathrm{g} / \mathrm{L})\end{array}$ & $\begin{array}{c}\text { Iron, } \\
\text { total } \\
\text { recoverable } \\
(\mu \mathrm{g} / \mathrm{L})\end{array}$ & $\begin{array}{c}\text { Iron, } \\
\text { dissolved } \\
(\mu \mathrm{g} / \mathrm{L})\end{array}$ & $\begin{array}{c}\text { Lead, } \\
\text { total } \\
\text { recoverable } \\
(\mu \mathrm{g} / \mathrm{L})\end{array}$ \\
\hline \multicolumn{9}{|c|}{ NOV 1998} \\
\hline $17 \ldots$ & 15 & $<1$ & $<1$ & 6 & 4 & 170 & $<10$ & $<1$ \\
\hline \multicolumn{9}{|l|}{ FEB 1999} \\
\hline $22 \ldots$ & 11 & $<1$ & $<.1$ & 13 & 7 & 170 & $<10$ & $<1$ \\
\hline \multicolumn{9}{|l|}{ APR } \\
\hline $27 \ldots$ & 21 & $<1$ & $<.1$ & 13 & 6 & 368 & 19 & 2 \\
\hline \multicolumn{9}{|l|}{ MAY } \\
\hline $11 \ldots$ & 22 & $<1$ & $<.1$ & 11 & 5 & 312 & 19 & 2 \\
\hline $27 \ldots$ & 28 & $<1$ & $<.1$ & 22 & 7 & 909 & 35 & 5 \\
\hline \multicolumn{9}{|l|}{ JUN } \\
\hline $10 \ldots$ & 23 & $<1$ & $<1$ & 14 & 8 & 269 & 22 & 2 \\
\hline $22 \ldots$ & 25 & $<1$ & $<.1$ & 11 & 7 & 206 & 24 & 1 \\
\hline \multicolumn{9}{|l|}{ AUG } \\
\hline $13 \ldots$ & 32 & $<1$ & $<1$ & 5 & 3 & 137 & 19 & $<1$ \\
\hline
\end{tabular}

\begin{tabular}{|c|c|c|c|c|c|c|c|c|}
\hline Date & $\begin{array}{c}\text { Lead, } \\
\text { dissolved } \\
(\mu \mathrm{g} / \mathrm{L})\end{array}$ & $\begin{array}{l}\text { Manga- } \\
\text { nese, } \\
\text { total } \\
\text { recoverable } \\
(\mu \mathrm{g} / \mathrm{L})\end{array}$ & $\begin{array}{c}\text { Manga- } \\
\text { nese, } \\
\text { dissolved } \\
(\mu \mathrm{g} / \mathrm{L})\end{array}$ & $\begin{array}{c}\text { Zinc, } \\
\text { total } \\
\text { recoverable } \\
(\mu \mathrm{g} / \mathrm{L})\end{array}$ & $\begin{array}{c}\begin{array}{c}\text { Zinc, } \\
\text { dissolved } \\
(\mu \mathrm{g} / \mathrm{L})\end{array} \\
\end{array}$ & $\begin{array}{l}\text { Sediment, } \\
\text { suspended } \\
\text { (mg/L) }\end{array}$ & $\begin{array}{c}\text { Sediment } \\
\text { discharge, } \\
\text { suspended } \\
\text { (ton/d) }\end{array}$ & $\begin{array}{c}\text { Sediment, } \\
\text { suspended } \\
\text { (percent } \\
\text { finer than } \\
0.062 \mathrm{~mm} \text { ) }\end{array}$ \\
\hline \multicolumn{9}{|l|}{ NOV 1998} \\
\hline $17 \ldots$ & $<.5$ & 90 & 40 & 10 & $<20$ & 3 & 0.60 & 91 \\
\hline \multicolumn{9}{|l|}{ FEB 1999} \\
\hline 22. & $<.5$ & 200 & 120 & 40 & E17 & 5 & .80 & 82 \\
\hline \multicolumn{9}{|l|}{ APR } \\
\hline $27 \ldots$ & $<.5$ & 183 & 120 & E30 & $<20$ & 12 & 3.6 & 95 \\
\hline \multicolumn{9}{|l|}{ MAY } \\
\hline $11 \ldots$ & $<, 5$ & 111 & 76 & $<40$ & E8 & 10 & 3.5 & 92 \\
\hline $27 \ldots$ & $<.5$ & 120 & 34 & E23 & $<20$ & 44 & 31 & 86 \\
\hline \multicolumn{9}{|l|}{ JUN } \\
\hline 10. & $<.5$ & 135 & 97 & $<40$ & $<20$ & 7 & 5.4 & 86 \\
\hline 22. & $<.5$ & 145 & 73 & $<40$ & $<20$ & 7 & 4.8 & 87 \\
\hline \multicolumn{9}{|l|}{ AUG } \\
\hline $13 \ldots$ & $<.5$ & 121 & 58 & $<40$ & 2 & 3 & .43 & 87 \\
\hline
\end{tabular}


Table 4. Water-quality data for the upper Clark Fork basin. Montana, October 1998 through September 1999 (Continued)

12323770--WARM SPRINGS CREEK AT WARM SPRINGS, MONT.

\begin{tabular}{|c|c|c|c|c|c|c|c|c|c|}
\hline Date & Time & $\begin{array}{c}\text { Streamflow, } \\
\text { instan- } \\
\text { tanęous } \\
\left(\mathrm{ft}^{3} / \mathrm{s}\right)\end{array}$ & $\begin{array}{c}\text { Specific } \\
\text { conductance, } \\
\text { onsite } \\
(\mu \mathrm{S} / \mathrm{cm})\end{array}$ & $\begin{array}{c}\text { pH, } \\
\text { onsite } \\
\text { (standard } \\
\text { units) }\end{array}$ & $\begin{array}{c}\text { Temper- } \\
\text { ature, } \\
\text { water } \\
\left({ }^{\circ} \mathrm{C}\right)\end{array}$ & $\begin{array}{c}\text { Hardness, } \\
\text { total } \\
(\mathrm{mg} / \mathrm{L} \text { as } \\
\left.\mathrm{CaCO}_{3}\right)\end{array}$ & $\begin{array}{c}\text { Calcium, } \\
\text { dissolved } \\
(m g / L)\end{array}$ & $\begin{array}{l}\text { Magne- } \\
\text { sium, } \\
\text { dissolved } \\
(\mathrm{mg} / \mathrm{L})\end{array}$ & $\begin{array}{c}\text { Arsenic, } \\
\text { total } \\
\text { recoverable } \\
(\mu \mathrm{g} / \mathrm{L})\end{array}$ \\
\hline \multicolumn{10}{|l|}{ NOV 1998} \\
\hline 17. & 1220 & 53 & 342 & 8.6 & 3.0 & 160 & 50 & 9.9 & 4 \\
\hline \multicolumn{10}{|l|}{ APR 1999} \\
\hline $27 \ldots$ & 1430 & 48 & 338 & 8.5 & 11.0 & 160 & 49 & 10 & 5 \\
\hline \multicolumn{10}{|l|}{ MAY } \\
\hline 27. & 1430 & 230 & 156 & 8.2 & 10.5 & 72 & 22 & 39 & 12 \\
\hline \multicolumn{10}{|l|}{ JUN } \\
\hline $10 \ldots$ & 1125 & 116 & 228 & 8.2 & 7.0 & 110 & 33 & 6.2 & 5 \\
\hline $22 \ldots$ & 1210 & 246 & 144 & 8.0 & 9.5 & 66 & 20 & 3.6 & 5 \\
\hline \multicolumn{10}{|l|}{ AUG } \\
\hline $12 \ldots$ & 1415 & 47 & 266 & 8.7 & 15.0 & 120 & 37 & 7.1 & 6 \\
\hline
\end{tabular}

\begin{tabular}{|c|c|c|c|c|c|c|c|c|}
\hline Date & $\begin{array}{c}\text { Arsenic, } \\
\text { dissolved } \\
(\mu \mathrm{g} / \mathrm{L})\end{array}$ & $\begin{array}{c}\text { Cadmium, } \\
\text { total } \\
\text { recoverable } \\
(\mu \mathrm{g} / \mathrm{L})\end{array}$ & $\begin{array}{l}\text { Cadmium, } \\
\text { dissolved } \\
(\mu \mathrm{g} / \mathrm{L})\end{array}$ & $\begin{array}{c}\text { Copper, } \\
\text { total } \\
\text { recoverable } \\
(\mu \mathrm{g} / \mathrm{L})\end{array}$ & $\begin{array}{r}\text { Copper, } \\
\text { dissolved } \\
(\mu \mathrm{g} / \mathrm{L})\end{array}$ & $\begin{array}{l}\text { Iron, } \\
\text { total } \\
\text { recoverable } \\
(\mu \mathrm{g} / \mathrm{L})\end{array}$ & $\begin{array}{c}\text { Iron, } \\
\text { dissolved } \\
(\mu \mathrm{g} / \mathrm{L})\end{array}$ & $\begin{array}{c}\text { Lead, } \\
\text { total } \\
\text { recoverable } \\
(\mu \mathrm{g} / \mathrm{L})\end{array}$ \\
\hline \multicolumn{9}{|c|}{ NOV 1998} \\
\hline $17 \ldots$ & 2 & $<1$ & $<.1$ & 2 & 2 & 40 & E7 & $<1$ \\
\hline \multicolumn{9}{|c|}{ APR 1999} \\
\hline $27 \ldots$ & 4 & $<1$ & $<.1$ & 9 & 2 & 122 & E7 & $<1$ \\
\hline \multicolumn{9}{|l|}{ MAY } \\
\hline $27 \ldots$ & 6 & $<1$ & $<1$ & 61 & 5 & 1,070 & 15 & 6 \\
\hline \multicolumn{9}{|l|}{ JUN } \\
\hline $10 \ldots$ & 4 & $<1$ & $<.1$ & 12 & 2 & 218 & 12 & 1 \\
\hline $22 \ldots$ & 4 & $<1$ & $<.1$ & 19 & 3 & 461 & 11 & 2 \\
\hline \multicolumn{9}{|l|}{ AUG } \\
\hline $12 \ldots$ & 5 & $<1$ & $<.1$ & 8 & 2 & 84 & 13 & $<1$ \\
\hline
\end{tabular}

\begin{tabular}{|c|c|c|c|c|c|c|c|c|}
\hline Date & $\begin{array}{c}\text { Lead, } \\
\text { dissolved } \\
(\mu \mathrm{g} / \mathrm{L})\end{array}$ & $\begin{array}{c}\text { Manga- } \\
\text { nese, } \\
\text { total } \\
\text { recoverable } \\
(\mu \mathrm{g} / \mathrm{L})\end{array}$ & $\begin{array}{l}\text { Manga- } \\
\text { nese, } \\
\text { dissolved } \\
(\mu \mathrm{g} / \mathrm{L})\end{array}$ & $\begin{array}{c}\text { Zinc, } \\
\text { total } \\
\text { recoverable } \\
(\mu \mathrm{g} / \mathrm{L})\end{array}$ & $\begin{array}{c}\text { Zinc, } \\
\text { dissolved } \\
(\mu \mathrm{g} / \mathrm{L})\end{array}$ & $\begin{array}{c}\text { Sediment, } \\
\text { suspended } \\
\text { (mg/L) }\end{array}$ & $\begin{array}{c}\text { Sediment } \\
\text { discharge, } \\
\text { suspended } \\
\text { (ton/d) }\end{array}$ & $\begin{array}{c}\text { Sediment, } \\
\text { suspended } \\
\text { (percent } \\
\text { finer than } \\
0.062 \mathrm{~mm} \text { ) }\end{array}$ \\
\hline \multicolumn{9}{|c|}{ NOV 1998} \\
\hline 17. & $<5$ & 210 & 210 & $<10$ & $<20$ & 2 & 29 & 79 \\
\hline \multicolumn{9}{|c|}{ APR 1999} \\
\hline 27. & $<5$ & 231 & 170 & $<40$ & $<20$ & 8 & 10 & 78 \\
\hline \multicolumn{9}{|l|}{ MAY } \\
\hline $27 \ldots$ & $<.5$ & 273 & 61 & E29 & $<20$ & 62 & 39 & 71 \\
\hline \multicolumn{9}{|l|}{ JUN } \\
\hline $10 \ldots$ & $<.5$ & 131 & 97 & $<40$ & $<20$ & 11 & 3.4 & 68 \\
\hline $22 \ldots$ & $<.5$ & 93 & 41 & $<40$ & $<20$ & 24 & 16 & 57 \\
\hline \multicolumn{9}{|l|}{ AUG } \\
\hline $12 \ldots$ & $<.5$ & 110 & 80 & $<40$ & $<1$ & 4 & .51 & 74 \\
\hline
\end{tabular}


Table 4. Water-quality data for the upper Clark Fork basin, Montana, October 1998 through September 1999 (Continued)

12323800-CLARK FORK NEAR GALEN, MONT.

\begin{tabular}{|c|c|c|c|c|c|c|c|c|c|}
\hline Date & Time & $\begin{array}{l}\text { Streamflow, } \\
\text { instan- } \\
\text { taneous } \\
\left(\mathrm{ft}^{3} / \mathrm{s}\right)\end{array}$ & $\begin{array}{c}\text { Specific } \\
\text { conductance, } \\
\text { onsite } \\
(\mu \mathrm{S} / \mathrm{cm})\end{array}$ & $\begin{array}{c}\text { pH, } \\
\text { onsite } \\
\text { (standard } \\
\text { units) }\end{array}$ & $\begin{array}{l}\text { Temper- } \\
\text { ature, } \\
\text { water } \\
\left({ }^{\circ} \mathrm{C}\right)\end{array}$ & $\begin{array}{c}\text { Hardness, } \\
\text { total } \\
(\mathrm{mg} / \mathrm{L} \text { as } \\
\left.\mathrm{CaCO}_{3}\right)\end{array}$ & $\begin{array}{c}\text { Calcium, } \\
\text { dissolved } \\
(\mathrm{mg} / \mathrm{L})\end{array}$ & $\begin{array}{l}\text { Magne- } \\
\text { sium, } \\
\text { dissolved } \\
(\mathrm{mg} / \mathrm{L})\end{array}$ & $\begin{array}{c}\text { Arsenic, } \\
\text { total } \\
\text { recoverabl } \\
(\mu \mathrm{g} / \mathrm{L})\end{array}$ \\
\hline \multicolumn{10}{|c|}{ NOV 1998} \\
\hline $17 \ldots$ & 1355 & 124 & 460 & 8.8 & 4.0 & 190 & 55 & 13 & 12 \\
\hline \multicolumn{10}{|l|}{ FEB 1999} \\
\hline $22 \ldots$ & 1420 & 113 & 517 & 8.7 & 4.5 & 230 & 66 & 15 & 9 \\
\hline \multicolumn{10}{|l|}{ APR } \\
\hline $27 \ldots$ & 1520 & 158 & 398 & 8.7 & 12.0 & 170 & 48 & 11 & 18 \\
\hline \multicolumn{10}{|l|}{ MAY } \\
\hline $11 \ldots$ & 1040 & 183 & 401 & 8.5 & 8.0 & 160 & 48 & 11 & 17 \\
\hline $27 \ldots$ & 1345 & 499 & 229 & 8.4 & 12.0 & 94 & 28 & 5.9 & 31 \\
\hline \multicolumn{10}{|l|}{ JUN } \\
\hline $10 \ldots$ & 1155 & 435 & 286 & 8.8 & 9.0 & 120 & 34 & 7.3 & 22 \\
\hline $22 \ldots$ & 1415 & 522 & 197 & 8.8 & 12.5 & 81 & 24 & 5.1 & 17 \\
\hline \multicolumn{10}{|l|}{ AUG } \\
\hline $13 \ldots$ & 1145 & 91 & 373 & 8.8 & 15.0 & 160 & 48 & 10 & 20 \\
\hline
\end{tabular}

\begin{tabular}{|c|c|c|c|c|c|c|c|c|}
\hline Date & $\begin{array}{c}\text { Arsenic, } \\
\text { dissolved } \\
(\mu \mathrm{g} / \mathrm{L})\end{array}$ & $\begin{array}{l}\text { Cadminm, } \\
\text { total } \\
\text { recoverable } \\
(\mu \mathrm{g} / \mathrm{L})\end{array}$ & $\begin{array}{c}\text { Cadmium, } \\
\text { dissolved } \\
(\mu \mathrm{g} / \mathrm{L})\end{array}$ & $\begin{array}{c}\text { Copper, } \\
\text { total } \\
\text { recoverable } \\
(\mu \mathrm{g} / \mathrm{L})\end{array}$ & $\begin{array}{c}\text { Copper, } \\
\text { dissolved } \\
(\mu \mathrm{g} / \mathrm{L})\end{array}$ & $\begin{array}{l}\text { Iron, } \\
\text { total } \\
\text { recoverable } \\
(\mu \mathrm{g} / \mathrm{L})\end{array}$ & $\begin{array}{c}\text { Iron, } \\
\text { dissolved } \\
(\mu \mathrm{g} / \mathrm{L})\end{array}$ & $\begin{array}{c}\text { Lead, } \\
\text { total } \\
\text { recoverable } \\
(\mu \mathrm{g} / \mathrm{L})\end{array}$ \\
\hline \multicolumn{9}{|l|}{ NOV 1998} \\
\hline 17. & 9 & $<1$ & $<1$ & 5 & 3 & 110 & $<10$ & $<1$ \\
\hline \multicolumn{9}{|l|}{ FEB 1999} \\
\hline 22 & 7 & $<1$ & $<.1$ & 12 & 5 & 140 & $<10$ & $<1$ \\
\hline \multicolumn{9}{|l|}{ APR } \\
\hline $27 .$. & 15 & $<1$ & 1 & 15 & 5 & 296 & E8 & 2 \\
\hline \multicolumn{9}{|l|}{ MAY } \\
\hline $11 \ldots$ & 17 & $<1$ & $<.1$ & 15 & 5 & 294 & 12 & 2 \\
\hline $27 .$. & 17 & $<1$ & $<.1$ & 71 & 7 & 1,480 & 25 & 9 \\
\hline \multicolumn{9}{|l|}{ JUN } \\
\hline 10. & 17 & $<1$ & $<.1$ & 17 & 6 & 293 & 13 & 2 \\
\hline $22 .$. & 14 & $<1$ & $<.1$ & 19 & 5 & 355 & 16 & 2 \\
\hline \multicolumn{9}{|l|}{ AUG } \\
\hline $13 \ldots$ & 19 & $<1$ & $<.1$ & 12 & 3 & 190 & 14 & 1 \\
\hline
\end{tabular}

\begin{tabular}{|c|c|c|c|c|c|c|c|c|}
\hline Date & $\begin{array}{c}\text { Lead, } \\
\text { dissolved } \\
(\mu \mathrm{g} / \mathrm{L})\end{array}$ & $\begin{array}{l}\text { Manga- } \\
\text { nese, } \\
\text { total } \\
\text { recoverable } \\
(\mu \mathrm{g} / \mathrm{L})\end{array}$ & $\begin{array}{l}\text { Manga- } \\
\text { nese, } \\
\text { dissolved } \\
(\mu \mathrm{g} / \mathrm{L})\end{array}$ & $\begin{array}{c}\text { Zinc, } \\
\text { total } \\
\text { recoverable } \\
(\mu \mathrm{g} / \mathrm{L})\end{array}$ & $\begin{array}{c}\text { Zinc, } \\
\text { dissolved } \\
(\mu \mathrm{g} / \mathrm{L})\end{array}$ & $\begin{array}{l}\text { Sediment, } \\
\text { suspended } \\
(\mathrm{mg} / \mathrm{L})\end{array}$ & $\begin{array}{c}\text { Sediment } \\
\text { discharge, } \\
\text { suspended } \\
\text { (ton/d) }\end{array}$ & $\begin{array}{c}\text { Sediment, } \\
\text { suspended } \\
\text { (percent } \\
\text { finer than } \\
0.062 \mathrm{~mm} \text { ) }\end{array}$ \\
\hline \multicolumn{9}{|c|}{ NOV 1998} \\
\hline $17 \ldots$ & $<.5$ & 120 & 89 & $<10$ & $<20$ & 3 & 1.0 & 86 \\
\hline \multicolumn{9}{|l|}{ FEB 1999} \\
\hline $22 \ldots$ & $<.5$ & 200 & 150 & 20 & $<20$ & 5 & 1.5 & 84 \\
\hline \multicolumn{9}{|l|}{ APR } \\
\hline $27 \ldots$ & $<.5$ & 179 & 89 & $<40$ & $<20$ & 10 & 4.3 & 83 \\
\hline \multicolumn{9}{|l|}{ MAY } \\
\hline $11 \ldots$ & $<.5$ & 139 & 82 & E25 & $<20$ & 10 & 49 & 84 \\
\hline $27 \ldots$ & $<5$ & 303 & 51 & 56 & $<20$ & 77 & 104 & 73 \\
\hline \multicolumn{9}{|l|}{ JUN } \\
\hline $10 \ldots$ & $<.5$ & 130 & 75 & $<40$ & $<20$ & 18 & 21 & 50 \\
\hline $22 \ldots$ & $<.5$ & 116 & 40 & $<40$ & $<20$ & 16 & 23 & 66 \\
\hline \multicolumn{9}{|l|}{ AUG } \\
\hline $13 \ldots$ & $<5$ & 143 & 54 & $<40$ & 2 & 8 & 2.0 & 83 \\
\hline
\end{tabular}


Table 4. Water-quality data for the upper Clark Fork basin, Montana, October 1998 through September 1999 (Continued) 12324200--CLARK FORK AT DEER LODGE, MONT.

\begin{tabular}{|c|c|c|c|c|c|c|c|c|c|}
\hline Date & Time & $\begin{array}{c}\text { Streamflow, } \\
\text { instan- } \\
\text { taneous } \\
\left(\mathrm{ft}^{3} / \mathrm{s}\right)\end{array}$ & $\begin{array}{c}\text { Specific } \\
\text { conductance, } \\
\text { onsite } \\
(\mu \mathrm{S} / \mathrm{cm})\end{array}$ & $\begin{array}{c}\text { pH, } \\
\text { onsite } \\
\text { (standard } \\
\text { units) }\end{array}$ & $\begin{array}{l}\text { Temper- } \\
\text { ature, } \\
\text { water } \\
\left({ }^{\circ} \mathrm{C}\right)\end{array}$ & $\begin{array}{c}\text { Hardness, } \\
\text { total } \\
(\mathrm{mg} / \mathrm{L} \text { as } \\
\left.\mathrm{CaCO}_{3}\right)\end{array}$ & $\begin{array}{c}\text { Calcium, } \\
\text { dissolved } \\
\text { (mg/L) }\end{array}$ & $\begin{array}{l}\text { Magne- } \\
\text { sium, } \\
\text { dissolved } \\
(\mathrm{mg} / \mathrm{L})\end{array}$ & $\begin{array}{c}\text { Arsenic, } \\
\text { total } \\
\text { recoverable } \\
(\mu \mathrm{g} / \mathrm{L})\end{array}$ \\
\hline \multicolumn{10}{|l|}{ NOV 1998} \\
\hline $17 \ldots$ & 1505 & 286 & 473 & 8.4 & 4.0 & 210 & 60 & 14 & 12 \\
\hline \multicolumn{10}{|l|}{ FEB 1999} \\
\hline $22 \ldots$ & 1540 & 225 & 502 & 8.6 & 4.0 & 230 & 67 & 15 & 9 \\
\hline \multicolumn{10}{|l|}{ APR } \\
\hline $27 \ldots$ & 1625 & 250 & 454 & 8.9 & 12.5 & 200 & 57 & 13 & 14 \\
\hline \multicolumn{10}{|l|}{ MAY } \\
\hline $11 \ldots$ & 1200 & 281 & 460 & 8.6 & 8.5 & 200 & 57 & 13 & 14 \\
\hline $27 \ldots$ & 1230 & 506 & 286 & 8.0 & 14.0 & 120 & 34 & 7.4 & 56 \\
\hline \multicolumn{10}{|l|}{ JUN } \\
\hline $10 \ldots$ & 0910 & 500 & 341 & 8.2 & 9,0 & 140 & 42 & 9.2 & 27 \\
\hline $22 \ldots$ & 1600 & 684 & 234 & 8.3 & 14.5 & 95 & 28 & 6.0 & 20 \\
\hline \multicolumn{10}{|l|}{ AUG } \\
\hline $13 \ldots$ & 1340 & 132 & 467 & 8.6 & 170 & 200 & 59 & 13 & 20 \\
\hline
\end{tabular}

\begin{tabular}{|c|c|c|c|c|c|c|c|c|}
\hline Date & $\begin{array}{l}\text { Arsenic, } \\
\text { dissolved } \\
(\mu \mathrm{g} / \mathrm{L})\end{array}$ & $\begin{array}{c}\text { Cadmium, } \\
\text { total } \\
\text { recoverable } \\
(\mu g / L)\end{array}$ & $\begin{array}{c}\text { Cadmium, } \\
\text { dissolved } \\
(\mu g / L)\end{array}$ & $\begin{array}{c}\text { Copper, } \\
\text { total } \\
\text { recoverable } \\
(\mu \mathrm{g} / \mathrm{L})\end{array}$ & $\begin{array}{l}\text { Copper, } \\
\text { dissolved } \\
(\mu \mathrm{g} / \mathrm{L})\end{array}$ & $\begin{array}{l}\text { Iron, } \\
\text { total } \\
\text { recoverable } \\
(\mu \mathrm{g} / \mathrm{L})\end{array}$ & $\begin{array}{c}\text { Iron, } \\
\text { dissolved } \\
(\mu \mathrm{g} / \mathrm{L})\end{array}$ & $\begin{array}{c}\text { Lead, } \\
\text { total } \\
\text { recoverable } \\
(\mu \mathrm{g} / \mathrm{L})\end{array}$ \\
\hline \multicolumn{9}{|l|}{ NOV 1998} \\
\hline $17 \ldots$ & 9 & $<1$ & $<.1$ & 16 & 4 & 280 & $<10$ & 2 \\
\hline \multicolumn{9}{|l|}{ FEB 1999} \\
\hline $22 \ldots$ & 7 & $<1$ & .1 & 16 & 5 & 220 & $<10$ & 2 \\
\hline \multicolumn{9}{|l|}{ APR } \\
\hline $27 \ldots$ & 12 & $<1$ & $<1$ & 20 & 7 & 339 & $<10$ & 2 \\
\hline \multicolumn{9}{|l|}{ MAY } \\
\hline $11 \ldots$ & 13 & $<1$ & $<.1$ & 24 & 6 & 408 & $<10$ & 3 \\
\hline $27 \ldots$ & 19 & I & $<.1$ & 260 & 11 & 3,750 & 23 & 31 \\
\hline \multicolumn{9}{|l|}{ JUN } \\
\hline $10 \ldots$ & 17 & $<1$ & $<1$ & 59 & 10 & 904 & 16 & 8 \\
\hline 22. & 15 & $<1$ & $<.1$ & 70 & 10 & 927 & 18 & 7 \\
\hline \multicolumn{9}{|l|}{ AUG } \\
\hline $13 \ldots$ & 18 & $<1$ & $<.1$ & 32 & 7 & 377 & E7 & 4 \\
\hline
\end{tabular}

\begin{tabular}{|c|c|c|c|c|c|c|c|c|}
\hline Date & $\begin{array}{l}\text { Lead, } \\
\text { dissolved } \\
(\mu \mathrm{g} / \mathrm{L})\end{array}$ & $\begin{array}{l}\text { Manga- } \\
\text { nese, } \\
\text { total } \\
\text { recoverable } \\
(\mu g / L)\end{array}$ & $\begin{array}{c}\text { Manga- } \\
\text { nese, } \\
\text { dissolved } \\
(\mu \mathrm{g} / \mathrm{L})\end{array}$ & $\begin{array}{c}\text { Zinc, } \\
\text { total } \\
\text { recoverable } \\
(\mu \mathrm{g} / \mathrm{L})\end{array}$ & $\begin{array}{c}\text { Zinc, } \\
\text { dissolved } \\
(\mu \mathrm{g} / \mathrm{L})\end{array}$ & $\begin{array}{l}\text { Sediment, } \\
\text { suspended } \\
\text { (mg/L) }\end{array}$ & $\begin{array}{c}\text { Sediment } \\
\text { discharge, } \\
\text { suspended } \\
\text { (ton/d) }\end{array}$ & $\begin{array}{l}\text { Sediment, } \\
\text { suspended } \\
\text { (percent } \\
\text { finer than } \\
0.062 \mathrm{~mm} \text { ) }\end{array}$ \\
\hline \multicolumn{9}{|c|}{ NOV 1998} \\
\hline $17 \ldots$ & $<.5$ & 70 & 24 & 20 & $<20$ & 15 & 12 & 60 \\
\hline \multicolumn{9}{|c|}{ FEB 1999} \\
\hline $22 \ldots$ & $<.5$ & 91 & 51 & 30 & $<10$ & 15 & 9.1 & 72 \\
\hline \multicolumn{9}{|l|}{ APR } \\
\hline $27 \ldots$ & $<.5$ & 77 & 25 & E27 & $<20$ & 14 & 94 & 69 \\
\hline \multicolumn{9}{|l|}{ MAY } \\
\hline $11 \ldots$ & $<.5$ & 97 & 33 & E36 & E8 & 18 & 14 & 73 \\
\hline $27 \ldots$ & $<.5$ & 582 & 47 & 188 & $<20$ & 219 & 299 & 67 \\
\hline \multicolumn{9}{|l|}{ JUN } \\
\hline 10. & $<.5$ & 159 & 23 & E38 & $<20$ & 48 & 65 & 59 \\
\hline $22 .$. & $<.5$ & 132 & 16 & 51 & $<20$ & 53 & 98 & 48 \\
\hline \multicolumn{9}{|l|}{ AUG } \\
\hline $13 \ldots$ & $<.5$ & 147 & 22 & E38 & 5 & 25 & 8.9 & 90 \\
\hline
\end{tabular}

22 Water-quality, bed-sediment, and biological data (October 1998 through September 1999) and statistical summaries of data for streams in the Upper Clark Fork Basin, Montana 
Table 4. Water-quality data for the upper Clark Fork basin, Montana, October 1998 through September 1999 (Continued) 12324590-LITTLE BLACKFOOT RIVER NEAR GARRISON, MONT.

\begin{tabular}{|c|c|c|c|c|c|c|c|c|c|}
\hline Date & Time & $\begin{array}{c}\text { Streamflow, } \\
\text { instan- } \\
\text { taneous } \\
\left(\mathrm{ft}^{3} / \mathrm{s}\right)\end{array}$ & $\begin{array}{c}\text { Specific } \\
\text { conductance, } \\
\text { onsite } \\
(\mu \mathrm{S} / \mathrm{cm})\end{array}$ & $\begin{array}{c}\text { pH, } \\
\text { onsite } \\
\text { (standard } \\
\text { units) }\end{array}$ & $\begin{array}{l}\text { Temper- } \\
\text { ature, } \\
\text { water } \\
\left({ }^{\circ} \mathrm{C}\right)\end{array}$ & $\begin{array}{c}\text { Hardness, } \\
\text { total } \\
(\mathrm{mg} / \mathrm{L} \text { as } \\
\left.\mathrm{CaCO}_{3}\right)\end{array}$ & $\begin{array}{l}\text { Calcium, } \\
\text { dissolved } \\
(\mathbf{m g} / \mathrm{L})\end{array}$ & $\begin{array}{l}\text { Magne- } \\
\text { sium, } \\
\text { dissolved } \\
\text { (mg/L) }\end{array}$ & $\begin{array}{c}\text { Arsenic, } \\
\text { total } \\
\text { recoverable } \\
(\mu \mathrm{g} / \mathrm{L})\end{array}$ \\
\hline \multicolumn{10}{|l|}{ NOV 1998} \\
\hline $17 .$. & 1610 & 83 & 277 & 8.4 & 3.5 & 130 & 36 & 8.5 & 5 \\
\hline \multicolumn{10}{|l|}{ APR 1999} \\
\hline $27 \ldots$ & 1725 & 208 & 206 & 8.4 & 10.5 & 91 & 26 & 6.2 & 5 \\
\hline \multicolumn{10}{|l|}{ MAY } \\
\hline 24 & 1820 & 455 & 168 & 8.2 & 16.5 & 75 & 22 & 5.0 & 8 \\
\hline \multicolumn{10}{|l|}{ JUN } \\
\hline $04 \ldots$ & 1130 & 852 & 186 & 8.0 & 9.5 & 82 & 24 & 5.5 & 9 \\
\hline $23 \ldots$ & 1730 & 208 & 238 & 8.3 & 18.0 & 110 & 31 & 7.2 & 5 \\
\hline \multicolumn{10}{|l|}{ AUG } \\
\hline $10 \ldots$ & 1045 & 54 & 289 & 8.5 & 16.0 & 140 & 39 & 9.0 & 6 \\
\hline
\end{tabular}

\begin{tabular}{|c|c|c|c|c|c|c|c|c|}
\hline Date & $\begin{array}{c}\text { Arsenic, } \\
\text { dissolved } \\
(\mu \mathrm{g} / \mathrm{L})\end{array}$ & $\begin{array}{c}\text { Cadmium, } \\
\text { total } \\
\text { recoverable } \\
(\mu \mathrm{g} / \mathrm{L})\end{array}$ & $\begin{array}{c}\text { Cadmium, } \\
\text { dissolved } \\
(\mu \mathrm{g} / \mathrm{L})\end{array}$ & $\begin{array}{c}\text { Copper, } \\
\text { total } \\
\text { recoverable } \\
(\mu g / L)\end{array}$ & $\begin{array}{c}\text { Copper, } \\
\text { dissolved } \\
(\mu \mathrm{g} / \mathrm{L})\end{array}$ & $\begin{array}{c}\text { Iron, } \\
\text { total } \\
\text { recoverable } \\
(\mu \mathrm{g} / \mathrm{L})\end{array}$ & $\begin{array}{c}\text { Iron, } \\
\text { dissolved } \\
(\mu \mathrm{g} / \mathrm{L})\end{array}$ & $\begin{array}{c}\text { Lead, } \\
\text { total } \\
\text { recoverable } \\
(\mu \mathrm{g} / \mathrm{L})\end{array}$ \\
\hline \multicolumn{9}{|l|}{ NOV 1998} \\
\hline $17 \ldots$ & 3 & $<1$ & $<.1$ & $<1$ & 1 & 60 & $<10$ & $<1$ \\
\hline \multicolumn{9}{|l|}{ APR 1999} \\
\hline $27 \ldots$ & 5 & $<1$ & $<.1$ & 3 & 1 & 367 & 56 & $<1$ \\
\hline \multicolumn{9}{|l|}{ MAY } \\
\hline $24 \ldots$ & 5 & $<1$ & $<.1$ & 5 & 2 & 1,290 & 41 & 3 \\
\hline \multicolumn{9}{|l|}{ JUN } \\
\hline $04 \ldots$ & 6 & $<1$ & $<.1$ & 6 & 3 & 1,950 & 90 & 4 \\
\hline 23. & 6 & $<1$ & $<.1$ & 2 & 1 & 120 & 14 & $<1$ \\
\hline \multicolumn{9}{|l|}{ AUG } \\
\hline $10 \ldots$ & 6 & $<1$ & $<.1$ & 2 & $<1$ & 92 & 13 & $<1$ \\
\hline
\end{tabular}

\begin{tabular}{|c|c|c|c|c|c|c|c|c|}
\hline Date & $\begin{array}{c}\text { Lead, } \\
\text { dissolved } \\
(\mu \mathrm{g} / \mathrm{L})\end{array}$ & $\begin{array}{l}\text { Manga- } \\
\text { nese, } \\
\text { total } \\
\text { recoverable } \\
(\mu \mathrm{g} / \mathrm{L})\end{array}$ & $\begin{array}{c}\text { Manga- } \\
\text { nese, } \\
\text { dissolved } \\
(\mu \mathrm{g} / \mathrm{L})\end{array}$ & $\begin{array}{c}\text { Zinc, } \\
\text { total } \\
\text { recoverable } \\
(\mu \mathrm{g} / \mathrm{L})\end{array}$ & $\begin{array}{c}\text { Zinc, } \\
\text { dissolved } \\
(\mu \mathrm{g} / \mathrm{L})\end{array}$ & $\begin{array}{c}\text { Sediment, } \\
\text { suspended } \\
(\mathrm{mg} / \mathrm{L})\end{array}$ & $\begin{array}{c}\text { Sediment } \\
\text { discharge, } \\
\text { suspended } \\
\text { (ton/d) }\end{array}$ & $\begin{array}{c}\text { Sediment, } \\
\text { suspended } \\
\text { (percent } \\
\text { finer than } \\
0.062 \mathrm{~mm} \text { ) }\end{array}$ \\
\hline \multicolumn{9}{|l|}{ NOV 1998} \\
\hline $17 \ldots$ & $<.5$ & $<10$ & 5 & $<10$ & $<20$ & 3 & .67 & 83 \\
\hline \multicolumn{9}{|l|}{ APR 1999} \\
\hline $27 \ldots$ & $<.5$ & 27 & 7 & $<40$ & $<20$ & 14 & 7.9 & 85 \\
\hline \multicolumn{9}{|l|}{ MAY } \\
\hline $24 \ldots$ & $<.5$ & 78 & 8 & $<40$ & $<20$ & 84 & 103 & 56 \\
\hline \multicolumn{9}{|l|}{ JUN } \\
\hline 04 & $<.5$ & 104 & 10 & $<40$ & $<20$ & 120 & 276 & 51 \\
\hline $23 \ldots$ & $<.5$ & 16 & 10 & $<40$ & $<20$ & 7 & 3.9 & 91 \\
\hline \multicolumn{9}{|l|}{ AUG } \\
\hline $10 \ldots$ & $<.5$ & 21 & 12 & $<40$ & $<1$ & 4 & .58 & 87 \\
\hline
\end{tabular}


Table 4. Water-quality data for the upper Clark Fork basin, Montana, October 1998 through September 1999 (Continued)

12324680-CLARK FORK AT GOLDCREEK, MONT.

\begin{tabular}{|c|c|c|c|c|c|c|c|c|c|}
\hline Date & Time & $\begin{array}{c}\text { Streamflow, } \\
\text { instan- } \\
\text { taneous } \\
\left(\mathrm{ft}^{3} / \mathrm{s}\right)\end{array}$ & $\begin{array}{c}\text { Specific } \\
\text { conductance, } \\
\text { onsite } \\
(\mu \text { S/cm) }\end{array}$ & $\begin{array}{c}\text { pH, } \\
\text { onsite } \\
\text { (standard } \\
\text { units) }\end{array}$ & $\begin{array}{l}\text { Temper- } \\
\text { ature, } \\
\text { water } \\
\left({ }^{\circ} \mathrm{C}\right)\end{array}$ & $\begin{array}{l}\text { Hardness, } \\
\text { total } \\
(\mathrm{mg} / \mathrm{L} \text { as } \\
\left.\mathrm{CaCO}_{3}\right)\end{array}$ & $\begin{array}{l}\text { Calcium, } \\
\text { dissolved } \\
(\mathrm{mg} / \mathrm{L})\end{array}$ & $\begin{array}{l}\text { Magne- } \\
\text { sium, } \\
\text { dissolved } \\
(\mathbf{m g} / \mathrm{L})\end{array}$ & $\begin{array}{c}\text { Arsenic, } \\
\text { total } \\
\text { recoverable } \\
(\mu g / L)\end{array}$ \\
\hline \multicolumn{10}{|l|}{ NOV 1998} \\
\hline 18. & 0810 & 466 & 436 & 8.2 & 2.5 & 190 & 55 & 12 & 10 \\
\hline \multicolumn{10}{|l|}{ FEB 1999} \\
\hline $23 \ldots$ & 0830 & 350 & 458 & 8.5 & 1.5 & 200 & 59 & 13 & 8 \\
\hline \multicolumn{10}{|l|}{ APR } \\
\hline $28 \ldots$ & 0820 & 575 & 369 & 8.2 & 8.5 & 160 & 47 & 11 & 12 \\
\hline \multicolumn{10}{|l|}{ MAY } \\
\hline $11 \ldots$ & 1300 & 620 & 357 & 8.6 & 9.5 & 150 & 44 & 10 & 10 \\
\hline $27 \ldots$ & 1105 & 1,180 & 237 & 8.1 & 12.0 & 100 & 29 & 6.4 & 25 \\
\hline \multicolumn{10}{|l|}{ JUN } \\
\hline $07 \ldots$ & 1455 & 1,720 & 251 & 8.2 & 12.5 & 110 & 31 & 6.7 & 18 \\
\hline $22 \ldots$ & 1800 & 1,080 & 266 & 8.4 & 16.0 & 110 & 33 & 7.2 & 16 \\
\hline \multicolumn{10}{|l|}{ AUG } \\
\hline $13 \ldots$ & 1430 & 248 & 406 & 8.7 & 18.5 & 170 & 51 & 11 & 12 \\
\hline
\end{tabular}

\begin{tabular}{|c|c|c|c|c|c|c|c|c|}
\hline Date & $\begin{array}{c}\text { Arsenic, } \\
\text { dissolved } \\
(\mu \mathrm{g} / \mathrm{L})\end{array}$ & $\begin{array}{c}\text { Cadmium, } \\
\text { total } \\
\text { recoverable } \\
(\mu \mathrm{g} / L)\end{array}$ & $\begin{array}{c}\text { Cadmium, } \\
\text { dissolved } \\
(\mu \mathrm{g} / \mathrm{L})\end{array}$ & $\begin{array}{c}\text { Copper, } \\
\text { total } \\
\text { recoverable } \\
(\mu \mathrm{g} / \mathrm{L})\end{array}$ & $\begin{array}{l}\text { Copper, } \\
\text { dissolved } \\
(\mu \mathrm{g} / \mathrm{L})\end{array}$ & $\begin{array}{c}\text { Iron, } \\
\text { total } \\
\text { recoverable } \\
(\mu \mathrm{g} / \mathrm{L})\end{array}$ & $\begin{array}{c}\text { Iron, } \\
\text { dissolved } \\
(\mu \mathrm{g} / \mathrm{L})\end{array}$ & $\begin{array}{c}\text { Lead, } \\
\text { total } \\
\text { recoverable } \\
(\mu \mathrm{g} / \mathrm{L})\end{array}$ \\
\hline \multicolumn{9}{|l|}{ NOV 1998} \\
\hline $18 \ldots$ & 7 & $<1$ & $<1$ & 17 & 3 & 270 & $<10$ & 2 \\
\hline \multicolumn{9}{|l|}{ FEB 1999} \\
\hline 23 & 6 & $<1$ & $<.1$ & 16 & 4 & 230 & $<10$ & 1 \\
\hline \multicolumn{9}{|l|}{ APR } \\
\hline 28 & 7 & $<1$ & .1 & 35 & 4 & 725 & 13 & 4 \\
\hline \multicolumn{9}{|l|}{ MAY } \\
\hline $11 \ldots$ & 8 & $<1$ & $<.1$ & 19 & 4 & 426 & E8 & 2 \\
\hline $27 \ldots$ & 13 & $<1$ & $<.1$ & 100 & 6 & 2,240 & 33 & 15 \\
\hline \multicolumn{9}{|l|}{ JUN } \\
\hline 07. & 11 & $<1$ & $<.1$ & 48 & 8 & 1,140 & 29 & 7 \\
\hline $22 \ldots$ & 12 & $<1$ & $<.1$ & 47 & 8 & 725 & 15 & 5 \\
\hline \multicolumn{9}{|l|}{ AUG } \\
\hline $13 \ldots$ & 11 & $<1$ & $<.1$ & 16 & 6 & 209 & E5 & 1 \\
\hline
\end{tabular}

\begin{tabular}{|c|c|c|c|c|c|c|c|c|}
\hline Date & $\begin{array}{c}\text { Lead, } \\
\text { dissolved } \\
(\mu \mathrm{g} / \mathrm{L})\end{array}$ & $\begin{array}{c}\text { Manga- } \\
\text { nese, } \\
\text { total } \\
\text { recoverable } \\
(\mu \mathrm{g} / \mathrm{L})\end{array}$ & $\begin{array}{l}\text { Manga- } \\
\text { nese, } \\
\text { dissolved } \\
(\mu \mathrm{g} / \mathrm{L})\end{array}$ & $\begin{array}{c}\text { Zinc, } \\
\text { total } \\
\text { recoverable } \\
(\mu \mathrm{g} / \mathrm{L})\end{array}$ & $\begin{array}{c}\text { Zinc, } \\
\text { dissolved } \\
(\mu \mathrm{g} / \mathrm{L})\end{array}$ & $\begin{array}{l}\text { Sediment, } \\
\text { suspended } \\
\text { (mg/L) }\end{array}$ & $\begin{array}{l}\text { Sediment } \\
\text { discharge, } \\
\text { suspended } \\
\text { (ton/d) }\end{array}$ & $\begin{array}{l}\text { Sediment, } \\
\text { suspended } \\
\text { (percent } \\
\text { finer than } \\
0.062 \mathrm{~mm} \text { ) }\end{array}$ \\
\hline \multicolumn{9}{|l|}{ NOV 1998} \\
\hline $18 \ldots$ & $<.5$ & 70 & 16 & 20 & $<20$ & 12 & 15 & 81 \\
\hline \multicolumn{9}{|l|}{ FEB 1999} \\
\hline $23 \ldots$ & $<.5$ & 70 & 33 & 20 & E13 & 11 & 10 & 83 \\
\hline \multicolumn{9}{|l|}{ APR } \\
\hline $28 \ldots$ & $<.5$ & 111 & 21 & $<40$ & $<20$ & 38 & 59 & 83 \\
\hline \multicolumn{9}{|l|}{ MAY } \\
\hline $11 \ldots$ & $<.5$ & 68 & 12 & E23 & $<20$ & 22 & 37 & 81 \\
\hline $27 \ldots$ & $<.5$ & 299 & 12 & 102 & $<20$ & 126 & 401 & 75 \\
\hline \multicolumn{9}{|l|}{ JUN } \\
\hline $07 .$. & $<5$ & 129 & 12 & 44 & $<20$ & 72 & 334 & 51 \\
\hline $22 \ldots$ & $<.5$ & 109 & 10 & 48 & $<20$ & 41 & 120 & 60 \\
\hline \multicolumn{9}{|l|}{ AUG } \\
\hline $13 \ldots$ & $<.5$ & 78 & 10 & E21 & 2 & 11 & 74 & 86 \\
\hline
\end{tabular}

24 Water-quality, bed-sediment, and biological data (October 1998 through September 1999) and statistical summaries of data for streams in the Upper Clark Fork Basin, Montana 
Table 4. Water-quality data for the upper Clark Fork basin, Montana, October 1998 through September 1999 (Continued)

\begin{tabular}{|c|c|c|c|c|c|c|c|c|c|}
\hline Date & Time & $\begin{array}{c}\text { Streamflow, } \\
\text { instan- } \\
\text { taneous } \\
\left(\mathrm{ft}^{3} / \mathrm{s}\right)\end{array}$ & $\begin{array}{c}\text { Specific } \\
\text { conductance, } \\
\text { onsite } \\
(\mu \mathrm{S} / \mathbf{c m})\end{array}$ & $\begin{array}{c}\mathrm{pH}, \\
\text { onsite } \\
\text { (standard } \\
\text { units) }\end{array}$ & $\begin{array}{l}\text { Temper- } \\
\text { ature, } \\
\text { water } \\
\left({ }^{\circ} \mathrm{C}\right)\end{array}$ & $\begin{array}{c}\text { Hardness, } \\
\text { total } \\
\left(\mathrm{mg}^{\prime} \mathrm{L} \text { as }\right. \\
\left.\mathrm{CaCO}_{3}\right)\end{array}$ & $\begin{array}{c}\text { Calcium, } \\
\text { dissolved } \\
(\mathrm{mg} / \mathrm{L})\end{array}$ & $\begin{array}{l}\text { Magne- } \\
\text { sium, } \\
\text { dissolved } \\
(\mathrm{mg} / \mathrm{L})\end{array}$ & $\begin{array}{c}\text { Arsenic, } \\
\text { total } \\
\text { recoverable } \\
(\mu \mathrm{g} / \mathrm{L})\end{array}$ \\
\hline \multicolumn{10}{|l|}{ NOV 1998} \\
\hline $18 \ldots$ & 0915 & 174 & 324 & 8.4 & 3.0 & 150 & 39 & 12 & 8 \\
\hline \multicolumn{10}{|l|}{ FEB 1999} \\
\hline $23 \ldots$ & 0940 & E120 & 307 & 8.6 & 1.5 & 140 & 39 & 12 & 8 \\
\hline \multicolumn{10}{|l|}{ APR } \\
\hline $28 \ldots$ & 0930 & E240 & 214 & 8.3 & 7.0 & 96 & 25 & 8.0 & 51 \\
\hline \multicolumn{10}{|l|}{ MAY } \\
\hline $11 .$. & 1405 & 156 & 251 & 8.7 & 10.0 & 110 & 30 & 9.2 & 13 \\
\hline $27 \ldots$ & 0955 & 253 & 146 & 8.3 & 8.0 & 65 & 18 & 4.8 & 19 \\
\hline \multicolumn{10}{|l|}{ JUN } \\
\hline $07 \ldots$ & 1540 & 324 & 206 & 8.2 & 11.0 & 96 & 26 & 7.3 & 20 \\
\hline $23 \ldots$ & 1620 & 172 & 265 & 8.6 & 17.0 & 120 & 33 & 9.1 & 13 \\
\hline \multicolumn{10}{|l|}{ AUG } \\
\hline $10 \ldots$ & 1610 & 35 & 434 & 8.8 & 20.5 & 200 & 55 & 16 & 12 \\
\hline
\end{tabular}

\begin{tabular}{|c|c|c|c|c|c|c|c|c|}
\hline Date & $\begin{array}{c}\text { Arsenic, } \\
\text { dissolved } \\
(\mu \mathrm{g} / \mathrm{L})\end{array}$ & $\begin{array}{c}\text { Cadmium, } \\
\text { total } \\
\text { recoverable } \\
(\mu \mathrm{g} / \mathrm{L})\end{array}$ & $\begin{array}{c}\text { Cadmium, } \\
\text { dissolved } \\
(\mu \mathrm{g} / \mathrm{L})\end{array}$ & $\begin{array}{c}\text { Copper, } \\
\text { total } \\
\text { recoverable } \\
(\mu \mathrm{g} / \mathrm{L})\end{array}$ & $\begin{array}{l}\text { Copper, } \\
\text { dissolved } \\
(\mu \mathrm{g} / \mathrm{L})\end{array}$ & $\begin{array}{l}\text { Iron, } \\
\text { total } \\
\text { recoverable } \\
(\mu \mathrm{g} / \mathrm{L})\end{array}$ & $\begin{array}{c}\text { Iron, } \\
\text { dissolved } \\
(\mu \mathrm{g} / \mathrm{L})\end{array}$ & $\begin{array}{c}\text { Lead, } \\
\text { total } \\
\text { recoverable } \\
(\mu \mathrm{g} / \mathrm{L})\end{array}$ \\
\hline \multicolumn{9}{|l|}{ NOV 1998} \\
\hline 18 & 5 & $<1$ & $<1$ & 1 & 1 & 160 & $<10$ & 2 \\
\hline \multicolumn{9}{|l|}{ FEB 1999} \\
\hline $23 \ldots$ & 6 & $<1$ & $<.1$ & 2 & $<1$ & 180 & E8 & 2 \\
\hline \multicolumn{9}{|l|}{ APR } \\
\hline $28 \ldots$ & 9 & $<1$ & .1 & 14 & 3 & 1,970 & 91 & 32 \\
\hline \multicolumn{9}{|l|}{ MAY } \\
\hline $11 \ldots$ & 7 & $<1$ & $<1$ & 6 & 2 & 565 & 51 & 8 \\
\hline $27 \ldots$ & 7 & $<1$ & $<1$ & 7 & 2 & 803 & 52 & 14 \\
\hline \multicolumn{9}{|l|}{ JUN } \\
\hline $07 \ldots$ & 7 & $<1$ & $<1$ & 5 & 2 & 685 & 27 & 14 \\
\hline $23 \ldots$ & 8 & $<1$ & $<1$ & 4 & 2 & 297 & 42 & 5 \\
\hline \multicolumn{9}{|l|}{ AUG } \\
\hline $10 \ldots$ & 11 & $<1$ & $<1$ & 3 & 1 & 133 & E10 & 1 \\
\hline
\end{tabular}

\begin{tabular}{|c|c|c|c|c|c|c|c|c|}
\hline Date & $\begin{array}{l}\text { Lead, } \\
\text { dissolved } \\
(\mu \mathrm{g} / \mathrm{L})\end{array}$ & $\begin{array}{l}\text { Manga- } \\
\text { nese, } \\
\text { total } \\
\text { recoverable } \\
(\mu \mathrm{g} / \mathrm{L})\end{array}$ & $\begin{array}{c}\text { Manga- } \\
\text { nese, } \\
\text { dissolved } \\
(\mu \mathrm{g} / \mathrm{L})\end{array}$ & $\begin{array}{c}\text { Zinc, } \\
\text { total } \\
\text { recoverable } \\
(\mu \mathrm{g} / \mathrm{L})\end{array}$ & $\begin{array}{c}\text { Zinc, } \\
\text { dissolved } \\
(\mu \mathrm{g} / \mathrm{L})\end{array}$ & $\begin{array}{l}\text { Sediment, } \\
\text { suspended } \\
(\mathrm{mg} / \mathrm{L})\end{array}$ & $\begin{array}{l}\text { Sediment } \\
\text { discharge, } \\
\text { suspended } \\
\text { (ton/d) }\end{array}$ & $\begin{array}{l}\text { Sediment, } \\
\text { suspended } \\
\text { (percent } \\
\text { finer than } \\
0.062 \mathrm{~mm} \text { ) }\end{array}$ \\
\hline \multicolumn{9}{|l|}{ NOV 1998} \\
\hline $18 \ldots$ & $<.5$ & 60 & 20 & $<10$ & $<20$ & 8 & 3.8 & 86 \\
\hline \multicolumn{9}{|l|}{ FEB 1999} \\
\hline $23 \ldots$ & $<.5$ & 60 & 15 & $<10$ & $<20$ & 8 & 2.6 & 90 \\
\hline \multicolumn{9}{|l|}{ APR } \\
\hline $28 \ldots$ & .7 & 405 & 29 & 74 & $<20$ & 133 & 86 & 86 \\
\hline \multicolumn{9}{|l|}{ MAY } \\
\hline 11. & .6 & 124 & 23 & E25 & $<20$ & 29 & 12 & 90 \\
\hline $27 \ldots$ & .5 & 192 & 19 & 40 & $<20$ & 51 & 35 & 80 \\
\hline \multicolumn{9}{|l|}{ JUN } \\
\hline 07. & .5 & 210 & 19 & E34 & $<20$ & 55 & 48 & 59 \\
\hline $23 \ldots$ & $<.5$ & 104 & 28 & $<40$ & E8 & 19 & 88 & 74 \\
\hline \multicolumn{9}{|l|}{ AUG } \\
\hline $10 \ldots$ & $<.5$ & 88 & 53 & $<40$ & 4 & 7 & .66 & 89 \\
\hline
\end{tabular}


Table 4. Water-quality data for the upper Clark Fork basin. Montana. October 1998 through September 1999 (Continued)

12331800--CLARK FORK NEAR DRUMMOND, MONT.

\begin{tabular}{|c|c|c|c|c|c|c|c|c|c|}
\hline Date & Time & $\begin{array}{c}\text { Streamflow, } \\
\text { instan- } \\
\text { taneous } \\
\left(\mathrm{ft}^{3} / \mathrm{s}\right)\end{array}$ & $\begin{array}{c}\text { Specific } \\
\text { conductance, } \\
\text { onsite } \\
(\mu \mathrm{S} / \mathrm{cm})\end{array}$ & $\begin{array}{c}\text { pH, } \\
\text { onsite } \\
\text { (standard } \\
\text { units) }\end{array}$ & $\begin{array}{l}\text { Temper- } \\
\text { ature, } \\
\text { water } \\
\left({ }^{\circ} \mathrm{C}\right)\end{array}$ & $\begin{array}{c}\text { Hardness, } \\
\text { total } \\
(\mathrm{mg} / \mathrm{L} \text { as } \\
\left.\mathrm{CaCO})_{3}\right)\end{array}$ & $\begin{array}{l}\text { Calcium, } \\
\text { dissolved } \\
\text { (mg/L) }\end{array}$ & $\begin{array}{l}\text { Magne- } \\
\text { sium, } \\
\text { dissolved } \\
(\mathrm{mg} / \mathrm{L})\end{array}$ & $\begin{array}{c}\text { Arsenic, } \\
\text { total } \\
\text { recoverable } \\
(\mu \mathrm{g} / \mathrm{L})\end{array}$ \\
\hline \multicolumn{10}{|l|}{ NOV 1998} \\
\hline 18 & 1020 & 755 & 454 & 8.3 & 4.5 & 200 & 56 & 14 & 10 \\
\hline \multicolumn{10}{|l|}{ FEB 1999} \\
\hline $24 \ldots$ & 0945 & 542 & 463 & 8.4 & 3.5 & 210 & 61 & 15 & 8 \\
\hline \multicolumn{10}{|l|}{ APR } \\
\hline $28 \ldots$ & 1030 & 883 & 363 & 8.2 & 9.5 & 160 & 45 & 11 & 20 \\
\hline \multicolumn{10}{|l|}{ MAY } \\
\hline 11. & 1505 & 842 & 384 & 8.5 & 11.0 & 170 & 49 & 12 & 11 \\
\hline $27 \ldots$ & 0810 & 1,340 & 281 & 8.1 & 12.5 & 120 & 34 & 8.1 & 28 \\
\hline \multicolumn{10}{|l|}{ JUN } \\
\hline $07 \ldots$ & 1415 & 2,330 & 276 & 8.2 & 11.5 & 120 & 35 & 8.0 & 22 \\
\hline $23 \ldots$ & 1500 & 1,390 & 320 & 8.4 & 17.0 & 140 & 41 & 94 & 15 \\
\hline \multicolumn{10}{|l|}{ AUG } \\
\hline $13 \ldots$ & 1615 & 412 & 513 & 8.5 & 18.0 & 240 & 67 & 17 & 13 \\
\hline
\end{tabular}

\begin{tabular}{|c|c|c|c|c|c|c|c|c|}
\hline Date & $\begin{array}{c}\text { Arsenic, } \\
\text { dissolved } \\
(\mu \mathrm{g} / \mathrm{L})\end{array}$ & $\begin{array}{c}\text { Cadmium. } \\
\text { total } \\
\text { recoverable } \\
(\mu \mathrm{g} / \mathrm{L})\end{array}$ & $\begin{array}{c}\text { Cadmium, } \\
\text { dissolved } \\
(\mu \mathrm{g} / \mathrm{L})\end{array}$ & $\begin{array}{c}\text { Copper, } \\
\text { total } \\
\text { recoverable } \\
(\mu \mathrm{g} / \mathrm{L})\end{array}$ & $\begin{array}{l}\text { Copper, } \\
\text { dissolved } \\
(\mu \mathrm{g} / \mathrm{L})\end{array}$ & $\begin{array}{c}\text { Iron, } \\
\text { total } \\
\text { recoverable } \\
(\mu \mathrm{g} / \mathrm{L})\end{array}$ & $\begin{array}{c}\text { Iron, } \\
\text { dissolved } \\
(\mu \mathrm{g} / \mathrm{L})\end{array}$ & $\begin{array}{c}\text { Lead, } \\
\text { total } \\
\text { recoverable } \\
(\mu \mathrm{g} / \mathrm{L})\end{array}$ \\
\hline \multicolumn{9}{|l|}{ NOV 1998} \\
\hline $18 \ldots$ & 7 & $<1$ & $<.1$ & 13 & 3 & 220 & $<10$ & 2 \\
\hline \multicolumn{9}{|l|}{ FEB 1999} \\
\hline $24 \ldots$ & 7 & $<1$ & $<.1$ & 12 & 4 & 200 & E5 & 2 \\
\hline \multicolumn{9}{|l|}{ APR } \\
\hline $28 \ldots$ & 8 & $<1$ & $<1$ & 34 & 4 & 1,040 & 20 & 11 \\
\hline \multicolumn{9}{|l|}{ MAY } \\
\hline $11 \ldots$ & 10 & $<1$ & $<.1$ & 23 & 5 & 515 & E8 & 3 \\
\hline $27 \ldots$ & 13 & $<1$ & $<1$ & 100 & 6 & 2,360 & 30 & 18 \\
\hline \multicolumn{9}{|l|}{ JUN } \\
\hline $07 \ldots$ & 11 & $<1$ & $<.1$ & 71 & 9 & 1,790 & 26 & 13 \\
\hline $23 \ldots$ & 14 & $<1$ & $<1$ & 37 & 8 & 695 & 11 & 4 \\
\hline \multicolumn{9}{|l|}{ AUG } \\
\hline $13 \ldots$ & 13 & $<1$ & $<.1$ & 19 & 5 & 373 & $<10$ & 3 \\
\hline
\end{tabular}

\begin{tabular}{|c|c|c|c|c|c|c|c|c|}
\hline Date & $\begin{array}{c}\text { Lead, } \\
\text { dissolved } \\
(\mu \mathrm{g} / \mathrm{L})\end{array}$ & $\begin{array}{l}\text { Manga- } \\
\text { nese, } \\
\text { total } \\
\text { recoverable } \\
(\mu \mathrm{g} / \mathrm{L})\end{array}$ & $\begin{array}{l}\text { Manga- } \\
\text { nese, } \\
\text { dissolved } \\
(\mu \mathrm{g} / \mathrm{L})\end{array}$ & $\begin{array}{c}\text { Zinc, } \\
\text { total } \\
\text { recoverable } \\
(\mu \mathrm{g} / \mathrm{L})\end{array}$ & $\begin{array}{c}\text { Zinc, } \\
\text { dissolved } \\
(\mu \mathrm{g} / \mathrm{L})\end{array}$ & $\begin{array}{l}\text { Sediment, } \\
\text { suspended } \\
\text { (mg } / \mathrm{L})\end{array}$ & $\begin{array}{c}\text { Sediment } \\
\text { discharge, } \\
\text { suspended } \\
\text { (ton/d) }\end{array}$ & $\begin{array}{l}\text { Sediment, } \\
\text { suspended } \\
\text { (percent } \\
\text { finer than } \\
0.062 \mathrm{~mm} \text { ) }\end{array}$ \\
\hline \multicolumn{9}{|l|}{ NOV 1998} \\
\hline $18 \ldots$ & $<.5$ & 70 & 10 & 20 & $<20$ & 11 & 22 & 87 \\
\hline \multicolumn{9}{|l|}{ FEB 1999} \\
\hline 24 & $<.5$ & 50 & 20 & 20 & $<20$ & 13 & 19 & 73 \\
\hline \multicolumn{9}{|l|}{ APR } \\
\hline $28 \ldots$ & $<.5$ & 172 & 13 & 43 & $<20$ & 63 & 150 & 80 \\
\hline \multicolumn{9}{|l|}{ MAY } \\
\hline $11 \ldots$ & $<.5$ & 82 & 13 & E25 & $<20$ & 29 & 66 & 78 \\
\hline $27 \ldots$ & $<.5$ & 337 & 10 & 142 & $<20$ & 143 & 517 & 82 \\
\hline \multicolumn{9}{|l|}{ JUN } \\
\hline $07 \ldots$ & $<.5$ & 219 & 12 & 96 & E8 & 122 & 768 & 56 \\
\hline $23 \ldots$ & $<.5$ & 96 & 11 & 44 & $<20$ & 38 & 143 & 63 \\
\hline \multicolumn{9}{|l|}{ AUG } \\
\hline $13 \ldots$ & $<.5$ & 128 & 8 & E34 & 3 & 25 & 28 & 89 \\
\hline
\end{tabular}


Table 4. Water-quality data for the upper Clark Fork basin, Montana, October 1998 through September 1999 (Continued) 12334510--ROCK CREEK NEAR CLINTON, MONT.

\begin{tabular}{|c|c|c|c|c|c|c|c|c|c|}
\hline Date & Time & $\begin{array}{l}\text { Streamflow, } \\
\text { instan- } \\
\text { taneous } \\
\left(\mathrm{ft}^{3} / \mathrm{s}\right)\end{array}$ & $\begin{array}{c}\text { Specific } \\
\text { conductance, } \\
\text { onsite } \\
(\mu \mathrm{S} / \mathrm{cm})\end{array}$ & $\begin{array}{c}\mathrm{pH}, \\
\text { onsite } \\
\text { (standard } \\
\text { units) }\end{array}$ & $\begin{array}{c}\text { Temper- } \\
\text { ature, } \\
\text { water } \\
\left.{ }^{\circ} \mathrm{C}\right)\end{array}$ & $\begin{array}{c}\text { Hardness, } \\
\text { total } \\
(\mathrm{mg} / \mathrm{L} \text { as } \\
\left.\mathrm{CaCO}_{3}\right)\end{array}$ & $\begin{array}{c}\text { Calcium, } \\
\text { dissolved } \\
(\mathrm{mg} / \mathrm{L})\end{array}$ & $\begin{array}{l}\text { Magne- } \\
\text { sium, } \\
\text { dissolved } \\
(\mathrm{mg} / \mathrm{L})\end{array}$ & $\begin{array}{c}\text { Arsenic, } \\
\text { total } \\
\text { recoverable } \\
(\mu g / L)\end{array}$ \\
\hline \multicolumn{10}{|l|}{ NOV 1998} \\
\hline 18 & 1145 & 252 & 142 & 8.5 & 4.0 & 63 & 16 & 5.6 & $<1$ \\
\hline \multicolumn{10}{|l|}{ APR 1999} \\
\hline $28 \ldots$ & 1245 & 1,000 & 85 & 8.2 & 6.0 & 37 & 9.4 & 3.3 & $<1$ \\
\hline \multicolumn{10}{|l|}{ MAY } \\
\hline $26 \ldots$ & 1045 & 3,040 & 53 & 7.8 & 9.0 & 23 & 6.0 & 1.9 & 1 \\
\hline \multicolumn{10}{|l|}{ JUN } \\
\hline $09 \ldots$ & 1320 & 2,190 & 68 & 7.8 & 7.0 & 30 & 7.8 & 2.5 & $<1$ \\
\hline $23 \ldots$ & 1310 & 1,730 & $8 I$ & 8.0 & 12.0 & 37 & 9.7 & 3.0 & $<1$ \\
\hline \multicolumn{10}{|l|}{ AUG } \\
\hline $13 \ldots$ & 1720 & 358 & 133 & 8.8 & 15.5 & 61 & 16 & 5.1 & $<1$ \\
\hline
\end{tabular}

\begin{tabular}{|c|c|c|c|c|c|c|c|c|}
\hline Date & $\begin{array}{l}\text { Arsenic, } \\
\text { dissolved } \\
(\mu \mathrm{g} / \mathrm{L})\end{array}$ & $\begin{array}{c}\text { Cadmium, } \\
\text { total } \\
\text { recoverable } \\
(\mu \mathrm{g} / \mathrm{L})\end{array}$ & $\begin{array}{c}\text { Cadmium, } \\
\text { dissolved } \\
(\mu g / L)\end{array}$ & $\begin{array}{c}\text { Copper, } \\
\text { total } \\
\text { recoverable } \\
(\mu \mathrm{g} / \mathrm{L})\end{array}$ & $\begin{array}{c}\text { Copper, } \\
\text { dissolved } \\
(\mu \mathrm{g} / \mathrm{L})\end{array}$ & $\begin{array}{c}\text { Iron, } \\
\text { total } \\
\text { recoverable } \\
(\mu \mathrm{g} / \mathrm{L})\end{array}$ & $\begin{array}{l}\text { Iron, } \\
\text { dissolved } \\
(\mu \mathrm{g} / \mathrm{L})\end{array}$ & $\begin{array}{c}\text { Lead, } \\
\text { total } \\
\text { recoverable } \\
(\mu \mathrm{g} / \mathrm{L})\end{array}$ \\
\hline \multicolumn{9}{|l|}{ NOV 1998} \\
\hline $18 \ldots$ & $<1$ & $<1$ & $<.1$ & $<1$ & $<1$ & 30 & E6 & $<1$ \\
\hline \multicolumn{9}{|l|}{ APR 1999} \\
\hline $28 \ldots$ & $<1$ & $<1$ & $<.1$ & 3 & 2 & 389 & 94 & $<1$ \\
\hline \multicolumn{9}{|l|}{ MAY } \\
\hline $26 \ldots$ & $<1$ & $<1$ & $<.1$ & 3 & $<1$ & 1,340 & 74 & 1 \\
\hline \multicolumn{9}{|l|}{ JUN } \\
\hline $09 \ldots$ & $<1$ & $<1$ & $<1$ & 2 & 1 & 189 & 48 & $<1$ \\
\hline 23. & $<1$ & $<1$ & $<.1$ & 1 & $<1$ & 147 & 33 & $<1$ \\
\hline \multicolumn{9}{|l|}{ AUG } \\
\hline $13 \ldots$ & $<1$ & $<1$ & $<.1$ & $<1$ & $<1$ & 35 & 11 & $<1$ \\
\hline
\end{tabular}

\begin{tabular}{|c|c|c|c|c|c|c|c|c|}
\hline Date & $\begin{array}{l}\text { Lead, } \\
\text { dissolved } \\
(\mu \mathrm{g} / \mathrm{L})\end{array}$ & $\begin{array}{c}\text { Manga- } \\
\text { nese, } \\
\text { total } \\
\text { recoverable } \\
(\mu \mathrm{g} / \mathrm{L})\end{array}$ & $\begin{array}{l}\text { Manga- } \\
\text { nese, } \\
\text { dissolved } \\
(\mu \mathrm{g} / \mathrm{L})\end{array}$ & $\begin{array}{c}\text { Zinc, } \\
\text { total } \\
\text { recoverable } \\
(\mu \mathrm{g} / \mathrm{L})\end{array}$ & $\begin{array}{l}\text { Zinc, } \\
\text { dissolved } \\
(\mu \mathrm{g} / \mathrm{L})\end{array}$ & $\begin{array}{l}\text { Sediment, } \\
\text { snspended } \\
\text { (mg/L) }\end{array}$ & $\begin{array}{c}\text { Sediment } \\
\text { discharge, } \\
\text { suspended } \\
\text { (ton/d) }\end{array}$ & $\begin{array}{c}\text { Sediment, } \\
\text { suspended } \\
\text { (percent } \\
\text { finer than } \\
0.062 \mathrm{~mm} \text { ) }\end{array}$ \\
\hline \multicolumn{9}{|l|}{ NOV 1998} \\
\hline 18 & $<.5$ & $<10$ & $<4$ & $<10$ & $<20$ & 2 & 14 & 62 \\
\hline \multicolumn{9}{|l|}{ APR 1999} \\
\hline 28 & $<5$ & 16 & E2 & $<40$ & $<20$ & 20 & 54 & 71 \\
\hline \multicolumn{9}{|l|}{ MAY } \\
\hline 26. & $<.5$ & 64 & 4 & $<40$ & $<20$ & 205 & 1,680 & 35 \\
\hline \multicolumn{9}{|l|}{ JUN } \\
\hline 09 & $<.5$ & 9 & E3 & $<40$ & E7 & 14 & 83 & 55 \\
\hline 23. & $<.5$ & 7 & E3 & $<40$ & $<20$ & 9 & 42 & 69 \\
\hline \multicolumn{9}{|l|}{ AUG } \\
\hline $13 \ldots$ & $<.5$ & 3 & 1 & $<40$ & 2 & 2 & 1.9 & 70 \\
\hline
\end{tabular}


Table 4. Water-quality data for the upper Clark Fork basin, Montana, October 1998 through September 1999 (Continued)

12334550--CLARK FORK AT TURAH BRIDGE, NEAR BONNER, MONT.

\begin{tabular}{|c|c|c|c|c|c|c|c|c|c|}
\hline Date & Time & $\begin{array}{c}\text { Streamflow, } \\
\text { instan- } \\
\text { taneous } \\
\left(\mathrm{ft}^{3} / \mathrm{s}\right)\end{array}$ & $\begin{array}{c}\text { Specific } \\
\text { conductance, } \\
\text { onsite } \\
(\mu \mathrm{S} / \mathrm{cm})\end{array}$ & $\begin{array}{c}\text { pH, } \\
\text { onsite } \\
\text { (standard } \\
\text { units) }\end{array}$ & $\begin{array}{l}\text { Temper- } \\
\text { ature, } \\
\text { water } \\
\left({ }^{\circ} \mathrm{C}\right)\end{array}$ & $\begin{array}{c}\text { Hardness, } \\
\text { total } \\
(\mathrm{mg} / \mathrm{L} \text { as } \\
\left.\mathrm{CaCO}_{3}\right)\end{array}$ & $\begin{array}{c}\text { Calcium, } \\
\text { dissolved } \\
(\mathrm{mg} / \mathrm{L})\end{array}$ & $\begin{array}{l}\text { Magne- } \\
\text { sium, } \\
\text { dissolved } \\
(\mathrm{mg} / \mathrm{L})\end{array}$ & $\begin{array}{c}\text { Arsenic, } \\
\text { total } \\
\text { recoverable } \\
(\mu \mathrm{g} / \mathrm{L})\end{array}$ \\
\hline \multicolumn{10}{|l|}{ NOV 1998} \\
\hline $18 \ldots$ & 1350 & 1,070 & 373 & 86 & 5.0 & 160 & 46 & 12 & 7 \\
\hline \multicolumn{10}{|l|}{ FEB 1999} \\
\hline $24 \ldots$ & 1120 & 820 & 380 & 8.6 & 4.0 & 170 & 48 & 12 & 6 \\
\hline \multicolumn{10}{|l|}{ APR } \\
\hline $28 \ldots$ & 1445 & 2,100 & 227 & 8.2 & 8.0 & 100 & 28 & 7.6 & 8 \\
\hline \multicolumn{10}{|l|}{ MAY } \\
\hline $12 \ldots$ & 0905 & 1,780 & 268 & 8.4 & 8.5 & 120 & 32 & 8.6 & 6 \\
\hline 125. & 1025 & 3,360 & 160 & 79 & 13.0 & 68 & 19 & 4.9 & 10 \\
\hline$I_{26}$. & 0900 & 3,940 & 143 & 7.8 & 12.0 & 62 & 17 & 4.5 & 10 \\
\hline $26 \ldots$ & 1250 & 4,030 & 141 & 8.1 & 12.0 & 59 & 16 & 4.3 & 11 \\
\hline${ }^{1} 28$ & 0825 & 4,330 & 138 & 7.8 & 11.0 & 60 & 17 & 4.4 & 9 \\
\hline \multicolumn{10}{|l|}{ JUN } \\
\hline${ }^{\prime} 02 \ldots$ & 1100 & 4,630 & 163 & 8.1 & 11.0 & 70 & 20 & 5.0 & 11 \\
\hline '04... & 1020 & 6,410 & 153 & 7.7 & 10.0 & 65 & 19 & 4.5 & 16 \\
\hline $\mathbf{I}^{0} 07 \ldots$ & 0900 & 5,360 & 164 & 7.8 & 9.0 & 69 & 20 & 4.9 & 10 \\
\hline $07 \ldots$ & 1210 & 5,310 & 165 & 8.1 & 9.5 & 70 & 20 & 5.0 & 12 \\
\hline${ }^{1} 09 \ldots$ & 0905 & 4,560 & 184 & 8.0 & 8.0 & 82 & 23 & 5.9 & 9 \\
\hline $23 \ldots$ & 1120 & 3,380 & 192 & 8.3 & 13.0 & 84 & 24 & 60 & 7 \\
\hline \multicolumn{10}{|l|}{ AUG } \\
\hline $10 \ldots$ & 1330 & 648 & 329 & 8.8 & 19.5 & 140 & 40 & 11 & 6 \\
\hline${ }^{1} 15$ & 1220 & 831 & 346 & 8.5 & 15.0 & 160 & 44 & 12 & 8 \\
\hline $1_{19} .$. & 1030 & 730 & 360 & 8.4 & 18.0 & 160 & 45 & 12 & 8 \\
\hline $\mathrm{I}_{22}$ & 0800 & 639 & 360 & 8.3 & 15.0 & 160 & 45 & 12 & 8 \\
\hline $125 \ldots$ & 0945 & 596 & 360 & 8.4 & 15.0 & 160 & 45 & 12 & 7 \\
\hline
\end{tabular}


Table 4. Water-quality data for the upper Clark Fork basin, Montana, October 1998 through September 1999 (Continued) 12334550--CLARK FORK AT TURAH BRIDGE, NEAR BONNER, MONT. (Continued)

\begin{tabular}{|c|c|c|c|c|c|c|c|c|}
\hline Date & $\begin{array}{c}\text { Arsenic, } \\
\text { dissolved } \\
(\mu \mathrm{g} / \mathrm{L})\end{array}$ & $\begin{array}{c}\text { Cadmium, } \\
\text { total } \\
\text { recoverable } \\
(\mu \mathrm{g} / \mathrm{L})\end{array}$ & $\begin{array}{c}\text { Cadmium, } \\
\text { dissolved } \\
(\mu \mathrm{g} / \mathrm{L})\end{array}$ & $\begin{array}{c}\text { Copper, } \\
\text { total } \\
\text { recoverable } \\
(\mu \mathrm{g} / \mathrm{L})\end{array}$ & $\begin{array}{c}\text { Copper, } \\
\text { dissolved } \\
(\mu \mathrm{g} / \mathrm{L})\end{array}$ & $\begin{array}{c}\text { Iron, } \\
\text { total } \\
\text { recoverable } \\
(\mu \mathrm{g} / \mathrm{L})\end{array}$ & $\begin{array}{c}\text { Iron, } \\
\text { dissolved } \\
(\mu \mathrm{g} / \mathrm{L})\end{array}$ & $\begin{array}{c}\text { Lead, } \\
\text { total } \\
\text { recoverable } \\
(\mu \mathrm{g} / \mathrm{L})\end{array}$ \\
\hline \multicolumn{9}{|l|}{ NOV 1998} \\
\hline $18 \ldots$ & 5 & $<1$ & $<.1$ & 8 & 3 & 150 & $<10$ & 1 \\
\hline \multicolumn{9}{|l|}{ FEB 1999} \\
\hline $24 \ldots$ & 5 & $<1$ & $<.1$ & 7 & 3 & 100 & $<10$ & $<1$ \\
\hline \multicolumn{9}{|l|}{ APR } \\
\hline $28 \ldots$ & 5 & $<1$ & $<, 1$ & 22 & 4 & 773 & 49 & 5 \\
\hline \multicolumn{9}{|l|}{ MAY } \\
\hline $12 \ldots$ & 5 & $<1$ & $<.1$ & 13 & 3 & 313 & 16 & 2 \\
\hline${ }^{1} 25 \ldots$ & 4 & $<1$ & $<.1$ & 57 & 4 & 2,010 & 32 & 9 \\
\hline${ }^{\mathbf{I}} 26 \ldots$ & 5 & $<1$ & $<.1$ & 61 & 5 & 2,680 & 45 & 12 \\
\hline $26 \ldots$ & 5 & $<1$ & $<.1$ & 60 & 4 & 2,010 & 51 & 10 \\
\hline${ }^{\prime} 28 \ldots$ & 5 & $<1$ & $<.1$ & 46 & 5 & 1,720 & 46 & 8 \\
\hline \multicolumn{9}{|l|}{$\pi N$} \\
\hline${ }^{1} 02 \ldots$ & 6 & $<1$ & $<.1$ & 59 & 6 & 1,540 & 32 & 9 \\
\hline${ }^{\mathrm{I}} 04 \ldots$ & 6 & $<1$ & $<.1$ & 92 & 9 & 3,450 & 49 & 17 \\
\hline${ }^{\prime} 07 \ldots$ & 6 & $<1$ & $<.1$ & 47 & 7 & 1,420 & 31 & 8 \\
\hline 07. & 6 & $<1$ & $<.1$ & 40 & 6 & 1,130 & 37 & 7 \\
\hline${ }^{1} 09 \ldots$ & 6 & $<1$ & $<.1$ & 40 & 6 & 1,060 & 24 & 13 \\
\hline $23 \ldots$ & 5 & $<1$ & $<.1$ & 19 & 4 & 389 & 21 & 2 \\
\hline \multicolumn{9}{|l|}{ AUG } \\
\hline 10. & 6 & $<1$ & $<.1$ & 6 & 3 & 53 & $<10$ & $<1$ \\
\hline${ }^{1} 15 \ldots$ & 7 & $<1$ & $<1$ & 12 & 3 & 189 & $<10$ & 1 \\
\hline 19. & 8 & $<.1$ & $<.1$ & 12 & 3 & 150 & $<10$ & 1 \\
\hline$I^{\prime} 22 \ldots$ & 7 & $<.1$ & $<1$ & 9 & 3 & 127 & $<10$ & E.8 \\
\hline${ }^{\mathbf{l}} 25 \ldots$ & 8 & $<.1$ & $<.1$ & 7 & 3 & 84 & $<10$ & E.5 \\
\hline
\end{tabular}


Table 4. Water-quality data for the upper Clark Fork basin, Montana, October 1998 through September 1999 (Continued)

12334550-CLARK FORK AT TURAH BRIDGE, NEAR BONNER, MONT. (Continued)

\begin{tabular}{|c|c|c|c|c|c|c|c|c|}
\hline Date & $\begin{array}{c}\text { Lead, } \\
\text { dissolved } \\
(\mu \mathrm{g} / \mathrm{L})\end{array}$ & $\begin{array}{l}\text { Manga- } \\
\text { nese, } \\
\text { total } \\
\text { recoverable } \\
(\mu \mathrm{g} / \mathrm{L})\end{array}$ & $\begin{array}{l}\text { Manga- } \\
\text { nese, } \\
\text { dissolved } \\
(\mu \mathrm{g} / \mathrm{L})\end{array}$ & $\begin{array}{c}\text { Zinc, } \\
\text { total } \\
\text { recoverable } \\
(\mu \mathrm{g} / \mathrm{L})\end{array}$ & $\begin{array}{c}\text { Zinc, } \\
\text { dissolved } \\
(\mu \mathrm{g} / \mathrm{L})\end{array}$ & $\begin{array}{l}\text { Sediment, } \\
\text { suspended } \\
\text { (mg/L) }\end{array}$ & $\begin{array}{l}\text { Sediment } \\
\text { discharge, } \\
\text { suspended } \\
\text { (ton/d) }\end{array}$ & $\begin{array}{c}\text { Sediment, } \\
\text { suspended } \\
\text { (percent } \\
\text { finer than } \\
0.062 \mathrm{~mm} \text { ) }\end{array}$ \\
\hline \multicolumn{9}{|l|}{ NOV 1998} \\
\hline 18. & $<.5$ & 40 & E3 & 10 & $<20$ & 9 & 26 & 93 \\
\hline \multicolumn{9}{|l|}{ FEB 1999} \\
\hline $24 \ldots$ & $<.5$ & 20 & 7 & 10 & $<20$ & 7 & 15 & 84 \\
\hline \multicolumn{9}{|l|}{ APR } \\
\hline 28 & $<.5$ & 90 & 9 & E38 & $<20$ & 52 & 295 & 66 \\
\hline \multicolumn{9}{|l|}{ MAY } \\
\hline $12 \ldots$ & $<.5$ & 46 & 5 & $<40$ & $<20$ & 18 & 87 & 84 \\
\hline $\mathrm{I}_{25 \ldots}$ & $<.5$ & 194 & 1 & 87 & 4 & 133 & 1,210 & - \\
\hline$I_{26}$ & $<.5$ & 261 & 2 & 113 & 4 & 220 & 2,340 & -. \\
\hline $26 \ldots$ & $<.5$ & 224 & 14 & 264 & $<20$ & 196 & 2,130 & 52 \\
\hline$I_{28} \ldots$ & $<.5$ & 172 & 3 & 73 & 3 & 142 & 1,660 & -- \\
\hline \multicolumn{9}{|l|}{ JUN } \\
\hline${ }^{\prime} 02 \ldots$ & $<.5$ & 157 & 3 & 81 & 5 & 101 & 1,260 & - \\
\hline${ }^{\prime} 04 \ldots$ & $<.5$ & 326 & 2 & 143 & 6 & 320 & 5,540 & - \\
\hline $\mathrm{I}_{07} \ldots$ & $<.5$ & 144 & 3 & 64 & 6 & 105 & 1,520 & -- \\
\hline $07 \ldots$ & $<.5$ & 129 & 9 & 68 & $<20$ & 82 & 1,180 & 62 \\
\hline${ }^{\mathrm{I}} 09 \ldots$ & $<.5$ & 104 & 4 & 60 & 5 & 78 & 960 & -- \\
\hline $23 \ldots$ & $<.5$ & 48 & 6 & $<40$ & $<20$ & 24 & 219 & 65 \\
\hline \multicolumn{9}{|l|}{ AUG } \\
\hline $10 \ldots$ & $<.5$ & 19 & 4 & $<40$ & 2 & 5 & 8.7 & 70 \\
\hline${ }^{1} 15 \ldots$ & $<5$ & 56 & $<1$ & $<31$ & 2 & 12 & 27 & -. \\
\hline${ }^{1} 19 .$. & $<.5$ & 47 & 1 & E19 & 2 & 10 & 20 & - \\
\hline $\mathrm{I}_{22}$. & $<.5$ & 40 & $<1$ & $<31$ & 3 & 10 & 17 & - \\
\hline$I_{25}$ & $<.5$ & 28 & $<1$ & $<31$ & 2 & 6 & 9.7 & - \\
\hline
\end{tabular}


Table 4. Water-quality data for the upper Clark Fork basin, Montana, October 1998 through September 1999 (Continued) 12340000--BLACKFOOT RIVER NEAR BONNER, MONT.

\begin{tabular}{|c|c|c|c|c|c|c|c|c|c|}
\hline Date & Time & $\begin{array}{l}\text { Streamflow, } \\
\text { instan- } \\
\text { tanęous } \\
\left(\mathrm{ft}^{3} / \mathrm{s}\right)\end{array}$ & $\begin{array}{c}\text { Specific } \\
\text { conductance, } \\
\text { onsite } \\
(\mu \mathrm{S} / \mathrm{cm})\end{array}$ & $\begin{array}{c}\text { pH, } \\
\text { onsite } \\
\text { (standard } \\
\text { units) }\end{array}$ & $\begin{array}{l}\text { Temper- } \\
\text { ature, } \\
\text { water } \\
\left({ }^{\circ} \mathrm{C}\right)\end{array}$ & $\begin{array}{c}\text { Hardness, } \\
\text { total } \\
(\mathrm{mg} / \mathrm{L} \text { as } \\
\left.\mathrm{CaCO}_{3}\right)\end{array}$ & $\begin{array}{l}\text { Calcium, } \\
\text { dissolved } \\
(\mathrm{mg} / \mathrm{L})\end{array}$ & $\begin{array}{l}\text { Magne- } \\
\text { sium, } \\
\text { dissolved } \\
(\mathrm{mg} / \mathrm{L})\end{array}$ & $\begin{array}{c}\text { Arsenic, } \\
\text { total } \\
\text { recoverable } \\
(\mu \mathrm{g} / \mathrm{L})\end{array}$ \\
\hline \multicolumn{10}{|l|}{ NOV 1998} \\
\hline 18 & 1600 & 634 & 276 & 8.7 & 4.5 & 130 & 33 & 12 & $<1$ \\
\hline \multicolumn{10}{|l|}{ APR 1999} \\
\hline $29 \ldots$ & 0810 & 3,900 & 163 & 8.3 & 6.0 & 80 & 21 & 7.0 & $<1$ \\
\hline \multicolumn{10}{|l|}{ MAY } \\
\hline $26 \ldots$ & 1435 & 8,990 & 139 & 8.5 & 10.0 & 70 & 18 & 5.7 & 3 \\
\hline \multicolumn{10}{|l|}{ JUN } \\
\hline $09 \ldots$ & 0920 & 5.800 & 171 & 8.2 & 8.5 & 86 & 22 & 7.3 & 1 \\
\hline $23 \ldots$ & 0745 & 5,310 & 160 & 8.2 & 11.0 & 81 & 21 & 6.9 & $<1$ \\
\hline \multicolumn{10}{|l|}{ AUG } \\
\hline $15 \ldots$ & 1010 & 990 & 255 & 8.7 & 13.5 & 130 & 33 & 11 & 1 \\
\hline $15 \ldots$ & 1340 & 990 & 249 & 8.6 & 15.0 & 130 & 32 & 12 & 1 \\
\hline${ }^{\prime} 19 \ldots$ & 1130 & 862 & 259 & 8.5 & 18.0 & 130 & 33 & 12 & 1 \\
\hline$I_{22} \ldots$ & 0915 & 794 & 260 & 8.5 & 16.0 & 130 & 33 & 12 & 1 \\
\hline$I_{25} \ldots$ & 1125 & 750 & 260 & 8.5 & 16.0 & 130 & 33 & 12 & 2 \\
\hline
\end{tabular}

\begin{tabular}{|c|c|c|c|c|c|c|c|c|}
\hline Date & $\begin{array}{c}\text { Arsenic, } \\
\text { dissolved } \\
(\mu \mathrm{g} / \mathrm{L})\end{array}$ & $\begin{array}{l}\text { Cadmium, } \\
\text { total } \\
\text { recoverable } \\
(\mu \mathrm{g} / \mathrm{L})\end{array}$ & $\begin{array}{c}\text { Cadmium, } \\
\text { dissolved } \\
(\mu g / L)\end{array}$ & $\begin{array}{c}\text { Copper, } \\
\text { total } \\
\text { recoverable } \\
(\mu \mathrm{g} / \mathrm{L})\end{array}$ & $\begin{array}{c}\text { Copper, } \\
\text { dissolved } \\
(\mu \mathrm{g} / \mathrm{L})\end{array}$ & $\begin{array}{c}\text { Iron, } \\
\text { total } \\
\text { recoverable } \\
(\mu \mathrm{g} / \mathrm{L})\end{array}$ & $\begin{array}{c}\text { Iron, } \\
\text { dissolved } \\
(\mu \mathrm{g} / \mathrm{L})\end{array}$ & $\begin{array}{c}\text { Lead, } \\
\text { total } \\
\text { recoverable } \\
(\mu \mathrm{g} / \mathrm{L})\end{array}$ \\
\hline \multicolumn{9}{|l|}{ NOV 1998} \\
\hline $18 \ldots$ & $<1$ & $<1$ & .1 & $<1$ & $<1$ & 60 & $<10$ & $<1$ \\
\hline \multicolumn{9}{|l|}{ APR 1999} \\
\hline $29 \ldots$ & $<1$ & $<1$ & $<.1$ & 2 & $<1$ & 338 & 35 & $<1$ \\
\hline \multicolumn{9}{|l|}{ MAY } \\
\hline 26. & $<1$ & $<1$ & $<.1$ & 13 & 2 & 2,410 & 47 & 4 \\
\hline \multicolumn{9}{|l|}{ JUN } \\
\hline 09. & 1 & $<1$ & $<.1$ & 2 & 1 & 416 & 18 & $<1$ \\
\hline 23. & 1 & $<1$ & $<1$ & 3 & 2 & 307 & 14 & $<1$ \\
\hline \multicolumn{9}{|l|}{ AUG } \\
\hline $15 \ldots$ & $<1$ & $<1$ & $<.1$ & $<1$ & $<1$ & 45 & E6 & $<1$ \\
\hline${ }^{t} 15 \ldots$ & $<1$ & $<.1$ & $<.1$ & $<1$ & $<1$ & 47 & $<10$ & I \\
\hline${ }^{1} 19 .$. & 1 & $<1$ & $<.1$ & $<1$ & $<1$ & 32 & $<10$ & $<1$ \\
\hline$t_{22} \ldots$ & $<1$ & $<1$ & $<, 1$ & $<1$ & $<1$ & 34 & $<10$ & $<1$ \\
\hline$t_{25} \ldots$ & $<1$ & $<.1$ & $<1$ & $<1$ & $<1$ & 25 & $<10$ & $<1$ \\
\hline
\end{tabular}

\begin{tabular}{|c|c|c|c|c|c|c|c|c|}
\hline Date & $\begin{array}{c}\text { Lead, } \\
\text { dissolved } \\
(\mu \mathrm{g} / \mathrm{L})\end{array}$ & $\begin{array}{c}\text { Manga- } \\
\text { nese, } \\
\text { total } \\
\text { recoverable } \\
(\mu \mathrm{g} / \mathrm{L})\end{array}$ & $\begin{array}{l}\text { Manga- } \\
\text { nese, } \\
\text { dissolved } \\
(\mu \mathrm{g} / \mathrm{L})\end{array}$ & $\begin{array}{c}\text { Zinc, } \\
\text { total } \\
\text { recoverable } \\
(\mu \mathrm{g} / \mathrm{L})\end{array}$ & $\begin{array}{c}\text { Zinc, } \\
\text { dissolved } \\
(\mu \mathrm{g} / \mathrm{L})\end{array}$ & $\begin{array}{c}\text { Sediment, } \\
\text { suspended } \\
(\mathrm{mg} / \mathrm{L})\end{array}$ & $\begin{array}{c}\text { Sediment } \\
\text { discharge, } \\
\text { suspended } \\
\text { (ton/d) }\end{array}$ & $\begin{array}{c}\text { Sediment, } \\
\text { suspended } \\
\text { (percent } \\
\text { finer than } \\
0.062 \mathrm{~mm} \text { ) }\end{array}$ \\
\hline \multicolumn{9}{|l|}{ NOV 1998} \\
\hline $18 \ldots$ & $<.5$ & $<10$ & E2 & $<10$ & $<20$ & 4 & 6.8 & 82 \\
\hline \multicolumn{9}{|l|}{ APR 1999} \\
\hline $29 \ldots$ & $<.5$ & 31 & E2 & $<40$ & $<20$ & 25 & 263 & 85 \\
\hline \multicolumn{9}{|l|}{ MAY } \\
\hline 26 & $<.5$ & 165 & 5 & $<40$ & $<20$ & 265 & 6,430 & 77 \\
\hline \multicolumn{9}{|l|}{ JUN } \\
\hline 09 & $<.5$ & 30 & 3 & $<40$ & $<20$ & 36 & 564 & 85 \\
\hline 23. & $<.5$ & 20 & E2 & $<40$ & $<20$ & 28 & 401 & 76 \\
\hline \multicolumn{9}{|l|}{ AUG } \\
\hline 15. & $<5$ & 7 & 1 & $<40$ & $<1$ & 4 & 11 & 80 \\
\hline${ }^{t_{15}} \ldots$ & $<5$ & 7 & $<1$ & $<31$ & $<1$ & 2 & 5.3 & - \\
\hline${ }^{1} 19 \ldots$ & $<5$ & 6 & $<1$ & $<31$ & $<1$ & 3 & 7.0 & -- \\
\hline $22 \ldots$ & $<.5$ & 7 & $<1$ & $<31$ & $<1$ & 3 & 6.4 & -- \\
\hline $25 \ldots$ & $<.5$ & 5 & $<1$ & $<31$ & $<1$ & 2 & 4.1 & - \\
\hline
\end{tabular}


Table 4. Water-quality data for the upper Clark Fork basin, Montana, October 1998 through September 1999 (Continued)

12340500--CLARK FORK ABOVE MISSOULA, MONT.

\begin{tabular}{|c|c|c|c|c|c|c|c|c|c|}
\hline Date & Time & $\begin{array}{c}\text { Streamflow, } \\
\text { instan- } \\
\text { taneous } \\
\left(\mathrm{ft}^{3} / \mathrm{s}\right)\end{array}$ & $\begin{array}{c}\text { Specific } \\
\text { conduct- } \\
\text { ance, onsite } \\
(\mu \mathrm{S} / \mathrm{cm})\end{array}$ & $\begin{array}{c}\text { pH, } \\
\text { onsite } \\
\text { (standard } \\
\text { units) }\end{array}$ & $\begin{array}{l}\text { Temper- } \\
\text { ature, } \\
\text { water } \\
\left({ }^{\circ} \mathrm{C}\right)\end{array}$ & $\begin{array}{c}\text { Hardness, } \\
\text { total } \\
(\mathrm{mg} / \mathrm{L} \text { as } \\
\left.\mathrm{CaCO}_{3}\right)\end{array}$ & $\begin{array}{l}\text { Calcium, } \\
\text { dissolved } \\
\text { (mg/L) }\end{array}$ & $\begin{array}{l}\text { Magne- } \\
\text { sium, } \\
\text { dissolved } \\
(\mathrm{mg} / \mathrm{L})\end{array}$ & $\begin{array}{c}\text { Arsenic, } \\
\text { total } \\
\text { recoverable } \\
(\mu \mathrm{g} / \mathrm{L})\end{array}$ \\
\hline \multicolumn{10}{|l|}{ NOV 1998} \\
\hline 19. & 0900 & 1,700 & 330 & 8.4 & 4.0 & 150 & 41 & 12 & 5 \\
\hline \multicolumn{10}{|l|}{ FEB 1999} \\
\hline 24. & 1350 & 1,090 & 326 & 8.6 & 4.0 & 150 & 41 & 12 & 4 \\
\hline \multicolumn{10}{|l|}{ APR } \\
\hline $29 \ldots$ & 0940 & 5,880 & 188 & 8.2 & 6.5 & 88 & 23 & 7.2 & 4 \\
\hline \multicolumn{10}{|l|}{ MAY } \\
\hline $11 \ldots$ & 1730 & 4,710 & 212 & 8.5 & 9.0 & 99 & 26 & 8.1 & 2 \\
\hline${ }^{1} 25 \ldots$ & 1155 & 11,000 & 157 & 8.0 & 13.0 & 75 & 20 & 6.0 & 4 \\
\hline${ }^{3} 26 \ldots$ & 1115 & 12,900 & 146 & 8.1 & 12.0 & 70 & 19 & 5.5 & 5 \\
\hline 26 & 1610 & 13,400 & 142 & 8.1 & 12.0 & 68 & 18 & 5.4 & 5 \\
\hline$' 28 \ldots$ & 1135 & 12,600 & 146 & 8.0 & 12.0 & 70 & 19 & 5.6 & 5 \\
\hline \multicolumn{10}{|l|}{ JUN } \\
\hline${ }^{3} 02 \ldots$ & 1300 & 11,200 & 364 & 8.1 & 11.0 & 76 & 21 & 6.1 & 5 \\
\hline${ }^{1} 04 \ldots$ & 1245 & 14,200 & 156 & 7.7 & 110 & 72 & 20 & 5.6 & 8 \\
\hline${ }^{1} 07 \ldots$ & 1130 & 12,400 & 164 & 7.8 & 10.0 & 76 & 20 & 6.0 & 5 \\
\hline $09 \ldots$ & 1055 & 10,300 & 177 & 8.3 & 8.5 & 83 & 22 & 6.6 & 5 \\
\hline${ }^{1} 09 \ldots$ & 1200 & 10,300 & 177 & 8.1 & 12.0 & 84 & 23 & 6.7 & 4 \\
\hline 23 & 0915 & 8,580 & 173 & 8.2 & 12.0 & 81 & 22 & 6.5 & 3 \\
\hline \multicolumn{10}{|l|}{ AUG } \\
\hline $15 \ldots$ & 1350 & 1,870 & 293 & 8.7 & 14.5 & 140 & 36 & II & 4 \\
\hline $15 \ldots$ & 1515 & 1,830 & 293 & 8.4 & 15.0 & 140 & 37 & 12 & 5 \\
\hline${ }_{1}^{1} 19 \ldots$ & 1245 & 1,610 & 303 & 8.4 & 20.0 & 140 & 38 & 12 & 5 \\
\hline$I_{22} \ldots$ & 1045 & 1,450 & 300 & 8.5 & 18.0 & 140 & 37 & 12 & 5 \\
\hline $\mathrm{l}_{24 \ldots}$ & 1110 & 1,460 & 299 & 8.4 & 17.0 & 140 & 37 & 12 & 5 \\
\hline $1_{25 \ldots}$ & 1240 & 920 & 306 & 8.2 & 18.0 & 140 & 38 & 12 & 7 \\
\hline $\mathrm{I}_{27 \ldots}$ & 3120 & 3,330 & 299 & 8.6 & 19.0 & 140 & 37 & 12 & 5 \\
\hline
\end{tabular}

32 Water-quality, bed-sediment, and biological data (October 1998 through September 1999) and statistical summaries of data for streams in tbe Upper Clark Fork Basin, Montana 
Table 4. Water-quality data for the upper Clark Fork basin, Montana, October 1998 through September 1999 (Continued) 12340500--CLARK FORK ABOVE MISSOULA, MONT. (Continued)

\begin{tabular}{|c|c|c|c|c|c|c|c|c|}
\hline Date & $\begin{array}{c}\text { Arsenic, } \\
\text { dissolved } \\
(\mu \mathrm{g} / \mathrm{L})\end{array}$ & $\begin{array}{l}\text { Cadmium, } \\
\text { total } \\
\text { recoverable } \\
(\mu \mathrm{g} / \mathrm{L})\end{array}$ & $\begin{array}{c}\text { Cadmium, } \\
\text { dissolved } \\
(\mu \mathrm{g} / \mathrm{L})\end{array}$ & $\begin{array}{c}\text { Copper, } \\
\text { total } \\
\text { recoverable } \\
(\mu \mathrm{g} / \mathrm{L})\end{array}$ & $\begin{array}{c}\text { Copper, } \\
\text { dissolved } \\
(\mu \mathrm{g} / \mathrm{L})\end{array}$ & $\begin{array}{c}\text { Iron, } \\
\text { total } \\
\text { recoverable } \\
(\mu \mathrm{g} / \mathrm{L})\end{array}$ & $\begin{array}{c}\text { Iron, } \\
\text { dissolved } \\
(\mu \mathrm{g} / \mathrm{L})\end{array}$ & $\begin{array}{c}\text { Lead, } \\
\text { total } \\
\text { recoverable } \\
(\mu \mathrm{g} / \mathrm{L})\end{array}$ \\
\hline \multicolumn{9}{|l|}{ NOV 1998} \\
\hline $19 \ldots$ & 4 & $<1$ & $<.1$ & 4 & 2 & 100 & $<10$ & $<1$ \\
\hline \multicolumn{9}{|l|}{ FEB 1999} \\
\hline 24 & 3 & $<1$ & $<.1$ & 4 & 2 & 80 & E6 & $<1$ \\
\hline \multicolumn{9}{|l|}{ APR } \\
\hline $29 \ldots$ & 1 & $<1$ & 1 & 8 & 3 & 414 & 46 & 2 \\
\hline \multicolumn{9}{|l|}{ MAY } \\
\hline $11 \ldots$ & 2 & $<1$ & $<.1$ & 6 & 2 & 204 & 15 & $<1$ \\
\hline$I_{25} \ldots$ & 3 & $<1$ & $<.1$ & 19 & 3 & 1,570 & 23 & 4 \\
\hline${ }^{1} 26 \ldots$ & 3 & $<1$ & $<1$ & 20 & 2 & 2,050 & 34 & 5 \\
\hline $26 \ldots$ & 2 & $<1$ & $<1$ & 22 & 2 & 2,200 & 49 & 5 \\
\hline${ }^{I} 28$ & 3 & $<1$ & $<1$ & 20 & 3 & 1,450 & 32 & 4 \\
\hline \multicolumn{9}{|l|}{ rUN } \\
\hline${ }^{\prime} 02 \ldots$ & 3 & $<1$ & $<.1$ & 32 & 3 & 912 & 23 & 4 \\
\hline${ }^{1} 04 \ldots$ & 3 & $<1$ & $<.1$ & 50 & 5 & 1,860 & 29 & 8 \\
\hline${ }^{1} 07 \ldots$ & 3 & $<1$ & $<1$ & 25 & 4 & 970 & 23 & 4 \\
\hline $09 \ldots$ & 3 & $<1$ & $<.1$ & 18 & 4 & 607 & 24 & 3 \\
\hline${ }^{1} 09 \ldots$ & 3 & $<1$ & $<.1$ & 32 & 4 & 709 & 19 & 4 \\
\hline $23 \ldots$ & 2 & $<1$ & $<.1$ & 11 & 3 & 392 & 50 & 2 \\
\hline \multicolumn{9}{|l|}{ AUG } \\
\hline $15 \ldots$ & 3 & $<1$ & $<.1$ & 7 & 3 & 110 & E7 & $<1$ \\
\hline${ }^{1} 15 \ldots$ & 4 & $<.1$ & $<1$ & 5 & 2 & 107 & $<10$ & $<1$ \\
\hline${ }^{1} 19 \ldots$ & 4 & $<1$ & $<1$ & 6 & 2 & 138 & $<10$ & E.8 \\
\hline $1_{22 \ldots}$ & 5 & $<.1$ & $<.1$ & 6 & 2 & 163 & $<10$ & E.7 \\
\hline${ }^{1} 24 \ldots$ & 4 & $<.1$ & $<.1$ & 8 & 1 & 210 & E6 & 1 \\
\hline${ }^{1} 25 \ldots$ & 5 & .4 & $<1$ & 25 & 2 & 601 & $<10$ & 9 \\
\hline${ }^{\mathrm{I}} 27 \ldots$ & 4 & $<.1$ & $<1$ & 3 & 1 & 71 & $<10$ & $<1$ \\
\hline
\end{tabular}


Table 4. Water-quality data for the upper Clark Fork basin, Montana, October 1998 through September 1999 (Continued) 12340500--CLARK FORK ABOVE MISSOULA, MONT. (Continued)

\begin{tabular}{|c|c|c|c|c|c|c|c|c|}
\hline Date & $\begin{array}{l}\text { Lead, } \\
\text { dissolved } \\
(\mu \mathrm{g} / \mathrm{L})\end{array}$ & $\begin{array}{c}\text { Manga- } \\
\text { nese, } \\
\text { total } \\
\text { recoverable } \\
(\mu \mathrm{g} / \mathrm{L})\end{array}$ & $\begin{array}{l}\text { Manga- } \\
\text { nese, } \\
\text { dissolved } \\
(\mu \mathrm{g} / \mathrm{L})\end{array}$ & $\begin{array}{c}\text { Zinc, } \\
\text { total } \\
\text { recoverable } \\
(\mu \mathrm{g} / \mathrm{L})\end{array}$ & $\begin{array}{l}\text { Zinc, } \\
\text { dissolved } \\
(\mu \mathrm{g} / \mathrm{L})\end{array}$ & $\begin{array}{c}\text { Sediment, } \\
\text { suspended } \\
\text { (mg/L) }\end{array}$ & $\begin{array}{l}\text { Sediment } \\
\text { discharge, } \\
\text { suspended } \\
\text { (ton/d) }\end{array}$ & $\begin{array}{c}\text { Sediment, } \\
\text { suspended } \\
\text { (percent } \\
\text { finer than } \\
0.062 \mathrm{~mm} \text { ) }\end{array}$ \\
\hline \multicolumn{9}{|l|}{ NOV 1998} \\
\hline $19 \ldots$ & $<.5$ & 30 & 13 & $<10$ & $<20$ & 5 & 23 & 95 \\
\hline \multicolumn{9}{|l|}{ FEB 1999} \\
\hline 24 & $<.5$ & 40 & 36 & $<10$ & E7 & 3 & 8.8 & 95 \\
\hline \multicolumn{9}{|l|}{ APR } \\
\hline $29 .$. & $<.5$ & 47 & 12 & $<40$ & $<20$ & 25 & 397 & 92 \\
\hline \multicolumn{9}{|l|}{ MAY } \\
\hline $11 .$. & $<.5$ & 26 & 13 & $<40$ & $<20$ & 13 & 165 & 92 \\
\hline $\mathrm{I}_{25}$. & $<.5$ & 126 & 1 & E28 & 1 & 110 & 3,270 & -- \\
\hline 26. & $<.5$ & 153 & 2 & E33 & 1 & 154 & 5,360 & - \\
\hline $26 \ldots$ & $<.5$ & 176 & 15 & 40 & $<20$ & 200 & 7,240 & 85 \\
\hline${ }^{1} 28 \ldots$ & $<.5$ & 110 & 3 & E30 & 1 & 109 & 3,710 & -- \\
\hline \multicolumn{9}{|l|}{ JUN } \\
\hline $\mathrm{I}_{02} \ldots$ & $<.5$ & 84 & 4 & 42 & 2 & 60 & 1,810 & - \\
\hline${ }^{I} 04 \ldots$ & $<.5$ & 169 & I & 82 & 4 & 128 & 4,910 & - \\
\hline${ }^{1} 07 \ldots$ & $<.5$ & 82 & 3 & E37 & 3 & 68 & 2,280 & - \\
\hline $09 \ldots$ & $<.5$ & 66 & 9 & $\mathrm{E} 22$ & $<20$ & 43 & 1,200 & 83 \\
\hline${ }^{1} 09 \ldots$ & $<.5$ & 62 & 4 & E34 & 3 & 42 & 1,170 & - \\
\hline $23 \ldots$ & $<.5$ & 39 & 11 & $<40$ & $<20$ & 28 & 649 & 89 \\
\hline \multicolumn{9}{|l|}{ AUG } \\
\hline $15 \ldots$ & $<.5$ & 24 & 11 & $<40$ & I & 9 & 45 & 95 \\
\hline $15 \ldots$ & $<.5$ & 23 & $<1$ & $<31$ & 1 & 6 & 30 & -. \\
\hline${ }^{1} 19 \ldots$ & $<.5$ & 46 & $<1$ & $<31$ & $<1$ & 8 & 35 & - \\
\hline $1_{22 \ldots}$ & $<.5$ & 49 & 1 & $<31$ & 2 & 9 & 35 & - \\
\hline${ }^{\mathrm{l}} 24 \ldots$ & $<.5$ & 57 & 6 & $<31$ & 1 & 13 & 51 & -- \\
\hline $\mathrm{I}_{25} \ldots$ & $<.5$ & 156 & 1 & 32 & 2 & 32 & 79 & - \\
\hline $\mathrm{I}_{27 \ldots}$ & $<.5$ & 26 & $<1$ & $<31$ & $<1$ & 8 & 29 & - \\
\hline
\end{tabular}

'Supplemental samples collected to better define changes in trace-element concentrations and transport during extended high flows, and during the lowering of Milltown Reservoir water levels for dam maintenance. 
Table 5. Daily streamflow and suspended-sediment data for Clark Fork at Deer Lodge. Montana. October 1998 through September 1999 [Abbreviations: $\mathrm{ft}^{3} / \mathrm{s}$, cubic feet per second; e, estimated; $\mathrm{mg} / \mathrm{L}$, milligrams per liter; ton/d, tons per day. Symbol: ---, no data]

\begin{tabular}{|c|c|c|c|c|c|c|c|c|c|}
\hline \multirow[b]{2}{*}{ Day } & \multirow{2}{*}{$\begin{array}{c}\text { Mean } \\
\text { stream- } \\
\text { flow } \\
\left(\mathrm{fr}^{3} / \mathrm{s}\right)\end{array}$} & \multicolumn{2}{|c|}{ Suspended sediment } & \multirow{2}{*}{$\begin{array}{c}\text { Mean } \\
\text { stream- } \\
\text { flow } \\
\left(\mathbf{f t}^{3} / \mathbf{s}\right)\end{array}$} & \multicolumn{2}{|c|}{ Suspended sediment } & \multirow[b]{2}{*}{$\begin{array}{c}\text { Mean } \\
\text { stream- } \\
\text { flow } \\
\left(\mathrm{ft}^{3} / \mathrm{s}\right)\end{array}$} & \multicolumn{2}{|c|}{ Suspended sediment } \\
\hline & & $\begin{array}{c}\text { Mean } \\
\text { concen- } \\
\text { tration } \\
(\mathrm{mg} / \mathrm{L})\end{array}$ & $\begin{array}{l}\text { Dis- } \\
\text { charge } \\
\text { (ton/d) }\end{array}$ & & $\begin{array}{l}\text { Mean } \\
\text { concen- } \\
\text { tration } \\
(\mathrm{mg} / \mathrm{L})\end{array}$ & $\begin{array}{l}\text { Dis- } \\
\text { charge } \\
\text { (ton/d) }\end{array}$ & & $\begin{array}{l}\text { Mean } \\
\text { concen- } \\
\text { tration } \\
(\mathrm{mg} / \mathrm{L})\end{array}$ & $\begin{array}{c}\text { Dis- } \\
\text { charge } \\
\text { (ton/d) }\end{array}$ \\
\hline \multicolumn{10}{|c|}{1998} \\
\hline & \multicolumn{3}{|c|}{ October } & \multicolumn{3}{|c|}{ November } & \multicolumn{3}{|c|}{ December } \\
\hline I & 225 & 20 & 12 & 248 & 11 & 7.4 & 248 & 16 & 11 \\
\hline 2 & 240 & 25 & 16 & 248 & 11 & 7.4 & 248 & 17 & 11 \\
\hline 3 & 252 & 29 & 20 & 253 & 11 & 7.5 & 252 & 19 & 13 \\
\hline 4 & 258 & 29 & 20 & 255 & 11 & 7.6 & 250 & 21 & 14 \\
\hline 5 & 257 & 27 & 19 & 258 & 13 & 9.1 & 230 & 21 & 13 \\
\hline 6 & 272 & 36 & 26 & 273 & 16 & 12 & 221 & 21 & 13 \\
\hline 7 & 269 & 21 & 15 & 270 & 17 & 12 & 222 & 20 & 12 \\
\hline 8 & 264 & 21 & 15 & 277 & 18 & 13 & 217 & 20 & 12 \\
\hline 9 & 261 & 21 & 15 & 291 & 18 & 14 & 225 & 20 & 12 \\
\hline 10 & 272 & 22 & 16 & 304 & 19 & 16 & 222 & 20 & 12 \\
\hline 11 & 289 & 22 & 17 & 290 & 20 & 16 & 232 & 20 & 13 \\
\hline 12 & 272 & 22 & 16 & 297 & 20 & 16 & 244 & 20 & 13 \\
\hline 13 & 281 & 22 & 17 & 300 & 23 & 19 & 254 & 20 & 14 \\
\hline 14 & 282 & 21 & 16 & 321 & 25 & 22 & 262 & 20 & 14 \\
\hline 15 & 288 & 20 & 16 & 313 & 25 & 21 & 246 & 20 & 13 \\
\hline 16 & 283 & 17 & 13 & 293 & 20 & 16 & 246 & 20 & 13 \\
\hline 17 & 274 & 15 & 11 & 286 & 16 & 12 & 252 & 20 & 14 \\
\hline 18 & 272 & 14 & 10 & 300 & 16 & 13 & 230 & 19 & 12 \\
\hline 19 & 269 & 14 & 10 & 300 & 16 & 13 & 163 & 19 & 8.4 \\
\hline 20 & 265 & 15 & 11 & 291 & 16 & 13 & 149 & 20 & 8.0 \\
\hline 21 & 265 & 18 & 13 & 295 & 16 & 13 & el 40 & 22 & 8.3 \\
\hline 22 & 265 & 19 & 14 & 315 & 16 & 14 & el 40 & 22 & 8.3 \\
\hline 23 & 260 & 18 & 13 & 302 & 17 & 14 & el50 & 21 & 8.5 \\
\hline 24 & 252 & 16 & 11 & 300 & 16 & 13 & el 150 & 19 & 7.7 \\
\hline 25 & 255 & 13 & 9.0 & 290 & 16 & 13 & el60 & 17 & 7.3 \\
\hline 26 & 258 & 11 & 7.7 & 276 & 15 & 11 & 175 & 16 & 7.6 \\
\hline 27 & 257 & 11 & 7.6 & 254 & 15 & 10 & 198 & 17 & 91 \\
\hline 28 & 257 & 11 & 7.6 & 251 & 15 & 10 & 215 & 23 & 13 \\
\hline 29 & 254 & 14 & 9.6 & 257 & 16 & 11 & 241 & 30 & 20 \\
\hline 30 & 251 & 11 & 7.5 & 253 & 16 & 11 & 276 & 30 & 22 \\
\hline 31 & 249 & 11 & 7.4 & -. & - & -- & 259 & 30 & 21 \\
\hline TOTAL & 8,168 & - & 418.4 & 8,461 & - & 3870 & 6,717 & -- & 378.2 \\
\hline MEAN & 263 & 19 & 13 & 282 & 17 & 13 & 217 & 21 & 12 \\
\hline MAX & 289 & 36 & 26 & 321 & 25 & 22 & 276 & 30 & 22 \\
\hline MIN & 225 & 11 & 7.4 & 248 & 11 & 74 & 140 & 16 & 7.3 \\
\hline
\end{tabular}


Table 5. Daily streamflow and suspended-sediment data for C̀lark Fork at Deer Lodge, Montana, October 1998 through September 1999 (Continued)

\begin{tabular}{|c|c|c|c|c|c|c|c|c|c|}
\hline \multirow[b]{2}{*}{ Day } & \multirow[b]{2}{*}{$\begin{array}{c}\text { Mean } \\
\text { stream- } \\
\text { flow } \\
\left(\mathrm{ft}^{3} / \mathrm{s}\right)\end{array}$} & \multicolumn{2}{|c|}{ Suspended sediment } & \multirow[b]{2}{*}{$\begin{array}{c}\text { Mean } \\
\text { stream- } \\
\text { flow } \\
\left(\mathrm{ft}^{3} / \mathrm{s}\right)\end{array}$} & \multicolumn{2}{|c|}{ Suspended sediment } & \multirow[b]{2}{*}{$\begin{array}{c}\text { Mean } \\
\text { stream- } \\
\text { flow } \\
\left(\mathbf{f i}^{3} / \mathbf{s}\right)\end{array}$} & \multicolumn{2}{|c|}{ Suspended sediment } \\
\hline & & $\begin{array}{l}\text { Mean } \\
\text { concen- } \\
\text { tration } \\
(\mathrm{mg} / \mathrm{L})\end{array}$ & $\begin{array}{l}\text { Dis- } \\
\text { charge } \\
\text { (ton/d) }\end{array}$ & & $\begin{array}{c}\text { Mean } \\
\text { concen- } \\
\text { tration } \\
(\mathrm{mg} / \mathrm{L})\end{array}$ & $\begin{array}{l}\text { Dis- } \\
\text { charge } \\
\text { (ton/d) }\end{array}$ & & $\begin{array}{l}\text { Mean } \\
\text { concen- } \\
\text { tration } \\
(\mathrm{mg} / \mathrm{L})\end{array}$ & $\begin{array}{l}\text { Dis- } \\
\text { charge } \\
\text { (ton/d) }\end{array}$ \\
\hline \multicolumn{10}{|c|}{1999} \\
\hline & \multicolumn{3}{|c|}{ January } & \multicolumn{3}{|c|}{ February } & \multicolumn{3}{|c|}{ March } \\
\hline 1 & 260 & 30 & 21 & e220 & 20 & 12 & 280 & 62 & 47 \\
\hline 2 & 259 & 30 & 21 & 220 & 20 & 12 & 241 & 30 & 20 \\
\hline 3 & e230 & 31 & 19 & 248 & 28 & 19 & 227 & 18 & 11 \\
\hline 4 & 242 & 33 & 22 & 227 & 23 & 14 & 206 & 20 & 11 \\
\hline 5 & 253 & 37 & 25 & 234 & 21 & 13 & 206 & 21 & 12 \\
\hline 6 & 260 & 39 & 27 & 225 & 23 & 14 & 216 & 24 & 14 \\
\hline 7 & 256 & 38 & 26 & 231 & 28 & 17 & 213 & 22 & 13 \\
\hline 8 & 248 & 35 & 23 & 230 & 31 & 19 & 210 & 26 & 15 \\
\hline 9 & 247 & 30 & 20 & 242 & 29 & 19 & 217 & 23 & 13 \\
\hline I0 & 251 & 25 & 17 & 248 & 26 & 17 & 221 & 24 & 14 \\
\hline 11 & 266 & 21 & 15 & 236 & 25 & 16 & 224 & 29 & 18 \\
\hline 12 & 264 & 20 & 14 & 246 & 25 & 17 & 221 & 21 & 13 \\
\hline 13 & 257 & 19 & 13 & 262 & 25 & 18 & 226 & 24 & 15 \\
\hline 14 & 255 & 19 & 13 & 237 & 25 & 16 & 239 & 26 & 17 \\
\hline 15 & 266 & 19 & 14 & $23 \mathrm{I}$ & 25 & 16 & 247 & 33 & 22 \\
\hline 16 & 257 & 20 & 14 & 232 & 25 & 16 & 250 & 34 & 23 \\
\hline 17 & 248 & 20 & 13 & 233 & 24 & 15 & 236 & 24 & 15 \\
\hline 18 & 249 & 20 & 13 & 230 & 22 & 14 & 234 & 31 & 20 \\
\hline 19 & 252 & 20 & 14 & 232 & 20 & 13 & 244 & 32 & 21 \\
\hline 20 & 252 & 19 & 13 & 226 & 18 & 11 & 248 & 40 & 27 \\
\hline 21 & 251 & 18 & 12 & 228 & 16 & 98 & 250 & 40 & 27 \\
\hline 22 & 245 & 17 & 11 & 228 & 17 & 10 & 271 & 54 & 40 \\
\hline 23 & 241 & 16 & 10 & 234 & 19 & 12 & 286 & 55 & 42 \\
\hline 24 & 236 & 17 & 11 & 243 & 24 & 16 & 290 & 52 & 41 \\
\hline 25 & 230 & 24 & 15 & 267 & 24 & 17 & 305 & 69 & 57 \\
\hline 26 & 237 & 36 & 23 & 268 & 22 & 16 & 305 & 59 & 49 \\
\hline 27 & 238 & 35 & 22 & 246 & 23 & 15 & 298 & 42 & 34 \\
\hline 28 & 250 & 30 & 20 & 265 & 28 & 20 & 280 & 32 & 24 \\
\hline 29 & 242 & 26 & 17 & - & - & - & 281 & 34 & 26 \\
\hline 30 & 251 & 23 & 16 & - & - & - & 274 & 36 & 27 \\
\hline 31 & 234 & 21 & 13 & -- & $\cdots$ & -- & 271 & 34 & 25 \\
\hline TOTAL & 7,727 & - & 527 & 6,669 & $\cdots$ & 423.8 & 7,717 & -. & 753 \\
\hline MEAN & 249 & 25 & 17 & 238 & 23 & 15 & 249 & 35 & 24 \\
\hline MAX & 266 & 39 & 27 & 268 & 31 & 20 & 305 & 69 & 57 \\
\hline MIN & 230 & 16 & 10 & 220 & 16 & 9.8 & 206 & 18 & 11 \\
\hline
\end{tabular}


Table 5. Daily streamflow and suspended-sediment data for Clark Fork at Deer Lodge, Montana, October 1998 through September 1999 (Continued)

\begin{tabular}{|c|c|c|c|c|c|c|c|c|c|}
\hline \multirow[b]{2}{*}{ Day } & \multirow[b]{2}{*}{$\begin{array}{c}\text { Mean } \\
\text { stream- } \\
\text { flow } \\
\left(\mathrm{ft}^{3} / \mathrm{s}\right)\end{array}$} & \multicolumn{2}{|c|}{ Suspended sediment } & \multirow[b]{2}{*}{$\begin{array}{c}\text { Mean } \\
\text { stream- } \\
\text { flow } \\
\left(\mathrm{ft}^{3} / \mathrm{s}\right)\end{array}$} & \multicolumn{2}{|c|}{ Suspended sediment } & \multirow[b]{2}{*}{$\begin{array}{l}\text { Mean } \\
\text { stream- } \\
\text { flow } \\
\left(\mathrm{ft}^{3} / \mathrm{s}\right)\end{array}$} & \multicolumn{2}{|c|}{ Suspended sediment } \\
\hline & & $\begin{array}{l}\text { Mean } \\
\text { concen- } \\
\text { tration } \\
(\mathrm{mg} / \mathrm{L})\end{array}$ & $\begin{array}{c}\text { Dis- } \\
\text { charge } \\
\text { (ton/d) }\end{array}$ & & $\begin{array}{l}\text { Mean } \\
\text { concen- } \\
\text { tration } \\
(\mathrm{mg} / \mathrm{L})\end{array}$ & $\begin{array}{c}\text { Dis- } \\
\text { charge } \\
\text { (ton/d) }\end{array}$ & & $\begin{array}{l}\text { Mean } \\
\text { concen- } \\
\text { tration } \\
(\mathrm{mg} / \mathrm{L})\end{array}$ & $\begin{array}{l}\text { Dis- } \\
\text { charge } \\
\text { (ton/d) }\end{array}$ \\
\hline \multicolumn{10}{|c|}{1999} \\
\hline & \multicolumn{3}{|c|}{ April } & \multicolumn{3}{|c|}{ May } & \multicolumn{3}{|c|}{ June } \\
\hline 1 & 249 & 25 & 17 & 273 & 22 & 16 & 606 & 85 & 139 \\
\hline 2 & 238 & 27 & 17 & 280 & 18 & 14 & 620 & 90 & 151 \\
\hline 3 & 246 & 27 & 18 & 292 & 22 & 17 & 681 & 102 & 188 \\
\hline 4 & 243 & 24 & 16 & 303 & 39 & 32 & 796 & 112 & $24 I$ \\
\hline 5 & 238 & 23 & 15 & 289 & 23 & 18 & 773 & 90 & 188 \\
\hline 6 & 232 & 24 & 15 & 274 & 20 & 15 & 703 & 80 & 152 \\
\hline 7 & 226 & 22 & 13 & 266 & 22 & 16 & 655 & 75 & 133 \\
\hline 8 & 226 & 21 & 13 & 258 & 18 & 13 & 614 & 61 & 101 \\
\hline 9 & 223 & 22 & 13 & 274 & 18 & 13 & 585 & 50 & 79 \\
\hline 10 & 219 & 19 & 11 & 287 & 18 & 14 & 491 & 46 & 61 \\
\hline 11 & 218 & 20 & 12 & 280 & 18 & 14 & 429 & 37 & 43 \\
\hline 12 & 217 & 25 & 15 & 265 & 16 & 11 & 390 & 34 & 36 \\
\hline 13 & 214 & 19 & 11 & 258 & 16 & 11 & 376 & 34 & 35 \\
\hline 14 & 210 & 18 & 10 & 245 & 14 & 9.3 & 390 & 39 & 41 \\
\hline 15 & 212 & 20 & 11 & 241 & 16 & 10 & 444 & 61 & 73 \\
\hline 16 & 212 & 20 & 11 & 245 & 17 & 11 & 525 & 79 & 112 \\
\hline 17 & 214 & 22 & 13 & 238 & 16 & 10 & 641 & 97 & 168 \\
\hline 18 & 215 & 17 & 9.9 & 230 & 16 & 9.9 & 697 & 95 & 179 \\
\hline 19 & 219 & 16 & 9.5 & 253 & 24 & 16 & 738 & 94 & 187 \\
\hline 20 & 220 & 18 & 11 & 262 & 27 & 19 & 725 & 80 & 157 \\
\hline 21 & 245 & 23 & 15 & 278 & 31 & 23 & 649 & 58 & 102 \\
\hline 22 & 246 & 21 & 14 & 324 & 70 & 61 & 660 & 51 & 91 \\
\hline 23 & 244 & 15 & 9.9 & 363 & 90 & 88 & 618 & 46 & 77 \\
\hline 24 & 240 & 16 & 10 & 333 & 70 & 63 & 540 & 39 & 57 \\
\hline 25 & 237 & 12 & 7.7 & 352 & 92 & 87 & 508 & 36 & 49 \\
\hline 26 & 237 & 11 & 7.0 & 408 & 135 & 149 & 492 & 34 & 45 \\
\hline 27 & 249 & 13 & 8.7 & 483 & 205 & 267 & 437 & 26 & 31 \\
\hline 28 & 278 & 29 & 22 & 488 & 150 & 198 & 396 & 18 & 19 \\
\hline 29 & 281 & 20 & 15 & 547 & 160 & 236 & 360 & 12 & 12 \\
\hline 30 & 276 & 22 & 16 & 733 & 230 & 455 & 346 & 11 & 10 \\
\hline 31 & - & -- & $\cdots$ & 690 & 135 & 252 & - & $\cdots$ & $\cdots$ \\
\hline TOTAL & 7,024 & $\cdots$ & 3867 & 10,312 & -- & 2,1682 & 16,885 & $\ldots$ & 2,957 \\
\hline MEAN & 234 & 20 & 13 & 333 & 57 & 70 & 563 & 59 & 99 \\
\hline MAX & 281 & 29 & 22 & 733 & 230 & 455 & 796 & 112 & 241 \\
\hline MIN & 210 & 11 & 7.0 & 230 & 14 & 9.3 & 346 & 11 & 10 \\
\hline
\end{tabular}


Table 5. Daily streamflow and suspended-sediment data for Clark Fork at Deer Lodge, Montana, October 1998 through September 1999 (Continued)

\begin{tabular}{|c|c|c|c|c|c|c|c|c|c|}
\hline \multirow[b]{2}{*}{ Day } & \multirow{2}{*}{$\begin{array}{c}\text { Mean } \\
\text { stream- } \\
\text { flow } \\
\left(\mathrm{ft}^{3} / \mathrm{s}\right)\end{array}$} & \multicolumn{2}{|c|}{ Suspended sediment } & \multirow{2}{*}{$\begin{array}{c}\text { Mean } \\
\text { stream- } \\
\text { flow } \\
\left(\mathrm{ft}^{3} / \mathrm{s}\right)\end{array}$} & \multicolumn{2}{|c|}{ Suspended sediment } & \multirow{2}{*}{$\begin{array}{c}\text { Mean } \\
\text { stream- } \\
\text { flow } \\
\left(\mathrm{ft}^{3} / \mathrm{s}\right)\end{array}$} & \multicolumn{2}{|c|}{ Suspended sediment } \\
\hline & & $\begin{array}{l}\text { Mean } \\
\text { concen- } \\
\text { tration } \\
(\mathrm{mg} / \mathrm{L})\end{array}$ & $\begin{array}{c}\text { Dis- } \\
\text { charge } \\
\text { (ton/d) }\end{array}$ & & $\begin{array}{l}\text { Mean } \\
\text { concen- } \\
\text { tration } \\
\text { (mg/L) }\end{array}$ & $\begin{array}{c}\text { Dis- } \\
\text { charge } \\
\text { (ton/d) }\end{array}$ & & $\begin{array}{l}\text { Mean } \\
\text { concen- } \\
\text { tration } \\
\text { (mg/L) }\end{array}$ & $\begin{array}{c}\text { Dis- } \\
\text { charge } \\
\text { (ton/d) }\end{array}$ \\
\hline \multicolumn{10}{|c|}{1999} \\
\hline & \multicolumn{3}{|c|}{ July } & \multicolumn{3}{|c|}{ August } & \multicolumn{3}{|c|}{ September } \\
\hline 1 & 351 & 11 & 10 & 52 & 7 & .98 & 118 & 8 & 2.5 \\
\hline 2 & 327 & 10 & 8.8 & 57 & 16 & 2.5 & 122 & 8 & 2.6 \\
\hline 3 & 307 & 9 & 7.5 & 62 & 18 & 3.0 & 135 & 9 & 3.3 \\
\hline 4 & 288 & 7 & 5.4 & 68 & 21 & 3.9 & 153 & 9 & 3.7 \\
\hline 5 & 274 & 5 & 3.7 & 97 & 49 & 13 & 166 & 9 & 4.0 \\
\hline 6 & 247 & 5 & 3.3 & 81 & 9 & 2.0 & 161 & 8 & 3.5 \\
\hline 7 & 228 & 6 & 3.7 & 82 & 8 & 1.8 & 157 & 8 & 3.4 \\
\hline 8 & 217 & 6 & 3.5 & 78 & 7 & 1.5 & 160 & 8 & 3.5 \\
\hline 9 & 207 & 5 & 2.8 & 74 & 6 & 1.2 & 165 & 7 & 3.1 \\
\hline 10 & 192 & 5 & 2.6 & 75 & 5 & 1.0 & 165 & 7 & 3.1 \\
\hline 11 & 181 & 5 & 2.4 & 80 & 7 & 1.5 & 150 & 8 & 3.2 \\
\hline 12 & 173 & 6 & 2.8 & 113 & 19 & 5.8 & 153 & 10 & 4.1 \\
\hline 13 & 156 & 6 & 2.5 & 126 & 23 & 7.8 & 160 & 14 & 6.0 \\
\hline 14 & 143 & 6 & 2.3 & 116 & 14 & 4.4 & 157 & 15 & 6.4 \\
\hline 15 & 124 & 5 & 1.7 & 111 & 13 & 3.9 & 157 & 13 & 5,5 \\
\hline 16 & 121 & 4 & I. 3 & 110 & 13 & 3.9 & 150 & 11 & 4.5 \\
\hline 17 & 121 & 3 & .98 & 107 & 13 & 3.8 & 150 & 10 & 4.1 \\
\hline 18 & 121 & 4 & 1.3 & 101 & 12 & 3.3 & 156 & 10 & 4.2 \\
\hline 19 & 110 & 4 & 1.2 & 96 & 12 & 3.1 & 163 & 10 & 4.4 \\
\hline 20 & 103 & 5 & 1.4 & 91 & 11 & 2.7 & 162 & 11 & 4.8 \\
\hline 21 & 104 & 6 & 1.7 & 91 & 10 & 2.5 & 154 & 14 & 58 \\
\hline 22 & 95 & 5 & 1.3 & 99 & 10 & 2.7 & 151 & 18 & 7.3 \\
\hline 23 & 88 & 4 & .95 & 96 & 9 & 2.3 & 144 & 14 & 5.4 \\
\hline 24 & 78 & 4 & 84 & 97 & 9 & 2.4 & 132 & 10 & 36 \\
\hline 25 & 78 & 3 & 63 & 102 & 7 & 1.9 & 145 & 9 & 3.5 \\
\hline 26 & 74 & 3 & .60 & 101 & 7 & 1.9 & 150 & 10 & 4.1 \\
\hline 27 & 67 & 3 & .54 & 95 & 7 & 1.8 & 154 & 11 & 4.6 \\
\hline 28 & 63 & 4 & .68 & 100 & 9 & 2.4 & 160 & 11 & 4.8 \\
\hline 29 & 63 & 4 & 68 & 117 & 10 & 3.2 & 166 & 12 & 5.4 \\
\hline 30 & 63 & 3 & .51 & 107 & 8 & 2.3 & 163 & 12 & 5.3 \\
\hline 31 & 59 & 4 & .64 & 112 & 8 & 2.4 & -- & -- & -- \\
\hline TOTAL & 4,823 & $\cdots$ & 78.25 & 2,894 & --- & 96.88 & 4,579 & --- & 129.7 \\
\hline MEAN & 156 & 5 & 2.5 & 934 & 12 & 3.1 & 153 & 10 & 4.3 \\
\hline MAX & 351 & 11 & 10 & 126 & 49 & 13 & 166 & 18 & 7.3 \\
\hline MIN & 59 & 3 & .51 & 52 & 5 & .98 & 118 & 7 & 2.5 \\
\hline
\end{tabular}

TOTAL FOR WATER YEAR 1999:

STREAMFLOW---91,976 $\mathrm{ft}^{3} / \mathrm{s}$

SEDIMENT DISCHARGE---8,704.13 tons

38 Water-quality, bed-sediment, and biological data (October 1998 through September 1999) and statistical summaries of data for streams in the Upper Clark Fork Basin, Montana 
Table 6. Daily streamflow and suspended-sediment data for Clark Fork at Turah Bridge, near Bonner, Montana, October 1998 through September 1999

[Abbreviations: $\mathrm{ft}^{3} / \mathrm{s}$, cubic feet per second; $\mathrm{mg} / \mathrm{L}$, milligrams per liter; ton/d, tons per day. Symbol: --- no data]

\begin{tabular}{|c|c|c|c|c|c|c|c|c|c|}
\hline \multirow[b]{2}{*}{ Day } & \multirow{2}{*}{$\begin{array}{c}\text { Mean } \\
\text { stream- } \\
\text { flow } \\
\left(\mathrm{ft}^{3} / \mathrm{s}\right)\end{array}$} & \multicolumn{2}{|c|}{ Suspended sediment } & \multirow{2}{*}{$\begin{array}{c}\text { Mean } \\
\text { stream- } \\
\text { flow } \\
\left(\mathrm{ft}^{3} / \mathrm{s}\right)\end{array}$} & \multicolumn{2}{|c|}{ Suspended sediment } & \multirow[b]{2}{*}{$\begin{array}{c}\text { Mean } \\
\text { stream- } \\
\text { flow } \\
\left(\mathrm{ft}^{3} / \mathrm{s}\right)\end{array}$} & \multicolumn{2}{|c|}{ Suspended sediment } \\
\hline & & $\begin{array}{l}\text { Mean } \\
\text { concen- } \\
\text { tration } \\
(\mathrm{mg} / \mathrm{L})\end{array}$ & $\begin{array}{c}\text { Dis- } \\
\text { charge } \\
\text { (ton/d) }\end{array}$ & & $\begin{array}{l}\text { Mean } \\
\text { concen- } \\
\text { tration } \\
(\mathrm{mg} / \mathrm{L})\end{array}$ & $\begin{array}{l}\text { Dis- } \\
\text { charge } \\
\text { (ton/d) }\end{array}$ & & $\begin{array}{l}\text { Mean } \\
\text { concen- } \\
\text { tration } \\
(\mathrm{mg} / \mathrm{L})\end{array}$ & $\begin{array}{c}\text { Dis- } \\
\text { charge } \\
\text { (ton/d) }\end{array}$ \\
\hline \multicolumn{10}{|c|}{1998} \\
\hline & \multicolumn{3}{|c|}{ October } & \multicolumn{3}{|c|}{ November } & \multicolumn{3}{|c|}{ December } \\
\hline 1 & 955 & 12 & 31 & 990 & 5 & 13 & 1,080 & 7 & 20 \\
\hline 2 & 982 & 11 & 29 & 996 & 5 & 13 & 1,090 & 8 & 24 \\
\hline 3 & 1,030 & 11 & 31 & 998 & 5 & 13 & 1,130 & 8 & 24 \\
\hline 4 & 1,050 & 11 & 31 & 998 & 4 & 11 & 1,110 & 7 & 21 \\
\hline 5 & 1,060 & 11 & 31 & 989 & 4 & 11 & 1,050 & 5 & 14 \\
\hline 6 & 1,050 & 11 & 31 & 996 & 3 & 8.1 & 942 & 4 & 10 \\
\hline 7 & 1,060 & 12 & 34 & 1,000 & 3 & 8.1 & 896 & 5 & 12 \\
\hline 8 & 1,050 & 13 & 37 & 1,010 & 5 & 14 & 906 & 5 & 12 \\
\hline 9 & 1,050 & 16 & 45 & 1,020 & 6 & 17 & 895 & 5 & 12 \\
\hline 10 & 1,090 & 18 & 53 & 1,020 & 7 & 19 & 831 & 5 & 11 \\
\hline 11 & 1,150 & 19 & 59 & 1,010 & 8 & 22 & 828 & 7 & 16 \\
\hline 12 & 1,130 & 18 & 55 & 1,000 & 10 & 27 & 963 & 8 & 21 \\
\hline 13 & 1,110 & 18 & 54 & 1,050 & 13 & 37 & 963 & 8 & 21 \\
\hline 14 & 1,110 & 16 & 48 & 1,100 & 14 & 42 & 973 & 8 & 21 \\
\hline 15 & 1,130 & 14 & 43 & 1,120 & 13 & 39 & 955 & 8 & 21 \\
\hline 16 & 1,150 & 12 & 37 & 1,110 & 10 & 30 & 927 & 8 & 20 \\
\hline 17 & 1,120 & 10 & 30 & 1,090 & 9 & 26 & 943 & 8 & 20 \\
\hline 18 & 1,100 & 9 & 27 & 1,080 & 9 & 26 & 1,000 & 8 & 22 \\
\hline 19 & 1,090 & 8 & 24 & 1,080 & 9 & 26 & 743 & 8 & 16 \\
\hline 20 & 1,090 & 8 & 24 & 1,060 & 9 & 26 & 350 & 9 & 8.5 \\
\hline 21 & 1,100 & 8 & 24 & 1,070 & 10 & 29 & 200 & 10 & 5.4 \\
\hline 22 & 1,090 & 9 & 26 & 1,250 & 16 & 54 & 300 & 12 & 9.7 \\
\hline 23 & 1,080 & 9 & 26 & 1,230 & 14 & 46 & 400 & 14 & 15 \\
\hline 24 & 1,080 & 8 & 23 & 1,200 & 11 & 36 & 500 & 10 & 14 \\
\hline 25 & 1,060 & 8 & 23 & 1,170 & 10 & 32 & 600 & 8 & 13 \\
\hline 26 & 1,050 & 8 & 23 & 1,150 & 9 & 28 & 700 & 56 & 106 \\
\hline 27 & 1,040 & 8 & 22 & 1,130 & 9 & 27 & 750 & 52 & 105 \\
\hline 28 & 1,030 & 7 & 19 & 1,110 & 9 & 27 & 800 & 50 & 108 \\
\hline 29 & 1,010 & 5 & 14 & 1,090 & 8 & 24 & 850 & 50 & 115 \\
\hline 30 & 1,010 & 5 & 14 & 1,080 & 8 & 23 & 950 & 37 & 95 \\
\hline 31 & 1,000 & 5 & 14 & -. & -- & - & 1,060 & 27 & 77 \\
\hline TOTAL & 33,107 & --- & 982 & 32,197 & - & 754.2 & 25,685 & -- & $1,009.6$ \\
\hline MEAN & 1,068 & 11 & 32 & 1,073 & 8 & 25 & 829 & 15 & 33 \\
\hline MAX & 1,150 & 19 & 59 & 1,250 & 16 & 54 & 1,130 & 56 & 115 \\
\hline MIN & 955 & 5 & 14 & 989 & 3 & 8.1 & 200 & 4 & 54 \\
\hline
\end{tabular}


Table 6. Daily streamflow and suspended-sediment data for Clark Fork at Turah Bridge, near Bonner, Montana, October 1998 through September 1999 (Continued)

\begin{tabular}{|c|c|c|c|c|c|c|c|c|c|}
\hline \multirow[b]{2}{*}{ Day } & \multirow{2}{*}{$\begin{array}{c}\text { Mean } \\
\text { stream- } \\
\text { flow } \\
\left(\mathbf{f t}^{3} / \mathbf{s}\right)\end{array}$} & \multicolumn{2}{|c|}{ Suspended sediment } & \multirow{2}{*}{$\begin{array}{c}\text { Mean } \\
\text { stream- } \\
\text { flow } \\
\left(\mathbf{f t}^{\mathbf{3}} / \mathbf{s}\right)\end{array}$} & \multicolumn{2}{|c|}{ Suspended sediment } & \multirow{2}{*}{$\begin{array}{c}\text { Mean } \\
\text { stream- } \\
\text { flow } \\
\left(\mathrm{ft}^{3} / \mathrm{s}\right)\end{array}$} & \multicolumn{2}{|c|}{ Suspended sediment } \\
\hline & & $\begin{array}{c}\text { Mean } \\
\text { concen- } \\
\text { tration } \\
(m g / L)\end{array}$ & $\begin{array}{c}\text { Dis- } \\
\text { charge } \\
\text { (ton/d) }\end{array}$ & & $\begin{array}{c}\text { Mean } \\
\text { concen- } \\
\text { tration } \\
(\mathrm{mg} / \mathrm{L})\end{array}$ & $\begin{array}{l}\text { Dis- } \\
\text { charge } \\
\text { (ton/d) }\end{array}$ & & $\begin{array}{l}\text { Mean } \\
\text { concen- } \\
\text { tration } \\
\text { (mg/L) }\end{array}$ & $\begin{array}{l}\text { Dis- } \\
\text { charge } \\
\text { (ton/d) }\end{array}$ \\
\hline \multicolumn{10}{|c|}{1999} \\
\hline & \multicolumn{3}{|c|}{ January } & \multicolumn{3}{|c|}{ February } & \multicolumn{3}{|c|}{ March } \\
\hline 1 & 1,010 & 10 & 27 & 800 & 12 & 26 & 1,430 & 207 & 799 \\
\hline 2 & 963 & 8 & 21 & 807 & 10 & 22 & 1,280 & 75 & 259 \\
\hline 3 & 923 & 8 & 20 & 806 & 10 & 22 & 1,120 & 30 & 91 \\
\hline 4 & 850 & 8 & 18 & 817 & 9 & 20 & 1,020 & 19 & 52 \\
\hline 5 & 938 & 8 & 20 & 796 & 9 & 19 & 917 & 13 & 32 \\
\hline 6 & 996 & 8 & 22 & 794 & 9 & 19 & 884 & 10 & 24 \\
\hline 7 & 1,020 & 9 & 25 & 818 & 9 & 20 & 842 & 10 & 23 \\
\hline 8 & 990 & 10 & 27 & 817 & 9 & 20 & 875 & 11 & 26 \\
\hline 9 & 959 & 15 & 39 & 812 & 9 & 20 & 880 & 12 & 29 \\
\hline 10 & 957 & 21 & 54 & 750 & 8 & 16 & 888 & 11 & 26 \\
\hline 11 & 1,010 & 26 & 71 & 700 & 8 & 15 & 885 & 10 & 24 \\
\hline 12 & 1,080 & 28 & 82 & 707 & 7 & 13 & 868 & 10 & 23 \\
\hline 13 & 1,020 & 26 & 72 & 725 & 6 & 12 & 869 & 12 & 28 \\
\hline 14 & 963 & 24 & 62 & 807 & 5 & II & 957 & 18 & 47 \\
\hline 15 & 1,000 & 22 & 59 & 813 & 5 & 11 & 1,130 & 30 & 92 \\
\hline 16 & 1,040 & 20 & 56 & 771 & 6 & 12 & 1,330 & 50 & 180 \\
\hline 17 & 972 & 19 & 50 & 794 & 8 & 17 & 1,240 & 37 & 124 \\
\hline 18 & 940 & 17 & 43 & 789 & 8 & 17 & 1,130 & 23 & 70 \\
\hline 19 & 927 & 15 & 38 & 776 & 6 & 13 & 1,190 & 30 & 96 \\
\hline 20 & 915 & 14 & 35 & 775 & 5 & 10 & 1,370 & 46 & 170 \\
\hline 21 & 907 & 12 & 29 & 760 & 5 & 10 & 1,560 & 67 & 282 \\
\hline 22 & 885 & 11 & 26 & 762 & 5 & 10 & 1,770 & 100 & 478 \\
\hline 23 & 850 & 10 & 23 & 798 & 5 & 11 & 1,790 & 84 & 406 \\
\hline 24 & 750 & 9 & 18 & 811 & 5 & 11 & 1,720 & 62 & 288 \\
\hline 25 & 700 & 8 & 15 & 949 & 22 & 56 & 1,760 & 60 & 285 \\
\hline 26 & 750 & 7 & 14 & 1,010 & 25 & 68 & 1,880 & 76 & 386 \\
\hline 27 & 819 & 8 & 18 & 930 & 20 & 50 & 1,860 & 60 & 301 \\
\hline 28 & 763 & 10 & 21 & 911 & 28 & 69 & 1.660 & 33 & 148 \\
\hline 29 & 794 & 12 & 26 & - & -- & --- & 1,540 & 30 & 125 \\
\hline 30 & 785 & 12 & 25 & -- & -- & -- & 1,500 & 24 & 97 \\
\hline 31 & 807 & 13 & 28 & - & - & - & 1,430 & 21 & 81 \\
\hline TOTAL & 28,283 & --- & 1,084 & 22,605 & -- & 620 & 39,575 & -- & 5,092 \\
\hline MEAN & 912 & 14 & 35 & 807 & 10 & 22 & 1,277 & 41 & 164 \\
\hline MAX & 1,080 & 28 & 82 & 1,010 & 28 & 69 & 1,880 & 207 & 799 \\
\hline MIN & 700 & 7 & 14 & 700 & 5 & 10 & 842 & 10 & 23 \\
\hline
\end{tabular}


Table 6. Daily streamflow and suspended-sediment data for Clark Fork at Turah Bridge, near Bonner, Montana, October 1998 through September 1999 (Continued)

\begin{tabular}{|c|c|c|c|c|c|c|c|c|c|}
\hline \multirow[b]{2}{*}{ Day } & \multirow{2}{*}{$\begin{array}{c}\text { Mean } \\
\text { stream- } \\
\text { flow } \\
\left(\mathrm{ft}^{3} / \mathrm{s}\right)\end{array}$} & \multicolumn{2}{|c|}{ Suspended sediment } & \multirow{2}{*}{$\begin{array}{c}\text { Mean } \\
\text { stream- } \\
\text { flow } \\
\left(\mathrm{ft}^{3} / \mathrm{s}\right)\end{array}$} & \multicolumn{2}{|c|}{ Suspended sediment } & \multirow{2}{*}{$\begin{array}{c}\text { Mean } \\
\text { stream- } \\
\text { flow } \\
\left(\mathbf{f t}^{\mathbf{3}} / \mathbf{s}\right)\end{array}$} & \multicolumn{2}{|c|}{ Suspended sediment } \\
\hline & & $\begin{array}{l}\text { Mean } \\
\text { concen- } \\
\text { tration } \\
(\mathrm{mg} / \mathrm{L})\end{array}$ & $\begin{array}{c}\text { Dis- } \\
\text { charge } \\
\text { (ton/d) }\end{array}$ & & $\begin{array}{l}\text { Mean } \\
\text { concen- } \\
\text { tration } \\
(\mathrm{mg} / \mathrm{L})\end{array}$ & $\begin{array}{l}\text { Dis- } \\
\text { charge } \\
\text { (ton/d) }\end{array}$ & & $\begin{array}{l}\text { Mean } \\
\text { concen- } \\
\text { tration } \\
(m g / L)\end{array}$ & $\begin{array}{l}\text { Dis- } \\
\text { charge } \\
\text { (ton/d) }\end{array}$ \\
\hline \multicolumn{10}{|c|}{1999} \\
\hline & \multicolumn{3}{|c|}{ April } & \multicolumn{3}{|c|}{ May } & \multicolumn{3}{|c|}{ June } \\
\hline 1 & 1,360 & 20 & 73 & 1,890 & 33 & 168 & 4,570 & 82 & 1,010 \\
\hline 2 & 1,290 & 17 & 59 & 1,940 & 32 & 168 & 4,630 & 75 & 938 \\
\hline 3 & 1,250 & 17 & 57 & 1,990 & 32 & 172 & 5,050 & 98 & 1,340 \\
\hline 4 & 1,240 & 17 & 57 & 1,990 & 30 & 161 & 6,340 & 212 & 3.630 \\
\hline 5 & 1,210 & 14 & 46 & 1,920 & 28 & 145 & 6,440 & 166 & 2,890 \\
\hline 6 & 1,170 & 12 & 38 & 1,830 & 22 & 109 & 5,930 & 123 & 1,970 \\
\hline 7 & 1,140 & 10 & 31 & 1,800 & 21 & 102 & 5,310 & 84 & 1,200 \\
\hline 8 & 1,140 & 11 & 34 & 1,820 & 18 & 88 & 4,810 & 64 & 831 \\
\hline 9 & 1,160 & 11 & 34 & 1,830 & 19 & 94 & 4,510 & 59 & 718 \\
\hline 10 & 1,170 & 10 & 32 & 1,840 & 18 & 89 & 4,020 & 48 & 521 \\
\hline II & 1,180 & 9 & 29 & 1,820 & 18 & 88 & 3,530 & 44 & 419 \\
\hline 12 & 1,190 & 8 & 26 & 1,790 & 19 & 92 & 3,210 & 40 & 347 \\
\hline 13 & 1,200 & 10 & 32 & 1,770 & 18 & 86 & 3,070 & 37 & 307 \\
\hline 14 & 1,210 & 12 & 39 & 1,710 & 13 & 60 & 3,180 & 39 & 335 \\
\hline 15 & 1,230 & 11 & 37 & 1,610 & 11 & 48 & 3,420 & 40 & 369 \\
\hline 16 & 1,230 & 12 & 40 & 1,560 & 10 & 42 & 3,750 & 50 & 506 \\
\hline 17 & 1,250 & 16 & 54 & 1,570 & II & 47 & 4,240 & 72 & 824 \\
\hline 18 & 1,340 & 18 & 65 & 1,590 & 11 & 47 & 4,260 & 65 & 748 \\
\hline 19 & 1,520 & 34 & 140 & 1,780 & 16 & 77 & 4,170 & 59 & 664 \\
\hline 20 & 1,790 & 56 & 271 & 1,980 & 26 & 139 & 4,020 & 51 & 554 \\
\hline 21 & 1,930 & 58 & 302 & 2,120 & 39 & 223 & 3,740 & 42 & 424 \\
\hline 22 & 1,880 & 43 & 218 & 2,380 & 47 & 302 & 3,520 & 29 & 276 \\
\hline 23 & 1,780 & 35 & 168 & 2,610 & 55 & 388 & 3,390 & 26 & 238 \\
\hline 24 & 1,720 & 28 & 130 & 2,900 & 81 & 634 & 3,070 & 27 & 224 \\
\hline 25 & 1,720 & 27 & 125 & 3,430 & 116 & 1,070 & 2,960 & 25 & 200 \\
\hline 26 & 1,820 & 31 & 152 & 4,130 & 178 & 1,980 & 2,870 & 21 & 163 \\
\hline 27 & 1,960 & 45 & 238 & 4,540 & 148 & 1,810 & 2,630 & 20 & 142 \\
\hline 28 & 2,070 & 57 & 319 & 4,440 & 107 & 1,280 & 2,430 & 16 & 105 \\
\hline 29 & 2,040 & 50 & 275 & 4,610 & 110 & 1,370 & 2,210 & 12 & 72 \\
\hline 30 & 1,950 & 34 & 179 & 5,390 & 186 & 2,710 & 2,110 & 13 & 74 \\
\hline 31 & -- & - & -- & 5,160 & 138 & 1,920 & -- & - & -- \\
\hline TOTAL & 44,140 & - & 3,300 & 77,740 & --- & 15,709 & 117,390 & - & 22,039 \\
\hline MEAN & 1,471 & 24 & 110 & 2,508 & 52 & 507 & 3,913 & 58 & 735 \\
\hline MAX & 2,070 & 58 & 319 & 5,390 & 186 & 2,710 & 6,440 & 212 & 3,630 \\
\hline MIN & 1,140 & 8 & 26 & 1,560 & 10 & 42 & 2,110 & 12 & 72 \\
\hline
\end{tabular}


Table 6. Daily streamflow and suspended-sediment data for Clark Fork at Turah Bridge, near Bonner, Montana, October 1998 through September 1999 (Continued)

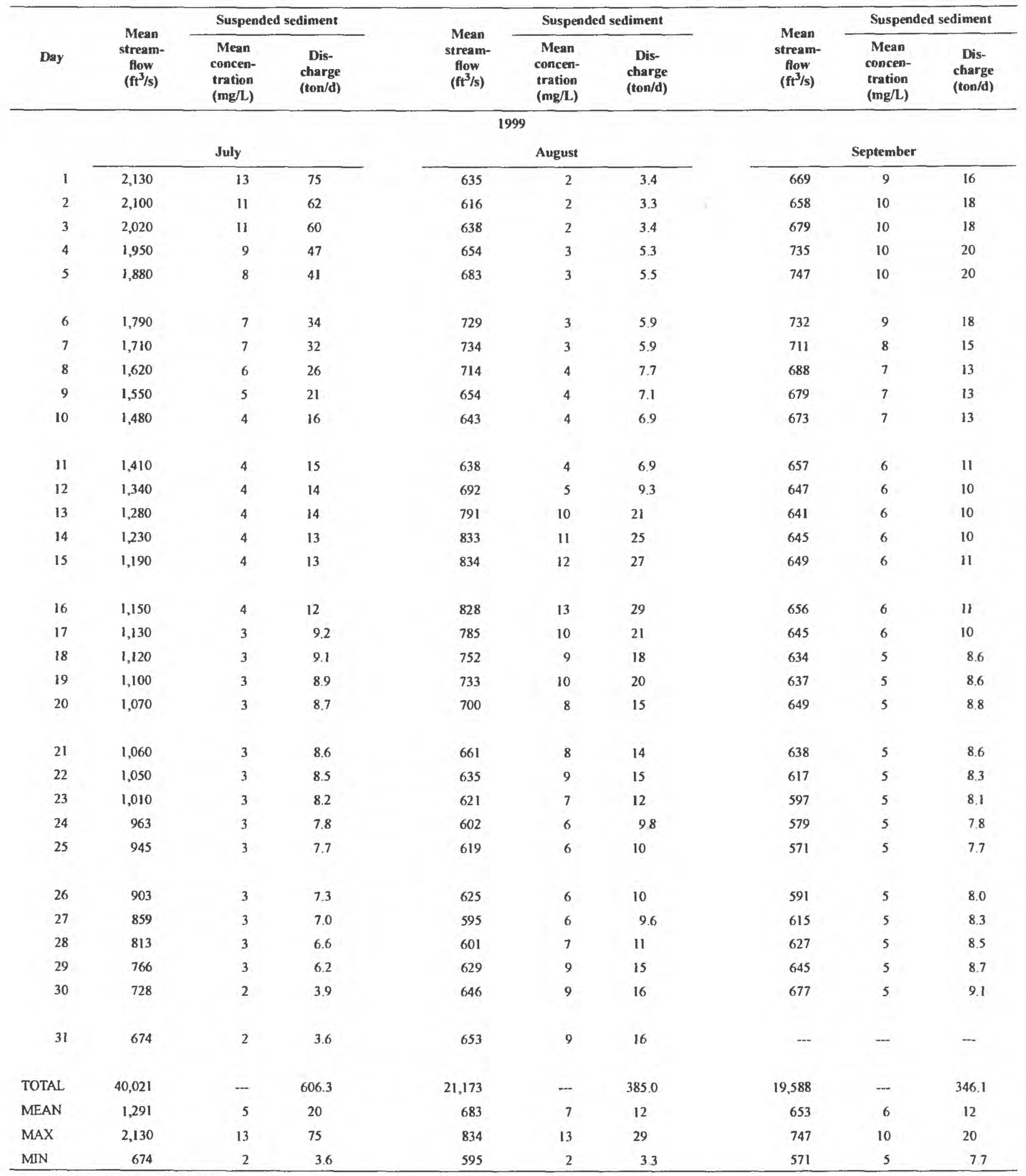

TOTAL FOR WATER YEAR 1999

STREAMFLOW--501, $504 \mathrm{ft}^{3} / \mathrm{s}$

SEDIMENT DISCHARGE--51,927.2 tons

42 Water-quality, bed-sediment, and biological data (October 1998 through September 1999) and statistical summaries of data for streams in the Upper Clark Fork Basin, Montana 
Table 7. Daily streamflow and suspended-sediment data for Clark Fork above Missoula, Montana, October 1998 through September 1999 [Abbreviations: $\mathrm{ft}^{3} / \mathrm{s}$, cubic feet per second; e, estimated; $\mathrm{mg} / \mathrm{L}$, milligrams per liter; ton/d, tons per day. Symbol: ---, no data]

\begin{tabular}{|c|c|c|c|c|c|c|c|c|c|}
\hline \multirow[b]{2}{*}{ Day } & \multirow[b]{2}{*}{$\begin{array}{c}\text { Mean } \\
\text { stream- } \\
\text { flow } \\
\left(\mathrm{fi}^{3} / \mathrm{s}\right)\end{array}$} & \multicolumn{2}{|c|}{ Suspended sediment } & \multirow[b]{2}{*}{$\begin{array}{c}\text { Mean } \\
\text { stream- } \\
\text { flow } \\
\left(\mathrm{ft}^{3} / \mathrm{s}\right)\end{array}$} & \multicolumn{2}{|c|}{ Suspended sediment } & \multirow[b]{2}{*}{$\begin{array}{c}\text { Mean } \\
\text { stream- } \\
\text { flow } \\
\left(\mathrm{ft}^{3} / \mathrm{s}\right)\end{array}$} & \multicolumn{2}{|c|}{ Suspended sediment } \\
\hline & & $\begin{array}{l}\text { Mean } \\
\text { concen- } \\
\text { tration } \\
(\mathrm{mg} / \mathrm{L})\end{array}$ & $\begin{array}{l}\text { Dis- } \\
\text { charge } \\
\text { (ton/d) }\end{array}$ & & $\begin{array}{l}\text { Mean } \\
\text { concen- } \\
\text { tration } \\
(\mathrm{mg} / \mathrm{L})\end{array}$ & $\begin{array}{l}\text { Dis- } \\
\text { charge } \\
\text { (ton/d) }\end{array}$ & & $\begin{array}{c}\text { Mean } \\
\text { concen- } \\
\text { tration } \\
(\mathrm{mg} / \mathrm{L})\end{array}$ & $\begin{array}{l}\text { Dis- } \\
\text { charge } \\
\text { (ton/d) }\end{array}$ \\
\hline \multicolumn{10}{|c|}{1998} \\
\hline & \multicolumn{3}{|c|}{ October } & \multicolumn{3}{|c|}{ November } & \multicolumn{3}{|c|}{ December } \\
\hline 1 & 1,460 & 6 & 24 & 1,540 & 4 & 17 & 1,770 & 5 & 24 \\
\hline 2 & 1,500 & 6 & 24 & 1,540 & 4 & 17 & 1,790 & 5 & 24 \\
\hline 3 & 1,540 & 5 & 21 & 1,560 & 4 & 17 & 1,850 & 5 & 25 \\
\hline 4 & 1,570 & 5 & 21 & 1,550 & 3 & 13 & 1,830 & 4 & 20 \\
\hline 5 & 1,600 & 5 & 22 & 1,530 & 3 & 12 & 1,760 & 4 & 19 \\
\hline 6 & 1,580 & 4 & 17 & 1,560 & 3 & 13 & 1,590 & 5 & 21 \\
\hline 7 & 1,570 & 5 & 21 & 1,560 & 3 & 13 & 1,550 & 5 & 21 \\
\hline 8 & 1,560 & 5 & 21 & 1,580 & 3 & 13 & 1,560 & 5 & 21 \\
\hline 9 & 1,560 & 5 & 21 & 1,570 & 3 & 13 & 1,520 & 4 & 16 \\
\hline 10 & 1,600 & 6 & 26 & 1,540 & 4 & 17 & 1,420 & 4 & 15 \\
\hline 11 & 1,690 & 6 & 27 & 1,560 & 5 & 21 & 1,430 & 5 & 19 \\
\hline 12 & 1,710 & 6 & 28 & 1,520 & 5 & 21 & 1,560 & 6 & 25 \\
\hline 13 & 1,670 & 7 & 32 & 1,580 & 6 & 26 & 1,570 & 7 & 30 \\
\hline 14 & 1,680 & 7 & 32 & 1,700 & 6 & 28 & 1,570 & 6 & 25 \\
\hline 15 & 1,670 & 7 & 32 & 1,780 & 6 & 29 & 1,550 & 6 & 25 \\
\hline 16 & 1,680 & 7 & 32 & 1,780 & 6 & 29 & 1,500 & 5 & 20 \\
\hline 17 & 1,680 & 7 & 32 & 1,730 & 5 & 23 & 1,530 & 5 & 21 \\
\hline 18 & 1,670 & 6 & 27 & 1,720 & 5 & 23 & 1,520 & 4 & 16 \\
\hline 19 & 1,640 & 6 & 27 & 1,680 & 5 & 23 & e 1,050 & 4 & 11 \\
\hline 20 & 1,620 & 5 & 22 & 1,660 & 5 & 22 & e 600 & 4 & 6.5 \\
\hline 21 & 1,610 & 5 & 22 & 1,660 & 5 & 22 & es00 & 4 & 5.4 \\
\hline 22 & 1,610 & 5 & 22 & 1,880 & 6 & 30 & e 450 & 3 & 3.6 \\
\hline 23 & 1,600 & 5 & 22 & 1,940 & 6 & 31 & e550 & 3 & 4.5 \\
\hline 24 & 1,610 & 5 & 22 & 1,920 & 5 & 26 & e700 & 3 & 5.7 \\
\hline 25 & 1,600 & 5 & 22 & 1,850 & 5 & 25 & e900 & 4 & 9.7 \\
\hline 26 & 1,590 & 5 & 21 & 1,800 & 5 & 24 & e 1,100 & 7 & 21 \\
\hline 27 & 1,580 & 5 & 21 & 1,780 & 5 & 24 & el, 300 & 10 & 35 \\
\hline 28 & 1,580 & 5 & 21 & 1,820 & 6 & 29 & el, 400 & 9 & 34 \\
\hline 29 & 1,570 & 5 & 21 & 1,830 & 6 & 30 & $e 1,600$ & 8 & 35 \\
\hline 30 & 1,560 & 4 & 17 & 1,790 & 6 & 29 & el, 700 & 6 & 28 \\
\hline 31 & 1,560 & 4 & 17 & -- & - & -- & e 1,800 & 6 & 29 \\
\hline TOTAL & 49,720 & - & 737 & 50,510 & - & 660 & 42,520 & -- & 6154 \\
\hline MEAN & 1,604 & 5 & 24 & 1,684 & 5 & 22 & 1,372 & 5 & 20 \\
\hline MAX & 1,710 & 7 & 32 & 1,940 & 6 & 31 & 1,850 & 10 & 35 \\
\hline MIN & 1,460 & 4 & 17 & 1,520 & 3 & 12 & 450 & 3 & 36 \\
\hline
\end{tabular}


Table 7. Daily streamflow and suspended-sediment data for Clark Fork above Missoula. Montana. October 1998 through September 1999 (Continued)

\begin{tabular}{|c|c|c|c|c|c|c|c|c|c|}
\hline \multirow[b]{2}{*}{ Day } & \multirow[b]{2}{*}{$\begin{array}{c}\text { Mean } \\
\text { stream- } \\
\text { flow } \\
\left(\mathrm{ft}^{3} / \mathrm{s}\right)\end{array}$} & \multicolumn{2}{|c|}{ Suspended sediment } & \multirow[b]{2}{*}{$\begin{array}{c}\text { Mean } \\
\text { stream- } \\
\text { flow } \\
\left(\mathbf{f t}^{3} / \mathrm{s}\right)\end{array}$} & \multicolumn{2}{|c|}{ Suspended sediment } & \multirow[b]{2}{*}{$\begin{array}{c}\text { Mean } \\
\text { stream- } \\
\text { flow } \\
\left(\mathrm{ft}^{3} / \mathrm{s}\right)\end{array}$} & \multicolumn{2}{|c|}{ Suspended sediment } \\
\hline & & $\begin{array}{l}\text { Mean } \\
\text { concen- } \\
\text { tration } \\
(\mathrm{mg} / \mathrm{L})\end{array}$ & $\begin{array}{c}\text { Dis- } \\
\text { charge } \\
\text { (ton/d) }\end{array}$ & & $\begin{array}{l}\text { Mean } \\
\text { concen- } \\
\text { tration } \\
(\mathrm{mg} / \mathrm{L})\end{array}$ & $\begin{array}{c}\text { Dis- } \\
\text { charge } \\
\text { (ton/d) }\end{array}$ & & $\begin{array}{l}\text { Mean } \\
\text { concen- } \\
\text { tration } \\
(\mathrm{mg} / \mathrm{L})\end{array}$ & $\begin{array}{l}\text { Dis- } \\
\text { charge } \\
\text { (ton/d) }\end{array}$ \\
\hline \multicolumn{10}{|c|}{1999} \\
\hline & \multicolumn{3}{|c|}{ January } & \multicolumn{3}{|c|}{ February } & \multicolumn{3}{|c|}{ March } \\
\hline 1 & $\mathrm{el}, 900$ & 4 & 21 & 1,430 & 4 & 15 & 1,940 & 18 & 94 \\
\hline 2 & $\mathrm{el}, 800$ & 3 & 15 & 1,380 & 4 & 15 & 1,890 & 33 & 168 \\
\hline 3 & 1,610 & 2 & 87 & 1,370 & 4 & 15 & 1,750 & 12 & 57 \\
\hline 4 & 1,520 & 3 & 12 & 1,370 & 4 & 15 & 1.680 & 7 & 32 \\
\hline 5 & 1,570 & 5 & 21 & 1,370 & 3 & 11 & 1,510 & 6 & 24 \\
\hline 6 & 1,630 & 4 & 18 & 1,330 & 4 & 14 & 1,460 & 5 & 20 \\
\hline 7 & 1,670 & 4 & 18 & 1,380 & 4 & 15 & 1,410 & 4 & 15 \\
\hline 8 & 1,610 & 4 & 17 & 1,350 & 4 & 15 & 1,450 & 7 & 27 \\
\hline 9 & 1,590 & 4 & 17 & 1,350 & 4 & 15 & 1,420 & 6 & 23 \\
\hline 10 & 1,570 & 4 & 17 & 1,350 & 4 & 15 & 1,430 & 5 & 19 \\
\hline 11 & 1,690 & 6 & 27 & 1,300 & 3 & 11 & 1,440 & 6 & 23 \\
\hline 12 & 1,680 & 8 & 36 & 1,170 & 2 & 6.3 & 1,430 & 4 & 15 \\
\hline 13 & 1,650 & 9 & 40 & 1,210 & 2 & 6.5 & 1,420 & 5 & 19 \\
\hline 14 & 1,620 & 8 & 35 & 1,340 & 3 & 11 & 1,500 & 6 & 24 \\
\hline 15 & 1,640 & 8 & 35 & 1,340 & 3 & 11 & 1,690 & 7 & 32 \\
\hline 16 & 1,680 & 8 & 36 & 1,300 & 4 & 14 & 2,000 & 10 & 54 \\
\hline 17 & 1,660 & 8 & 36 & 1,340 & 4 & 14 & 2,280 & 16 & 98 \\
\hline 18 & 1,600 & 8 & 35 & 1,320 & 4 & 14 & 1,940 & 12 & 63 \\
\hline 19 & 1,550 & 9 & 38 & 1,310 & 3 & 11 & 1,900 & 11 & 56 \\
\hline 20 & 1,540 & 9 & 37 & 1,320 & 3 & 11 & 2,120 & 17 & 97 \\
\hline 21 & 1,520 & 10 & 41 & 1,300 & 3 & 11 & 2,650 & 17 & 122 \\
\hline 22 & 1,470 & 9 & 36 & 1,310 & 3 & 11 & 3,150 & 27 & 230 \\
\hline 23 & 1,460 & 8 & 32 & 1,360 & 3 & 11 & 3,330 & 29 & 261 \\
\hline 24 & 1,350 & 6 & 22 & 1,330 & 3 & 11 & 3,210 & 21 & 182 \\
\hline 25 & 1,180 & 4 & 13 & 1,470 & 3 & 12 & 3,230 & 17 & 148 \\
\hline 26 & 1,190 & 4 & 13 & 1,550 & 4 & 17 & 3,630 & 23 & 225 \\
\hline 27 & 1,330 & 4 & 14 & 1,460 & 6 & 24 & 3,720 & 24 & $24 \mathrm{~J}$ \\
\hline 28 & 1,450 & 4 & 16 & 1,500 & 7 & 28 & 3,390 & 17 & 156 \\
\hline 29 & 1,430 & 3 & 12 & -- & -- & -- & 3,190 & 14 & 121 \\
\hline 30 & 1,550 & 4 & 17 & - & - & -- & 3,050 & 14 & 115 \\
\hline 31 & 1,430 & 4 & 15 & $\ldots$ & $\ldots$ & --- & 2,970 & 14 & 112 \\
\hline TOTAL & 48,140 & -- & 750.7 & 37,910 & -- & 379.8 & 69,180 & -- & 2,873 \\
\hline MEAN & 1,553 & 6 & 24 & 1,354 & 4 & 14 & 2,232 & 13 & 93 \\
\hline MAX & 1,900 & 10 & 41 & 1,550 & 7 & 28 & 3,720 & 33 & 261 \\
\hline MIN & 1,180 & 2 & 8.7 & 1,170 & 2 & 6.3 & 1,410 & 4 & 15 \\
\hline
\end{tabular}


Table 7. Daily streamflow and suspended-sediment data for Clark Fork above Missoula, Montana, October 1998 through September 1999 (Continued)

\begin{tabular}{|c|c|c|c|c|c|c|c|c|c|}
\hline \multirow[b]{2}{*}{ Day } & \multirow[b]{2}{*}{$\begin{array}{c}\text { Mean } \\
\text { stream- } \\
\text { flow } \\
\left(\mathrm{ft}^{3} / \mathrm{s}\right)\end{array}$} & \multicolumn{2}{|c|}{ Suspended sediment } & \multirow[b]{2}{*}{$\begin{array}{c}\text { Mean } \\
\text { stream- } \\
\text { flow } \\
\left(\mathrm{ft}^{3} / \mathrm{s}\right)\end{array}$} & \multicolumn{2}{|c|}{ Suspended sediment } & \multirow{2}{*}{$\begin{array}{c}\text { Mean } \\
\text { stream- } \\
\text { flow } \\
\left(\mathrm{ft}^{3} / \mathrm{s}\right)\end{array}$} & \multicolumn{2}{|c|}{ Suspended sediment } \\
\hline & & $\begin{array}{l}\text { Mean } \\
\text { concen- } \\
\text { tration } \\
(\mathrm{mg} / \mathrm{L})\end{array}$ & $\begin{array}{c}\text { Dis- } \\
\text { charge } \\
\text { (ton/d) }\end{array}$ & & $\begin{array}{c}\text { Mean } \\
\text { concen- } \\
\text { tration } \\
(m g / L)\end{array}$ & $\begin{array}{c}\text { Dis- } \\
\text { charge } \\
\text { (ton/d) }\end{array}$ & & $\begin{array}{l}\text { Mean } \\
\text { concen- } \\
\text { tration } \\
(\mathrm{mg} / \mathrm{L})\end{array}$ & $\begin{array}{l}\text { Dis- } \\
\text { charge } \\
\text { (ton/d) }\end{array}$ \\
\hline \multicolumn{10}{|c|}{1999} \\
\hline & \multicolumn{3}{|c|}{ April } & \multicolumn{3}{|c|}{ May } & \multicolumn{3}{|c|}{ June } \\
\hline 1 & 2,810 & 12 & 91 & 5,340 & 19 & 274 & 11,500 & 63 & 1,960 \\
\hline 2 & 2,620 & 10 & 71 & 5,340 & 18 & 260 & 11,200 & 59 & 1,780 \\
\hline 3 & 2,570 & 10 & 69 & 5,460 & 18 & 265 & 11,900 & 65 & 2,090 \\
\hline 4 & 2,510 & 9 & 61 & 5,430 & 17 & 249 & 14,000 & 118 & 4,460 \\
\hline 5 & 2,430 & 7 & 46 & 5,250 & 16 & 227 & 14,400 & 114 & 4,430 \\
\hline 6 & 2,340 & 7 & 44 & 4,950 & 14 & 187 & 13,500 & 90 & 3,280 \\
\hline 7 & 2,350 & 7 & 44 & 4,860 & 14 & 184 & 12,300 & 68 & 2,260 \\
\hline 8 & 2,370 & 8 & 51 & 4,880 & 10 & 132 & 11,100 & 50 & 1,500 \\
\hline 9 & 2,470 & 7 & 47 & 4,900 & 12 & 159 & 10,300 & 43 & 1,200 \\
\hline 10 & 2,430 & 6 & 39 & 4,890 & 12 & 158 & 9,300 & 39 & 979 \\
\hline 11 & 2,320 & 6 & 38 & 4,790 & 12 & 155 & 8,400 & 32 & 726 \\
\hline 12 & 2,380 & 7 & 45 & 4,640 & 13 & 163 & 7,790 & 27 & 568 \\
\hline 13 & 2,450 & 8 & 53 & 4,560 & 13 & 160 & 7,560 & 24 & 490 \\
\hline 14 & 2,570 & 8 & 56 & 4,380 & 12 & 142 & 7,870 & 25 & 531 \\
\hline 15 & 2,650 & 8 & 57 & 4,210 & 10 & 114 & 8,760 & 32 & 757 \\
\hline 16 & 2,640 & 8 & 57 & 4,150 & 11 & 123 & 9,530 & 40 & 1,030 \\
\hline 17 & 2,760 & 9 & 67 & 4,160 & 10 & 112 & 10,400 & 48 & 1,350 \\
\hline 18 & 3,010 & 10 & 81 & 4,330 & 10 & 117 & 10,500 & 50 & 1,420 \\
\hline 19 & 3,520 & 13 & 124 & 4,840 & 12 & 157 & 10,400 & 49 & 1,380 \\
\hline 20 & 4,420 & 18 & 215 & 5,540 & 18 & 269 & 10,200 & 41 & 1,130 \\
\hline 21 & 4,940 & 23 & 307 & 6,210 & 29 & 486 & 9,510 & 36 & 924 \\
\hline 22 & 4,900 & 21 & 278 & 7,230 & 41 & 800 & 8,940 & 29 & 700 \\
\hline 23 & 4,720 & 17 & 217 & 7,960 & 53 & 1,140 & 8,590 & 26 & 603 \\
\hline 24 & 4,610 & 16 & 199 & 9,020 & 65 & 1,580 & 7,910 & 25 & 534 \\
\hline 25 & 4,710 & 17 & 216 & 10,800 & 110 & 3,210 & 7,770 & 22 & 462 \\
\hline 26 & 5,120 & 20 & 276 & 13,000 & 162 & 5,690 & 7,650 & 22 & 454 \\
\hline 27 & 5,710 & 23 & 355 & 13,500 & 140 & 5,100 & 6,900 & 20 & 373 \\
\hline 28 & 6,110 & 26 & 429 & 13,100 & 108 & 3,820 & 6,270 & 16 & 271 \\
\hline 29 & 5,970 & 24 & 387 & 13,000 & 93 & 3,260 & 5,820 & 12 & 189 \\
\hline 30 & 5,600 & 20 & 302 & 13,500 & 105 & 3,830 & 5,620 & 14 & 212 \\
\hline 31 & - & - & $\cdots$ & 12,700 & 96 & 3,290 & - & -- & - \\
\hline TOTAL & 106,010 & $\cdots$ & 4,322 & 216,920 & - & 35,813 & 285,890 & -- & 38,043 \\
\hline MEAN & 3,534 & 13 & 144 & 6,997 & 41 & 1,160 & 9,530 & 43 & 1.270 \\
\hline MAX & 6,110 & 26 & 429 & 13,500 & 162 & 5,690 & 14,400 & 118 & 4,460 \\
\hline MIN & 2,320 & 6 & 38 & 4,150 & 10 & 112 & 5,620 & 12 & 189 \\
\hline
\end{tabular}


Table 7. Daily streamflow and suspended-sediment data for Clark Fork above Missoula, Montana, October 1998 through September 1999 (Continued)

\begin{tabular}{|c|c|c|c|c|c|c|c|c|c|}
\hline \multirow[b]{2}{*}{ Day } & \multirow[b]{2}{*}{$\begin{array}{c}\text { Mean } \\
\text { stream- } \\
\text { flow } \\
\left(\mathrm{ft}^{3} / \mathrm{s}\right)\end{array}$} & \multicolumn{2}{|c|}{ Suspended sediment } & \multirow[b]{2}{*}{$\begin{array}{c}\text { Mean } \\
\text { stream- } \\
\text { flow } \\
\left(\mathrm{ft}^{3} / \mathrm{s}\right)\end{array}$} & \multicolumn{2}{|c|}{ Suspended sediment } & \multirow[b]{2}{*}{$\begin{array}{c}\text { Mean } \\
\text { stream- } \\
\text { flow } \\
\left(\mathrm{ft}^{3} / \mathrm{s}\right)\end{array}$} & \multicolumn{2}{|c|}{ Suspended sediment } \\
\hline & & $\begin{array}{l}\text { Mean } \\
\text { concen- } \\
\text { tration } \\
(\mathrm{mg} / \mathrm{L})\end{array}$ & $\begin{array}{l}\text { Dis- } \\
\text { charge } \\
\text { (ton/d) }\end{array}$ & & $\begin{array}{l}\text { Mean } \\
\text { concen- } \\
\text { tration } \\
(\mathrm{mg} / \mathrm{L})\end{array}$ & $\begin{array}{c}\text { Dis- } \\
\text { charge } \\
\text { (ton/d) }\end{array}$ & & $\begin{array}{c}\text { Mean } \\
\text { concen- } \\
\text { tration } \\
(\mathrm{mg} / \mathrm{L})\end{array}$ & $\begin{array}{l}\text { Dis- } \\
\text { charge } \\
\text { (ton/d) }\end{array}$ \\
\hline \multicolumn{10}{|c|}{1999} \\
\hline & \multicolumn{3}{|c|}{ July } & \multicolumn{3}{|c|}{ August } & \multicolumn{3}{|c|}{ September } \\
\hline 1 & 5,890 & 14 & 223 & 1,730 & 6 & 28 & 1.420 & 6 & 23 \\
\hline 2 & 5,900 & 14 & 223 & 1,710 & 6 & 28 & 1,400 & 6 & 23 \\
\hline 3 & 5,610 & 14 & 212 & 1,690 & 7 & 32 & 1,420 & 7 & 27 \\
\hline 4 & 5,290 & 12 & 171 & 1,680 & 7 & 32 & 1,500 & 7 & 28 \\
\hline 5 & 5,030 & 12 & 163 & 1,680 & 7 & 32 & 1,530 & 8 & 33 \\
\hline 6 & 4,710 & 10 & 127 & 1,710 & 8 & 37 & 1,530 & 8 & 33 \\
\hline 7 & 4,500 & 7 & 85 & 1,740 & 8 & 38 & 1,480 & 8 & 32 \\
\hline 8 & 4,290 & 6 & 69 & 1,730 & 8 & 37 & 1,360 & 7 & 26 \\
\hline 9 & 4,100 & 8 & 89 & 1,610 & 8 & 35 & 1,380 & 6 & 22 \\
\hline 10 & 3,950 & 9 & 96 & 1,580 & 8 & 34 & 1,340 & 6 & 22 \\
\hline 11 & 3,710 & 10 & 100 & 1,550 & 8 & 33 & 1,350 & 5 & 18 \\
\hline 12 & 3,540 & 10 & 96 & 1,580 & 8 & 34 & 1,340 & 5 & 18 \\
\hline 13 & 3,430 & 11 & 102 & 1,780 & 7 & 34 & 1,290 & 5 & 17 \\
\hline 14 & 3,290 & 9 & 80 & 1,810 & 7 & 34 & 1,340 & 5 & 18 \\
\hline 15 & 3,120 & 7 & 59 & 1,820 & 8 & 39 & 1,330 & 6 & 22 \\
\hline 16 & 3,020 & 6 & 49 & 1,830 & 7 & 35 & 1,330 & 6 & 22 \\
\hline 17 & 2,930 & 6 & 47 & 1,780 & 7 & 34 & 1,300 & 6 & 21 \\
\hline 18 & 2,810 & 6 & 46 & 1,700 & 8 & 37 & 1,290 & 6 & 21 \\
\hline 19 & 2,720 & 6 & 44 & 1,630 & 9 & 40 & 1,260 & 6 & 20 \\
\hline 20 & 2,650 & 6 & 43 & 1,600 & 9 & 39 & 1,290 & 6 & 21 \\
\hline 21 & 2,530 & 6 & 41 & 1,510 & 9 & 37 & 1,300 & 7 & 25 \\
\hline 22 & 2,530 & 6 & 41 & 1,500 & 9 & 36 & 1,260 & 7 & 24 \\
\hline 23 & 2,410 & 5 & 33 & 1,450 & 12 & 47 & 1,250 & 7 & 24 \\
\hline 24 & 2,250 & 5 & 30 & 1,430 & 13 & 50 & 1,190 & 6 & 19 \\
\hline 25 & 2,260 & 5 & 31 & 1,290 & 19 & 66 & 1,200 & 6 & 19 \\
\hline 26 & 2,200 & 6 & 36 & 1,220 & 10 & 33 & 1,240 & 5 & 17 \\
\hline 27 & 2,110 & 6 & 34 & 1,250 & 7 & 24 & 1,280 & 5 & 17 \\
\hline 28 & 2,010 & 6 & 33 & 1,400 & 5 & 19 & 1,290 & 5 & 17 \\
\hline 29 & 1,940 & 6 & 31 & 1,340 & 5 & 18 & 1,290 & 5 & 17 \\
\hline 30 & 1,870 & 6 & 30 & 1,410 & 5 & 19 & 1,320 & 4 & 14 \\
\hline 31 & 1,800 & 5 & 24 & 1,380 & 6 & 22 & - & - & - \\
\hline TOTAL & 104,400 & -- & 2,488 & 49,120 & $\cdots$ & 1,063 & 40,100 & -- & 660 \\
\hline MEAN & 3,368 & 8 & 80 & 1,585 & 8 & 34 & 1,337 & 6 & 22 \\
\hline MAX & 5,900 & 14 & 223 & 1,830 & 19 & 66 & 1,530 & 8 & 33 \\
\hline $\mathrm{MIN}$ & 1,800 & 5 & 24 & 1,220 & 5 & 18 & 1,190 & 4 & 14 \\
\hline
\end{tabular}

TOTAL FOR WATER YEAR 1999 .

STREAMFLOW- $1,100,420 \mathrm{ft}^{3} / \mathrm{s}$

SEDIMENT DISCHARGE--88,404.9 tons

46 Water-quality, bed-sediment, and biological data (October 1998 through September 1999) and statistical summaries of data for streams in the Upper Clark Fork Basin, Montana 
Table 8. Chemical and suspended-sediment analyses of field replicates for water samples, upper Clark Fork basin, Montana

[Abbreviations: $\mu \mathrm{g} / \mathrm{L}$, micrograms per liter; $\mathrm{mg} / \mathrm{L}$, milligrams per liter, mm, millimeter. Symbols: <, less than laboratory reporting level]

\begin{tabular}{|c|c|c|c|c|c|c|c|c|}
\hline $\begin{array}{l}\text { Station } \\
\text { number }\end{array}$ & Station name & Date & Time & $\begin{array}{l}\text { Hardness, } \\
\text { total } \\
\left(\mathrm{mg}_{\mathrm{L}} \mathrm{L} \text { as }\right. \\
\left.\mathrm{CaCO})_{3}\right)\end{array}$ & $\begin{array}{l}\text { Calcium, } \\
\text { dissolved } \\
\text { (mg/L) }\end{array}$ & $\begin{array}{l}\text { Magnesium, } \\
\text { dissolved } \\
(\mathrm{mg} / \mathrm{L})\end{array}$ & $\begin{array}{c}\text { Arsenic, } \\
\text { total } \\
\text { recoverable } \\
(\mu \mathrm{g} / \mathrm{L})\end{array}$ & $\begin{array}{c}\text { Arsenic, } \\
\text { dissolved } \\
(\mu \mathrm{g} / \mathrm{L})\end{array}$ \\
\hline \multirow[t]{2}{*}{12323230} & \multirow{2}{*}{$\begin{array}{l}\text { Blacktail Creek at Harrison Avenue, } \\
\text { at Butte }\end{array}$} & $04-27-99$ & 1020 & 93 & 27 & 6.5 & 4 & 2 \\
\hline & & $04-27-99$ & 1025 & 94 & 27 & 6.6 & 4 & 4 \\
\hline \multirow[t]{2}{*}{12323770} & \multirow{2}{*}{ Warm Springs Creek at Warm Springs } & $06-22-99$ & 1210 & 66 & 20 & 3.6 & 5 & 4 \\
\hline & & $06-22-99$ & 1215 & 67 & 21 & 3.7 & 5 & 3 \\
\hline \multirow[t]{2}{*}{12324200} & \multirow[t]{2}{*}{ Clark Fork at Deer Lodge } & $02-22-99$ & 1540 & 230 & 67 & 15 & 9 & 7 \\
\hline & & $02-22-99$ & 1545 & 220 & 66 & 15 & 9 & 7 \\
\hline \multirow[t]{2}{*}{12331800} & \multirow[t]{2}{*}{ Clark Fork near Drummond } & 08-13-99 & 1615 & 240 & 67 & 17 & 13 & 13 \\
\hline & & $08-13-99$ & 1620 & 230 & 66 & 16 & 14 & 13 \\
\hline \multirow[t]{2}{*}{12334550} & \multirow[t]{2}{*}{ Clark Fork at Turah Bridge, near Bonner } & $05-12-99$ & 0905 & 120 & 32 & 8.6 & 6 & 5 \\
\hline & & $05-12-99$ & 0910 & 120 & 32 & 8.6 & 6 & 5 \\
\hline \multirow[t]{2}{*}{12340500} & \multirow[t]{2}{*}{ Clark Fork above Missoula } & $05-26-99$ & 1610 & 68 & 18 & 5.4 & 5 & 2 \\
\hline & & $05-26-99$ & 1615 & 68 & 18 & 5.4 & 6 & 3 \\
\hline
\end{tabular}

\begin{tabular}{|c|c|c|c|c|c|c|c|c|}
\hline $\begin{array}{c}\text { Station } \\
\text { number }\end{array}$ & Date & $\begin{array}{c}\text { Cadmium, } \\
\text { total } \\
\text { recoverable } \\
(\mu \mathrm{g} / \mathrm{L})\end{array}$ & $\begin{array}{c}\text { Cadmium, } \\
\text { dissolved } \\
(\mu \mathrm{g} / \mathrm{L})\end{array}$ & $\begin{array}{c}\text { Copper, } \\
\text { total } \\
\text { recoverable } \\
(\mu g / L)\end{array}$ & $\begin{array}{l}\text { Copper, } \\
\text { dissolved } \\
(\mu \mathrm{g} / \mathrm{L})\end{array}$ & $\begin{array}{c}\text { Iron, } \\
\text { total } \\
\text { recoverable } \\
(\mu g / L)\end{array}$ & $\begin{array}{l}\text { Iron, } \\
\text { dissolved } \\
(\mu \mathrm{g} / \mathrm{L})\end{array}$ & $\begin{array}{c}\text { Lead, } \\
\text { total } \\
\text { recoverable } \\
(\mu \mathrm{g} / \mathrm{L})\end{array}$ \\
\hline \multirow[t]{2}{*}{12323230} & $04-27-99$ & $<1$ & 0.1 & 8 & 3 & 810 & 200 & 5 \\
\hline & $04-27-99$ & $<1$ & $<.1$ & 8 & 3 & 810 & 210 & 5 \\
\hline \multirow[t]{2}{*}{12323770} & $06-22-99$ & $<1$ & $<, 1$ & 19 & 3 & 460 & 11 & 2 \\
\hline & $06-22-99$ & $<1$ & $<.1$ & 20 & 3 & 380 & 12 & 2 \\
\hline \multirow[t]{2}{*}{12324200} & $02-22-99$ & $<1$ & $<.1$ & 16 & 5 & 220 & $<10$ & 2 \\
\hline & $02-22-99$ & $<1$ & $<1$ & 18 & 4 & 260 & $<10$ & 2 \\
\hline \multirow[t]{2}{*}{12331800} & $08-13-99$ & $<1$ & $<.1$ & 19 & 5 & 370 & $<10$ & 3 \\
\hline & $08-13-99$ & $<1$ & $<.1$ & 18 & 5 & 360 & $<10$ & 3 \\
\hline \multirow[t]{2}{*}{12334550} & $05-12-99$ & $<1$ & $<1$ & 13 & 3 & 310 & 16 & 2 \\
\hline & $05-12-99$ & $<1$ & $<.1$ & 12 & 3 & 320 & 18 & 2 \\
\hline \multirow[t]{2}{*}{12340500} & $05-26-99$ & $<1$ & $<.1$ & 22 & 2 & 2,200 & 49 & 5 \\
\hline & $05-26-99$ & $<1$ & $<.1$ & 22 & 2 & 2,400 & 45 & 6 \\
\hline
\end{tabular}

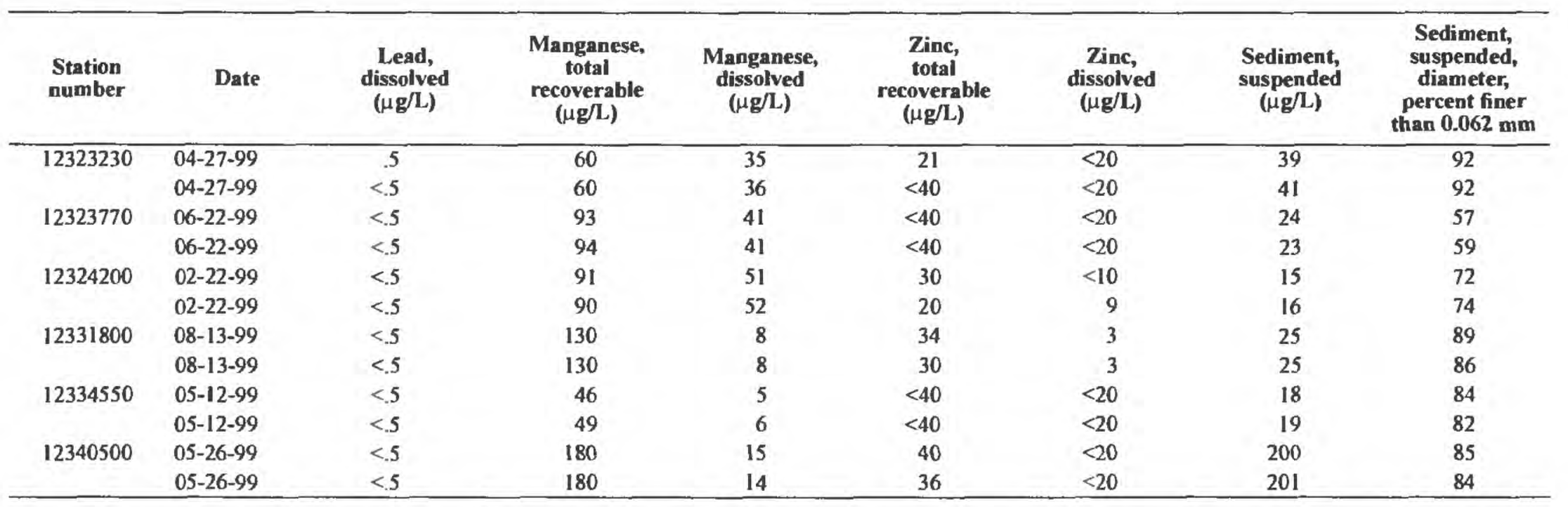


Table 9. Precision of chemical and suspended-sediment analyses of field replicates for water samples, upper Clark Fork basin, Montana

[Abbreviations: $\mu \mathrm{g} / \mathrm{L}$, micrograms per liter; $\mathrm{mg} / \mathrm{L}$. milligrams per liter; $\mathrm{mm}$, millimeter]

\begin{tabular}{llcc}
\hline \multicolumn{1}{c}{ Constituent and reporting unit } & $\begin{array}{c}\text { Number of } \\
\text { replicate pairs }\end{array}$ & $\begin{array}{c}\text { Standard } \\
\text { deviation, } \\
\text { in units }(+/ \text {-) }\end{array}$ & $\begin{array}{c}\text { Relative } \\
\text { standard } \\
\text { deviation, } \\
\text { in percent (+l-) }\end{array}$ \\
\hline Calcium, dissolved, $\mathrm{mg} / \mathrm{L}$ & 6 & 0.50 & 1.3 \\
Magnesium, dissolved, $\mathrm{mg} / \mathrm{L}$ & 6 & .29 & 3.1 \\
Arsenic, total recoverable, $\mu \mathrm{g} / \mathrm{L}$ & 6 & .41 & 5.7 \\
Arsenic, dissolved, $\mu \mathrm{g} / \mathrm{L}$ & 6 & .71 & 12 \\
Cadmium, total recoverable, $\mu \mathrm{g} / \mathrm{L}$ & 6 & .0 & .0 \\
Cadmium, dissolved, $\mu \mathrm{g} / \mathrm{L}$ & 6 & .0 & .0 \\
Copper, total recoverable, $\mu \mathrm{g} / \mathrm{L}$ & 6 & .76 & 4.7 \\
Copper, dissolved, $\mu \mathrm{g} / \mathrm{L}$ & 6 & .29 & 8.5 \\
Iron, total recoverable, $\mu \mathrm{g} / \mathrm{L}$ & 6 & 63 & 8.5 \\
Iron, dissolved, $\mu \mathrm{g} / \mathrm{L}$ & 6 & 3.2 & 6.3 \\
Lead, total recoverable, $\mu \mathrm{g} / \mathrm{L}$ & 6 & .29 & 8.9 \\
Lead, dissolved, $\mu \mathrm{g} / \mathrm{L}$ & 6 & .0 & .0 \\
Manganese, total recoverable, $\mu \mathrm{g} / \mathrm{L}$ & 6 & .96 & 1.0 \\
Manganese, dissolved, $\mu \mathrm{g} / \mathrm{L}$ & 6 & .58 & 2.2 \\
Zinc, total recoverable, $\mu \mathrm{g} / \mathrm{L}$ & 6 & 3.3 & 10 \\
Zinc, dissolved, $\mu \mathrm{g} / \mathrm{L}$ & 6 & .29 & 1.8 \\
Sediment, suspended, $\mathrm{mg} / \mathrm{L}$ & 6 & .82 & 1.5 \\
Sediment, suspended, percent finer than $0.062 \mathrm{~mm}$ & 6 & 1.4 & 1.7 \\
\hline
\end{tabular}


Table 10. Precision of chemical analyses of laboratory replicates for water samples. upper Clark Fork basin. Montana [Abbreviations. $\mu \mathrm{g} / \mathrm{L}$. micrograms per liter, $\mathrm{mg} / \mathrm{L}$. milligrams per liter]

\begin{tabular}{lcccc}
\hline \multicolumn{1}{c}{ Constituent and reporting unit } & $\begin{array}{c}\text { Number of } \\
\text { replicate pairs }\end{array}$ & $\begin{array}{c}\text { Standard } \\
\text { deviation, } \\
\text { in units }(+/ \text {-) }\end{array}$ & $\begin{array}{c}\text { Relative } \\
\text { standard } \\
\text { deviation, } \\
\text { in percent (+/) }\end{array}$ & $\begin{array}{c}\text { Within limits of } \\
\text { data-quality } \\
\text { objective }\end{array}$ \\
\hline Calcium, dissolved, $\mathrm{mg} / \mathrm{L}$ & 10 & 0.16 & 0.5 & Yes \\
Magnesium. dissolved, $\mathrm{mg} / \mathrm{L}$ & 10 & .12 & 1.6 & Yes \\
Arsenic, total recoverable, $\mu \mathrm{g} / \mathrm{L}$ & 10 & .44 & 5.6 & Yes \\
Arsenic, dissolved, $\mu \mathrm{g} / \mathrm{L}$ & 10 & .25 & 4.1 & Yes \\
Cadmium, total recoverable, $\mu \mathrm{g} / \mathrm{L}$ & 10 & .02 & 16 & Yes \\
Cadmium, dissolved, $\mu \mathrm{g} / \mathrm{L}$ & 10 & .00 & $<.1$ & Yes \\
Copper, total recoverable, $\mu \mathrm{g} / \mathrm{L}$ & 10 & .43 & 3.8 & Yes \\
Copper, dissolved, $\mu \mathrm{g} / \mathrm{L}$ & 10 & .34 & 9.9 & Yes \\
Iron, total recoverable, $\mu \mathrm{g} / \mathrm{L}$ & 10 & 5.6 & .9 & Yes \\
Iron, dissolved, $\mu \mathrm{g} / \mathrm{L}$ & 10 & .73 & .8 & Yes \\
Lead, total recoverable, $\mu \mathrm{g} / \mathrm{L}$ & 10 & .23 & 10 & Yes \\
Lead, dissolved, $\mu \mathrm{g} / \mathrm{L}$ & 10 & .00 & $<.1$ & Yes \\
Manganese, total recoverable, $\mu \mathrm{g} / \mathrm{L}$ & 10 & .64 & .8 & Yes \\
Manganese, dissolved, $\mu \mathrm{g} / \mathrm{L}$ & 10 & .31 & .9 & Yes \\
Zinc, total recoverable, $\mu \mathrm{g} / \mathrm{L}$ & 10 & 3.0 & 18 & Yes \\
Zinc, dissolved, $\mu \mathrm{g} / \mathrm{L}$ & 10 & .89 & 36 & 'No \\
\hline
\end{tabular}

${ }^{1}$ Statistics calculated using laboratory reporting level for values less than the detection capability of the instrument. 
Table 11. Recovery efficiency for trace-element analyses of laboratory-spiked deionized-water blanks [Abbreviation $\mu \mathrm{g} / \mathrm{L}$, micrograms per liter]

\begin{tabular}{|c|c|c|c|c|}
\hline Constituent and reporting unit & $\begin{array}{c}\text { Number of } \\
\text { samples }\end{array}$ & $\begin{array}{l}\text { 95-percent } \\
\text { confidence } \\
\text { interval for spike } \\
\text { recovery, } \\
\text { in percent }\end{array}$ & $\begin{array}{l}\text { Mean spike } \\
\text { recovery, } \\
\text { in percent }\end{array}$ & $\begin{array}{l}\text { Within limits of } \\
\text { data-quality } \\
\text { objective }\end{array}$ \\
\hline Arsenic, total recoverable, $\mu \mathrm{g} / \mathrm{L}$ & 5 & $97.8-108$ & 102.9 & Yes \\
\hline Arsenic, dissolved, $\mu \mathrm{g} / \mathrm{L}$ & 5 & $95.4-122$ & 108.9 & Yes \\
\hline Cadmium. total recoverable, $\mu \mathrm{g} / \mathrm{L}$ & 5 & $99.9-107$ & 103.4 & Yes \\
\hline Cadmium, dissolved, $\mu \mathrm{g} / \mathrm{L}$ & 5 & $92.6-109$ & 100.9 & Yes \\
\hline Copper, total recoverable, $\mu \mathrm{g} / \mathrm{L}$ & 5 & $102-108$ & 105.0 & Yes \\
\hline Copper, dissolved. $\mu \mathrm{g} / \mathrm{L}$ & 5 & $99.5-116$ & 107.5 & Yes \\
\hline Iron, total recoverable, $\mu \mathrm{g} / \mathrm{L}$ & 5 & $94.3-101$ & 97.7 & Yes \\
\hline Iron, dissolved, $\mu \mathrm{g} / \mathrm{L}$ & 5 & $90.0-103$ & 96.5 & Yes \\
\hline Lead, total recoverable, $\mu \mathrm{g} / \mathrm{L}$ & 5 & $85.2-107$ & 96.2 & Yes \\
\hline Lead, dissolved, $\mu \mathrm{g} / \mathrm{L}$ & 5 & $87.9-107$ & 97.4 & Yes \\
\hline Manganese, total recoverable, $\mu \mathrm{g} / \mathrm{L}$ & 5 & $91.8-100$ & 96.0 & Yes \\
\hline Manganese, dissolved, $\mu \mathrm{g} / \mathrm{L}$ & 5 & $86.3-106$ & 95.9 & Yes \\
\hline Zinc, total recoverable, $\mu \mathrm{g} / \mathrm{L}$ & 5 & $88.9-97.7$ & 93.3 & Yes \\
\hline Zinc, dissolved, $\mu \mathrm{g} / \mathrm{L}$ & 5 & $92.9-101$ & 96.9 & Yes \\
\hline
\end{tabular}


Table 12. Recovery efficiency for trace-element analyses of laboratory-spiked stream samples. upper Clark Fork basin, Montana [Abbreviation: $\mu \mathrm{g} / \mathrm{L}$, micrograms per liter]

\begin{tabular}{|c|c|c|c|c|}
\hline Constituent and reporting unit & Number of samples & $\begin{array}{l}\text { 95-percent } \\
\text { confidence interval for } \\
\text { spike recovery, } \\
\text { in percent }\end{array}$ & $\begin{array}{l}\text { Mean spike } \\
\text { recovery, } \\
\text { in percent }\end{array}$ & $\begin{array}{l}\text { Within limits of } \\
\text { data-quality } \\
\text { objective }\end{array}$ \\
\hline Arsenic, total recoverable, $\mu \mathrm{g} / \mathrm{L}$ & 5 & $96.4-111$ & 103.6 & Yes \\
\hline Arsenic, dissolved, $\mu \mathrm{g} / \mathrm{L}$ & 5 & $97.5-113$ & 105.2 & Yes \\
\hline Cadmium, total recoverable, $\mu \mathrm{g} / \mathrm{L}$ & 5 & $100-106$ & 103.0 & Yes \\
\hline Cadmium, dissolved, $\mu \mathrm{g} / \mathrm{L}$ & 5 & $92.4-113$ & 102.6 & Yes \\
\hline Copper, total recoverable, $\mu \mathrm{g} / \mathrm{L}$ & 5 & $97.5-104$ & 100.5 & Yes \\
\hline Copper, dissolved, $\mu \mathrm{g} / \mathrm{L}$ & 5 & $89.1-116$ & 102.7 & Yes \\
\hline Iron, total recoverable, $\mu \mathrm{g} / \mathrm{L}$ & 5 & $93.5-101$ & 97.2 & Yes \\
\hline Iron, dissolved, $\mu \mathrm{g} / \mathrm{L}$ & 5 & $90.6-109$ & 99.9 & Yes \\
\hline Lead, total recoverable, $\mu \mathrm{g} / \mathrm{L}$ & 5 & $93.7-108$ & 101.1 & Yes \\
\hline Lead, dissolved, $\mu \mathrm{g} / \mathrm{L}$ & 5 & $94.0-106$ & 100.2 & Yes \\
\hline Manganese, total recoverable, $\mu \mathrm{g} / \mathrm{L}$ & 5 & $92.8-107$ & 99.8 & Yes \\
\hline Manganese, dissolved, $\mu \mathrm{g} / \mathrm{L}$ & 5 & $89.3-108$ & 98.8 & Yes \\
\hline Zinc, total recoverable, $\mu \mathrm{g} / \mathrm{L}$ & 5 & $91.1-101$ & 96.2 & Yes \\
\hline Zinc, dissolved, $\mu \mathrm{g} / \mathrm{L}$ & 5 & $95.7-102$ & 98.6 & Yes \\
\hline
\end{tabular}


Table 13. Chemical analyses of field blanks for water samples

[Abbreviations: ${ }^{\circ} \mathrm{C}$, degrees Celsius; $\mu \mathrm{g} / \mathrm{L}$, micrograms per liter, E, estimated; $\mu \mathrm{S} / \mathrm{cm}$, microsiemens per centimeter at $25^{\circ} \mathrm{C}$; $\mathrm{mg} / \mathrm{L}, \mathrm{milligrams}$ per liter. Symbol: <, less than laboratory reporting level]

\begin{tabular}{|c|c|c|c|c|c|c|c|c|c|c|}
\hline Date & Time & $\begin{array}{c}\text { Specific } \\
\text { conduct- } \\
\text { ance, } \\
\text { onsife } \\
(\mu \mathrm{S} / \mathrm{cm})\end{array}$ & $\begin{array}{c}\text { pH, } \\
\text { onsite } \\
\text { (standard } \\
\text { units) }\end{array}$ & $\begin{array}{l}\text { Calcium, } \\
\text { dissolved } \\
(\mathrm{mg} / \mathrm{L})\end{array}$ & $\begin{array}{c}\text { Magne- } \\
\text { sium, } \\
\text { dissolved } \\
(\mathrm{mg} / \mathrm{L})\end{array}$ & $\begin{array}{c}\text { Arsenic, } \\
\text { total } \\
\text { recov- } \\
\text { erable } \\
(\mu \mathrm{g} / \mathrm{L})\end{array}$ & $\begin{array}{c}\text { Arsenic, } \\
\text { dissolved } \\
(\mu \mathrm{g} / \mathrm{L})\end{array}$ & $\begin{array}{c}\text { Cadmium, } \\
\text { total } \\
\text { recoverable } \\
(\mu \mathrm{g} / \mathrm{L})\end{array}$ & $\begin{array}{c}\text { Cadmium, } \\
\text { dissolved } \\
(\mu \mathrm{g} / \mathrm{L})\end{array}$ & $\begin{array}{c}\text { Copper, } \\
\text { total } \\
\text { recoverable } \\
(\mu \mathrm{g} / \mathrm{L})\end{array}$ \\
\hline \multicolumn{11}{|c|}{ NOV 1998} \\
\hline $17 \ldots$ & 1800 & 3 & 5.5 & E001 & E0.002 & $<1$ & $<1$ & $<I$ & $<0.1$ & $<1$ \\
\hline \multicolumn{11}{|c|}{ FEB 1999} \\
\hline $22 \ldots$ & 1800 & 2 & 5.6 & E.02 & E 003 & $<1$ & $<1$ & $<1$ & $<1$ & $<1$ \\
\hline \multicolumn{11}{|l|}{ APR } \\
\hline $28 \ldots$ & 1600 & 3 & 54 & E.01 & E.002 & $<1$ & $<1$ & $<1$ & $<1$ & $<1$ \\
\hline \multicolumn{11}{|l|}{ MAY } \\
\hline 11. & 1700 & 2 & 5.5 & $<.02$ & $<.004$ & $<1$ & $<1$ & $<1$ & $<.1$ & $<1$ \\
\hline $27 \ldots$ & 0845 & 3 & 5.6 & E.02 & .006 & $<1$ & $<1$ & $<1$ & $<.1$ & $<1$ \\
\hline \multicolumn{11}{|l|}{ JUN } \\
\hline $10 \ldots$ & 0900 & 2 & 5.6 & E.01 & $<.004$ & $<1$ & $<1$ & $<1$ & $<.1$ & $<1$ \\
\hline $23 \ldots$ & 0700 & 2 & 5.7 & E.01 & E.002 & $<1$ & $<1$ & $<1$ & $<.1$ & $<1$ \\
\hline \multicolumn{11}{|l|}{ AUG } \\
\hline 13. & 1245 & 2 & 62 & .01 & E 002 & $<1$ & $<1$ & $<1$ & $<1$ & $<1$ \\
\hline
\end{tabular}

\begin{tabular}{|c|c|c|c|c|c|c|c|c|c|}
\hline Date & $\begin{array}{c}\text { Copper, } \\
\text { dissolved } \\
(\mu \mathrm{g} / \mathrm{L})\end{array}$ & $\begin{array}{c}\text { Iron, } \\
\text { total } \\
\text { recoverable } \\
(\mu \mathrm{g} / \mathrm{L})\end{array}$ & $\begin{array}{c}\text { Iron, } \\
\text { dissolved } \\
(\mu \mathrm{g} / \mathrm{L})\end{array}$ & $\begin{array}{c}\text { Lead, } \\
\text { total } \\
\text { recoverable } \\
(\mu \mathrm{g} / \mathrm{L})\end{array}$ & $\begin{array}{c}\text { Lead, } \\
\text { dissolved } \\
(\mu \mathrm{g} / \mathrm{L})\end{array}$ & $\begin{array}{l}\text { Manga- } \\
\text { nese, } \\
\text { total } \\
\text { recoverable } \\
(\mu \mathrm{g} / \mathrm{L})\end{array}$ & $\begin{array}{c}\text { Manganese, } \\
\text { dissolved } \\
(\mu \mathrm{g} / \mathrm{L})\end{array}$ & $\begin{array}{c}\text { Zinc, } \\
\text { total } \\
\text { recoverable } \\
(\mu \mathrm{g} / \mathrm{L})\end{array}$ & $\begin{array}{c}\text { Zinc, } \\
\text { dissolved } \\
(\mu \mathrm{g} / \mathrm{L})\end{array}$ \\
\hline \multicolumn{10}{|l|}{ NOV 1998} \\
\hline 17 & $<1$ & $<10$ & $<10$ & $<1$ & $<0.5$ & $<10$ & $<4$ & $<10$ & $<20$ \\
\hline \multicolumn{10}{|l|}{ FEB 1999} \\
\hline $22 \ldots$ & $<1$ & $<10$ & $<10$ & $<1$ & $<.5$ & $<10$ & $<3$ & $<10$ & $<20$ \\
\hline \multicolumn{10}{|l|}{ APR } \\
\hline $28 \ldots$ & $<1$ & $<14$ & $<10$ & $<1$ & $<.5$ & $<3$ & $<3$ & $<40$ & $<20$ \\
\hline \multicolumn{10}{|l|}{ MAY } \\
\hline $11 \ldots$ & $<1$ & $<14$ & $<10$ & $<1$ & $<, 5$ & $<3$ & $<3$ & $<40$ & $<20$ \\
\hline $27 \ldots$ & $<1$ & $<14$ & $<10$ & $<1$ & $<.5$ & $<3$ & $<3$ & $<40$ & $<20$ \\
\hline \multicolumn{10}{|l|}{ JUN } \\
\hline $10 \ldots$ & $<1$ & $<14$ & $<10$ & $<1$ & $<.5$ & $<3$ & $<3$ & $<40$ & $<20$ \\
\hline $23 \ldots$ & $<1$ & $<14$ & $<10$ & $<1$ & $<.5$ & $<3$ & $<3$ & $<40$ & $<20$ \\
\hline \multicolumn{10}{|l|}{ AUG } \\
\hline $13 \ldots$ & $<1$ & 36 & $<10$ & $<1$ & $<.5$ & $<3$ & $<1$ & $<40$ & $<1$ \\
\hline
\end{tabular}


Table 14. Trace-element analyses of fine-grained bed sediment. upper Clark Fork basin, Montana, August 1999

[Fine-grained sediment is material less than 0.064 millimeter in diameter. Reported concentrations are the mean of all analyses for replicate aliquots from each composite sample. Abbreviation: $\mu \mathrm{g} / \mathrm{g}$, micrograms per gram of dry sample weight. Symbol: <, less than]

\begin{tabular}{|c|c|c|c|c|c|c|c|c|c|c|c|}
\hline \multirow{2}{*}{$\begin{array}{l}\text { Station number } \\
\text { (fig. 1) }\end{array}$} & \multirow{2}{*}{ Station name } & \multirow{2}{*}{$\begin{array}{c}\text { Number of } \\
\text { com- } \\
\text { posite } \\
\text { samples }\end{array}$} & \multicolumn{9}{|c|}{ Concentration, in $\mu \mathrm{g} / \mathrm{g}$} \\
\hline & & & $\begin{array}{l}\text { Cad- } \\
\text { mium }\end{array}$ & $\begin{array}{l}\text { Chro- } \\
\text { mium }\end{array}$ & $\begin{array}{l}\text { Cop- } \\
\text { per }\end{array}$ & Iron & Lead & $\begin{array}{c}\text { Manga- } \\
\text { nese }\end{array}$ & Nickel & Silver & Zinc \\
\hline 12323600 & Silver Bow Creek at Opportunity & 3 & 26.5 & 24.8 & 5,970 & 34,600 & 852 & 2,200 & 14.2 & 18.9 & 6,600 \\
\hline 12323750 & Silver Bow Creek at Warm Springs & 3 & 4.5 & 23.6 & 223 & 20,000 & 62 & 3,100 & 15.7 & 1.6 & 686 \\
\hline 12323770 & Warm Springs Creek at Warm Springs & 3 & 1.3 & 27.5 & 779 & 20,600 & 85 & 6,030 & 19.6 & 3.1 & 396 \\
\hline 12323800 & Clark Fork near Galen & 3 & 4.0 & 27.0 & 991 & 26,800 & 136 & 6,330 & 19.0 & 3.6 & 1,120 \\
\hline 461415112450801 & $\begin{array}{l}\text { Clark Fork below Lost Creek, } \\
\text { near Galen }\end{array}$ & 3 & 6.5 & 27.5 & 1,440 & 31,700 & 174 & 5,000 & 18.7 & 5.6 & 1,490 \\
\hline 461559112443301 & Clark Fork near Racetrack & 3 & 5.0 & 24.9 & 1,390 & 28,600 & 186 & 4,030 & 17.0 & 4.8 & 1,430 \\
\hline 461903112440701 & $\begin{array}{l}\text { Clark Fork at Dempsey Creek } \\
\text { diversion, near Racetrack }\end{array}$ & 3 & 4.3 & 26.4 & 766 & 28,300 & 117 & 1,810 & 15.1 & 2.7 & 907 \\
\hline 12324200 & Clark Fork at Deer Lodge & 3 & 4.4 & 32.5 & 1,070 & 30,200 & 150 & 4,910 & 20.1 & 4.5 & 1,320 \\
\hline 12324680 & Clark Fork at Goldcreek & 3 & 3.5 & 29.4 & 780 & 25,300 & 119 & 1,660 & 15.9 & 3.2 & 1,080 \\
\hline 12331500 & Flint Creek near Drummond & 3 & $<.2$ & 20.4 & 63 & 23,400 & 162 & 4,570 & 12.7 & 5.8 & 650 \\
\hline 12331800 & Clark Fork near Drummond & 3 & 2.6 & 27.0 & 491 & 23,200 & 94 & 1,740 & 15.0 & 2.3 & 947 \\
\hline 12334510 & Rock Creek near Clinton & 3 & $<.2$ & 20.3 & 16 & 19,300 & 15 & 478 & 14.8 & $<.4$ & 47 \\
\hline 12334550 & $\begin{array}{l}\text { Clark Fork at Turah Bridge, } \\
\text { near Bonner }\end{array}$ & 3 & 3.9 & 27.7 & 479 & 24,400 & 102 & 1,080 & 19.1 & 1.0 & 1,080 \\
\hline 12340000 & Blackfoot River near Bonner & 3 & $<.2$ & 21.6 & 27 & 20,100 & 19 & 544 & 14.3 & $<.4$ & 67 \\
\hline 12340500 & Clark Fork above Missoula & 3 & 1.5 & 20.8 & 219 & 20,400 & 47 & 1,370 & 15.8 & .8 & 547 \\
\hline 12353000 & Clark Fork below Missoula & 3 & 1.2 & 17.2 & 141 & 17,300 & 41 & 1,080 & 12.5 & .4 & 380 \\
\hline
\end{tabular}

'Samples collected about 30 miles downstream from water-quality station 10 conform 10 previous sampling location. 
Table 15. Trace-element analyses of bulk bed sediment, upper Clark Fork basin, Montana, August 1999

[Bulk bed sediment collected in this study generally is material smaller than about 10 millimeters in diameter. Reported concentrations are the mean of all analyses for replicate aliquots of each composite sample. Abbreviation: $\mu \mathrm{g} / \mathrm{g}$, micrograms per gram of dry sample weight. Symbol: <, less than]

\begin{tabular}{|c|c|c|c|c|c|c|c|c|c|c|c|}
\hline \multirow{2}{*}{$\begin{array}{l}\text { Station number } \\
\text { (fig. 1) }\end{array}$} & \multirow{2}{*}{ Station name } & \multirow{2}{*}{$\begin{array}{l}\text { Number } \\
\text { of com- } \\
\text { posite } \\
\text { samples }\end{array}$} & \multicolumn{9}{|c|}{ Concentration, in $\mu \mathrm{g} / \mathrm{g}$} \\
\hline & & & $\begin{array}{l}\text { Cad- } \\
\text { mium }\end{array}$ & $\begin{array}{l}\text { Chro- } \\
\text { mium }\end{array}$ & $\begin{array}{l}\text { Cop- } \\
\text { per }\end{array}$ & Iron & Lead & $\begin{array}{l}\text { Manga- } \\
\text { nese }\end{array}$ & Nickel & Silver & Zinc \\
\hline 12323600 & Silver Bow Creek at Opportunity & 2 & 4.2 & 11.8 & 902 & 18,300 & 198 & 748 & 6.5 & 3.2 & 1,730 \\
\hline 12323750 & Silver Bow Creek at Warm Springs & 2 & $<9$ & 9.1 & 30 & 9,600 & 10 & 1,000 & 5.4 & $<.8$ & 131 \\
\hline 12323800 & Clark Fork near Galen & 2 & $<.9$ & 15.0 & 318 & 20.600 & 48 & 1,900 & 6.9 & 9 & 477 \\
\hline 461415112450801 & Clark Fork below Lost Creek, near Galen & 2 & $<.9$ & 9.3 & 238 & 12,600 & 41 & 1,290 & 4.2 & .8 & 365 \\
\hline 461559112443301 & Clark Fork near Racetrack & 2 & $<.9$ & 14.7 & 440 & 19,700 & 76 & 1,280 & 6.5 & 1.4 & 626 \\
\hline 12331500 & Flint Creek near Drummond & 2 & $<2$ & 6.5 & 18 & 10,100 & 63 & 1,800 & 4.5 & 4.1 & 220 \\
\hline 12331800 & Clark Fork near Drummond & 2 & 1.0 & 16.7 & 223 & 15,200 & 50 & 949 & 9.8 & .7 & 603 \\
\hline 12334510 & Rock Creek near Clinton & 2 & $<.2$ & 64 & 3 & 5,290 & $<3$ & 72 & 3.6 & $<.4$ & 10 \\
\hline 12334550 & Clark Fork at Turah Bridge, near Bonner & 2 & 4 & 10.1 & 84 & 9,270 & 22 & 299 & 6.7 & $<.4$ & 271 \\
\hline 1234000 & Blackfoot River near Bonner & 2 & $<.2$ & 8.7 & 12 & 11,500 & 6 & 207 & 7.5 & $<4$ & 34 \\
\hline 12340500 & Clark Fork above Missoula & 2 & 4 & 10.2 & 72 & 13,800 & 18 & 810 & 9.2 & $<.4$ & 263 \\
\hline
\end{tabular}

'Samples collected about 30 miles downstream from water-quality station to conform to previous sampling location 
Table 16. Recovery efficiency for trace-element analyses of standard reference materials for bed sediment

[Abbreviations: $\mu \mathrm{g} / \mathrm{g}$. micrograms per gram of dry sample weight; SRM, standard reference material. Symbol: --, recovery could not be determined because all analyses were less than the analytical detection limit for lead and silver]

\begin{tabular}{|c|c|c|c|c|c|}
\hline Constituent & $\begin{array}{l}\text { Number of } \\
\text { measurements }\end{array}$ & Dilution ratio & $\begin{array}{l}\text { Certified concentration } \\
\qquad(\mu \mathrm{g} / \mathrm{g})\end{array}$ & $\begin{array}{c}\text { Mean SRM recovery } \\
\text { (percent) }\end{array}$ & $\begin{array}{l}\text { 95-percent confidence } \\
\text { interval for SRM } \\
\text { recovery } \\
\text { (percent) }\end{array}$ \\
\hline \multicolumn{6}{|c|}{ SRM sample 2709} \\
\hline Cadmium & 6 & $1: 5$ & 0.4 & 133.9 & $83.5-184$ \\
\hline Chromium & 6 & $1: 5$ & 130 & 65.6 & $61.2-70.0$ \\
\hline Copper & 6 & 1:5 & 35 & 90.0 & $85.8-94.2$ \\
\hline Iron & 6 & $1: 5$ & 35,000 & 88.0 & $85.7-90.3$ \\
\hline Lead & 6 & $1: 5$ & 19 & 87.0 & $82.2-91.8$ \\
\hline Manganese & 6 & $1: 5$ & 538 & 94.0 & $91.2-96.8$ \\
\hline Nickel & 6 & 1:5 & 88 & 87.9 & $85.9-89.9$ \\
\hline Silver & 6 & $1: 5$ & .4 & - & - \\
\hline Zinc & 6 & $1: 5$ & 106 & 95.0 & $89.4-100$ \\
\hline \multicolumn{6}{|c|}{ SRM sample 2711} \\
\hline Cadmium & 6 & $1: 10$ & 41.7 & 98.5 & $97.1-99.9$ \\
\hline Chromium & 6 & $1: 10$ & 47.0 & 57.9 & $54.9-60.9$ \\
\hline Copper & 6 & $1: 10$ & 114 & 95.9 & $93.2-98.6$ \\
\hline Iron & 6 & $1: 10$ & 28,900 & 83.0 & $81.1-84.9$ \\
\hline Lead & 6 & 1:10 & 1,160 & 95.6 & $94.1-97.1$ \\
\hline Manganese & 6 & 1:10 & 638 & 85.1 & $83.9-86.3$ \\
\hline Nickel & 6 & $1: 10$ & 20.6 & 91.8 & $85.9-97.7$ \\
\hline Silver & 6 & $1: 10$ & 4.6 & 76.9 & $73.8-80.0$ \\
\hline Zinc & 6 & $1: 10$ & 350 & 100.9 & $92.3-109.5$ \\
\hline
\end{tabular}




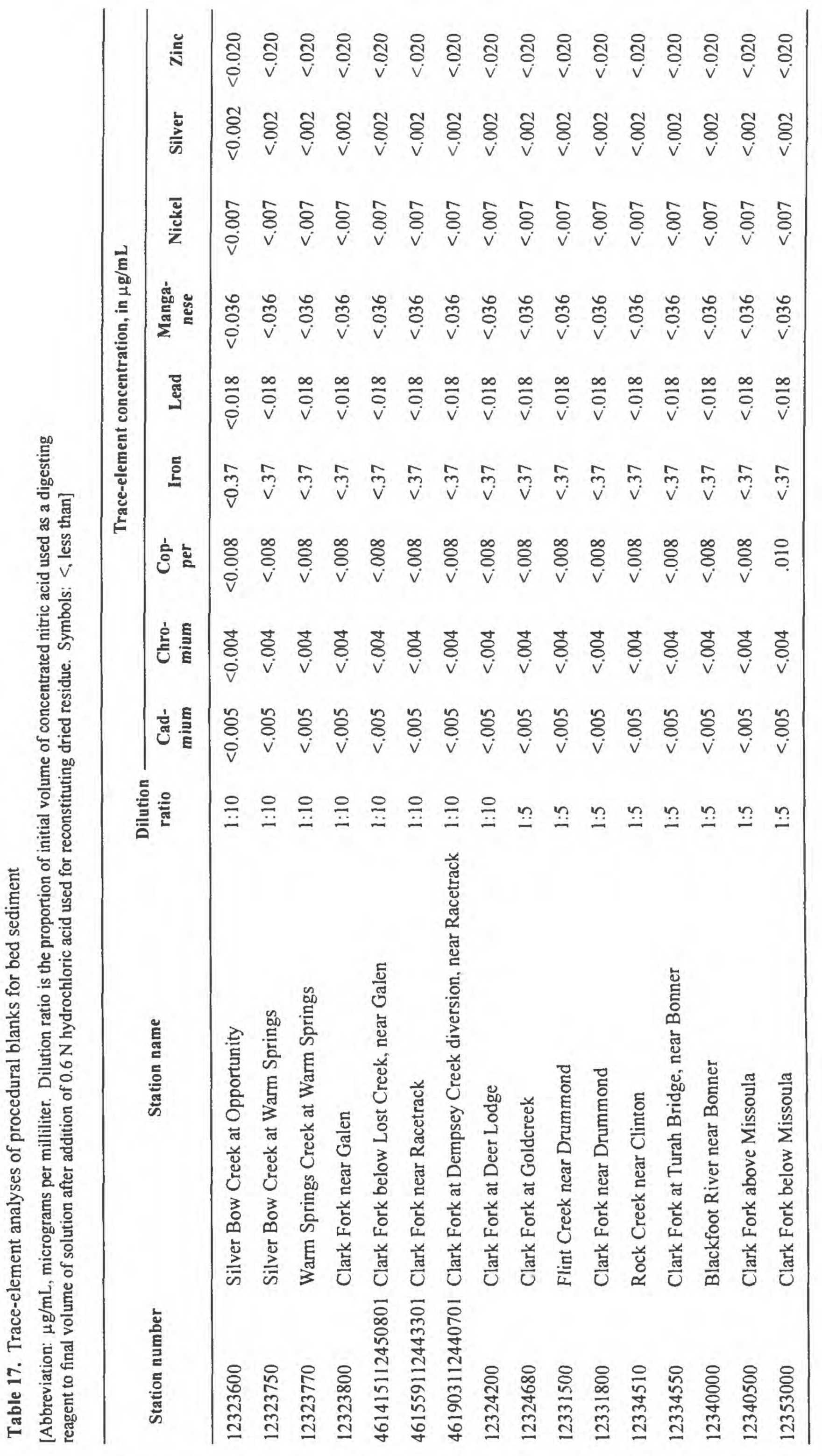

56 Water-quality, bed-sediment, and biological data (October 1998 through September 1999) and statistical summaries of data for streams in the Upper Clark Fork Basin, Montana 
Table 18. Trace-element analyses of biota. upper Clark Fork basin, Montana, August 1999

[Analyses are of whole-body tissue of aquatic insects. Composite samples made by combining similar-sized insects of the same species into a sample of sufficient mass for analysis. Concentrations for biota samples composed of two or more composite samples are the means of all analyses. Abbreviations: $\mu \mathrm{g} / \mathrm{g}$, micrograms per gram of dry sample weight. Symbol: $<$, less than minimum reporting level]

\begin{tabular}{|c|c|c|c|c|c|c|c|c|c|}
\hline \multirow[b]{2}{*}{ Taxon } & \multirow{2}{*}{$\begin{array}{l}\text { Number of } \\
\text { com- } \\
\text { posite } \\
\text { samples }\end{array}$} & \multicolumn{8}{|c|}{ Concentration, in $\mu \mathrm{g} / \mathrm{g}$} \\
\hline & & $\begin{array}{l}\text { Cad- } \\
\text { mium }\end{array}$ & $\begin{array}{l}\text { Chro- } \\
\text { mium }\end{array}$ & $\begin{array}{l}\text { Cop- } \\
\text { per }\end{array}$ & Iron & Lead & $\begin{array}{l}\text { Manga- } \\
\text { nese }\end{array}$ & Nickel & Zinc \\
\hline \multicolumn{10}{|c|}{12323600 Silver Bow Creek at Opportunity } \\
\hline Brachycentrus spp. & 1 & 5.8 & 0.7 & 846 & 730 & 17.9 & 503 & 1.9 & 629 \\
\hline \multicolumn{10}{|c|}{12323750 Silver Bow Creek at Warm Springs } \\
\hline Hydropsyche cockerelli & 1 & $<4$ & $<4$ & 22.4 & 453 & $<2.1$ & 657 & $<1.7$ & 115 \\
\hline Hydropsyche occidentalis & 5 & .2 & 1.0 & 28.9 & 761 & 3.7 & 1,690 & 9 & 174 \\
\hline \multicolumn{10}{|c|}{12323770 Warm Springs Creek at Warm Springs } \\
\hline Arctopsyche grandis & 1 & 1.9 & 2.9 & 102 & 872 & $<8.7$ & 1,800 & $<7.0$ & 180 \\
\hline Hydropsyche occidentalis & 2 & .7 & 3.2 & 182 & 2,010 & 7.4 & 2,440 & 3.2 & 169 \\
\hline \multicolumn{10}{|c|}{12323800 Clark Fork near Galen } \\
\hline Hydropsyche cockerelli & I & 1.4 & 1.5 & 48.7 & 816 & 6.3 & 1,370 & $<4.3$ & 155 \\
\hline Hydropsyche occidentalis & 4 & .7 & 1.4 & 67.5 & 1,100 & 6.1 & 1,950 & 1.3 & 178 \\
\hline \multicolumn{10}{|c|}{ 461415112450801 Clark Fork below Lost Creek, near Galen } \\
\hline Hydropsyche cockerelli & 1 & 2.3 & 1.0 & 48.8 & 706 & $<3.0$ & 1,230 & $<2.4$ & 151 \\
\hline Hydropsyche occidentalis & 4 & 1.1 & 1.7 & 93.6 & 1,380 & 10.0 & 3,240 & 1.5 & 254 \\
\hline Hydropsyche spp. & 1 & 1.3 & 1.0 & 45.1 & 533 & 4.1 & 1,230 & $<1.6$ & 143 \\
\hline \multicolumn{10}{|c|}{461559112443301 Clark Fork near Racetrack } \\
\hline Hydropsyche cockerelli & 1 & $<2.2$ & 2.2 & 50.0 & 846 & $<10.9$ & 1,690 & $<8.7$ & 155 \\
\hline Hydropsyche occidentalis & 2 & .8 & 1.3 & 64.1 & 1,190 & 6.7 & 3,220 & 1.3 & 194 \\
\hline \multicolumn{10}{|c|}{ 461903112440701 Clark Fork at Dempsey Creek diversion, near Racetrack } \\
\hline Hydropsyche occidentalis & 5 & 1.0 & 1.8 & 88.3 & 1,530 & 11.8 & 3,610 & 1.7 & 277 \\
\hline \multicolumn{10}{|c|}{12324200 Clark Fork at Deer Lodge } \\
\hline Hydropsyche cockerelli & 1 & 1.8 & 1.6 & 57.4 & 977 & $<7.6$ & 772 & 2.2 & 134 \\
\hline Hydropsyche occidentalis & 4 & 1.2 & 1.5 & 94.3 & 1,190 & 7.7 & 1,640 & 1.6 & 216 \\
\hline \multicolumn{10}{|c|}{12324680 Clark Fork at Goldcreek } \\
\hline Arctopsyche grandis & 4 & 4.1 & 1.2 & 54.6 & 518 & 4.0 & 1,020 & 6 & 306 \\
\hline Claassenia sabulosa & 2 & .7 & .8 & 69.0 & 223 & 1.2 & 125 & .4 & 255 \\
\hline Hydropsyche cockerelli & 1 & 2.4 & 1.8 & 58.6 & 1,080 & 5.7 & 900 & 1.2 & 206 \\
\hline Hydropsyche occidentalis & 2 & 1.7 & 1.7 & 64.6 & 1,070 & 6.2 & 1,290 & 1.0 & 228 \\
\hline \multicolumn{10}{|c|}{12331500 Flint Creek near Drummond } \\
\hline Arctopsyche grandis & 2 & .5 & 1.6 & 16.1 & 1,260 & 9.1 & 1,530 & 1.6 & 202 \\
\hline Hydropsyche spp. & 1 & $<.3$ & 1.4 & 12.5 & 1,440 & 4.5 & 1,320 & 1.3 & 130 \\
\hline \multicolumn{10}{|c|}{12331800 Clark Fork near Drummond } \\
\hline Arctopsyche grandis & 2 & 1.9 & 1.2 & 36.8 & 614 & 3.6 & 1,120 & $<1.2$ & 220 \\
\hline Claassenia sabulosa & 3 & 1.6 & 1.2 & 106 & 335 & 1.9 & 343 & .5 & 368 \\
\hline Hydropsyche cockerelli & 2 & 1.0 & 1.3 & 43.8 & 927 & 2.4 & 784 & 1.3 & 142 \\
\hline Hydropsyche occidentalis & 2 & 1.2 & 2.1 & 57.6 & 1,300 & 8.8 & 1,860 & 1.7 & 249 \\
\hline
\end{tabular}


Table 18. Trace-element analyses of biota, upper Clark Fork basin. Montana, August 1999 (Continued)

\begin{tabular}{|c|c|c|c|c|c|c|c|c|c|}
\hline \multirow[b]{2}{*}{ Taxon } & \multirow{2}{*}{$\begin{array}{c}\text { Number of } \\
\text { com- } \\
\text { posite } \\
\text { samples }\end{array}$} & \multicolumn{8}{|c|}{ Concentration, in $\mu \mathrm{g} / \mathrm{g}$} \\
\hline & & $\begin{array}{l}\text { Cad- } \\
\text { mium }\end{array}$ & $\begin{array}{l}\text { Chro- } \\
\text { mium }\end{array}$ & $\begin{array}{l}\text { Cop- } \\
\text { per }\end{array}$ & Iron & Lead & $\begin{array}{c}\text { Manga- } \\
\text { nese }\end{array}$ & Nickel & Zinc \\
\hline \multicolumn{10}{|c|}{12334510 Rock Creek near Clinton } \\
\hline Arctopsyche grandis & 1 & 3 & .8 & 8.1 & 471 & $<1.4$ & 152 & 11 & 101 \\
\hline \multicolumn{10}{|c|}{12334550 Clark Fork at Tnrah Bridge, near Bonner } \\
\hline Arctopsyche grandis & 3 & 1.5 & 2.2 & 48.5 & 1,310 & 5.1 & 713 & 1.7 & 229 \\
\hline Claassenia sabulosa & 1 & 1.0 & .4 & 39.3 & 90 & 6 & 66 & $<.3$ & 196 \\
\hline Hydropsyche cockerelli & I & 1.0 & 2.5 & 62.6 & 1,510 & 5.8 & 651 & 1.6 & 220 \\
\hline Hydropsyche occidentalis & 2 & 1.1 & 2.3 & 56.6 & 1,370 & 6.1 & 744 & 1.5 & 227 \\
\hline \multicolumn{10}{|c|}{12340500 Clark Fork above Missoula } \\
\hline Arctopsyche grandis & 3 & 8 & 1.7 & 40.0 & 1,070 & 2.8 & 988 & 1.4 & 200 \\
\hline Claassenia sabulosa & 2 & .8 & .9 & 51.5 & 218 & .5 & 122 & $<.8$ & 249 \\
\hline Hydropsyche cockerelli & 1 & .8 & 2.1 & 40.4 & 1,900 & $<2.7$ & 931 & 2.3 & 156 \\
\hline Hydropsyche occidentalis & 2 & .7 & 3.2 & 51.3 & 2,300 & 5.5 & 2.370 & 2.3 & 230 \\
\hline \multicolumn{10}{|c|}{12353000 Clark Fork below Missoula $^{1}$} \\
\hline Arctopsyche grandis & 3 & 1.2 & 1.7 & 27.7 & 987 & 2.0 & 833 & 1.1 & 201 \\
\hline Claassenia sabulosa & 2 & .8 & 6 & 62.5 & 152 & .5 & 115 & $<.4$ & 251 \\
\hline Hydropsyche cockerelli & 2 & .7 & 2.2 & 38.4 & 1,560 & 2.7 & 841 & 1.5 & 167 \\
\hline Hydropsyche occidentalis & 2 & .5 & 1.5 & 25.1 & 1,000 & 1.9 & 682 & 8 & 134 \\
\hline
\end{tabular}

'Samples collected about 30 miles downstream from water-quality station to conform to previous sampling location. 
Table 19. Recovery efficiency for trace-element analyses of standard reference material for biota [Abbreviations: $\mu \mathrm{g} / \mathrm{g}$, micrograms per gram of dry sample weight; SRM, standard reference material]

\begin{tabular}{lcccc}
\hline Constituent & Number of measurements & $\begin{array}{c}\text { Certified concentration } \\
(\mu \mathrm{g} / \mathrm{g})\end{array}$ & $\begin{array}{c}\text { Mean SRM recovery } \\
\text { (percent) }\end{array}$ & $\begin{array}{c}\text { 95-percent confidence } \\
\text { interval for SRM } \\
\text { recovery } \\
\text { (percent) }\end{array}$ \\
\hline Cadmium & 12 & SRM sample TORT-2 & 102 & $101-104$ \\
Chromium & 12 & 26.7 & 156 & $155-156$ \\
Copper & 12 & .77 & 101 & $97.1-104$ \\
Iron & 12 & 106 & 100 & $94.5-106$ \\
Lead & 14 & 105 & 108 & $108-109$ \\
Manganese & 12 & .35 & 97.2 & $96.6-97.7$ \\
Nickel & 12 & 13.6 & 96.0 & $95.9-96.1$ \\
Zinc & 12 & 2.5 & 103 & $93.7-112$ \\
\hline TEigis & 180 & & 9 \\
\hline
\end{tabular}

'Eighl samples were at or below the analytical delection limis 
Table 20. Trace-element analyses of procedural blanks for biota

[Procedural blanks were not diluted prior to analysis. Abbreviation: $\mu \mathrm{g} / \mathrm{mL}$. micrograms per milliliter. Symbol: <, less than]

\begin{tabular}{|c|c|c|c|c|c|c|c|c|c|c|}
\hline \multirow{2}{*}{$\begin{array}{l}\text { Station } \\
\text { number }\end{array}$} & \multirow{2}{*}{$\begin{array}{l}\text { Station } \\
\text { name }\end{array}$} & \multirow{2}{*}{$\begin{array}{l}\text { Dilution } \\
\text { ratio }\end{array}$} & \multicolumn{8}{|c|}{ Trace-element concentration, in $\mu \mathrm{g} / \mathrm{mL}$} \\
\hline & & & $\begin{array}{l}\text { Cad- } \\
\text { mium }\end{array}$ & $\begin{array}{l}\text { Chro- } \\
\text { mium }\end{array}$ & Copper & Iron & Lead & $\begin{array}{l}\text { Manga- } \\
\text { nese }\end{array}$ & Nickel & Zinc \\
\hline 12323600 & Silver Bow Creek at Opportunity & $1: 1$ & $<0.01$ & $<0.01$ & $<0.03$ & $<0.10$ & $<0.05$ & $<0.03$ & $<0.04$ & $<0.04$ \\
\hline 12323750 & Silver Bow Creek at Warm Springs & $1: 1$ & $<.01$ & $<01$ & $<.03$ & $<.10$ & $<05$ & $<.03$ & $<.04$ & $<.04$ \\
\hline 12323770 & Warm Springs Creek at Warm Springs & $1: 1$ & $<.01$ & $<.01$ & $<.03$ & .37 & $<.05$ & $<.03$ & $<.04$ & $<.04$ \\
\hline 12323800 & Clark Fork near Galen & $1: 1$ & $<.01$ & $<.01$ & $<.03$ & $<10$ & $<.05$ & $<.03$ & $<04$ & $<.04$ \\
\hline 461415112450801 & Clark Fork below Lost Creek, near Galen & $1: 1$ & $<.01$ & $<.01$ & $<.03$ & $<.10$ & $<.05$ & $<.03$ & $<.04$ & $<.04$ \\
\hline 461559112443301 & Clark Fork near Racetrack & $1: 1$ & $<.01$ & $<.01$ & $<.03$ & $<.10$ & $<.05$ & $<.03$ & $<.04$ & $<.04$ \\
\hline 461903112440701 & $\begin{array}{l}\text { Clark Fork at Dempsey Creek diversion, } \\
\text { near Racetrack }\end{array}$ & $1: 1$ & $<.01$ & $<.01$ & $<.03$ & $<.10$ & $<05$ & $<.03$ & $<.04$ & $<.04$ \\
\hline 12324200 & Clark Fork at Deer Lodge & $1: 1$ & $<.01$ & $<.01$ & $<.03$ & $<.57$ & $<.05$ & $<.03$ & $<.04$ & $<.04$ \\
\hline 12324680 & Clark Fork at Goldcreek & $1: 1$ & $<.01$ & $<01$ & $<03$ & .45 & $<.05$ & $<.03$ & $<.04$ & $<.04$ \\
\hline 12331500 & Flint Creek near Drummond & $1: 1$ & $<.01$ & $<.01$ & $<.03$ & $<10$ & $<.05$ & $<.03$ & $<.04$ & $<.04$ \\
\hline 12331800 & Clark Fork near Drummond & $1: 1$ & $<.01$ & $<.01$ & $<.03$ & 17 & $<.05$ & $<.03$ & $<.04$ & $<.04$ \\
\hline 12334510 & Rock Creek near Clinton & 1:1 & $<.01$ & $<.01$ & $<03$ & $<.10$ & $<.05$ & $<.03$ & $<.04$ & $<04$ \\
\hline 12334550 & Clark Fork at Turah Bridge, near Bonner & $1: 1$ & $<.01$ & $<.01$ & $<.03$ & $<.10$ & $<.05$ & $<.03$ & $<.04$ & $<.04$ \\
\hline 12340500 & Clark Fork above Missoula & $1: 1$ & $<.01$ & $<.01$ & $<.03$ & $<.10$ & $<.05$ & $<.03$ & $<.04$ & $<.04$ \\
\hline 12353000 & Clark Fork below Missoula & $1: 1$ & $<.01$ & $<01$ & $<.03$ & $<10$ & $<.05$ & $<.03$ & $<.04$ & $<.04$ \\
\hline
\end{tabular}


Table 21. Statistical summary of water-quality data for the upper Clark Fork basin, Montana, March 1985 through September 1999

[Abbreviations: $\mathrm{ft}^{3} / \mathrm{s}$, cubic feet per second; ${ }^{\circ} \mathrm{C}$, degrees Celsius; $\mu \mathrm{g} / \mathrm{L}$, micrograms per liter, $\mu \mathrm{S} / \mathrm{cm}$, microsiemens per centimeter at $25^{\circ} \mathrm{C} ; \mathrm{mg} / \mathrm{L}$, milligrams per liter; mm, millimeter; ton/d, tons per day. Symbols: <, less than laboratory reporting level ${ }^{1} ;--$, indicates insufficient data greater than minimum reporting level to compute statistic]

\begin{tabular}{|c|c|c|c|c|c|}
\hline Property or constituent and reporting unit & $\begin{array}{c}\text { Number of } \\
\text { samples }\end{array}$ & Maximum & Minimum & Mean & Median \\
\hline \multicolumn{6}{|c|}{$\begin{array}{l}\text { 12323230-BLACKTAIL CREEK AT HARRISON AVENUE, AT BUTTE, MONT. } \\
\text { Period of record for water-quality data: March 1993-August 1995, December 1996-September } 1999\end{array}$} \\
\hline Streamflow, instantaneous $\left(\mathrm{ft}^{3} / \mathrm{s}\right)$ & 51 & 156 & 3.1 & 19 & 9.3 \\
\hline Specific conductance, onsite $(\mu \mathrm{S} / \mathrm{cm})$ & 51 & 412 & 116 & 251 & 245 \\
\hline Temperature, water $\left({ }^{\circ} \mathrm{C}\right)$ & 51 & 17.0 & 2.0 & 8.0 & 7.0 \\
\hline pH, onsite (standard units) & 51 & 8.2 & 7.3 & 7.8 & 7.8 \\
\hline Hardness, total (mg/L as $\mathrm{CaCO}_{3}$ ) & 51 & 140 & 38 & 100 & 100 \\
\hline Calcium, dissolved (mg/L) & 51 & 39 & 11 & 28 & 29 \\
\hline Magnesium, dissolved (mg/L) & 51 & 11 & 2.7 & 6.9 & 6.7 \\
\hline Arsenic, total recoverable $(\mu \mathrm{g} / \mathrm{L})$ & 51 & 18 & 2 & 7 & 6 \\
\hline Arsenic, dissolved $(\mu \mathrm{g} / \mathrm{L})$ & 51 & 13 & 1 & 4 & 4 \\
\hline Cadmium, total recoverable $(\mu \mathrm{g} / \mathrm{L})$ & 51 & $<1$ & $<1$ & -- & $<1$ \\
\hline Cadmium, dissolved $(\mu \mathrm{g} / \mathrm{L})$ & 51 & .5 & $<.1$ & - & $<.1$ \\
\hline Copper, total recoverable $(\mu \mathrm{g} / \mathrm{L})$ & 51 & 52 & 2 & 8 & 6 \\
\hline Copper, dissolved $(\mu \mathrm{g} / \mathrm{L})$ & 51 & 10 & $<1$ & 24 & 4 \\
\hline Iron, total recoverable $(\mu \mathrm{g} / \mathrm{L})$ & 51 & 4,200 & 250 & 858 & 620 \\
\hline Iron, dissolved $(\mu \mathrm{g} / \mathrm{L})$ & 51 & 480 & 24 & 199 & 190 \\
\hline Lead, total recoverable $(\mu \mathrm{g} / \mathrm{L})$ & 51 & 47 & $<1$ & 23 & 1 \\
\hline Lead, dissolved $(\mu \mathrm{g} / \mathrm{L})$ & 51 & 1 & $<.5$ & ${ }^{2} .3$ & $<.5$ \\
\hline Manganese, total recoverable $(\mu \mathrm{g} / \mathrm{L})$ & 51 & 240 & 30 & 65 & 60 \\
\hline Manganese, dissolved $(\mu \mathrm{g} / \mathrm{L})$ & 51 & 100 & 17 & 42 & 39 \\
\hline Zinc, total recoverable $(\mu \mathrm{g} / \mathrm{L})$ & 51 & 130 & $<10$ & ${ }^{2} 12$ & $<10$ \\
\hline Zinc, dissolved $(\mu \mathrm{g} / \mathrm{L})$ & 51 & 11 & $<3$ & ${ }^{2} 4$ & $<20$ \\
\hline Sediment, suspended concentration $(\mathrm{mg} / \mathrm{L})$ & 51 & 139 & 2 & 19 & 8 \\
\hline Sediment, suspended discharge (ton/d) & 51 & 59 & .04 & 2.2 & .2 \\
\hline Sediment, suspended (percent finer than $0.062 \mathrm{~mm}$ ) & 51 & 97 & 50 & 83 & 86 \\
\hline
\end{tabular}


Table 21. Statistical summary of water-quality data for the upper Clark Fork basin, Montana, March 1985 through September 1999 (Continued)

\begin{tabular}{|c|c|c|c|c|c|}
\hline Property or constituent and reporting unit & $\begin{array}{l}\text { Number of } \\
\text { samples }\end{array}$ & Maximum & Minimum & Mean & Median \\
\hline \multicolumn{6}{|c|}{$\begin{array}{l}\text { 12323250-SILVER BOW CREEK BELOW BLACKTAIL CREEK, AT BUTTE, MONT. } \\
\text { Period of record for water-quality data: March 1993-August 1995, December 1996-September } 1999\end{array}$} \\
\hline Streamflow, instantaneous $\left(\mathrm{ft}^{3} / \mathrm{s}\right)$ & 51 & 134 & 15 & 35 & 29 \\
\hline Specific conductance, onsite $(\mu \mathrm{S} / \mathrm{cm})$ & 51 & 691 & 226 & 451 & 460 \\
\hline Temperature, water $\left({ }^{\circ} \mathrm{C}\right)$ & 51 & 17.0 & 1.5 & 9.9 & 9.0 \\
\hline pH, onsite (standard units) & 51 & 7.8 & 7.2 & 7.5 & 7.5 \\
\hline Hardness, total (mg/L as $\left.\mathrm{CaCO}_{3}\right)$ & 51 & 180 & 66 & 143 & 150 \\
\hline Calcium, dissolved $(\mathrm{mg} / \mathrm{L})$ & 51 & 52 & 19 & 41 & 42 \\
\hline Magnesium, dissolved (mg/L) & 51 & 13 & 4.5 & 10 & 10 \\
\hline Arsenic, total recoverable $(\mu \mathrm{g} / \mathrm{L})$ & 51 & 45 & 8 & 16 & 14 \\
\hline Arsenic, dissolved $(\mu \mathrm{g} / \mathrm{L})$ & 51 & 13 & 4 & 7 & 7 \\
\hline Cadmium, total recoverable $(\mu \mathrm{g} / \mathrm{L})$ & 51 & 6 & 1 & 3 & 2 \\
\hline Cadmium, dissolved $(\mu \mathrm{g} / \mathrm{L})$ & 51 & 6.2 & 5 & 2.1 & 1.6 \\
\hline Copper, total recoverable $(\mu \mathrm{g} / \mathrm{L})$ & 51 & 550 & 40 & 169 & 150 \\
\hline Copper, dissolved $(\mu \mathrm{g} / \mathrm{L})$ & 51 & 300 & 12 & 73 & 60 \\
\hline Iron, total recoverable $(\mu \mathrm{g} / \mathrm{L})$ & 51 & 7,400 & 250 & 1,480 & 820 \\
\hline Iron, dissolved $(\mu \mathrm{g} / \mathrm{L})$ & 51 & 270 & 20 & 108 & 100 \\
\hline Lead, total recoverable $(\mu \mathrm{g} / \mathrm{L})$ & 51 & 250 & 3 & 27 & 11 \\
\hline Lead, dissolved $(\mu \mathrm{g} / \mathrm{L})$ & 51 & 2.4 & $<.5$ & ${ }^{2} .8$ & .6 \\
\hline Manganese, total recoverable $(\mu \mathrm{g} / \mathrm{L})$ & 51 & 1,600 & 245 & 682 & 670 \\
\hline Manganese, dissolved $(\mu \mathrm{g} / \mathrm{L})$ & 51 & 1,700 & 210 & 619 & 630 \\
\hline Zinc, total recoverable $(\mu \mathrm{g} / \mathrm{L})$ & 51 & 2,200 & 254 & 856 & 790 \\
\hline Zinc, dissolved $(\mu \mathrm{g} / \mathrm{L})$ & 51 & 2,200 & 200 & 702 & 600 \\
\hline Sediment, suspended concentration (mg/L) & 50 & 405 & 3 & 40 & 16 \\
\hline Sediment, suspended discharge (ton/d) & 50 & 70 & .14 & 5.2 & 1.2 \\
\hline Sediment, suspended (percent finer than $0.062 \mathrm{~mm}$ ) & 50 & 98 & 42 & 83 & 86 \\
\hline
\end{tabular}


Table 21. Statistical summary of water-quality data for the upper Clark Fork basin, Montana, March 1985 through September 1999 (Continued)

\begin{tabular}{|c|c|c|c|c|c|}
\hline Property or constituent and reporting unit & $\begin{array}{c}\text { Number of } \\
\text { samples }\end{array}$ & Maximum & Minimum & Mean & Median \\
\hline \multicolumn{6}{|c|}{$\begin{array}{l}12323600-\text {-SILVER BOW CREEK AT OPPORTUNITY, MONT. } \\
\text { Period of record for water-quality data: March 1993-August 1995, December 1996-September } 1999\end{array}$} \\
\hline Streamflow, instantaneous $\left(\mathrm{ft}^{3} / \mathrm{s}\right)$ & 54 & 361 & 26 & 94 & 64 \\
\hline Specific conductance, onsite $(\mu \mathrm{S} / \mathrm{cm})$ & 53 & 593 & 202 & 368 & 368 \\
\hline Temperature, water $\left({ }^{\circ} \mathrm{C}\right)$ & 53 & 18.0 & 0.0 & 8.8 & 9.0 \\
\hline $\mathrm{pH}$, onsite (standard units) & 53 & 8.9 & 7.2 & 8.3 & 8.2 \\
\hline Hardness, total $\left(\mathrm{mg} / \mathrm{L}\right.$ as $\left.\mathrm{CaCO}_{3}\right)$ & 53 & 200 & 60 & 131 & 130 \\
\hline Calcium, dissolved $(\mathrm{mg} / \mathrm{L})$ & 53 & 55 & 18 & 39 & 39 \\
\hline Magnesium, dissolved (mg/L) & 53 & 15 & 3.4 & 8.3 & 8.2 \\
\hline Arsenic, total recoverable $(\mu \mathrm{g} / \mathrm{L})$ & 53 & 240 & 11 & 34 & 18 \\
\hline Arsenic, dissolved $(\mu \mathrm{g} / \mathrm{L})$ & 53 & 34 & $\mathbf{I}$ & 10 & 9 \\
\hline Cadmium, total recoverable $(\mu \mathrm{g} / \mathrm{L})$ & 53 & 49 & 1 & 3 & 2 \\
\hline Cadmium, dissolved $(\mu \mathrm{g} / \mathrm{L})$ & 53 & 41 & .1 & 2.0 & 1.2 \\
\hline Copper, total recoverable $(\mu \mathrm{g} / \mathrm{L})$ & 53 & 3,900 & 79 & 324 & 160 \\
\hline Copper, dissolved $(\mu \mathrm{g} / \mathrm{L})$ & 53 & 450 & 25 & 66 & 51 \\
\hline Iron, total recoverable $(\mu \mathrm{g} / \mathrm{L})$ & 53 & 24.000 & 290 & 2,230 & 990 \\
\hline Iron, dissolved $(\mu \mathrm{g} / \mathrm{L})$ & 53 & 310 & 3 & 59 & 33 \\
\hline Lead, total recoverable $(\mu \mathrm{g} / \mathrm{L})$ & 53 & 650 & 7 & 56 & 21 \\
\hline Lead, dissolved $(\mu \mathrm{g} / \mathrm{L})$ & 53 & 5.1 & $<.5$ & ${ }^{2} .8$ & .5 \\
\hline Manganese, total recoverable $(\mu \mathrm{g} / \mathrm{L})$ & 53 & 10,000 & 230 & 801 & 598 \\
\hline Manganese, dissolved $(\mu \mathrm{g} / \mathrm{L})$ & 53 & 9,300 & 190 & 660 & 490 \\
\hline Zinc, total recoverable $(\mu \mathrm{g} / \mathrm{L})$ & 53 & 15,000 & 230 & 866 & 533 \\
\hline Zinc, dissolved $(\mu \mathrm{g} / \mathrm{L})$ & 53 & 13,000 & 94 & 534 & 290 \\
\hline Sediment, suspended concentration $(\mathrm{mg} / \mathrm{L})$ & 54 & 801 & 6 & 76 & 24 \\
\hline Sediment, suspended discharge (ton/d) & 54 & 781 & 42 & 37 & 3.7 \\
\hline Sediment, suspended (percent finer than $0.062 \mathrm{~mm}$ ) & 54 & 95 & 37 & 76 & 79 \\
\hline
\end{tabular}


Table 21. Statistical summary of water-quality data for the upper Clark Fork basin, Montana, March 1985 through September 1999 (Continued)

\begin{tabular}{|c|c|c|c|c|c|}
\hline Property or constituent and reporting unit & $\begin{array}{l}\text { Number of } \\
\text { samples }\end{array}$ & Maximum & Minimum & Mean & Median \\
\hline \multicolumn{6}{|c|}{$\begin{array}{l}\text { 12323750--SILVER BOW CREEK AT WARM SPRINGS, MONT. } \\
\text { Period of record for water-quality data: March 1993-September } 1999\end{array}$} \\
\hline Streamflow, instantaneous $\left(\mathrm{ft}^{3} / \mathrm{s}\right)$ & 60 & 662 & 24 & 181 & 141 \\
\hline Specific conductance, onsite $(\mu \mathrm{S} / \mathrm{cm})$ & 58 & 614 & 249 & 430 & 426 \\
\hline Temperature, water $\left({ }^{\circ} \mathrm{C}\right)$ & 59 & 22.0 & .5 & 10.8 & 12.0 \\
\hline $\mathrm{pH}$, onsite (standard units) & 58 & 9.3 & 8.0 & 8.8 & 8.8 \\
\hline Hardness, total $\left(\mathrm{mg} / \mathrm{L}\right.$ as $\left.\mathrm{CaCO}_{3}\right)$ & 58 & 260 & 97 & 179 & 170 \\
\hline Calcium, dissolved (mg/L) & 58 & 78 & 28 & 52 & 50 \\
\hline Magnesium, dissolved (mg/L) & 58 & 19 & 5.9 & 12 & 11 \\
\hline Arsenic, total recoverable $(\mu \mathrm{g} / \mathrm{L})$ & 58 & 94 & 12 & 27 & 24 \\
\hline Arsenic, dissolved $(\mu \mathrm{g} / \mathrm{L})$ & 58 & 60 & 8 & 22 & 21 \\
\hline Cadmium, total recoverable $(\mu \mathrm{g} / \mathrm{L})$ & 58 & $<1$ & $<1$ & -- & $<1$ \\
\hline Cadmium, dissolved $(\mu \mathrm{g} / \mathrm{L})$ & 58 & 3 & $<.1$ & 2.1 & $<.1$ \\
\hline Copper, total recoverable $(\mu \mathrm{g} / \mathrm{L})$ & 58 & 80 & 5 & 25 & 21 \\
\hline Copper, dissolved $(\mu \mathrm{g} / \mathrm{L})$ & 58 & 40 & 3 & 12 & 10 \\
\hline Iron, total recoverable $(\mu \mathrm{g} / \mathrm{L})$ & 58 & 3,000 & 130 & 431 & 340 \\
\hline Iron, dissolved $(\mu \mathrm{g} / \mathrm{L})$ & 58 & 93 & $<5$ & ${ }^{2} 18$ & 15 \\
\hline Lead, total recoverable $(\mu \mathrm{g} / \mathrm{L})$ & 58 & 15 & $<1$ & $2_{3}$ & 2 \\
\hline Lead, dissolved $(\mu \mathrm{g} / \mathrm{L})$ & 58 & 1.0 & $<.5$ & - & $<.5$ \\
\hline Manganese, total recoverable $(\mu \mathrm{g} / \mathrm{L})$ & 58 & 600 & 60 & 192 & 155 \\
\hline Manganese, dissolved $(\mu \mathrm{g} / \mathrm{L})$ & 58 & 530 & 12 & 120 & 86 \\
\hline Zinc, total recoverable $(\mu \mathrm{g} / \mathrm{L})$ & 58 & 180 & $<10$ & ${ }^{2} 53$ & 40 \\
\hline Zinc, dissolved $(\mu \mathrm{g} / \mathrm{L})$ & 58 & 73 & $<3$ & ${ }^{2} 12$ & 7 \\
\hline Sediment, suspended concentration (mg/L) & 60 & 229 & 2 & 15 & 8 \\
\hline Sediment, suspended discharge (ton/d) & 60 & 279 & .26 & 12 & 2.6 \\
\hline Sediment, suspended (percent finer than $0.062 \mathrm{~mm}$ ) & 59 & 97 & 43 & 82 & 85 \\
\hline
\end{tabular}


Table 21. Statistical summary of water-quality data for the upper Clark Fork basin, Montana, March 1985 through September 1999 (Continued)

\begin{tabular}{|c|c|c|c|c|c|}
\hline Property or constituent and reporting unit & $\begin{array}{l}\text { Number of } \\
\text { samples }\end{array}$ & Maximum & Minimum & Mean & Median \\
\hline \multicolumn{6}{|c|}{$\frac{12323770 \text {--WARM SPRINGS CREEK AT WARM SPRINGS, MONT. }}{\text { Period of record for water quality data: March } 1993 \text {-September } 1999}$} \\
\hline Streamflow, instantaneous $\left(\mathrm{ft}^{3} / \mathrm{s}\right)$ & 40 & 420 & 2.8 & 124 & 95 \\
\hline Specific conductance, onsite $(\mu \mathrm{S} / \mathrm{cm})$ & 39 & 795 & 139 & 295 & 266 \\
\hline Temperature. water $\left({ }^{\circ} \mathrm{C}\right)$ & 40 & 16.0 & .5 & 8.6 & 9.0 \\
\hline pH, onsite (standard units) & 39 & 8.7 & 7.4 & 8.3 & 8.3 \\
\hline Hardness, total $\left(\mathrm{mg} / \mathrm{L}\right.$ as $\left.\mathrm{CaCO}_{3}\right)$ & 39 & 420 & 66 & 144 & 130 \\
\hline Calcium, dissolved (mg/L) & 39 & 130 & 20 & 44 & 39 \\
\hline Magnesium, dissolved (mg/L) & 39 & 22 & 3.6 & 8.3 & 7.3 \\
\hline Arsenic, total recoverable $(\mu \mathrm{g} / \mathrm{L})$ & 39 & 27 & 3 & 8 & 6 \\
\hline Arsenic, dissolved $(\mu \mathrm{g} / \mathrm{L})$ & 39 & 14 & 2 & 5 & 4 \\
\hline Cadmium, total recoverable $(\mu \mathrm{g} / \mathrm{L})$ & 39 & $<1$ & $<1$ & - & $<1$ \\
\hline Cadmium, dissolved $(\mu \mathrm{g} / \mathrm{L})$ & 39 & $<.1$ & $<.1$ & -- & $<1$ \\
\hline Copper, total recoverable $(\mu \mathrm{g} / \mathrm{L})$ & 39 & 97 & 2 & 25 & $1 \mathrm{I}$ \\
\hline Copper, dissolved $(\mu \mathrm{g} / \mathrm{L})$ & 39 & 16 & 1 & 4 & 3 \\
\hline Iron, total recoverable $(\mu \mathrm{g} / \mathrm{L})$ & 39 & 1,700 & 40 & 408 & 160 \\
\hline Iron, dissolved $(\mu \mathrm{g} / \mathrm{L})$ & 39 & 30 & 3 & 11 & 10 \\
\hline Lead, total recoverable $(\mu \mathrm{g} / \mathrm{L})$ & 39 & 14 & $<1$ & $2_{3}$ & 1 \\
\hline Lead, dissolved $(\mu \mathrm{g} / \mathrm{L})$ & 39 & 1.8 & $<.5$ & - & $<.5$ \\
\hline Manganese, total recoverable $(\mu \mathrm{g} / \mathrm{L})$ & 39 & 1,400 & 90 & 261 & 200 \\
\hline Manganese, dissolved ( $\mu \mathrm{g} / \mathrm{L})$ & 39 & 570 & 41 & 153 & 110 \\
\hline Zinc, total recoverable $(\mu \mathrm{g} / \mathrm{L})$ & 39 & 60 & $<10$ & ${ }^{2} 15$ & $<40$ \\
\hline Zinc, dissolved $(\mu \mathrm{g} / \mathrm{L})$ & 39 & 10 & $<1$ & $2_{3}$ & $<10$ \\
\hline Sediment, suspended concentration (mg/L) & 40 & 100 & 2 & 25 & 11 \\
\hline Sediment, suspended discharge (ton/d) & 39 & 87 & .09 & 14 & 3.0 \\
\hline Sediment, suspended (percent finer than $0.062 \mathrm{~mm}$ ) & 40 & 88 & 55 & 75 & 76 \\
\hline
\end{tabular}


Table 21. Statistical summary of water-quality data for the upper Clark Fork basin, Montana, March 1985 through September 1999 (Continued)

\begin{tabular}{|c|c|c|c|c|c|}
\hline Property or constituent and reporting unit & $\begin{array}{l}\text { Number of } \\
\text { samples }\end{array}$ & Maximum & Minimum & Mean & Median \\
\hline \multicolumn{6}{|c|}{$\begin{array}{l}\text { 12323800-CLARK FORK NEAR GALEN, MONT. } \\
\text { Period of record for water-quality data: July 1988-September } 1999\end{array}$} \\
\hline Streamflow, instantaneous $\left(\mathrm{ft}^{3} / \mathrm{s}\right)$ & 101 & 1,050 & 14 & 235 & 149 \\
\hline Specific conductance, onsite $(\mu \mathrm{S} / \mathrm{cm})$ & 89 & 720 & 197 & 422 & 423 \\
\hline Temperature, water $\left({ }^{\circ} \mathrm{C}\right)$ & 100 & 22.5 & 0.0 & 9.5 & 9.2 \\
\hline $\mathrm{pH}$, onsite (standard units) & 88 & 9.0 & 7.5 & 8.5 & 8.5 \\
\hline Hardness, total (mg/L as $\left.\mathrm{CaCO}_{3}\right)$ & 87 & 370 & 81 & 185 & 180 \\
\hline Calcium, dissolved $(\mathrm{mg} / \mathrm{L})$ & 87 & 110 & 24 & 55 & 54 \\
\hline Magnesium, dissolved (mg/L) & 87 & 22 & 5.1 & 12 & 11 \\
\hline Arsenic, total recoverable $(\mu \mathrm{g} / \mathrm{L})$ & 87 & 78 & 3 & 21 & 17 \\
\hline Arsenic, dissolved $(\mu \mathrm{g} / \mathrm{L})$ & 87 & 53 & 4 & 15 & 13 \\
\hline Cadmium, total recoverable $(\mu \mathrm{g} / \mathrm{L})$ & 87 & 3 & $<1$ & 2.3 & $<1$ \\
\hline Cadmium, dissolved $(\mu \mathrm{g} / \mathrm{L})$ & 87 & 1 & $<1$ & 2.1 & $<.1$ \\
\hline Copper, total recoverable $(\mu \mathrm{g} / \mathrm{L})$ & 86 & 240 & 5 & 38 & 24 \\
\hline Copper, dissolved $(\mu \mathrm{g} / \mathrm{L})$ & 87 & 50 & 3 & 11 & 9 \\
\hline Iron, total recoverable $(\mu \mathrm{g} / \mathrm{L})$ & 87 & 9,200 & 90 & 649 & 330 \\
\hline Iron, dissolved $(\mu \mathrm{g} / \mathrm{L})$ & 87 & 110 & $<3$ & ${ }^{2} 18$ & 12 \\
\hline Lead, total recoverable $(\mu \mathrm{g} / \mathrm{L})$ & 87 & 28 & $<1$ & 24 & 2 \\
\hline Lead, dissolved $(\mu \mathrm{g} / \mathrm{L})$ & 87 & 3 & $<.5$ & ${ }^{2}, 3$ & $<.5$ \\
\hline Manganese, total recoverable $(\mu \mathrm{g} / \mathrm{L})$ & 87 & 1,400 & 80 & 279 & 200 \\
\hline Manganese, dissolved $(\mu \mathrm{g} / \mathrm{L})$ & 87 & 380 & 31 & 121 & 89 \\
\hline Zinc, total recoverable $(\mu \mathrm{g} / \mathrm{L})$ & 87 & 360 & $<10$ & ${ }^{2} 59$ & 50 \\
\hline Zinc, dissolved $(\mu \mathrm{g} / \mathrm{L})$ & 87 & 110 & $<3$ & ${ }^{2} 15$ & 10 \\
\hline Sediment, suspended concentration (mg/L) & 101 & 338 & 2 & 23 & 10 \\
\hline Sediment, suspended discharge (ton/d) & 101 & 459 & 12 & 28 & 3.3 \\
\hline Sediment, suspended (percent finer than $0.062 \mathrm{~mm}$ ) & 100 & 97 & 41 & 78 & 78 \\
\hline
\end{tabular}


Table 21. Statistical summary of water-quality data for the upper Clark Fork basin, Montana, March 1985 through September 1999 (Continued)

\begin{tabular}{|c|c|c|c|c|c|}
\hline Property or constituent and reporting unit & $\begin{array}{l}\text { Number of } \\
\text { samples }\end{array}$ & Maximum & Minimum & Mean & Median \\
\hline \multicolumn{6}{|c|}{$\begin{array}{l}\text { 12324200-_CLARK FORK AT DEER LODGE, MONT. } \\
\text { Period of record for water-quality data: March 1985-September } 1999\end{array}$} \\
\hline Streamflow, instantaneous $\left(\mathrm{ft}^{3} / \mathrm{s}\right)$ & 153 & 1,920 & 23 & 313 & 231 \\
\hline Specific conductance, onsite $(\mu \mathrm{S} / \mathrm{cm})$ & 136 & 642 & 234 & 485 & 505 \\
\hline Temperature, water $\left({ }^{\circ} \mathrm{C}\right)$ & 152 & 23.0 & 0.0 & 9.4 & 100 \\
\hline $\mathrm{pH}$, onsite (standard units) & 101 & 8.9 & 7.4 & 8.2 & 8.2 \\
\hline Hardness, total (mg/L as $\left.\mathrm{CaCO}_{3}\right)$ & 93 & 270 & 95 & 202 & 210 \\
\hline Calcium, dissolved $(\mathrm{mg} / \mathrm{L})$ & 93 & 81 & 28 & 60 & 61 \\
\hline Magnesium, dissolved (mg/L) & 93 & 18 & 5.9 & 13 & 13 \\
\hline Arsenic, total recoverable $(\mu \mathrm{g} / \mathrm{L})$ & 103 & 220 & 8 & 28 & 18 \\
\hline Arsenic, dissolved $(\mu \mathrm{g} / \mathrm{L})$ & 103 & 39 & 7 & 15 & 13 \\
\hline Cadmium, total recoverable $(\mu \mathrm{g} / \mathrm{L})$ & 103 & 5 & $<1$ & ${ }^{2} .6$ & $<1$ \\
\hline Cadmium, dissolved $(\mu \mathrm{g} / \mathrm{L})$ & 103 & 2 & $<.1$ & 2.1 & $<1$ \\
\hline Copper, total recoverable $(\mu \mathrm{g} / \mathrm{L})$ & 102 & 1,500 & 10 & 110 & 51 \\
\hline Copper, dissolved ( $\mu \mathrm{g} / \mathrm{L})$ & 103 & 120 & 4 & 13 & 10 \\
\hline Iron, total recoverable $(\mu \mathrm{g} / \mathrm{L})$ & 103 & 29,000 & 60 & 2,150 & 780 \\
\hline Iron, dissolved $(\mu \mathrm{g} / \mathrm{L})$ & 103 & 190 & $<3$ & ${ }^{2} 17$ & 10 \\
\hline Lead, total recoverable $(\mu \mathrm{g} / \mathrm{L})$ & 103 & 200 & $<1$ & ${ }^{2} 14$ & 5 \\
\hline Lead, dissolved $(\mu \mathrm{g} / \mathrm{L})$ & 103 & 6 & $<.5$ & 2.5 & $<6$ \\
\hline Manganese, total recoverable $(\mu \mathrm{g} / \mathrm{L})$ & 103 & 4,600 & 30 & 319 & 180 \\
\hline Manganese, dissolved $(\mu \mathrm{g} / \mathrm{L})$ & 103 & 400 & 1 & 44 & 32 \\
\hline Zinc, total recoverable $(\mu \mathrm{g} / \mathrm{L})$ & 103 & 1,700 & 10 & 124 & 70 \\
\hline Zinc, dissolved $(\mu \mathrm{g} / \mathrm{L})$ & 103 & 230 & $<10$ & ${ }^{2} 16$ & 11 \\
\hline Sediment, suspended concentration $(\mathrm{mg} / \mathrm{L})$ & 153 & 2,250 & 2 & 89 & 25 \\
\hline Sediment, suspended discharge (ton/d) & 153 & 8,690 & .29 & 205 & 15 \\
\hline Sediment, suspended (percent finer than $0.062 \mathrm{~mm}$ ) & 144 & 99 & 40 & 70 & 70 \\
\hline
\end{tabular}


Table 21. Statistical summary of water-quality data for the upper Clark Fork basin, Montana, March 1985 through September 1999 (Continued)

\begin{tabular}{|c|c|c|c|c|c|}
\hline Property or constituent and reporting unit & $\begin{array}{l}\text { Number of } \\
\text { samples }\end{array}$ & Maximum & Minimum & Mean & Median \\
\hline \multicolumn{6}{|c|}{$\begin{array}{l}\text { 12324590-LITTLE BLACKFOOT RIVER NEAR GARRISON, MONT. } \\
\text { Period of record for water-quality data: March 1985-September 1999 }\end{array}$} \\
\hline Streamflow, instantaneous $\left(\mathrm{ft}^{3} / \mathrm{s}\right)$ & 84 & 2,080 & 21 & 313 & 198 \\
\hline Specific conductance, onsite $(\mu \mathrm{S} / \mathrm{cm})$ & 72 & 300 & 120 & 221 & 215 \\
\hline Temperature, water $\left({ }^{\circ} \mathrm{C}\right)$ & 83 & 22 & 0.0 & 8.1 & 8.0 \\
\hline $\mathrm{pH}$, onsite (standard units) & 71 & 8.5 & 7.0 & 8.0 & 8.1 \\
\hline Hardness, total (mg/L as $\left.\mathrm{CaCO}_{3}\right)$ & 66 & 140 & 51 & 101 & 99 \\
\hline Calcium, dissolved (mg/L) & 66 & 43 & 14 & 29 & 28 \\
\hline Magnesium, dissolved (mg/L) & 66 & 9.4 & 3.3 & 6.8 & 6.8 \\
\hline Arsenic, total recoverable $(\mu \mathrm{g} / \mathrm{L})$ & 71 & 17 & 4 & 7 & 6 \\
\hline Arsenic, dissolved $(\mu \mathrm{g} / \mathrm{L})$ & 71 & 7 & 3 & 5 & 5 \\
\hline Cadmium, total recoverable $(\mu \mathrm{g} / \mathrm{L})$ & 71 & 2 & $<1$ & 2.3 & $<1$ \\
\hline Cadmium, dissolved $(\mu \mathrm{g} / \mathrm{L})$ & 71 & 1 & $<.1$ & - & $<.1$ \\
\hline Copper, total recoverable $(\mu \mathrm{g} / \mathrm{L})$ & 70 & 45 & $<1$ & ${ }^{2} 5$ & 3 \\
\hline Copper, dissolved $(\mu \mathrm{g} / \mathrm{L})$ & 71 & 7 & $<1$ & ${ }^{2} 2$ & 2 \\
\hline Iron, total recoverable $(\mu \mathrm{g} / \mathrm{L})$ & 71 & 25.000 & 20 & 1,330 & 310 \\
\hline Iron, dissolved $(\mu \mathrm{g} / \mathrm{L})$ & 71 & 120 & $<3$ & ${ }^{2} 37$ & 27 \\
\hline Lead, total recoverable $(\mu \mathrm{g} / \mathrm{L})$ & 71 & 25 & $<1$ & ${ }^{2} 3$ & $<5$ \\
\hline Lead, dissolved $(\mu \mathrm{g} / \mathrm{L})$ & 70 & 6 & $<.5$ & ${ }^{2} .5$ & $<.6$ \\
\hline Manganese, total recoverable $(\mu \mathrm{g} / \mathrm{L})$ & 71 & 1,100 & $<10$ & ${ }^{2} 79$ & 30 \\
\hline Manganese, dissolved $(\mu \mathrm{g} / \mathrm{L})$ & 71 & 30 & 1 & 9 & 8 \\
\hline Zinc, total recoverable $(\mu \mathrm{g} / \mathrm{L})$ & 71 & 140 & $<10$ & ${ }^{2} 15$ & $<10$ \\
\hline Zinc, dissolved $(\mu \mathrm{g} / \mathrm{L})$ & 71 & 24 & $<1$ & $2_{4}$ & $<20$ \\
\hline Sediment, suspended concentration $(\mathrm{mg} / \mathrm{L})$ & 84 & 1,410 & 1 & 58 & 10 \\
\hline Sediment, suspended discharge (ton/d) & 84 & 7,920 & .08 & 163 & 6.6 \\
\hline Sediment, suspended (percent finer than $0.062 \mathrm{~mm}$ ) & 84 & 97 & 49 & 74 & 78 \\
\hline
\end{tabular}

68 Water-quality, bed-sediment, and biological data (October 1998 through September 1999) and statistical summaries of data for streams ia the Upper Clark Fork Basin, Montana 
Table 21. Statistical summary of water-quality data for the upper Clark Fork basin, Montana, March 1985 through September 1999 (Continued)

\begin{tabular}{|c|c|c|c|c|c|}
\hline Property or constituent and reporting unit & $\begin{array}{l}\text { Number of } \\
\text { samples }\end{array}$ & Maximum & Minimum & Mean & Median \\
\hline \multicolumn{6}{|c|}{$\begin{array}{l}\text { 12324680-CLARK FORK AT GOLDCREEK MONT. } \\
\text { Period of record for water-quality data: March 1993-September } 1999\end{array}$} \\
\hline Streamflow, instantaneous $\left(\mathrm{ft}^{3} / \mathrm{s}\right)$ & 59 & 3.920 & 87 & 902 & 620 \\
\hline Specific conductance, onsite $(\mu \mathrm{S} / \mathrm{cm})$ & 58 & 496 & 207 & 358 & 368 \\
\hline Temperature, water $\left({ }^{\circ} \mathrm{C}\right)$ & 59 & 20.0 & 0.0 & 9.1 & 9.5 \\
\hline $\mathrm{pH}$, onsite (standard units) & 58 & 8.7 & 7.9 & 8.3 & 8.3 \\
\hline Hardness, total (mg/L as $\left.\mathrm{CaCO}_{3}\right)$ & 58 & 230 & 86 & 157 & 160 \\
\hline Calcium, dissolved (mg/L) & 58 & 68 & 26 & 46 & 48 \\
\hline Magnesium, dissolved (mg/L) & 58 & 15 & 5.1 & 10 & 10 \\
\hline Arsenic, total recoverable ( $\mu \mathrm{g} / \mathrm{L})$ & 58 & 75 & 8 & 18 & 14 \\
\hline Arsenic, dissolved $(\mu \mathrm{g} / \mathrm{L})$ & 58 & 20 & 6 & 10 & 10 \\
\hline Cadmium, total recoverable $(\mu \mathrm{g} / \mathrm{L})$ & 58 & 2 & $<1$ & - & $<1$ \\
\hline Cadmium, dissolved $(\mu \mathrm{g} / \mathrm{L})$ & 58 & .2 & $<.1$ & - & $<.1$ \\
\hline Copper, total recoverable $(\mu \mathrm{g} / \mathrm{L})$ & 57 & 440 & 8 & 56 & 38 \\
\hline Copper, dissolved $(\mu \mathrm{g} / \mathrm{L})$ & 57 & 36 & 3 & 8 & 7 \\
\hline Iron, total recoverable $(\mu \mathrm{g} / \mathrm{L})$ & 58 & 12,000 & 60 & 1,220 & 680 \\
\hline Iron, dissolved $(\mu \mathrm{g} / \mathrm{L})$ & 58 & 100 & $<3$ & ${ }^{2} 21$ & 14 \\
\hline Lead, total recoverable $(\mu \mathrm{g} / \mathrm{L})$ & 57 & 73 & $<1$ & ${ }^{2} 8$ & 5 \\
\hline Lead, dissolved $(\mu \mathrm{g} / \mathrm{L})$ & 57 & .8 & $<.5$ & - & $<5$ \\
\hline Manganese, total recoverable $(\mu \mathrm{g} / \mathrm{L})$ & 58 & 1,100 & 30 & 157 & 116 \\
\hline Manganese, dissolved $(\mu \mathrm{g} / \mathrm{L})$ & 58 & 43 & 10 & 21 & 20 \\
\hline Zinc. total recoverable $(\mu \mathrm{g} / \mathrm{L})$ & 58 & 510 & 10 & 65 & 49 \\
\hline Zinc, dissolved $(\mu \mathrm{g} / \mathrm{L})$ & 58 & 26 & $<3$ & ${ }^{2} 8$ & 5 \\
\hline Sediment, suspended concentration ( $\mathrm{mg} / \mathrm{L}$ ) & 59 & 752 & 2 & 71 & 28 \\
\hline Sediment, suspended discharge (ton/d) & 59 & 7,960 & .94 & 360 & 47 \\
\hline Sediment, suspended (percent finer than $0.062 \mathrm{~mm}$ ) & 59 & 93 & 43 & 74 & 78 \\
\hline
\end{tabular}


Table 21. Statistical summary of water-quality data for the upper Clark Fork basin, Montana, March 1985 through September 1999 (Continued)

\begin{tabular}{|c|c|c|c|c|c|}
\hline Property or constituent and reporting unit & $\begin{array}{c}\text { Number of } \\
\text { samples }\end{array}$ & Maximum & Minimum & Mean & Median \\
\hline \multicolumn{6}{|c|}{$\begin{array}{l}\text { 12331500-FLINT CREEK NEAR DRUMMOND, MONT. } \\
\text { Period of record for water-quality data: March 1985-September } 1999\end{array}$} \\
\hline Streamflow, instantaneous $\left(\mathrm{ft}^{3} / \mathrm{s}\right)$ & 108 & 892 & 4.2 & 203 & 138 \\
\hline Specific conductance, onsite $(\mu \mathrm{S} / \mathrm{cm})$ & 97 & 507 & 134 & 289 & 290 \\
\hline Temperature, water $\left({ }^{\circ} \mathrm{C}\right)$ & 106 & 21.0 & 0.0 & 8.7 & 9.0 \\
\hline $\mathrm{pH}$, onsite (standard units) & 94 & 8.8 & 7.5 & 8.3 & 8.3 \\
\hline Hardness, total $\left(\mathrm{mg} / \mathrm{L}\right.$ as $\left.\mathrm{CaCO}_{3}\right)$ & 87 & 260 & 59 & 136 & 140 \\
\hline Calcium, dissolved (mg/L) & 87 & 73 & 16 & 37 & 37 \\
\hline Magnesium, dissolved (mg/L) & 87 & 20 & 4.3 & 11 & 11 \\
\hline Arsenic, total recoverable $(\mu \mathrm{g} / \mathrm{L})$ & 94 & 57 & 7 & 19 & 15 \\
\hline Arsenic, dissolved $(\mu \mathrm{g} / \mathrm{L})$ & 94 & 20 & 5 & 9 & 8 \\
\hline Cadmium, total recoverable $(\mu \mathrm{g} / \mathrm{L})$ & 94 & 3 & $<1$ & ${ }^{2} .2$ & $<1$ \\
\hline Cadmium, dissolved $(\mu \mathrm{g} / \mathrm{L})$ & 94 & .1 & $<.1$ & -- & $<.1$ \\
\hline Copper, total recoverable $(\mu \mathrm{g} / \mathrm{L})$ & 93 & 32 & 1 & 7 & 5 \\
\hline Copper, dissolved $(\mu \mathrm{g} / \mathrm{L})$ & 94 & 7 & $<1$ & ${ }^{2} 2$ & 2 \\
\hline Iron, total recoverable $(\mu \mathrm{g} / \mathrm{L})$ & 94 & 7,200 & 70 & 1,020 & 572 \\
\hline Iron, dissolved $(\mu \mathrm{g} / \mathrm{L})$ & 94 & 240 & 3 & 43 & 28 \\
\hline Lead, total recoverable $(\mu \mathrm{g} / \mathrm{L})$ & 94 & 87 & $<1$ & ${ }^{2} 13$ & 8 \\
\hline Lead, dissolved $(\mu \mathrm{g} / \mathrm{L})$ & 94 & 7 & $<.5$ & ${ }^{2} .8$ & $<1$ \\
\hline Manganese, total recoverable $(\mu \mathrm{g} / \mathrm{L})$ & 94 & 1,600 & 50 & 227 & 150 \\
\hline Manganese, dissolved $(\mu \mathrm{g} / \mathrm{L})$ & 94 & 120 & 14 & 39 & 34 \\
\hline Zinc, total recoverable $(\mu \mathrm{g} / \mathrm{L})$ & 94 & 290 & $<10$ & ${ }^{2} 43$ & 30 \\
\hline Zinc, dissolved $(\mu \mathrm{g} / \mathrm{L})$ & 94 & 27 & $<3$ & ${ }^{2} 6$ & 3 \\
\hline Sediment, suspended concentration ( $\mathrm{mg} / \mathrm{L}$ ) & 108 & 556 & 3 & 55 & 30 \\
\hline Sediment, suspended discharge (ton/d) & 106 & 904 & .03 & 51 & 9.6 \\
\hline Sediment, suspended (percent finer than $0.062 \mathrm{~mm}$ ) & 108 & 98 & 28 & 80 & 84 \\
\hline
\end{tabular}


Table 21. Statistical summary of water-quality data for the upper Clark Fork basin, Montana, March 1985 through September 1999 (Continued)

\begin{tabular}{|c|c|c|c|c|c|}
\hline Property or constituent and reporting unit & $\begin{array}{l}\text { Number of } \\
\text { samples }\end{array}$ & Maximum & Minimum & Mean & Median \\
\hline \multicolumn{6}{|c|}{$\begin{array}{l}\text { 12331800-CLARK FORK NEAR DRUMMOND, MONT. } \\
\text { Period of record for water-quality data: March 1993-September } 1999\end{array}$} \\
\hline Streamflow, instantaneous $\left(\mathrm{ft}^{3} / \mathrm{s}\right)$ & 59 & 3,860 & 149 & 1,260 & 907 \\
\hline Specific conductance, onsite $(\mu \mathrm{S} / \mathrm{cm})$ & 58 & 630 & 189 & 384 & 392 \\
\hline Temperature, water $\left({ }^{\circ} \mathrm{C}\right)$ & 59 & 21.0 & .5 & 10.1 & 11.0 \\
\hline $\mathrm{pH}$, onsite (standard units) & 58 & 8.5 & 7.8 & 8.3 & 8.3 \\
\hline Hardness, total (mg/L as $\left.\mathrm{CaCO}_{3}\right)$ & 58 & 300 & 74 & 173 & 180 \\
\hline Calcium, dissolved (mg/L) & 58 & 83 & 21 & 50 & 52 \\
\hline Magnesium, dissolved (mg/L) & 58 & 22 & 5.2 & 12 & 12 \\
\hline Arsenic, total recoverable $(\mu \mathrm{g} / \mathrm{L})$ & 58 & 62 & 8 & 19 & 16 \\
\hline Arsenic, dissolved $(\mu \mathrm{g} / \mathrm{L})$ & 58 & 20 & 7 & 11 & 10 \\
\hline Cadmium, total recoverable $(\mu \mathrm{g} / \mathrm{L})$ & 58 & 2 & $<1$ & - & $<1$ \\
\hline Cadmium, dissolved $(\mu \mathrm{g} / \mathrm{L})$ & 58 & .2 & $<.1$ & - & $<1$ \\
\hline Copper, total recoverable $(\mu \mathrm{g} / \mathrm{L})$ & 56 & 360 & 5 & 56 & 33 \\
\hline Copper, dissolved $(\mu \mathrm{g} / \mathrm{L})$ & 56 & 21 & 1 & 7 & 6 \\
\hline Iron, total recoverable $(\mu \mathrm{g} / \mathrm{L})$ & 58 & 8,800 & 50 & 1,360 & 698 \\
\hline Iron, dissolved $(\mu \mathrm{g} / \mathrm{L})$ & 58 & 150 & $<3$ & ${ }^{2} 23$ & 11 \\
\hline Lead, total recoverable $(\mu \mathrm{g} / \mathrm{L})$ & 54 & 56 & $<1$ & ${ }^{2} 11$ & 5 \\
\hline Lead, dissolved $(\mu \mathrm{g} / \mathrm{L})$ & 54 & 1.2 & $<.5$ & ${ }^{2} .3$ & $<.5$ \\
\hline Manganese, total recoverable $(\mu \mathrm{g} / \mathrm{L})$ & 58 & 880 & 20 & 184 & 129 \\
\hline Manganese, dissolved $(\mu \mathrm{g} / \mathrm{L})$ & 58 & 50 & 8 & 18 & 15 \\
\hline Zinc, total recoverable $(\mu \mathrm{g} / \mathrm{L})$ & 58 & 490 & $<10$ & ${ }^{2} 85$ & 50 \\
\hline Zinc, dissolved $(\mu \mathrm{g} / \mathrm{L})$ & 58 & 21 & $<3$ & ${ }^{2} 8$ & 6 \\
\hline Sediment, suspended concentration $(\mathrm{mg} / \mathrm{L})$ & 59 & 530 & 2 & 85 & 40 \\
\hline Sediment, suspended discharge (ton/d) & 59 & 4,720 & 1.9 & 475 & 103 \\
\hline Sediment, suspended (percent finer than $0.062 \mathrm{~mm}$ ) & 59 & 91 & 38 & 73 & 74 \\
\hline
\end{tabular}


Table 21. Statistical summary of water-quality data for the upper Clark Fork basin, Montana, March 1985 through September 1999 (Continued)

\begin{tabular}{|c|c|c|c|c|c|}
\hline Property or constituent and reporting unit & $\begin{array}{l}\text { Number of } \\
\text { samples }\end{array}$ & Maximum & Minimum & Mean & Median \\
\hline \multicolumn{6}{|c|}{$\begin{array}{l}12334510-\text {-ROCK CREEK NEAR CLINTON, MONT. } \\
\text { Period of record for water-quality data: March 1985-September } 1999\end{array}$} \\
\hline Streamflow, instantaneous $\left(\mathrm{ft}^{3} / \mathrm{s}\right)$ & 83 & 5,060 & 113 & 1,060 & 660 \\
\hline Specific conductance, onsite $(\mu \mathrm{S} / \mathrm{cm})$ & 74 & 155 & 53 & 103 & 94 \\
\hline Temperature, water $\left({ }^{\circ} \mathrm{C}\right)$ & 83 & 18 & 0.0 & 8.0 & 8.0 \\
\hline $\mathrm{pH}$, onsite (standard units) & 73 & 8.8 & 6.9 & 7.9 & 7.9 \\
\hline Hardness, total ( $\mathrm{mg} / \mathrm{L}$ as $\left.\mathrm{CaCO}_{3}\right)$ & 65 & 90 & 22 & 48 & 43 \\
\hline Calcium, dissolved (mg/L) & 65 & 23 & 5.9 & 13 & 11 \\
\hline Magnesium, dissolved (mg/L) & 65 & 8.8 & 1.9 & 4.1 & 3.7 \\
\hline Arsenic, total recoverable $(\mu \mathrm{g} / \mathrm{L})$ & 71 & 3 & $<1$ & ${ }^{2} 9$ & $<1$ \\
\hline Arsenic, dissolved $(\mu \mathrm{g} / \mathrm{L})$ & 71 & 1 & $<1$ & ${ }^{2} 1$ & $<1$ \\
\hline Cadmium, total recoverable $(\mu \mathrm{g} / \mathrm{L})$ & 71 & 3 & $<1$ & ${ }^{2} .4$ & $<1$ \\
\hline Cadmium, dissolved $(\mu \mathrm{g} / \mathrm{L})$ & 71 & 1 & $<.1$ & -- & $<.1$ \\
\hline Copper, total recoverable $(\mu \mathrm{g} / \mathrm{L})$ & 69 & 41 & $<1$ & 24 & 2 \\
\hline Copper, dissolved $(\mu \mathrm{g} / \mathrm{L})$ & 70 & 6 & $<1$ & 21 & $<1$ \\
\hline Iron, total recoverable $(\mu \mathrm{g} / \mathrm{L})$ & 71 & 2,100 & 20 & 357 & 180 \\
\hline Iron, dissolved $(\mu \mathrm{g} / \mathrm{L})$ & 71 & 160 & 5 & 39 & 35 \\
\hline Lead, total recoverable $(\mu \mathrm{g} / \mathrm{L})$ & 69 & 19 & $<1$ & ${ }^{2} 2$ & $<5$ \\
\hline Lead, dissolved $(\mu \mathrm{g} / \mathrm{L})$ & 69 & 5 & $<.5$ & ${ }^{2} .6$ & $<.6$ \\
\hline Manganese, total recoverable $(\mu \mathrm{g} / \mathrm{L})$ & 71 & 90 & $<10$ & ${ }^{2} 18$ & 10 \\
\hline Manganese, dissolved $(\mu \mathrm{g} / \mathrm{L})$ & 71 & 8 & $<1$ & ${ }^{2} 2$ & 2 \\
\hline Zinc, total recoverable $(\mu \mathrm{g} / \mathrm{L})$ & 71 & 60 & $<10$ & 29 & $<10$ \\
\hline Zinc, dissolved $(\mu \mathrm{g} / \mathrm{L})$ & 71 & 15 & $<3$ & 23 & $<3$ \\
\hline Sediment, suspended concentration $(\mathrm{mg} / \mathrm{L}$ ) & 83 & 223 & 1 & 25 & 7 \\
\hline Sediment, suspended discharge (ton/d) & 83 & 3,050 & 31 & 172 & 16 \\
\hline Sediment, suspended (percent finer than $0.062 \mathrm{~mm}$ ) & 83 & 95 & 35 & 69 & 69 \\
\hline
\end{tabular}


Table 21. Statistical summary of water-quality data for the upper Clark Fork basin. Montana, March 1985 through September 1999 (Continued)

\begin{tabular}{|c|c|c|c|c|c|}
\hline Property or constituent and reporting unit & $\begin{array}{l}\text { Number of } \\
\text { samples }\end{array}$ & Maximum & Minimum & Mean & Median \\
\hline \multicolumn{6}{|c|}{$\frac{\text { 12334550-CLARK FORK AT TURAH BRIDGE, NEAR BONNER, MONT. }}{\text { Period of record for water-quality data: March 1985-September } 1999}$} \\
\hline Streamflow, instantaneous $\left(\mathrm{ft}^{3} / \mathrm{s}\right)$ & 156 & 9,560 & 296 & 1,950 & 1,170 \\
\hline Specific conductance, onsite $(\mu \mathrm{S} / \mathrm{cm})$ & 131 & 483 & 140 & 306 & 327 \\
\hline Temperature, water $\left({ }^{\circ} \mathrm{C}\right)$ & 155 & 22.0 & 0.0 & 9.1 & 9.5 \\
\hline pH, onsite (standard units) & 102 & 8.8 & 7.4 & 8.2 & 8.2 \\
\hline Hardness, total $\left(\mathrm{mg} / \mathrm{L}\right.$ as $\left.\mathrm{CaCO}_{3}\right)$ & 92 & 210 & 58 & 133 & 135 \\
\hline Calcium, dissolved $(\mathrm{mg} / \mathrm{L})$ & 92 & 59 & 16 & 37 & 38 \\
\hline Magnesium, dissolved (mg/L) & 92 & 14 & 3.9 & 9.4 & 9.4 \\
\hline Arsenic, total recoverable $(\mu \mathrm{g} / \mathrm{L})$ & 101 & 110 & 5 & 11 & 8 \\
\hline Arsenic, dissolved $(\mu \mathrm{g} / \mathrm{L})$ & 101 & 17 & 4 & 6 & 5 \\
\hline Cadmium, total recoverable $(\mu \mathrm{g} / \mathrm{L})$ & 101 & 4 & $<1$ & ${ }^{2} .4$ & $<1$ \\
\hline Cadmium, dissolved $(\mu \mathrm{g} / \mathrm{L})$ & 101 & 1 & $<.1$ & - & $<.1$ \\
\hline Copper, total recoverable $(\mu \mathrm{g} / \mathrm{L})$ & 99 & 500 & 3 & 46 & 23 \\
\hline Copper, dissolved $(\mu \mathrm{g} / \mathrm{L})$ & 100 & 25 & 2 & 6 & 4 \\
\hline Iron, total recoverable $(\mu \mathrm{g} / \mathrm{L})$ & 101 & 19,000 & 53 & 1,400 & 530 \\
\hline Iron, dissolved $(\mu \mathrm{g} / \mathrm{L})$ & 101 & 190 & $<3$ & ${ }^{2} 27$ & 16 \\
\hline Lead, total recoverable $(\mu \mathrm{g} / \mathrm{L})$ & 97 & 100 & $<1$ & ${ }^{2} 10$ & 5 \\
\hline Lead, dissolved $(\mu \mathrm{g} / \mathrm{L})$ & 97 & 7 & $<.5$ & ${ }^{2} .4$ & $<.6$ \\
\hline Manganese, total recoverable $(\mu \mathrm{g} / \mathrm{L})$ & 101 & 2,000 & 10 & 161 & 90 \\
\hline Manganese, dissolved $(\mu \mathrm{g} / \mathrm{L})$ & 101 & 37 & 1 & 9 & 7 \\
\hline Zinc, total recoverable $(\mu \mathrm{g} / \mathrm{L})$ & 101 & 1,100 & $<10$ & ${ }^{2} 83$ & 40 \\
\hline Zinc, dissolved $(\mu \mathrm{g} / \mathrm{L})$ & 101 & 39 & $<3$ & ${ }^{2} 8$ & 6 \\
\hline Sediment, suspended concentration (mg/L) & 156 & 1,370 & 2 & 67 & 23 \\
\hline Sediment, suspended discharge (ton/d) & 156 & 34,700 & 3.5 & 781 & 67 \\
\hline Sediment, suspended (percent finer than $0.062 \mathrm{~mm}$ ) & 145 & 98 & 27 & 72 & 72 \\
\hline
\end{tabular}


Table 21. Statistical summary of water-quality data for the upper Clark Fork basin, Montana, March 1985 through September 1999 (Continued)

\begin{tabular}{|c|c|c|c|c|c|}
\hline Property or constituent and reporting unit & $\begin{array}{l}\text { Number of } \\
\text { samples }\end{array}$ & Maximum & Minimum & Mean & Median \\
\hline \multicolumn{6}{|c|}{$\begin{array}{l}\text { 12340000-BLACKFOOT RIVER NEAR BONNER, MONT. } \\
\text { Period of record for water-quality data: March 1985-September } 1999\end{array}$} \\
\hline Streamflow, instantaneous $\left(\mathrm{ft}^{3} / \mathrm{s}\right)$ & 114 & 13,400 & 344 & 2,820 & 1,340 \\
\hline Specific conductance, onsite $(\mu \mathrm{S} / \mathrm{cm})$ & 91 & 294 & 130 & 204 & 200 \\
\hline Temperature, water $\left({ }^{\circ} \mathrm{C}\right)$ & 114 & 20.5 & 0.0 & 8.9 & 9.0 \\
\hline $\mathrm{pH}$, onsite (standard units) & 74 & 8.7 & 7.5 & 8.2 & 8.3 \\
\hline Hardness, total $\left(\mathrm{mg} / \mathrm{L}\right.$ as $\left.\mathrm{CaCO}_{3}\right)$ & 67 & 140 & 55 & 100 & 94 \\
\hline Calcium, dissolved (mg/L) & 67 & 37 & 14 & 26 & 24 \\
\hline Magnesium, dissolved (mg/L) & 67 & 13 & 4.9 & 8.8 & 8.3 \\
\hline Arsenic, total recoverable $(\mu \mathrm{g} / \mathrm{L})$ & 74 & 4 & $<1$ & ${ }^{2} 1$ & 1 \\
\hline Arsenic, dissolved $(\mu \mathrm{g} / \mathrm{L})$ & 74 & 2 & $<1$ & ${ }^{2} .9$ & $<1$ \\
\hline Cadmium, total recoverable $(\mu \mathrm{g} / \mathrm{L})$ & 74 & 2 & $<1$ & 2.4 & $<1$ \\
\hline Cadmium, dissolved $(\mu \mathrm{g} / \mathrm{L})$ & 74 & 1 & $<.1$ & - & $<.1$ \\
\hline Copper, total recoverable $(\mu \mathrm{g} / \mathrm{L})$ & 71 & 34 & $<1$ & ${ }^{2} 7$ & 5 \\
\hline Copper, dissolved $(\mu \mathrm{g} / \mathrm{L})$ & 72 & 7 & $<1$ & ${ }^{2} 2$ & 1 \\
\hline Iron, total recoverable $(\mu \mathrm{g} / \mathrm{L})$ & 74 & 3.600 & 20 & 583 & 250 \\
\hline Iron, dissolved $(\mu \mathrm{g} / \mathrm{L})$ & 74 & 100 & $<3$ & ${ }^{2} 20$ & 14 \\
\hline Lead, total recoverable $(\mu \mathrm{g} / \mathrm{L})$ & 70 & 25 & $<1$ & $2_{4}$ & 1 \\
\hline Lead. dissolved $(\mu \mathrm{g} / \mathrm{L})$ & 70 & 8 & $<.5$ & ${ }^{2} 1$ & $<1$ \\
\hline Manganese, total recoverable $(\mu \mathrm{g} / \mathrm{L})$ & 74 & 180 & $<10$ & ${ }^{2} 38$ & 20 \\
\hline Manganese, dissolved $(\mu \mathrm{g} / \mathrm{L})$ & 74 & 11 & $<1$ & ${ }^{2} 3$ & 2 \\
\hline Zinc, total recoverable $(\mu \mathrm{g} / \mathrm{L})$ & 74 & 60 & $<10$ & ${ }^{2} 10$ & $<10$ \\
\hline Zinc, dissolved $(\mu \mathrm{g} / \mathrm{L})$ & 74 & 15 & $<1$ & $2_{3}$ & $<10$ \\
\hline Sediment, suspended concentration ( $\mathrm{mg} / \mathrm{L}$ ) & 114 & 271 & 1 & 35 & 10 \\
\hline Sediment, suspended discharge (ton/d) & 114 & 7,670 & 1.1 & 642 & 31 \\
\hline Sediment, suspended (percent finer than $0.062 \mathrm{~mm}$ ) & 112 & 98 & 42 & 79 & 81 \\
\hline
\end{tabular}

74 Water-quality, bed-sediment, and biological data (October 1998 through September 1999) and statistical summaries of data for streams in the Upper Clark Fork Basin, Montana 
Table 21. Statistical summary of water-quality data for the upper Clark Fork basin, Montana, March 1985 through September 1999 (Continued)

\begin{tabular}{|c|c|c|c|c|c|}
\hline Property or constituent and reporting unit & $\begin{array}{l}\text { Number of } \\
\text { samples }\end{array}$ & Maximum & Minimum & Mean & Median \\
\hline \multicolumn{6}{|c|}{$\begin{array}{l}\text { 12340500-CLARK FORK ABOVE MISSOULA, MONT. } \\
\text { Period of record for water-quality data: July 1986-September } 1999\end{array}$} \\
\hline Streamflow, instantaneous $\left(\mathrm{ft}^{3} / \mathrm{s}\right)$ & 122 & 21,600 & 720 & 4,670 & 2.380 \\
\hline Specific conductance, onsite $(\mu \mathrm{S} / \mathrm{cm})$ & 99 & 399 & 142 & 254 & 261 \\
\hline Temperature. water $\left({ }^{\circ} \mathrm{C}\right)$ & 119 & 19.5 & 0.0 & 9.0 & 8.5 \\
\hline $\mathrm{pH}$, onsite (standard units) & 79 & 8.7 & 7.9 & 8.3 & 8.3 \\
\hline Hardness, total $\left(\mathrm{mg} / \mathrm{L}\right.$ as $\left.\mathrm{CaCO}_{3}\right)$ & 79 & 170 & 61 & 117 & 120 \\
\hline Calcium, dissolved (mg/L) & 79 & 46 & 14 & 31 & 32 \\
\hline Magnesium, dissolved (mg/L) & 79 & 13 & 5.3 & 9.2 & 9.2 \\
\hline Arsenic, total recoverable $(\mu \mathrm{g} / \mathrm{L})$ & 79 & 69 & 2 & 6 & 4 \\
\hline Arsenic, dissolved $(\mu \mathrm{g} / \mathrm{L})$ & 79 & 9 & 1 & 3 & 3 \\
\hline Cadmium, total recoverable $(\mu \mathrm{g} / \mathrm{L})$ & 79 & 5 & $<1$ & $\cdots$ & $<1$ \\
\hline Cadmium, dissolved $(\mu \mathrm{g} / \mathrm{L})$ & 79 & .1 & $<.1$ & -- & $<.1$ \\
\hline Copper, total recoverable $(\mu \mathrm{g} / \mathrm{L})$ & 77 & 400 & 2 & 19 & 8 \\
\hline Copper, dissolved $(\mu \mathrm{g} / \mathrm{L})$ & 78 & 11 & 1 & 3 & 2 \\
\hline Iron, total recoverable $(\mu \mathrm{g} / \mathrm{L})$ & 79 & 13,000 & 60 & 728 & 270 \\
\hline Iron, dissolved $(\mu \mathrm{g} / \mathrm{L})$ & 79 & 200 & $<3$ & ${ }^{2} 25$ & 16 \\
\hline Lead, total recoverable $(\mu \mathrm{g} / \mathrm{L})$ & 74 & 78 & $<1$ & ${ }^{2} 4$ & 2 \\
\hline Lead, dissolved $(\mu \mathrm{g} / \mathrm{L})$ & 74 & 1 & $<.5$ & ${ }^{2} .6$ & $<.5$ \\
\hline Manganese, total recoverable $(\mu \mathrm{g} / \mathrm{L})$ & 79 & 1,100 & 10 & 74 & 40 \\
\hline Manganese, dissolved $(\mu \mathrm{g} / \mathrm{L})$ & 79 & 230 & 7 & 19 & 15 \\
\hline Zinc, total recoverable $(\mu \mathrm{g} / \mathrm{L})$ & 79 & 1,100 & $<10$ & ${ }^{2} 37$ & 20 \\
\hline Zinc, dissolved $(\mu \mathrm{g} / \mathrm{L})$ & 79 & 16 & $<3$ & ${ }^{2} 5$ & 3 \\
\hline Sediment, suspended concentration (mg/L) & 122 & 824 & 2 & 41 & 12 \\
\hline Sediment, suspended discharge (ton/d) & 122 & 21,900 & 6.1 & 1,130 & 66 \\
\hline Sediment, suspended (percent finer than $0.062 \mathrm{~mm}$ ) & 117 & 99 & 44 & 86 & 90 \\
\hline
\end{tabular}

${ }^{1}$ Multiple less-than (<) values for an individual constituent are the result of changes in analytical laboratory reporting levels during the period of record

${ }^{2}$ Value is estimated by using a log-probability regression to predict the values of data less than the laboratory reporting level (Helsel and Cohn, 1988). 
Table 22. Statistical summary of fine-grained bed-sediment data for the upper Clark Fork basin, Montana, August 1986 through August 1999

[Fine-grained bed sediment is material less than 0.064 millimeter in diameter. Reported concentrations are in micrograms per gram dry

weight. Symbols: <, less than minimum reporting level; --, indicates insufficient data greater than the minimum reporting level to compute statistic. Number of samples represents the number of years that the constituent was analyzed, with each year represented by a single mean concentration of composite samples]

\begin{tabular}{|c|c|c|c|c|c|}
\hline Constituent & $\begin{array}{c}\text { Number } \\
\text { of } \\
\text { samples }\end{array}$ & $\begin{array}{l}\text { Maxi- } \\
\text { mum }\end{array}$ & Minimum & Mean & Median \\
\hline \multicolumn{6}{|c|}{$\frac{12323600-\text { SILVER BOW CREEK AT OPPORTUNITY, MONT. }}{\text { Period of record for fine-grained bed-sediment data: } 1992-99}$} \\
\hline Cadmium & 8 & 42.0 & 23.7 & 31.1 & 28.2 \\
\hline Chromium & 7 & 32.4 & 23.2 & 27.6 & 27.6 \\
\hline Copper & 8 & 6,280 & 4,220 & 5,010 & 4,740 \\
\hline Iron & 8 & 41,200 & 34,400 & 38,400 & 39.000 \\
\hline Lead & 8 & 1,030 & 752 & 848 & 833 \\
\hline Manganese & 8 & 3,940 & 1.680 & 2,540 & 2,440 \\
\hline Nickel & 7 & 21.4 & 14.2 & 16.4 & 16.1 \\
\hline Silver & 8 & 19.6 & 13.7 & 16.6 & 16.8 \\
\hline Zinc & 8 & 10,800 & 6,660 & 8,060 & 7,570 \\
\hline
\end{tabular}

Cadmium
Chromium
Copper
Iron
Lead
Manganese
Nickel
Silver
Zinc

Cadmium

Chromium

Copper

Iron

Lead

Manganese

Nickel

Silver

Zinc

\section{0--SILVER BOW CREEK AT WARM SPRINGS, MONT.}

Period of record for fine-grained bed-sediment data: 1992-99

$\begin{array}{lcccc}\text { for fine-grained bed-sediment data: } \mathbf{1 9 9 2 - 9 9} & \\ 8 & 12.2 & 4.5 & 7.5 & 6.7 \\ 7 & 34.1 & 12.8 & 22.7 & 23.6 \\ 8 & 769 & 223 & 434 & 351 \\ 8 & 27,200 & 19,500 & 22,100 & 20,800 \\ 8 & 100 & 58 & 76 & 74 \\ 8 & 17,700 & 1,470 & 6,480 & 5,590 \\ 7 & 19.1 & 12.5 & 15.5 & 15.7 \\ 8 & 2.1 & .3 & 11.4 & 1.4 \\ 8 & 2,220 & 620 & 1,160 & 830\end{array}$

12323770--WARM SPRINGS CREEK AT WARM SPRINGS, MONT.

Period of record for fine-grained bed-sediment data: 1995,1997 
Table 22. Statistical summary of fine-grained bed-sediment data for the upper Clark Fork basin, Montana, August 1986 through August 1999 (Continued)

\begin{tabular}{|c|c|c|c|c|c|}
\hline Constituent & $\begin{array}{c}\text { Number } \\
\text { of } \\
\text { samples }\end{array}$ & $\begin{array}{l}\text { Maxi- } \\
\text { mum }\end{array}$ & Minimum & Mean & Median \\
\hline \multicolumn{6}{|c|}{$\begin{array}{l}\text { 12323800-CLARK FORK NEAR GALEN, MONT. } \\
\text { of record for fine-grained bed-sediment data: } 1987,1991-99\end{array}$} \\
\hline Cadmium & 10 & 20.1 & 4.0 & 10.4 & 9.2 \\
\hline Chromium & 7 & 33.9 & 22.1 & 28.3 & 29.9 \\
\hline Copper & 10 & 2,300 & 991 & 1,350 & 1,230 \\
\hline Iron & 10 & 39,800 & 22,600 & 29,600 & 28,400 \\
\hline Lead & 10 & 235 & 116 & 152 & 144 \\
\hline Manganese & 10 & 15,600 & 2,780 & 8,460 & 7,440 \\
\hline Nickel & 7 & 23.2 & 17.7 & 19.8 & 19.3 \\
\hline Silver & 10 & 5.5 & $<3.2$ & $1_{3.8}$ & ${ }^{1} 3.7$ \\
\hline Zinc & 10 & 3,560 & 1,120 & 1,920 & 1,600 \\
\hline
\end{tabular}

461415112450801--CLARK FORK BELOW LOST CREEK, NEAR GALEN, MONT Period of record for fine-grained bed-sediment data: 1996-99

Cadmium

Chromium

Copper

Iron

Lead

Manganese

Nickel

Silver

Zinc

Cadmium
Chromium
Copper
Iron
Lead
Manganese
Nickel
Silver
Zinc

461559112443301--CLARK FORK NEAR RACETRACK, MONT. Period of record for fine-grained bed-sediment data: 1996-99

$\begin{array}{rccc}9.0 & 6.5 & 7.7 & 7.6 \\ 34.5 & 27.5 & 31.7 & 32.5 \\ 2,050 & 1,360 & 1,650 & 1,580 \\ 32,700 & 30,800 & 31,600 & 31,600 \\ 197 & 168 & 182 & 182 \\ 5,900 & 3,540 & 4,600 & 4,490 \\ 19.9 & 17.8 & 18.8 & 18.7 \\ 7.0 & 4.2 & 5.9 & 6.2 \\ 1,680 & 1,280 & 1,480 & 1,470\end{array}$

$\begin{array}{rccc}8.5 & 5.0 & 6.6 & 6.5 \\ 33.3 & 24.9 & 29.6 & 30.0 \\ 1,610 & 946 & 1,330 & 1,380 \\ 31,700 & 28,600 & 29,800 & 29,400 \\ 186 & 134 & 157 & 154 \\ 4,030 & 2,390 & 3,060 & 2.900 \\ 18.4 & 16.5 & 17.1 & 16.8 \\ 6.1 & <3.3 & 14.6 & 15.4 \\ 1,550 & 1,030 & 1,300 & 1,310\end{array}$

\begin{tabular}{|c|c|c|c|c|c|}
\hline \multicolumn{6}{|c|}{ Period of record for fine-grained bed-sediment data: $1996-99$} \\
\hline Cadmium & 4 & 8.1 & 4.3 & 6.0 & 5.9 \\
\hline Chromium & 4 & 34.1 & 26.4 & 30.1 & 30.0 \\
\hline Copper & 4 & 1,550 & 766 & 1,110 & 1.060 \\
\hline Iron & 4 & 33,700 & 28,200 & 30,100 & 29.200 \\
\hline Lead & 4 & 152 & 115 & 134 & 134 \\
\hline Manganese & 4 & 3,910 & 1,810 & 2,760 & 2,660 \\
\hline Nickel & 4 & 16.9 & 15.1 & 16.1 & 16.3 \\
\hline Silver & 4 & 6.2 & 2.7 & 4.7 & 4.9 \\
\hline Zinc & 4 & 1,570 & 900 & 1,160 & 1,080 \\
\hline
\end{tabular}


Table 22. Statistical summary of fine-grained bed-sediment data for the upper Clark Fork basin, Montana, August 1986 through August 1999 (Continued)

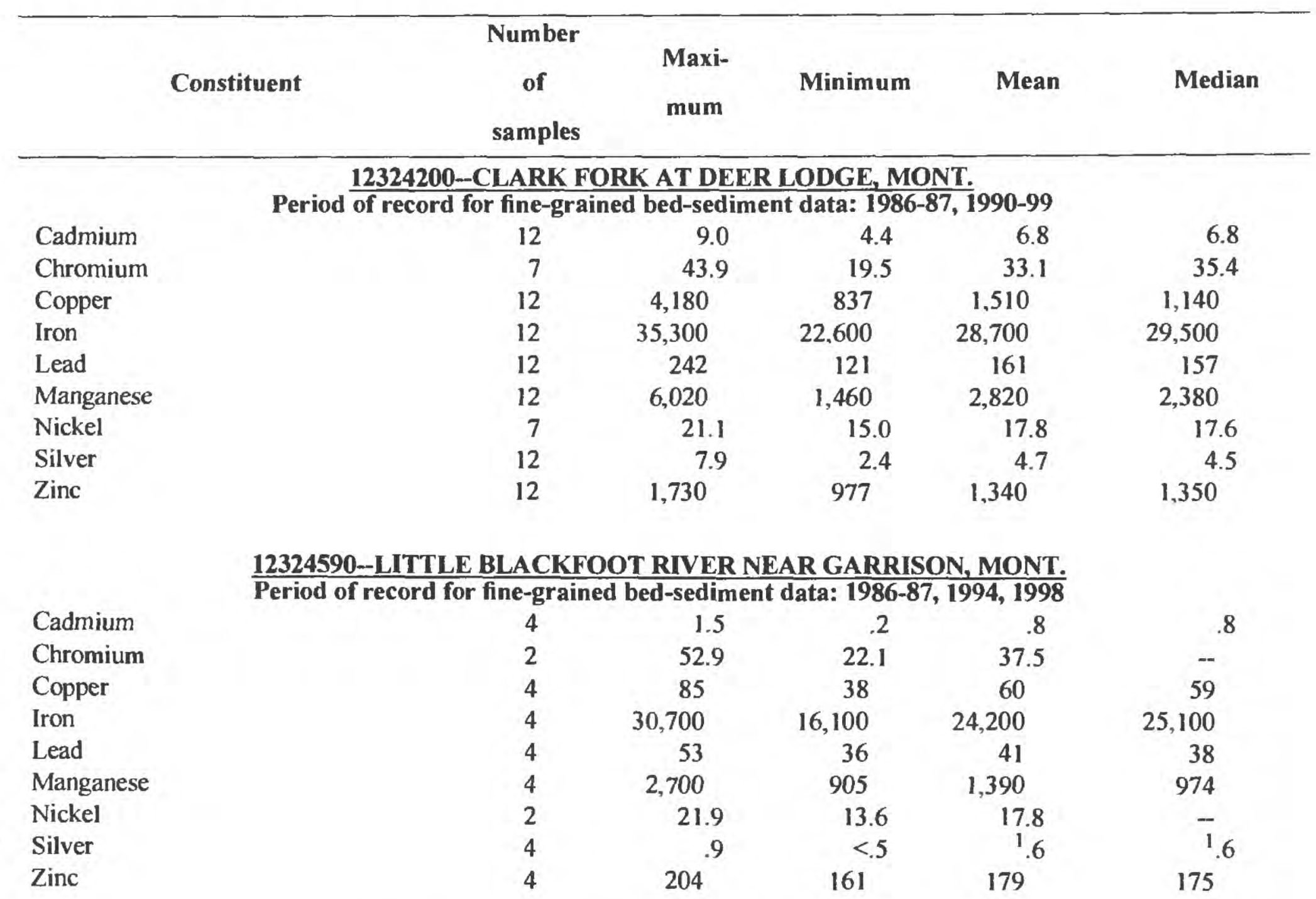

12324680-CLARK FORK AT GOLDCREEK, MONT,

\begin{tabular}{lcccrr}
\multicolumn{7}{c}{ Period of record for fine-grained bed-sediment data: 1992-99 } \\
Cadmium & 8 & 6.2 & 3.5 & 5.4 & 5.7 \\
Chromium & 7 & 48.9 & 29.4 & 35.8 & 34.5 \\
Copper & 8 & 1,080 & 653 & 833 & 786 \\
Iron & 8 & 30,600 & 20,500 & 25,900 & 25,800 \\
Lead & 8 & 152 & 88 & 114 & 119 \\
Manganese & 8 & 2,610 & 1,180 & 1.940 & 1,810 \\
Nickel & 7 & 18.6 & 15.0 & 16.8 & 17.0 \\
Silver & 8 & 4.8 & 2.3 & 3.5 & 3.5 \\
Zinc & 8 & 1,320 & 1,070 & 1,160 & 1,140
\end{tabular}

12331500--FLINT CREEK NEAR DRUMMOND, MONT.

$\begin{array}{lccccc}\text { Cadmium } & \text { Period of record for fine-grained bed-sediment data: } \mathbf{1 9 8 6 , 1 9 8 9 ,} \text { 1992-99 } & 1_{2.4} \\ \text { Chromium } & 10 & 4.5 & <.2 & 12.3 & 24.3 \\ \text { Copper } & 7 & 29.2 & 20.4 & 24.7 & 63 \\ \text { Iron } & 10 & 73 & 55 & 62 & 23,500 \\ \text { Lead } & 10 & 28,100 & 21,100 & 24.100 & 176 \\ \text { Manganese } & 10 & 240 & 150 & 180 & 3,740 \\ \text { Nickel } & 10 & 5,510 & 2,370 & 3,790 & 12.7 \\ \text { Silver } & 7 & 14.9 & 11.7 & 13.1 & 6.4 \\ \text { Zinc } & 9 & 7.8 & 5.0 & 6.3 & 661\end{array}$


Table 22. Statistical summary of fine-grained bed-sediment data for the upper Clark Fork basin, Montana, August 1986 through August 1999 (Continued)

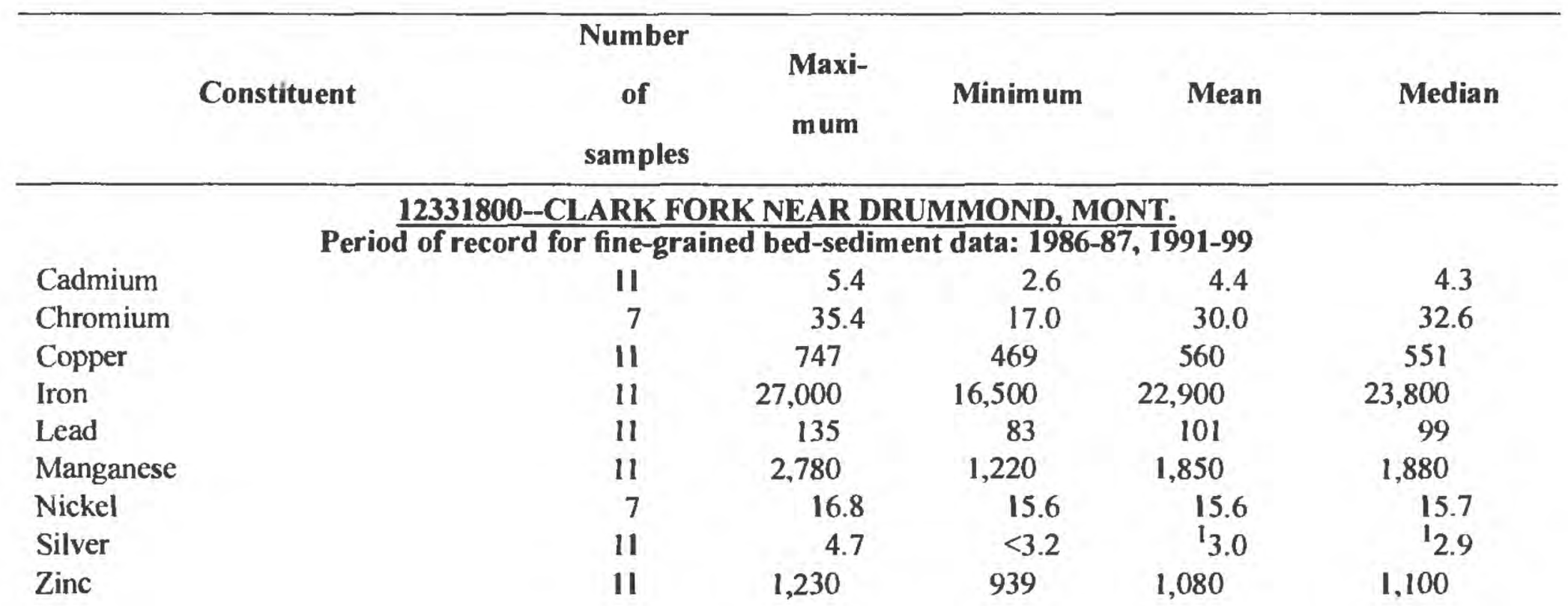

12334510--ROCK CREEK NEAR CLINTON, MONT.

Cadmium

Chromium

Copper

Iron

Lead

Manganese

Nickel

Silver

Zinc

\section{Cadmium}

Chromium

Copper

Iron

Lead

Manganese

Nickel

Silver

Zinc

Cadmium

Chromium

Copper

Iron

Lead

Manganese

Nickel

Silver

Zinc
Period of record for fine-grained bed-sediment data: 1986-87, 1989, 1991-99

12
7
12
12
12
12
7
11
12

$<1.5$
27.9
16
21,400
16
724
14.8
.8
58

$<.2$
16.5
3
13,100
$<3$
126
10.8
$<.3$
36

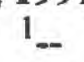

22.6

12

18,100

18

405

12.9

1.4

48

\section{0--CLARK FORK AT TURAH BRIDGE, NEAR BONNER, MONT.}

Period of record for fine-grained bed-sediment data: 1986, 1991-99

$\begin{array}{rcccc}10 & 5.2 & 3.1 & 3.8 & 3.7 \\ 7 & 34.7 & 15.3 & 26.4 & 27.7 \\ 10 & 635 & 300 & 420 & 384 \\ 10 & 24,400 & 15,100 & 20,400 & 22,000 \\ 10 & 115 & 49 & 80 & 80 \\ 10 & 2,270 & 671 & 1,260 & 1,200 \\ 7 & 19.1 & 11.6 & 15.3 & 16.2 \\ 10 & 3.9 & 1.0 & 2.2 & 2.2 \\ 10 & 1,160 & 775 & 941 & 913\end{array}$

12340000--BLACKFOOT RIVER NEAR BONNER, MONT.

Period of record for fine-grained bed-sediment data: 1986-87, 1991, 1993-96, 1998-99

\begin{tabular}{|c|c|c|c|c|}
\hline 9 & $<1.5$ & $<.2$ & 1 & $1_{<.7}$ \\
\hline 6 & 25.8 & 15.1 & 20.7 & 21.8 \\
\hline 9 & 27 & 16 & 22 & 21 \\
\hline 9 & 20,200 & 17,100 & 17,100 & 18,100 \\
\hline 9 & 20 & $<13$ & ${ }^{1} 12$ & ${ }^{1} 11$ \\
\hline 9 & 672 & 298 & 500 & 497 \\
\hline 6 & 14.3 & 11.7 & 13.0 & 13.0 \\
\hline 9 & .7 & $<.3$ & $! .4$ & ${ }^{1}<.5$ \\
\hline 9 & 73 & 54 & 63 & 63 \\
\hline
\end{tabular}


Table 22. Statistical summary of fine-grained bed-sediment data for the upper Clark Fork basin, Montana, August 1986 through August 1999 (Continued)

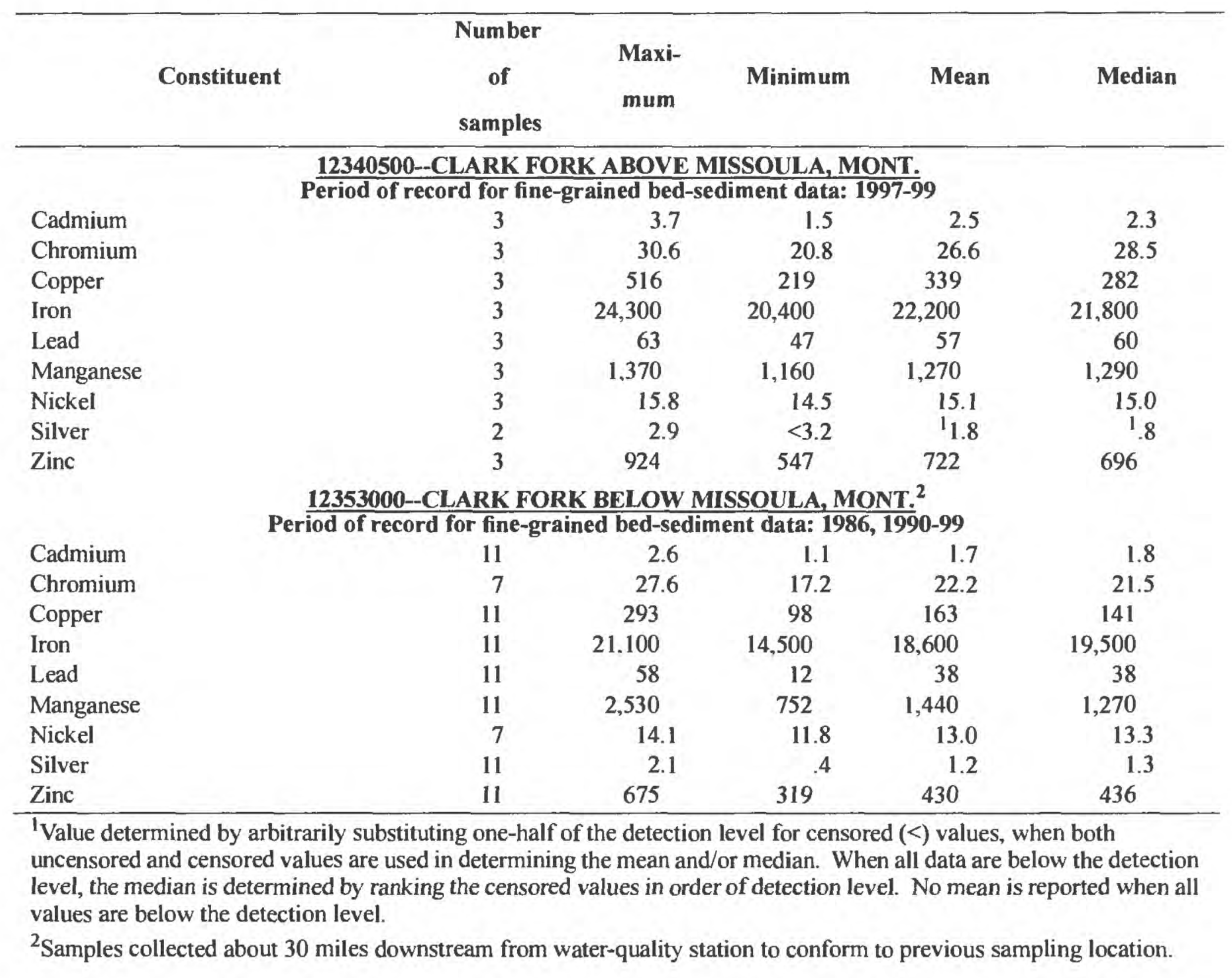


Table 23. Statistical summary of bulk bed-sediment data for the upper Clark Fork basin, Montana, August 1993 through August 1999

[Bulk bed sediment is material smaller than about $10 \mathrm{~mm}$ in diameter. Reported concentrations are in micrograms per gram dry weight. Symbols: <, less than minimum reporting level; --, indicates insufficient data greater than the minimum reporting level to compute statistic. Number of samples represents the number of years that the constituent was analyzed, with each year represented by a single mean concentration of composite samples]

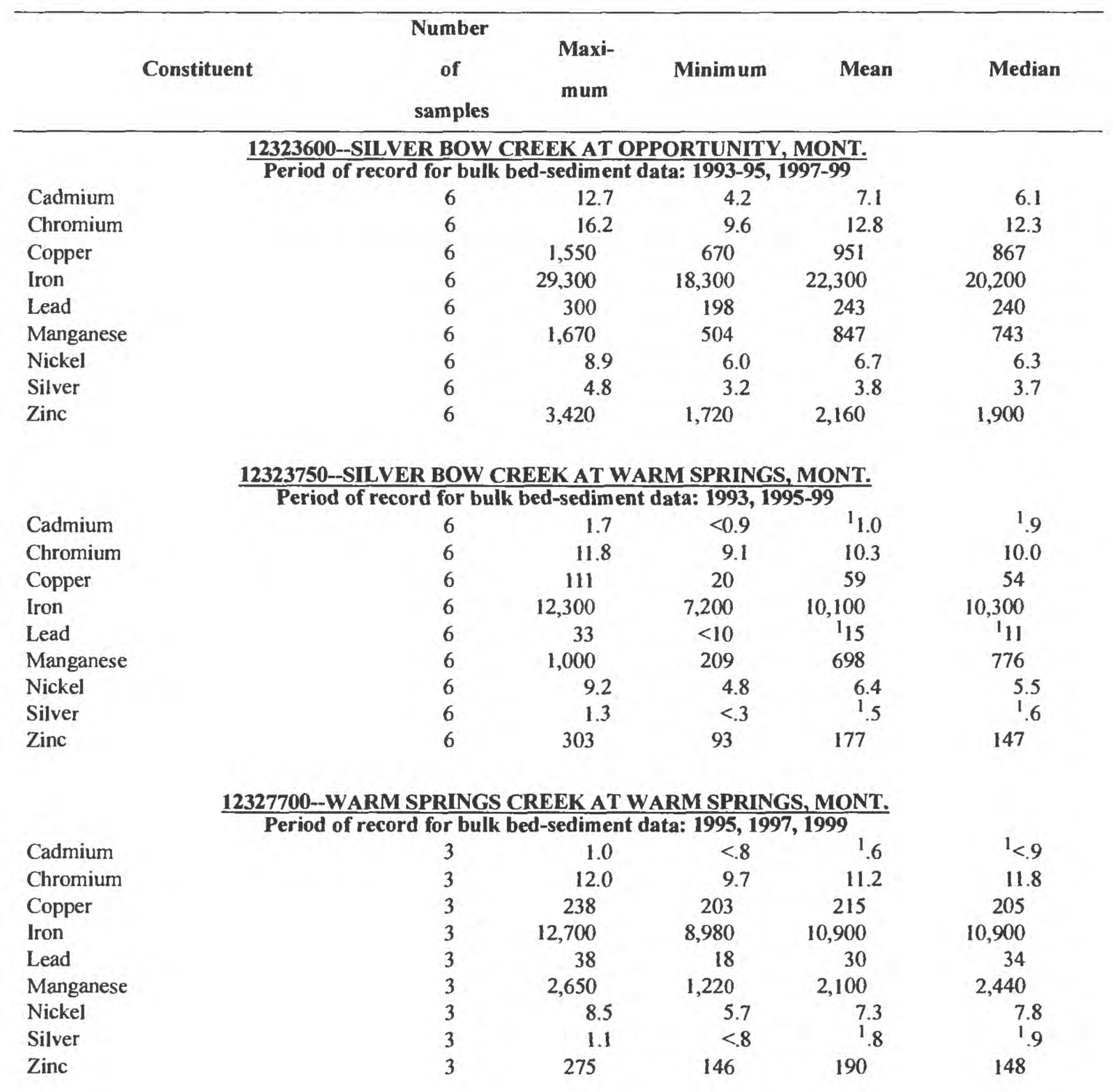


Table 23. Statistical summary of bulk bed-sediment data for the upper Clark Fork basin, Montana, August 1993 through August 1999 (Continued)

\begin{tabular}{|c|c|c|c|c|c|}
\hline Constituent & $\begin{array}{c}\text { Number } \\
\text { of } \\
\text { samples }\end{array}$ & $\begin{array}{l}\text { Maxi- } \\
\text { mum }\end{array}$ & Minimum & Mean & Median \\
\hline \multicolumn{6}{|c|}{$\begin{array}{l}\text { 12323800-CLARK FORK NEAR GALEN, MONT. } \\
\text { Period of record for bulk bed-sediment data: } 1993-99\end{array}$} \\
\hline Cadmium & 7 & 6.0 & $<.9$ & $I_{3,0}$ & ${ }^{1} 2.6$ \\
\hline Chromium & 7 & 23.0 & 4.2 & 13.6 & 14.8 \\
\hline Copper & 7 & 685 & 223 & 392 & 318 \\
\hline Iron & 7 & 31,300 & 9,930 & 20,400 & 20,600 \\
\hline Lead & 7 & 158 & 41 & 76 & 64 \\
\hline Manganese & 7 & 5,410 & 900 & 2,000 & 1,540 \\
\hline Nickel & 7 & 12.5 & 4.9 & 7.7 & 6.9 \\
\hline Silver & 7 & 1.9 & .7 & ${ }^{1} 1.3$ & ${ }^{1} 1.6$ \\
\hline Zinc & 7 & 1,280 & 417 & 671 & 653 \\
\hline \multicolumn{6}{|c|}{$\frac{\text { 461415112450801--CLARK FORK BELOW LOST CREEK, NEAR GALEN, MONT }}{\text { Period of record for bulk bed-sediment data: 1996-99 }}$} \\
\hline Cadmium & 4 & 3.1 & $<.9$ & ${ }^{\prime} 2.0$ & ${ }^{1} 2.2$ \\
\hline Chromium & 4 & 17.5 & 9.3 & 13.0 & 12.5 \\
\hline Copper & 4 & 763 & 238 & 463 & 426 \\
\hline Iron & 4 & 21,000 & 12,600 & 16,700 & 16.600 \\
\hline Lead & 4 & 104 & 41 & 74 & 75 \\
\hline Manganese & 4 & 1,740 & 1,260 & 1,420 & 1,340 \\
\hline Nickel & 4 & 8.2 & 4.2 & 6.7 & 7.2 \\
\hline Silver & 4 & 2.8 & $<3.4$ & ${ }^{1} 1.9$ & ${ }^{1} 1.9$ \\
\hline Zinc & 4 & 787 & 365 & 577 & 577 \\
\hline \multicolumn{6}{|c|}{$\frac{\text { 461559112443301-CLARK FORK NEAR RACETRACK, MONT. }}{\text { Period of record for bulk bed-sediment data: } 1996-99}$} \\
\hline Cadmium & 4 & 3.4 & $<.9$ & ${ }^{1} 2.0$ & ${ }^{1} 2.0$ \\
\hline Chromium & 4 & 16.4 & 12.4 & 14.0 & 13.7 \\
\hline Copper & 4 & 594 & 361 & 442 & 407 \\
\hline Iron & 4 & 19,700 & 16,200 & 18,000 & 18,000 \\
\hline Lead & 4 & 87 & 66 & 77 & 77 \\
\hline Manganese & 4 & 1.680 & 759 & 1,300 & 1,390 \\
\hline Nickel & 4 & 9.9 & 5.5 & 7.3 & 6.9 \\
\hline Silver & 4 & 2.6 & $<3.2$ & ${ }^{11.9}$ & ${ }^{1} 1.7$ \\
\hline Zinc & 4 & 743 & 472 & 594 & 581 \\
\hline \multicolumn{6}{|c|}{$\frac{\text { 461903112440701-CLARK FORK AT DEMPSEY CREEK DIVERSION, NEAR RACETRACK, MONT. }}{\text { Period of record for bulk bed-sediment data: } 1996-99}$} \\
\hline Cadmium & 4 & 3.9 & 1.5 & 2.5 & 2.4 \\
\hline Chromium & 4 & 20.9 & 13.0 & 16.9 & 16.8 \\
\hline Copper & 4 & 651 & 244 & 474 & 501 \\
\hline Iron & 4 & 25,400 & 16,400 & 20,700 & 20,500 \\
\hline Lead & 4 & 89 & 47 & 70 & 73 \\
\hline Manganese & 4 & 1,940 & 825 & 1,380 & 1,360 \\
\hline Nickel & 4 & 12.8 & 5.5 & 8.7 & 8.2 \\
\hline Silver & 4 & 2.8 & $<.8$ & ${ }^{1} 1.7$ & ${ }^{1} 1.8$ \\
\hline Zinc & 4 & 804 & 368 & 556 & 526 \\
\hline
\end{tabular}


Table 23. Statistical summary of bulk bed-sediment data for the upper Clark Fork basin, Montana, August 1993 through August 1999 (Continued)

\begin{tabular}{|c|c|c|c|c|c|}
\hline Constituent & $\begin{array}{c}\text { Number } \\
\text { of } \\
\text { samples }\end{array}$ & $\begin{array}{l}\text { Maxi- } \\
\text { mum }\end{array}$ & Minimum & Mean & Median \\
\hline \multicolumn{6}{|c|}{$\frac{12324200-\text {-CLARK FORK AT DEER LODGE, MONT. }}{\text { Period of record for bulk bed-sediment data: } 1993-99}$} \\
\hline Cadmium & 7 & 3.1 & 1.0 & 2.2 & 2.1 \\
\hline Chromium & 7 & 24.5 & 12.1 & 17.4 & 16.1 \\
\hline Copper & 7 & 691 & 281 & 424 & 383 \\
\hline Iron & 7 & 25,000 & 13,200 & 18,700 & 17,900 \\
\hline Lead & 7 & 85 & 45 & 71 & 74 \\
\hline Manganese & 7 & 2,060 & 653 & 1,090 & 1,020 \\
\hline Nickel & 7 & 12.3 & 7.7 & 9.6 & 10.1 \\
\hline Silver & 7 & 2.8 & $<.7$ & ${ }^{1} 1.5$ & ${ }^{1} 1.6$ \\
\hline Zinc & 7 & 777 & 456 & 565 & 529 \\
\hline \multicolumn{6}{|c|}{$\frac{12324590 \text {-LITTLE BLACKFOOT RIVER NEAR GARRISON, MONT. }}{\text { Period of record for bulk bed-sediment data: } 1994,1998}$} \\
\hline Cadmium & 2 & .7 & $<1.2$ & ${ }^{1} .6$ & - \\
\hline Chromium & 2 & 33.2 & 14.7 & 23.9 & - \\
\hline Copper & 2 & 20 & 19 & 20 & - \\
\hline Iron & 2 & 21,000 & 15,600 & 18,300 & - \\
\hline Lead & 2 & 18 & 12 & 15 & - \\
\hline Manganese & 2 & 420 & 308 & 364 & -- \\
\hline Nickel & 2 & 15.2 & 8.6 & 11.9 & - \\
\hline Silver & 2 & $<1.6$ & $<.7$ & 1 & - \\
\hline Zinc & 2 & 86 & 73 & 79 & - \\
\hline \multicolumn{6}{|c|}{$\frac{\text { 12324680-CLARK FORK AT GOLDCREEK, MONT. }}{\text { Period of record for bulk bed-sediment data: } 1993-99}$} \\
\hline Cadmium & 7 & 5.2 & 1.1 & 3.0 & 2.4 \\
\hline Chromium & 7 & 33.2 & 17.6 & 23.3 & 21.1 \\
\hline Copper & 7 & 858 & 243 & 464 & 370 \\
\hline Iron & 7 & 24,900 & 15,500 & 19,400 & 18,600 \\
\hline Lead & 7 & 86 & 46 & 65 & 71 \\
\hline Manganese & 7 & 2,600 & 554 & 1,220 & 1,191 \\
\hline Nickel & 7 & 15.9 & 9.1 & 12.0 & 12.0 \\
\hline Silver & 7 & 3.7 & $<7$ & ${ }^{1} 1.8$ & ${ }^{1} 1.6$ \\
\hline Zinc & 7 & 1,020 & 525 & 717 & 676 \\
\hline \multicolumn{6}{|c|}{$\frac{12331500-\text {-FLINT CREEK NEAR DRUMMOND, MONT. }}{\text { Period of record for bulk bed-sediment data: } 1993-99}$} \\
\hline Cadmium & 7 & 3.2 & $<.2$ & ${ }^{1} 1.2$ & 1.9 \\
\hline Chromium & 7 & 13.9 & 4.9 & 9.8 & 10.7 \\
\hline Copper & 7 & 40 & 18 & 27 & 25 \\
\hline Iron & 7 & 15,700 & 8,630 & 12,900 & 13,400 \\
\hline Lead & 7 & 120 & 51 & 82 & 80 \\
\hline Manganese & 7 & 3.200 & 1,150 & 2,230 & 2,240 \\
\hline Nickel & 7 & 8.0 & 4.5 & 6.0 & 5.9 \\
\hline Silver & 7 & 5.8 & 3.3 & 4.4 & 4.1 \\
\hline Zinc & 7 & 429 & 190 & 302 & 325 \\
\hline
\end{tabular}


Table 23. Statistical summary of bulk bed-sediment data for the upper Clark Fork basin, Montana, August 1993 through August 1999 (Continued)

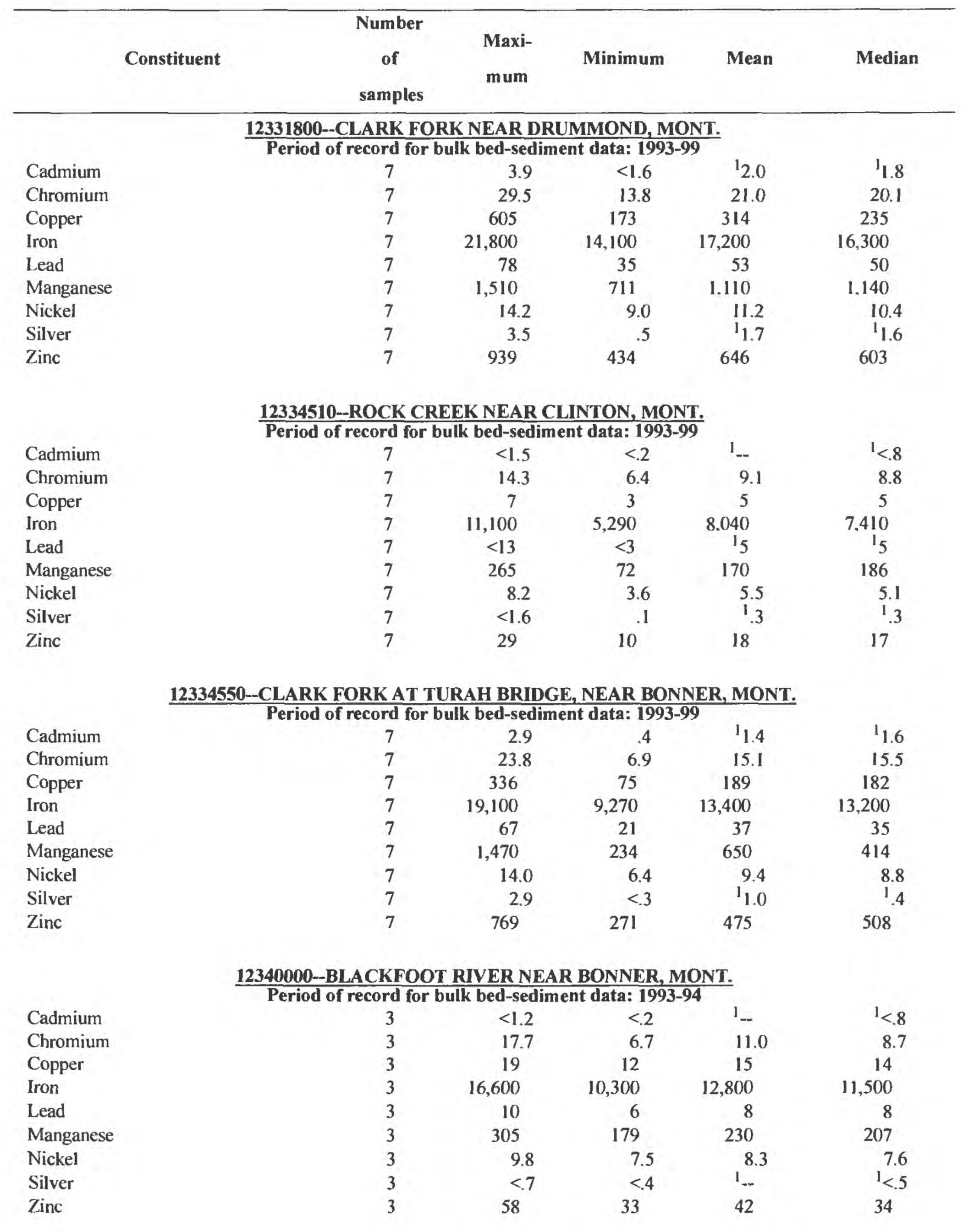


Table 23. Statistical summary of bulk bed-sediment data for the upper Clark Fork basin, Montana, August 1993 through August 1999 (Continued)

\begin{tabular}{|c|c|c|c|c|c|}
\hline Constituent & $\begin{array}{l}\text { Number } \\
\text { of } \\
\text { samples }\end{array}$ & $\begin{array}{l}\text { Maxi- } \\
\text { mum }\end{array}$ & Minimum & Mean & Median \\
\hline \multicolumn{6}{|c|}{$\frac{12340500-C L A R K \text { FORK ABOVE MISSOULA, MONT. }}{\text { Period of record for bulk bed-sediment data: } 1997-99}$} \\
\hline Cadmium & 3 & $<1.6$ & .4 & ${ }^{1} .5$ & $<.8$ \\
\hline Chromium & 3 & 18.2 & 9.7 & 12.7 & 10.2 \\
\hline Copper & 3 & 129 & 43 & 81 & 72 \\
\hline Iron & 3 & 16,000 & 11,500 & 13,800 & 13,800 \\
\hline Lead & 3 & 30 & 7 & 18 & 18 \\
\hline Manganese & 3 & 810 & 228 & 530 & 553 \\
\hline Nickel & 3 & 10.3 & 8.2 & 9.2 & 9.2 \\
\hline Silver & 3 & $<3.3$ & $<.4$ & ${ }^{1} .8$ & ${ }^{1} .6$ \\
\hline Zinc & 3 & 387 & 145 & 265 & 263 \\
\hline \multicolumn{6}{|c|}{$\frac{12353000-\text {-CLARK FORK BELOW MISSOULA, MONT. }}{\text { Period of record for bulk bed-sediment data: } 1993-99}$} \\
\hline Cadmium & 7 & $<1.5$ & $<.2$ & ${ }^{1} .5$ & ${ }^{1}<.5$ \\
\hline Chromium & 7 & 12.6 & 4.4 & 7.4 & 6.8 \\
\hline Copper & 7 & 77 & 16 & 40 & 30 \\
\hline Iron & 7 & 11.300 & 5,830 & 8,230 & 8,420 \\
\hline Lead & 7 & 19 & $<10$ & ${ }^{1} 8$ & 16 \\
\hline Manganese & 7 & 444 & 150 & 332 & 368 \\
\hline Nickel & 7 & 7.1 & 3.5 & 5.1 & 4.5 \\
\hline Silver & 7 & $<1.6$ & $<3$ & ${ }^{1} .4$ & ${ }^{1} .4$ \\
\hline Zinc & 7 & 172 & 58 & 112 & 101 \\
\hline
\end{tabular}

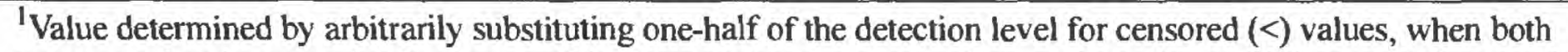
uncensored and censored values are used in determining the mean and/or median. When all data are below the detection level, the median is determined by ranking the censored values in order of detection level. No mean is reported when all values are below the detection level.

${ }^{2}$ Samples collected about 30 miles downstream from water-quality station to conform to previous sampling location. 
Table 24. Statistical summary of biological data for the upper Clark Fork basin, Montana, August 1986 through August 1999

[Concentrations are in micrograms per gram dry weight. Symbols: <, less than minimum reporting level; --, indicates either too few samples (less than three) or insufficient data greater than the minimum reporting level to compute statistic, or element not analyzed, Number of composite samples represents the total of all individual composite samples collected for every year that the constituent was analyzed. Values for single samples are arbitrarily listed in the "Mean" column Because Hydropsyche insects were not sorted to the species level during 1986-89, statistics for stations sampled during those years are based on the results of all Hydropsyche species combined. At some sites, statistics for the Hydropsyche morosa group are based on the combined results for two or more species]

\begin{tabular}{|c|c|c|c|c|c|}
\hline & $\begin{array}{c}\text { Number of } \\
\text { composite } \\
\text { samples }\end{array}$ & Maximum & Minimum & Mean & Median \\
\hline \multicolumn{6}{|c|}{$\frac{12323600-\text { SILVER BOW CREEK AT OPPORTUNITY, MONT. }}{\text { Period of record for biological data: } 1992,1994-95,1997-99}$} \\
\hline \multicolumn{6}{|c|}{ Brachycentrus spp. } \\
\hline Cadmium & 3 & 12.5 & 5.8 & 10.0 & 11.6 \\
\hline Chromium & 3 & 1.1 & .7 & .8 & .7 \\
\hline Copper & 3 & 846 & 587 & 675 & 592 \\
\hline Iron & 3 & 730 & 335 & 476 & 363 \\
\hline Lead & 3 & 17.9 & 7.4 & 11.1 & 7.9 \\
\hline Manganese & 3 & 503 & 231 & 364 & 357 \\
\hline Nickel & 3 & 1.9 & $<.1$ & 1.0 & 1.0 \\
\hline Zinc & 3 & 888 & 629 & 735 & 687 \\
\hline \multicolumn{6}{|c|}{ Hydropsyche cockerelli } \\
\hline Cadmium & 5 & 6.3 & 4.1 & 4.9 & 4.7 \\
\hline Chromium & 5 & 8.0 & 1.0 & 3.7 & 3.1 \\
\hline Copper & 5 & 462 & 269 & 365 & 333 \\
\hline Iron & 5 & 1,180 & 689 & 931 & 953 \\
\hline Lead & 5 & 21.7 & 19.0 & 20.3 & 20.1 \\
\hline Manganese & 5 & 718 & 180 & 460 & 434 \\
\hline Nickel & 5 & 2.1 & .7 & 1.4 & 1.6 \\
\hline Zinc & 5 & 898 & 749 & 818 & 805 \\
\hline \multicolumn{6}{|c|}{ Hvdropsyche tana } \\
\hline Cadmium & 6 & 9.2 & 4.8 & 6.8 & 6.9 \\
\hline Chromium & 6 & 11.5 & .9 & 4.5 & 1.8 \\
\hline Copper & 6 & 456 & 10.5 & 236 & 298 \\
\hline Iron & 6 & 1,520 & 857 & 1.100 & 1.050 \\
\hline Lead & 6 & 21.0 & 15.6 & 18.6 & 18.3 \\
\hline Manganese & 6 & 969 & 307 & 634 & 675 \\
\hline Nickel & 6 & 1.8 & .7 & 1.4 & 1.6 \\
\hline Zinc & 6 & 1,070 & 760 & 961 & 1,020 \\
\hline \multicolumn{6}{|c|}{$\frac{\text { 12323750--SILVER BOW CREEK AT WARM SPRINGS, MONT. }}{\text { Period of record for biological data: } 1992-99}$} \\
\hline \multicolumn{6}{|c|}{ Hydropsyche cockerelli } \\
\hline Cadmium & 21 & 2.1 & .2 & .8 & .6 \\
\hline Chromium & 21 & 1.3 & .5 & .8 & .8 \\
\hline Copper & 21 & 96.9 & 22.4 & 45.4 & 42.5 \\
\hline Iron & 21 & 1,240 & 351 & 691 & 732 \\
\hline Lead & 21 & 5.7 & .3 & 3.3 & 3.0 \\
\hline Manganese & 21 & 2,450 & 491 & 981 & 828 \\
\hline Nickel & 21 & 1.8 & .3 & .8 & .8 \\
\hline Zinc & 21 & 276 & 115 & 184 & 174 \\
\hline
\end{tabular}


Table 24. Statistical summary of biological data for the upper Clark Fork basin, Montana, August 1986 through August 1999 (Continued)

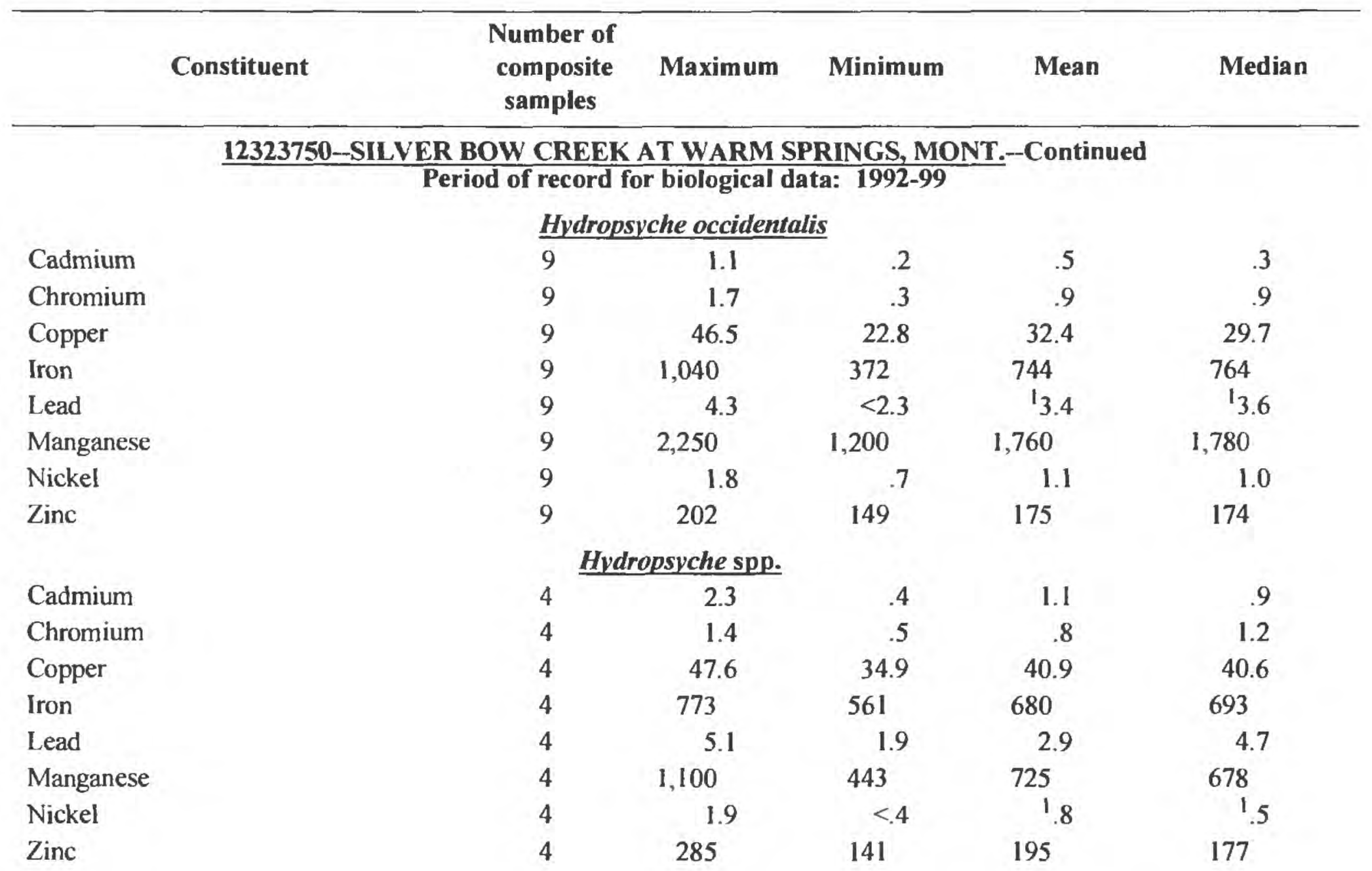

12323770--WARM SPRINGS CREEK AT WARM SPRINGS, MONT. Period of record for biological data: 1995, 1997, 1999

Cadmium

Chromium

Copper

Iron

Lead

Manganese

Nickel

Zinc

Cadmium

Chromium

Copper

Iron

Lead

Manganese

Nickel

Zinc

Cadmium

Chromium

Copper

Iron

Lead

Manganese

Nickel

Zinc

\begin{tabular}{ccccc}
\multicolumn{2}{c}{ Arctopspche grandis } & & & \\
3 & 2.4 & 1.9 & 2.2 & 2.1 \\
3 & 2.9 & 1.4 & 2.0 & 1.8 \\
3 & 102 & 95.6 & 98.9 & 98.8 \\
3 & 1,040 & 684 & 866 & 872 \\
3 & 5.6 & $<6.3$ & $1^{2} 4.4$ & $<8.7$ \\
3 & 2,280 & 1,340 & 1,810 & 1,800 \\
3 & $<7.0$ & 1.8 & $1_{2.5}$ & 2.3 \\
3 & 222 & 180 & 200 & 197
\end{tabular}

Hydropsyche occidentalis

\begin{tabular}{lcccc}
\hline 2 & .8 & .7 & .8 & -- \\
2 & 3.2 & 3.2 & 3.2 & -- \\
2 & 183 & 181 & 182 & -- \\
2 & 2,070 & 1,950 & 2,010 & -- \\
2 & 8.2 & 6.7 & 7.4 & -- \\
2 & 2,480 & 2,400 & 2,440 & - \\
2 & 3.3 & 3.0 & 3.2 & -- \\
2 & 172 & 166 & 169 & -- \\
& Hydropsyche spp. & & & \\
1 & -- & -- & $<9.3$ & -- \\
1 & -- & -- & 1.6 & -- \\
1 & - & -- & 94.8 & -- \\
1 & -- & -- & 1,150 & -- \\
1 & -- & -- & $<16.7$ & -- \\
1 & -- & -- & 956 & -- \\
1 & -- & -- & 2.0 & - \\
1 & -- & - & 129 & -
\end{tabular}


Table 24. Statistical summary of biological data for the upper Clark Fork basin, Montana. August 1986 through August 1999 (Continued)

\begin{tabular}{|c|c|c|c|c|c|}
\hline Constituent & $\begin{array}{c}\text { Number of } \\
\text { composite } \\
\text { samples }\end{array}$ & Maximum & Minimum & Mean & Median \\
\hline \multicolumn{6}{|c|}{$\frac{12323800 \text {-CLARK FORK NEAR GALEN, MONT. }}{\text { Period of record for biological data: } 1987,1991-99}$} \\
\hline \multicolumn{6}{|c|}{ Hydropsyche cockerelli } \\
\hline Cadmium & 14 & 2.7 & 1.3 & 1.7 & 1.6 \\
\hline Chromium & 14 & 3.3 & .8 & 1.6 & 1.5 \\
\hline Copper & 14 & 181 & 48.7 & 92.9 & 91.9 \\
\hline Iron & 14 & 1,510 & 816 & 1,170 & 1,160 \\
\hline Lead & 14 & 11.0 & 1.2 & 7.0 & 7.7 \\
\hline Manganese & 14 & 2,950 & 1,070 & 1,920 & 1,840 \\
\hline Nickel & 14 & 3.1 & 1.0 & 1.6 & 1.3 \\
\hline Zinc & 14 & 299 & 136 & 209 & 210 \\
\hline \multicolumn{6}{|c|}{ Hydropsyche morosa group } \\
\hline Cadmium & 5 & 3.2 & 2.4 & 2.5 & 2.4 \\
\hline Chromium & 5 & 4.6 & 1.8 & 2.6 & 2.2 \\
\hline Copper & 5 & 185 & 156 & 173 & 175 \\
\hline Iron & 5 & 1,890 & 1,360 & 1,510 & 1.430 \\
\hline Lead & 5 & 12.4 & 7.1 & 8.5 & 7.9 \\
\hline Manganese & 5 & 3,960 & 2,360 & 3,500 & 3,860 \\
\hline Nickel & 5 & 3.6 & 1.9 & 2.3 & 2.1 \\
\hline Zinc & 5 & 349 & 292 & 309 & 303 \\
\hline \multicolumn{6}{|c|}{ Hydropsyche occidentalis } \\
\hline Cadmium & 28 & 1.7 & 6 & 1.1 & 1.0 \\
\hline Chromium & 28 & 6.6 & .7 & 1.7 & 1.4 \\
\hline Copper & 28 & 106 & 49.2 & 77.5 & 74.5 \\
\hline Iron & 28 & 1,920 & 642 & 1,180 & 1,140 \\
\hline Lead & 28 & 13.5 & 1.6 & 6.5 & 6.2 \\
\hline Manganese & 28 & 4,070 & 1,220 & 2,340 & 2,170 \\
\hline Nickel & 28 & 3.5 & .8 & 1.5 & 1.4 \\
\hline Zinc & 28 & 278 & 170 & 197 & 193 \\
\hline \multicolumn{6}{|c|}{ Hydropsyche tana } \\
\hline Cadmium & 1 & -- & -- & 1.5 & - \\
\hline Chromium & 1 & - & - & 1.4 & - \\
\hline Copper & 1 & - & -- & 92.9 & -- \\
\hline Iron & 1 & - & -- & 1,340 & - \\
\hline Lead & 1 & - & - & 9.0 & - \\
\hline Manganese & 1 & - & -- & 2,160 & -- \\
\hline Nickel & 1 & - & -- & 2.1 & -- \\
\hline Zinc & 1 & -- & - & 206 & -- \\
\hline \multicolumn{6}{|c|}{ Hydropsvche spp. } \\
\hline Cadmium & 4 & 3.5 & 2.6 & 3.0 & 3.0 \\
\hline Chromium & 0 & -- & - & -- & - \\
\hline Copper & 4 & 154 & 135 & 148 & 152 \\
\hline Iron & 4 & 1,540 & 1.190 & 1,400 & 1,450 \\
\hline Lead & 4 & 135 & 10.5 & 12.2 & 12.4 \\
\hline Manganese & 0 & - & - & -- & - \\
\hline Nickel & 0 & -- & - & - & - \\
\hline Zinc & 4 & 329 & 279 & 308 & 313 \\
\hline
\end{tabular}

88 Water-quality, bed-sediment, and biological data (October 1998 through September 1999) and statistical summaries of data for streams in the Upper Clark Fork Basin, Montana 
Table 24. Statistical summary of biological data for the upper Clark Fork basin, Montana, August 1986 through August 1999 (Continued)

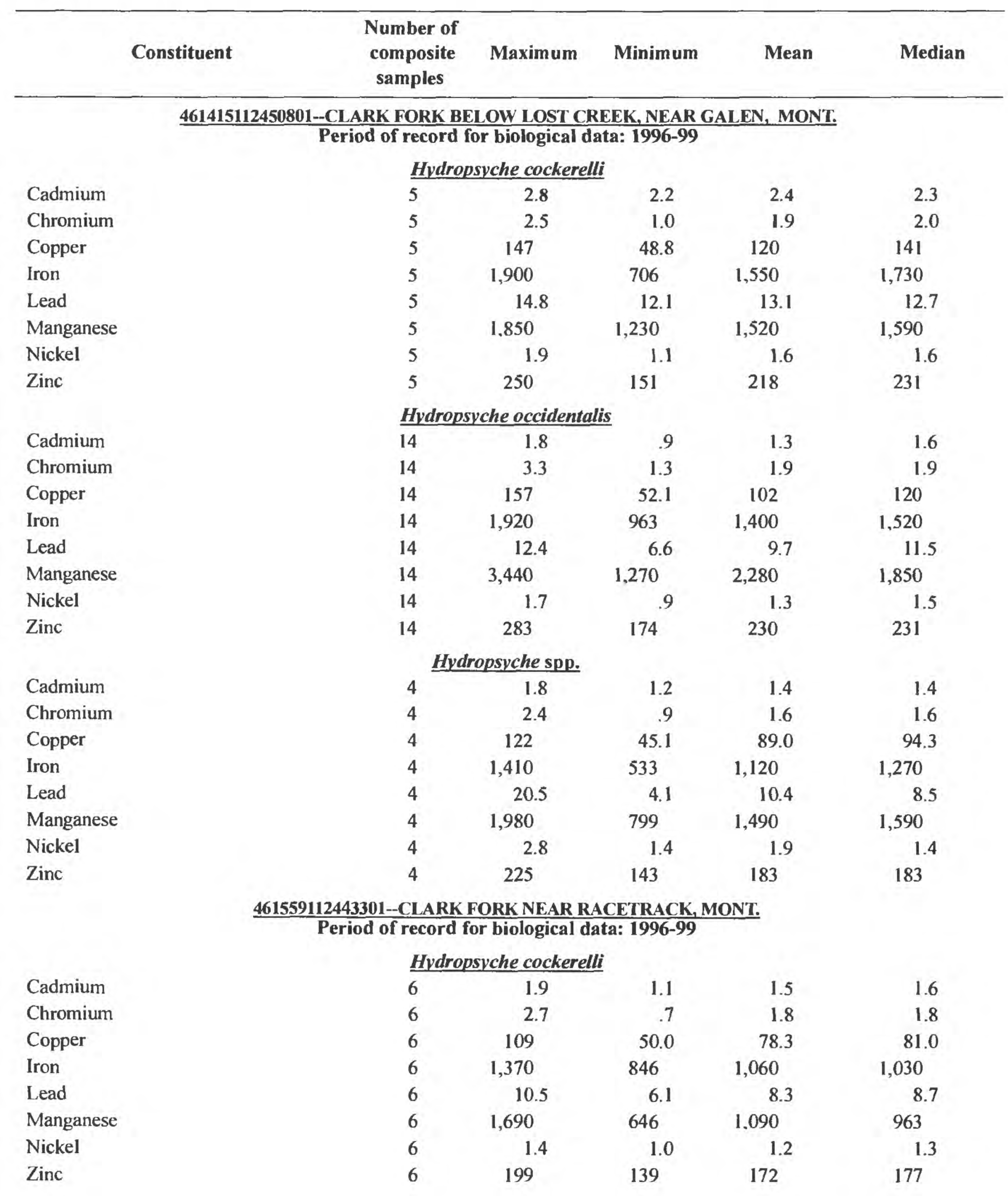


Table 24. Statistical summary of biological data for the upper Clark Fork basin, Montana, August 1986 through August 1999 (Continued)

\begin{tabular}{|c|c|c|c|c|c|}
\hline Constituent & $\begin{array}{l}\text { Number of } \\
\text { composite } \\
\text { samples }\end{array}$ & Maximum & Minimum & Mean & Median \\
\hline \multicolumn{6}{|c|}{$\frac{\text { 461559112443301--CLARK FORK NEAR RACETRACK, MONT. -_Continued }}{\text { Period of record for biological data: } 1996-99}$} \\
\hline \multicolumn{6}{|c|}{ Hydropsyche occidentalis } \\
\hline Cadmium & 11 & 2.2 & .7 & 1.3 & 1.4 \\
\hline Chromium & 11 & 2.6 & 1.1 & 1.9 & 2.0 \\
\hline Copper & 11 & 160 & 59.5 & 103 & 93.5 \\
\hline Iron & 11 & 1,880 & $\mathrm{I}, 030$ & 1,470 & 1,450 \\
\hline Lead & 11 & 11.7 & 4.3 & 9.6 & 9.7 \\
\hline Manganese & 11 & 3,480 & 1,090 & 2,030 & 2,100 \\
\hline Nickel & 11 & 1.7 & 1.1 & 1.3 & 1.2 \\
\hline Zinc & 11 & 255 & 181 & 225 & 229 \\
\hline \multicolumn{6}{|c|}{ Hydropsyche spp. } \\
\hline Cadmium & I & -- & -- & 1.0 & - \\
\hline Chromium & I & -- & - & .7 & -- \\
\hline Copper & 1 & - & - & 82.9 & -- \\
\hline Iron & 1 & - & - & 1,140 & - \\
\hline Lead & 1 & -- & -- & 5.7 & -- \\
\hline Manganese & 1 & -- & - & 910 & - \\
\hline Nickel & 1 & -- & -- & 1.1 & -- \\
\hline Zinc & 1 & - & - & 151 & -- \\
\hline \multicolumn{6}{|c|}{$\frac{\text { 461903112440701--CLARK FORK AT DEMPSEY CREEK DIVERSION, NEAR RACETRACK, MONT. }}{\text { Period of record for biological data: 1996-99 }}$} \\
\hline \multicolumn{6}{|c|}{ Arctopsyche grandis } \\
\hline Cadmium & I & -- & -- & 1.7 & - \\
\hline Chromium & 1 & - & - & $<2.4$ & - \\
\hline Copper & 1 & - & - & 30.8 & - \\
\hline Iron & 1 & -- & - & 340 & - \\
\hline Lead & 1 & -- & -- & $<14.5$ & - \\
\hline Manganese & 1 & -- & -- & 510 & -- \\
\hline Nickel & 1 & - & - & 1.0 & - \\
\hline Zinc & $\mathbf{I}$ & -- & -- & 87 & - \\
\hline \multicolumn{6}{|c|}{ Hydropsyche cockerelli } \\
\hline Cadmium & 2 & 1.6 & .9 & 1.2 & - \\
\hline Chromium & 2 & 1.3 & 1.0 & 1.2 & - \\
\hline Copper & 2 & 143 & 87.6 & 115 & - \\
\hline Iron & 2 & 1,290 & 831 & 1,060 & - \\
\hline Lead & 2 & 8.4 & 6.8 & 7.6 & - \\
\hline Manganese & 2 & 697 & 487 & 592 & - \\
\hline Nickel & 2 & 1.9 & 1.6 & 1.8 & - \\
\hline Zinc & 2 & 180 & 162 & 171 & -- \\
\hline
\end{tabular}


Table 24. Statistical summary of biological data for the upper Clark Fork basin. Montana, August 1986 through August 1999 (Continued)

\begin{tabular}{|c|c|c|c|c|c|}
\hline Constituent & $\begin{array}{l}\text { Number of } \\
\text { composite } \\
\text { samples }\end{array}$ & Maximum & Minimum & Mean & Median \\
\hline \multicolumn{6}{|c|}{$\frac{\text { 461903112440701-CLARK FORK AT DEMPSEY CREEK DIVERSION, NEAR RACETRACK, MONT.-Continued }}{\text { Period of record for biological data: } 1996-99}$} \\
\hline \multicolumn{6}{|c|}{ Hydropsyche occidentalis } \\
\hline Cadmium & 12 & 1.7 & .7 & 1.1 & 1.0 \\
\hline Chromium & 12 & 2.0 & 1.2 & 1.7 & 1.8 \\
\hline Copper & 12 & 163 & 74.9 & 98.1 & 87.1 \\
\hline Iron & 12 & 1,660 & 1,100 & 1,480 & 1,500 \\
\hline Lead & 12 & 13.8 & 9.7 & 11.3 & 11.3 \\
\hline Manganese & 12 & 3,990 & 826 & 2,710 & 2,370 \\
\hline Nickel & 12 & 2.4 & 1.2 & 1.5 & 1.4 \\
\hline Zinc & 12 & 292 & 222 & 250 & 238 \\
\hline \multicolumn{6}{|c|}{ Hydropsyche spp. } \\
\hline Cadmium & 2 & 1.7 & 1.6 & 1.6 & -- \\
\hline Chromium & 2 & 2.1 & 1.4 & 1.8 & -- \\
\hline Copper & 2 & 140 & 104 & 122 & -- \\
\hline Iron & 2 & 1,610 & 1,070 & 1,340 & - \\
\hline Lead & 2 & 13.2 & 10.5 & 11.8 & - \\
\hline Manganese & 2 & 1,150 & 638 & 892 & -- \\
\hline Nickel & 2 & 1.6 & 1.6 & 1.6 & -- \\
\hline Zinc & 2 & 212 & 191 & 202 & -- \\
\hline \multicolumn{6}{|c|}{$\frac{12324200-\text {-CLARK FORK AT DEER LODGE, MONT. }}{\text { Period of record for biological data: } 1986-87,1990-99}$} \\
\hline \multicolumn{6}{|c|}{ Arctopsyche grandis } \\
\hline Cadmium & 2 & 2.4 & $<4.2$ & ${ }^{1} 2.2$ & - \\
\hline Chromium & 2 & 1.0 & $<1.3$ & ${ }^{1} .8$ & -- \\
\hline Copper & 2 & 69.1 & 34.9 & 52.0 & - \\
\hline Iron & 2 & 676 & 537 & 606 & - \\
\hline Lead & 2 & $<7.8$ & 3.8 & $1^{1} 3.8$ & -- \\
\hline Manganese & 2 & 727 & 380 & 554 & -- \\
\hline Nickel & 2 & $<1.7$ & $<1.3$ & $1-$ & -- \\
\hline Zinc & 2 & 178 & 140 & 159 & -- \\
\hline \multicolumn{6}{|c|}{ Hydropsvche cockerelli } \\
\hline Cadmium & 18 & 2.3 & .8 & 1.4 & 1.3 \\
\hline Chromium & 18 & 3.2 & .4 & 1.7 & 1.8 \\
\hline Copper & 18 & 136 & 54.7 & 94.6 & 100 \\
\hline Iron & 18 & 3,340 & 490 & 1,170 & 1,050 \\
\hline Lead & 18 & 18.2 & 4.3 & 9.4 & 8.9 \\
\hline Manganese & 18 & 1,030 & 396 & 689 & 686 \\
\hline Nickel & 18 & 2.4 & 3 & 1.3 & 1.3 \\
\hline Zinc & 18 & 391 & 132 & 183 & 180 \\
\hline
\end{tabular}


Table 24. Statistical summary of biological data for the upper Clark Fork basin, Montana, August 1986 through August 1999 (Continued)

\begin{tabular}{|c|c|c|c|c|c|}
\hline & $\begin{array}{c}\text { Number of } \\
\text { composite } \\
\text { samples }\end{array}$ & Maximum & Minimum & Mean & Median \\
\hline \multicolumn{6}{|c|}{$\frac{12324200 \text {-CLARK FORK AT DEER LODGE, MONT.--Continued }}{\text { Period of record for biological data: } 1986-87,1990-99}$} \\
\hline \multicolumn{6}{|c|}{ Hydropsyche occidentalis } \\
\hline Cadmium & 30 & 2.7 & .8 & 1.3 & 1.3 \\
\hline Chromium & 30 & 2.6 & .6 & 1.9 & 1.9 \\
\hline Copper & 30 & 162 & 49.5 & 111 & 110 \\
\hline Iron & 30 & 1,930 & 558 & 1.420 & 1,460 \\
\hline Lead & 30 & 16.2 & 3.5 & 10.9 & 10.8 \\
\hline Manganese & 30 & 2,840 & 649 & 1,650 & 1,720 \\
\hline Nickel & 30 & 12.9 & 1.0 & 1.9 & 1.5 \\
\hline Zinc & 30 & 299 & 166 & 233 & 227 \\
\hline \multicolumn{6}{|c|}{ Hydropsyche spp. } \\
\hline Cadmium & 3 & 2.0 & 1.2 & 1.6 & 1.6 \\
\hline Chromium & 0 & -- & - & - & - \\
\hline Copper & 3 & 222 & 103 & 145 & 111 \\
\hline Iron & 3 & 2,220 & 1.110 & 1,520 & 1,240 \\
\hline Lead & 3 & 15.0 & 5.6 & 8.8 & 5.7 \\
\hline Manganese & 0 & -- & -- & - & -- \\
\hline Nickel & 0 & - & - & - & -- \\
\hline Zinc & 3 & 203 & 185 & 195 & 197 \\
\hline \multicolumn{6}{|c|}{$\frac{12324590 \text {--LITTLE BLACKFOOT RIVER NEAR GARRISON, MONT. }}{\text { Period of record for biological data: } 1987,1994,1998}$} \\
\hline \multicolumn{6}{|c|}{ Arctopsyche grandis } \\
\hline Cadmium & 10 & .5 & .2 & 3 & .3 \\
\hline Chromium & 10 & 1.6 & .6 & .8 & .8 \\
\hline Copper & 10 & 14.0 & 9.1 & 11.4 & 11.5 \\
\hline Iron & 10 & 654 & 177 & 284 & 235 \\
\hline Lead & 10 & 1.3 & .5 & .8 & .8 \\
\hline Manganese & 10 & 596 & 318 & 479 & 496 \\
\hline Nickel & 10 & .6 & .4 & .5 & .5 \\
\hline Zinc & 10 & 179 & 113 & 149 & 145 \\
\hline \multicolumn{6}{|c|}{ Claassenia sabulosa } \\
\hline Cadmium & 5 & .5 & .1 & .3 & .2 \\
\hline Chromium & 5 & .9 & .7 & .8 & .8 \\
\hline Copper & 5 & 36.1 & 20.0 & 29.5 & 31.4 \\
\hline Iron & 5 & 319 & 98 & 174 & 144 \\
\hline Lead & 5 & $<1.2$ & $<.4$ & 1 & ${ }^{1}<.6$ \\
\hline Manganese & 5 & 71.0 & 46.7 & 57.8 & 56.7 \\
\hline Nickel & 5 & .7 & .5 & .6 & .5 \\
\hline Zinc & 5 & 233 & 191 & 205 & 202 \\
\hline
\end{tabular}


Table 24. Statistical summary of biological data for the upper Clark Fork basin, Montana, August 1986 through August 1999 (Continued)

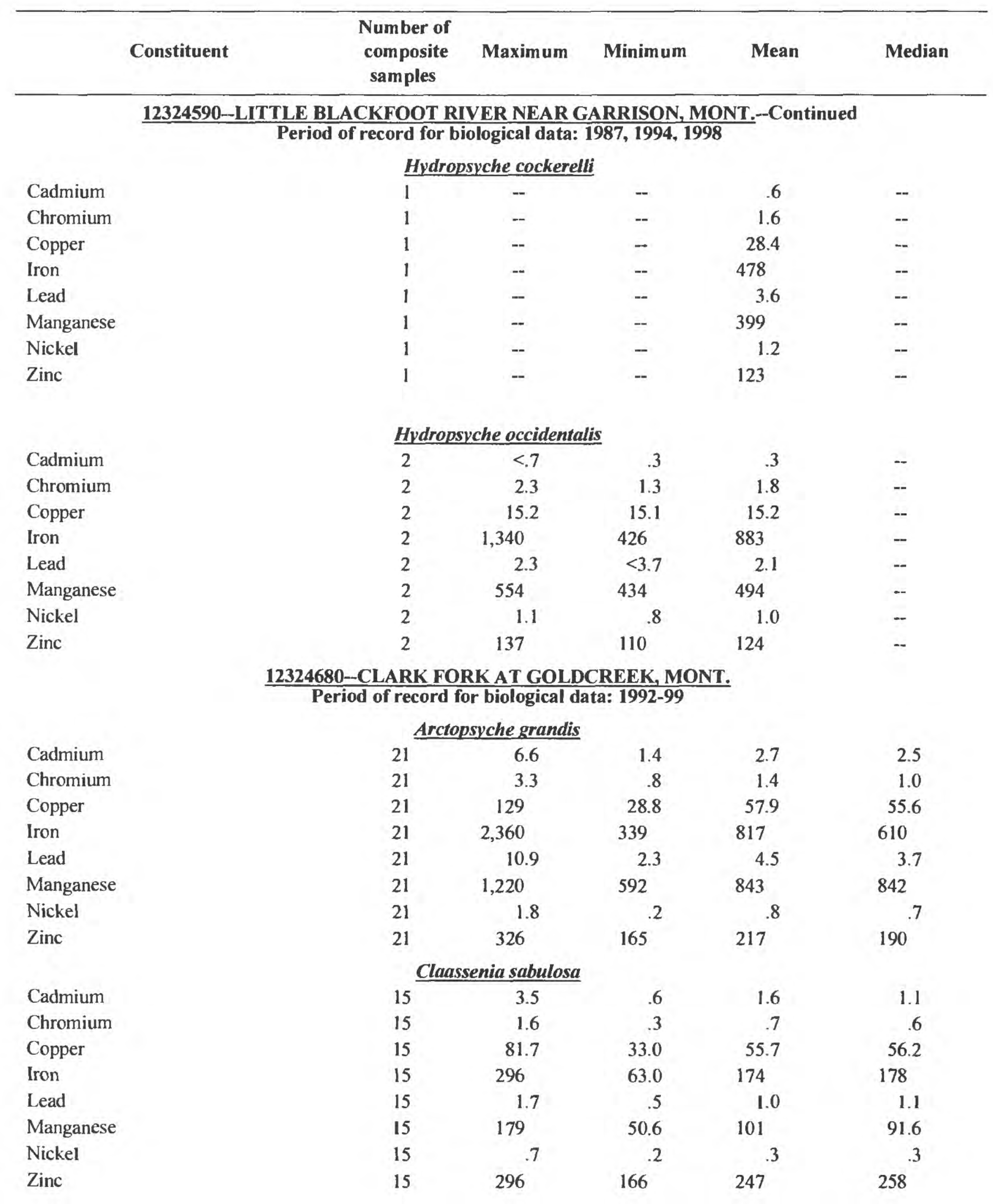


Table 24. Statistical summary of biological data for the upper Clark Fork basin, Montana. August 1986 through August 1999 (Continued)

\begin{tabular}{|c|c|c|c|c|c|}
\hline Constituent & $\begin{array}{c}\text { Number of } \\
\text { composite } \\
\text { samples }\end{array}$ & Maximum & Minimum & Mean & Median \\
\hline \multicolumn{6}{|c|}{$\frac{\text { 12324680--CLARK FORK AT GOLDCREEK, MONT.-Continued }}{\text { Period of record for biological data: } 1992-99}$} \\
\hline \multicolumn{6}{|c|}{ Hydropsyche cockerelli } \\
\hline Cadmium & 14 & 2.6 & .6 & 1.8 & 1.8 \\
\hline Chromium & 14 & 4.7 & .7 & 2.5 & 2.0 \\
\hline Copper & 14 & 188 & 33.5 & 92.8 & 66.9 \\
\hline Iron & 14 & 3,250 & 589 & 1,390 & 1,210 \\
\hline Lead & 14 & 16.2 & 4.5 & 8.3 & 6.5 \\
\hline Manganese & 14 & 954 & 538 & 719 & 670 \\
\hline Nickel & 14 & 2.3 & .6 & 1.4 & 1.3 \\
\hline Zinc & 14 & 240 & 137 & 195 & 204 \\
\hline \multicolumn{6}{|c|}{ Hydropsyche morosa group } \\
\hline Cadmium & 4 & 1.7 & 1.1 & 1.4 & 1.4 \\
\hline Chromium & 4 & 1.4 & 1.3 & 1.4 & 1.4 \\
\hline Copper & 4 & 72.9 & 43.8 & 60.5 & 62.7 \\
\hline Iron & 4 & 1,320 & 612 & 1,050 & 1.130 \\
\hline Lead & 4 & 6.9 & 2.4 & 4.6 & 4.6 \\
\hline Manganese & 4 & 1,030 & 538 & 804 & 822 \\
\hline Nickel & 4 & 1.4 & .9 & 1.2 & 1.2 \\
\hline Zinc & 4 & 190 & 137 & 167 & 170 \\
\hline \multicolumn{6}{|c|}{ Hydropsyche occidentalis } \\
\hline Cadmium & 14 & 1.7 & .7 & 1.3 & 1.4 \\
\hline Chromium & 14 & 3.9 & .4 & 1.5 & 1.5 \\
\hline Copper & 14 & 156 & 26.4 & 64.8 & 58.4 \\
\hline Iron & 14 & 2,720 & 466 & 1,130 & 1,040 \\
\hline Lead & 14 & 15.7 & 2.9 & 6.9 & 6.0 \\
\hline Manganese & 14 & 1,800 & 530 & 1,090 & 1,020 \\
\hline Nickel & 14 & 2.5 & .8 & 1.2 & 1.1 \\
\hline Zinc & 14 & 242 & 97 & 189 & 190 \\
\hline \multicolumn{6}{|c|}{ 12331500-FLINT CREEK NEAR DRUMMOND, MONT. } \\
\hline \multicolumn{6}{|c|}{ Period of record for biological data: $1986,1992-99$} \\
\hline \multicolumn{6}{|c|}{ Arctopsyche grandis } \\
\hline Cadmium & 35 & .8 & .1 & .4 & .4 \\
\hline Chromium & 35 & 4.7 & .6 & 1.9 & 1.8 \\
\hline Copper & 35 & 21.7 & 8.7 & 15.0 & 15.0 \\
\hline Iron & 35 & 2,460 & 606 & 1,370 & 1,330 \\
\hline Lead & 35 & 17.5 & 3.7 & 8.8 & 7.8 \\
\hline Manganese & 35 & 2,480 & 679 & 1,470 & 1,340 \\
\hline Nickel & 35 & 2.7 & .6 & 1.3 & 1.3 \\
\hline Zinc & 35 & 275 & 141 & 198 & 188 \\
\hline
\end{tabular}


Table 24. Statistical summary of biological data for the upper Clark Fork basin, Montana, August 1986 through August 1999 (Continued)

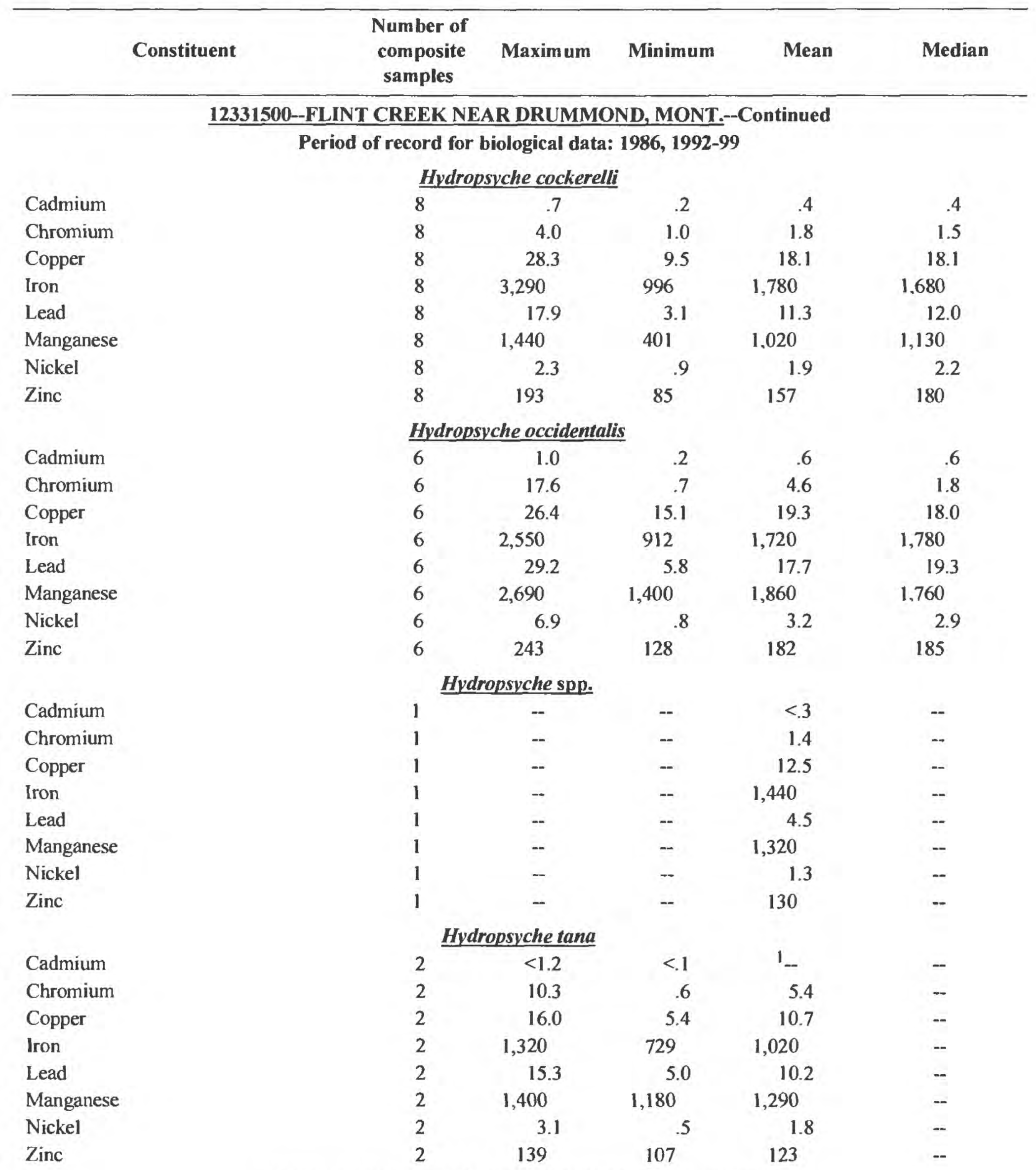

\section{0--CLARK FORK NEAR DRUMMOND, MONT.}

Period of record for biological data: 1986, 1991-99

Cadmium

Chromium

Copper

Iron

Lead

Manganese

Nickel

Zinc

\section{Arctopsvche grandis}

$\begin{array}{lcccc}24 & 3.8 & .7 & 1.7 & 1.5 \\ 24 & 2.5 & .2 & 1.1 & 1.0 \\ 24 & 89.2 & 18.2 & 38.7 & 35.1 \\ 24 & 1,660 & 240 & 666 & 613 \\ 24 & 11.8 & 2.1 & 4.9 & 4.2 \\ 24 & 2,010 & 462 & 884 & 736 \\ 24 & 1.9 & .2 & .7 & .7 \\ 24 & 308 & 142 & 196 & 190\end{array}$


Table 24. Statistical summary of biological data for the upper Clark Fork basin, Montana, August 1986 through August 1999 (Continued)

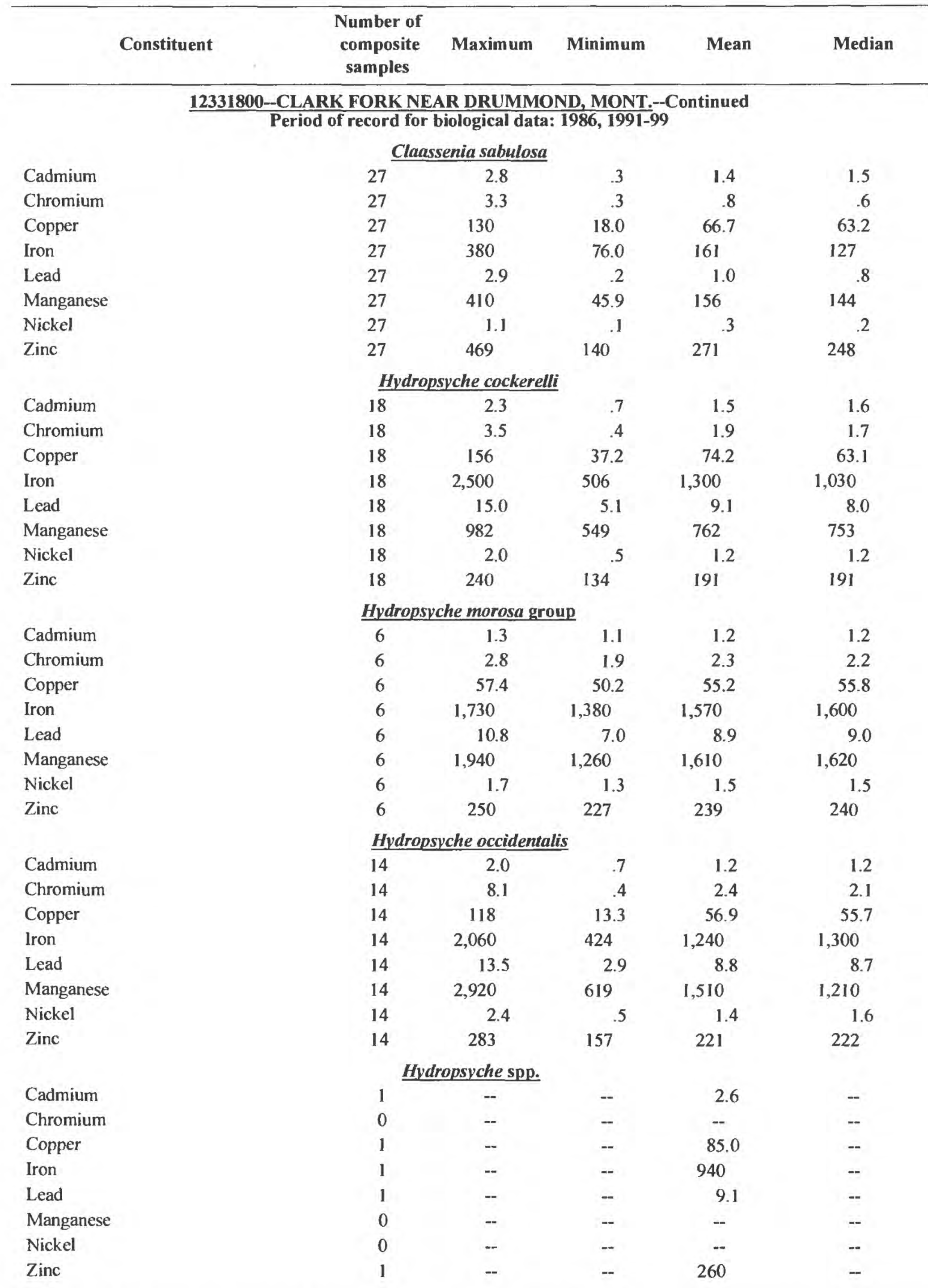

96 Water-quality, bed-sediment, and biological data (October 1998 through September 1999) and statistical summaries of data for streams in the Upper Clark Fork Basin, Montana 
Table 24. Statistical summary of biological data for the upper Clark Fork basin, Montana, August 1986 through August 1999 (Continued)

\begin{tabular}{|c|c|c|c|c|c|}
\hline Constituent & $\begin{array}{c}\text { Number of } \\
\text { composite } \\
\text { samples }\end{array}$ & Maximum & Minimum & Mean & Median \\
\hline \multicolumn{6}{|c|}{$\frac{12334510-\text {-ROCK CREEK NEAR CLINTON, MONT. }}{\text { Period of record for biological data: } 1987,1991-99}$} \\
\hline \multicolumn{6}{|c|}{ Arctopsyche grandis } \\
\hline Cadmium & 32 & .4 & .06 & .2 & .2 \\
\hline Chromium & 32 & 2.9 & .5 & 1.2 & 1.0 \\
\hline Copper & 32 & 15.7 & 4.7 & 8.6 & 8.3 \\
\hline Iron & 32 & 991 & 191 & 517 & 464 \\
\hline Lead & 32 & $<2.9$ & .1 & ${ }^{1} .4$ & ${ }^{1} .4$ \\
\hline Manganese & 32 & 454 & 113 & 249 & 226 \\
\hline Nickel & 32 & 1.6 & .2 & .8 & .9 \\
\hline Zinc & 32 & 189 & 84 & 127 & 124 \\
\hline \multicolumn{6}{|c|}{ Claassenia sabulosa } \\
\hline Cadmium & 16 & .3 & .05 & .2 & .1 \\
\hline Chromium & 16 & 1.8 & .4 & .8 & 6 \\
\hline Copper & 16 & 40.7 & 18.1 & 29.8 & 30.0 \\
\hline Iron & 16 & 129 & 49.8 & 90.7 & 93.1 \\
\hline Lead & 16 & 1.0 & .1 & .3 & .3 \\
\hline Manganese & 16 & 76.3 & 15.7 & 35.0 & 33.6 \\
\hline Nickel & 16 & .9 & .1 & .3 & .3 \\
\hline Zinc & 16 & 264 & 164 & 205 & 207 \\
\hline \multicolumn{6}{|c|}{ Hydropsyche cockerelli } \\
\hline Cadmium & 3 & $<.2$ & $<.2$ & 1.- & $<.2$ \\
\hline Chromium & 3 & 1.0 & .9 & .9 & .9 \\
\hline Copper & 3 & 13.1 & 6.0 & 8.6 & 6.6 \\
\hline Iron & 3 & 609 & 485 & 530 & 497 \\
\hline Lead & 3 & $<1.1$ & $<1.1$ & '- & $<1.1$ \\
\hline Manganese & 3 & 258 & 192 & 219 & 208 \\
\hline Nickel & 3 & .9 & .4 & .6 & .4 \\
\hline Zinc & 3 & 99 & 82 & 89 & 86 \\
\hline \multicolumn{6}{|c|}{ Hydropsyche occidentalis } \\
\hline Cadmium & 4 & $<1.0$ & $<.3$ & 1 & $<.3$ \\
\hline Chromium & 4 & 2.4 & .9 & 1.6 & .9 \\
\hline Copper & 4 & 17.6 & 9.6 & 12.0 & 10.2 \\
\hline Iron & 4 & 752 & 520 & 642 & 648 \\
\hline Lead & 4 & 6.0 & 1.2 & 3.0 & 1.2 \\
\hline Manganese & 4 & 268 & 169 & 228 & 215 \\
\hline Nickel & 4 & 1.7 & .6 & 1.2 & .9 \\
\hline Zinc & 4 & 144 & 99 & 121 & 117 \\
\hline
\end{tabular}


Table 24. Statistical summary of biological data for the upper Clark Fork basin, Montana, August 1986 through August 1999 (Continued)

\begin{tabular}{|c|c|c|c|c|c|}
\hline & $\begin{array}{c}\text { Number of } \\
\text { composite } \\
\text { samples }\end{array}$ & Maximum & Minimum & Mean & Median \\
\hline \multicolumn{6}{|c|}{$\frac{\text { 12334510--ROCK CREEK NEAR CLINTON, MONT--Continued }}{\text { Period of record for biological data: } 1987,1991-99}$} \\
\hline \multicolumn{6}{|c|}{ Hydropsyche spp. } \\
\hline Cadmium & 3 & .3 & $<.5$ & '. 2 & .2 \\
\hline Chromium & 3 & 2.1 & 1.1 & 1.6 & 1.7 \\
\hline Copper & 3 & 16.2 & 11.6 & 14.3 & 15.0 \\
\hline Iron & 3 & 1,140 & 837 & 1,000 & 1,030 \\
\hline Lead & 3 & $<3.1$ & $<1.8$ & 1 & $<2.9$ \\
\hline Manganese & 3 & 462 & 299 & 399 & 437 \\
\hline Nickel & 3 & 1.3 & .8 & 1.1 & 1.1 \\
\hline Zinc & 3 & 135 & 117 & 126 & 126 \\
\hline \multicolumn{6}{|c|}{$\frac{\text { 12334550--CLARK FORK AT TURAH BRIDGE, NEAR BONNER, MONT. }}{\text { Period of record for biological data: } 1986,1991-99}$} \\
\hline \multicolumn{6}{|c|}{ Arctopsyche grandis } \\
\hline Cadmium & 32 & 2.7 & .6 & 1.4 & 1.5 \\
\hline Chromium & 32 & 4.1 & .6 & 1.8 & 1.7 \\
\hline Copper & 32 & 125 & 20.1 & 42.2 & 34.1 \\
\hline Iron & 32 & 2,870 & 420 & 1,060 & 908 \\
\hline Lead & 32 & 13.2 & 2.1 & 4.5 & 3.7 \\
\hline Manganese & 32 & 893 & 351 & 625 & 625 \\
\hline Nickel & 32 & 2.6 & .4 & 1.1 & .9 \\
\hline Zinc & 32 & 276 & 152 & 201 & 197 \\
\hline \multicolumn{6}{|c|}{ Claassenia sabulosa } \\
\hline Cadmium & 23 & 2.5 & .3 & 1.2 & .9 \\
\hline Chromium & 23 & 2.0 & .4 & .7 & .6 \\
\hline Copper & 23 & 79.2 & 38.3 & 56.9 & 54.4 \\
\hline Iron & 23 & 181 & 58.6 & 105 & 102 \\
\hline Lead & 23 & 1.6 & .2 & .6 & .6 \\
\hline Manganese & 23 & 139 & 42.0 & 78.1 & 69.6 \\
\hline Nickel & 23 & .6 & .1 & .2 & .1 \\
\hline Zinc & 23 & 283 & 144 & 221 & 230 \\
\hline \multicolumn{6}{|c|}{ Hydropsyche cockerelli } \\
\hline Cadmium & 21 & 1.7 & .6 & 1.0 & .9 \\
\hline Chromium & 21 & 8.0 & 1.0 & 2.2 & 1.7 \\
\hline Copper & 21 & 118 & 26.4 & 52.0 & 42.5 \\
\hline Iron & 21 & 2,530 & 688 & 1.280 & 1,060 \\
\hline Lead & 21 & 12.1 & 2.2 & 5.4 & 5.0 \\
\hline Manganese & 21 & 788 & 426 & 584 & 560 \\
\hline Nickel & 21 & 26 & .6 & 1.2 & 1.2 \\
\hline Zinc & 21 & 228 & 148 & 186 & 180 \\
\hline
\end{tabular}


Table 24. Statistical summary of biological data for the upper Clark Fork basin. Montana. August 1986 through August 1999 (Continued)

\begin{tabular}{|c|c|c|c|c|c|}
\hline & $\begin{array}{c}\text { Number of } \\
\text { composite } \\
\text { samples }\end{array}$ & Maximum & Minimum & Mean & Median \\
\hline \multicolumn{6}{|c|}{$\frac{\text { 12334550-CLARK FORK AT TURAH BRIDGE, NEAR BONNER, MONT.--Continued }}{\text { Period of record for biological data: } 1986,1991-99}$} \\
\hline \multicolumn{6}{|c|}{ Hydropsyche morosa group } \\
\hline Cadmium & 2 & 1.3 & 1.1 & 1.2 & - \\
\hline Chromium & 2 & 4.6 & 2.4 & 3.5 & -- \\
\hline Copper & 2 & 84.1 & 26.8 & 55.4 & -- \\
\hline Iron & 2 & 1,800 & 986 & 1,390 & -- \\
\hline Lead & 2 & 6.6 & $<7.8$ & 5.2 & -- \\
\hline Manganese & 2 & 1,320 & 537 & 928 & -- \\
\hline Nickel & 2 & 1.7 & 1.3 & 1.5 & -- \\
\hline Zinc & 2 & 231 & 171 & 201 & -- \\
\hline \multicolumn{6}{|c|}{ Hydropsyche occidentalis } \\
\hline Cadmium & 16 & 1.8 & .3 & .9 & .9 \\
\hline Chromium & 16 & 3.1 & .6 & 1.8 & 1.6 \\
\hline Copper & 16 & 102 & 34.1 & 49.6 & 42.2 \\
\hline Iron & 16 & 2,310 & 472 & 1,140 & 1,010 \\
\hline Lead & 16 & 12.2 & 3.0 & 6.1 & 5.4 \\
\hline Manganese & 16 & 1,510 & 454 & 756 & 672 \\
\hline Nickel & 16 & 1.9 & .6 & 1.1 & 1.0 \\
\hline Zinc & 16 & 235 & 145 & 192 & 183 \\
\hline \multicolumn{6}{|c|}{ Hydropsyche spp. } \\
\hline Cadmium & 1 & - & -- & 1.3 & -- \\
\hline Chromium & 1 & - & -- & 2.4 & - \\
\hline Copper & 1 & - & - & 84.1 & - \\
\hline Iron & 1 & -- & - & 1,800 & -- \\
\hline Lead & 1 & -- & -- & $<7.8$ & - \\
\hline Manganese & 1 & - & -- & 537 & - \\
\hline Nickel & 1 & - & - & 1.3 & - \\
\hline Zinc & 1 & - & -- & 171 & - \\
\hline \multicolumn{6}{|c|}{$\begin{array}{l}\text { 12340000--BLACKFOOT RIVER NEAR BONNER, MONT. } \\
\text { Period of record for biological data: } 1986-87,1991,1993,1996,1998\end{array}$} \\
\hline \multicolumn{6}{|c|}{ Arctopsyche grandis } \\
\hline Cadmium & 9 & .3 & $<.1$ & ${ }^{1} .2$ & ${ }^{1} .2$ \\
\hline Chromium & 3 & 1.8 & 1.2 & 1.4 & 1.3 \\
\hline Copper & 9 & 13.4 & 9.9 & 11.8 & 12.0 \\
\hline Iron & 9 & 1,230 & 108 & 596 & 617 \\
\hline Lead & 9 & 2.1 & .5 & 1.2 & .9 \\
\hline Manganese & 3 & 517 & 389 & 435 & 398 \\
\hline Nickel & 3 & 1.2 & .8 & 1.0 & 9 \\
\hline Zinc & 9 & 143 & 123 & 134 & 135 \\
\hline
\end{tabular}


Table 24. Statistical summary of biological data for the upper Clark Fork basin, Montana, August 1986 through August 1999 (Continued)

\begin{tabular}{|c|c|c|c|c|c|}
\hline & $\begin{array}{c}\text { Number of } \\
\text { composite } \\
\text { samples }\end{array}$ & Maximum & Minimum & Mean & Median \\
\hline \multicolumn{6}{|c|}{$\frac{12340000-\text {-BLACKFOOT RIVER NEAR BONNER, MONT.--Continued }}{\text { Period of record for biological data: } 1986-87,1991,1993,1996,1998}$} \\
\hline \multicolumn{6}{|c|}{ Claassenia sabulosa } \\
\hline Cadmium & 9 & .2 & .1 & .1 & .1 \\
\hline Chromium & 3 & .9 & .5 & .7 & .7 \\
\hline Copper & 9 & 88.5 & 19.0 & 44.5 & 41.0 \\
\hline Iron & 9 & 199 & 68.0 & 124 & 114 \\
\hline Lead & 9 & .6 & .4 & .5 & .6 \\
\hline Manganese & 3 & 127 & 44.2 & 74.6 & 52.6 \\
\hline Nickel & 3 & 3 & .1 & .2 & .2 \\
\hline Zinc & 9 & 329 & 117 & 204 & 194 \\
\hline \multicolumn{6}{|c|}{ Hydropsyche occidentalis } \\
\hline Cadmium & 12 & .5 & .1 & .2 & .2 \\
\hline Chromium & 12 & 2.7 & .8 & 1.8 & 1.7 \\
\hline Copper & 12 & 20.6 & 12.0 & 14.6 & 14.4 \\
\hline Iron & 12 & 1,930 & 1,050 & 1,410 & 1,380 \\
\hline Lead & 12 & 1.9 & .8 & 1.3 & 1.2 \\
\hline Manganese & 12 & 527 & 414 & 472 & 460 \\
\hline Nickel & 12 & 1.8 & 9 & 1.3 & 1.2 \\
\hline Zinc & 12 & 150 & 117 & 134 & 130 \\
\hline \multicolumn{6}{|c|}{ Hydropsyche spp. } \\
\hline Cadmium & 1 & -- & - & .6 & -- \\
\hline Chromium & 1 & - & -- & 1.6 & - \\
\hline Copper & 1 & -- & - & 13.9 & -- \\
\hline Iron & 1 & - & - & 1,120 & - \\
\hline Lead & 1 & -- & $\sim$ & 2.9 & - \\
\hline Manganese & 1 & - & - & 525 & -- \\
\hline Nickel & 1 & -- & - & 28 & -- \\
\hline Zinc & 1 & - & - & 132 & - \\
\hline
\end{tabular}


Table 24. Statistical summary of biological data for the upper Clark Fork basin, Montana, August 1986 through August 1999 (Continued)

\begin{tabular}{|c|c|c|c|c|c|}
\hline Constituent & $\begin{array}{c}\text { Number of } \\
\text { composite } \\
\text { samples }\end{array}$ & Maximum & Minimum & Mean & Median \\
\hline \multicolumn{6}{|c|}{$\frac{12340500-C L A R K \text { FORK ABOVE MISSOULA, MONT. }}{\text { Period of record for biological data: } 1997-99}$} \\
\hline \multicolumn{6}{|c|}{ Arctopsyche grandis } \\
\hline Cadmium & 11 & 1.8 & .5 & .9 & .8 \\
\hline Chromium & 11 & 3.0 & 1.3 & 1.7 & 1.7 \\
\hline Copper & 11 & 77.6 & 22.3 & 36.9 & 36.8 \\
\hline Iron & II & 2,340 & 708 & 1,160 & 1,050 \\
\hline Lead & 11 & 6.8 & 1.2 & 3.3 & 3.3 \\
\hline Manganese & 11 & 1,040 & 476 & 779 & 782 \\
\hline Nickel & 11 & 2.0 & .7 & 1.2 & 1.2 \\
\hline Zinc & 11 & 235 & 155 & 187 & 177 \\
\hline \multicolumn{6}{|c|}{ Claassenia sabulosa } \\
\hline Cadmium & 4 & 2.0 & .6 & 1.3 & 1.3 \\
\hline Chromium & 4 & 1.1 & .4 & .8 & .9 \\
\hline Copper & 4 & 61.9 & 37.8 & 47.9 & 46.0 \\
\hline Iron & 4 & 246 & 95.0 & 167 & 163 \\
\hline Lead & 4 & 3.1 & .5 & ${ }^{1} 1.4$ & ${ }^{1} .5$ \\
\hline Manganese & 4 & 136 & 75.2 & 107 & 109 \\
\hline Nickel & 4 & $<1.3$ & $<.3$ & $1-$ & $1<.7$ \\
\hline Zinc & 4 & 307 & 191 & 250 & 250 \\
\hline \multicolumn{6}{|c|}{ Hydropsyche cockerelli } \\
\hline Cadmium & 6 & 1.3 & .8 & 1.0 & 1.0 \\
\hline Chromium & 6 & 4.1 & 2.1 & 3.2 & 3.2 \\
\hline Copper & 6 & 96.1 & 40.4 & 66.6 & 64.7 \\
\hline Iron & 6 & 3,590 & 1,900 & 2,450 & 2,250 \\
\hline Lead & 6 & 6.3 & 5.3 & 5.8 & 5.9 \\
\hline Manganese & 6 & 1,180 & 781 & 930 & 904 \\
\hline Nickel & 6 & 2.4 & 1.4 & 2.0 & 2.1 \\
\hline Zinc & 6 & 226 & 156 & 199 & 200 \\
\hline \multicolumn{6}{|c|}{ Hydropsyche occidentalis } \\
\hline Cadmium & 4 & 1.1 & .7 & .9 & .9 \\
\hline Chromium & 4 & 3.2 & 2.8 & 3.0 & 3.0 \\
\hline Copper & 4 & 76.5 & 46.5 & 57.2 & 52.9 \\
\hline Iron & 4 & 2,400 & 2,010 & 2,220 & 2,220 \\
\hline Lead & 4 & 7.7 & 5.0 & 6.5 & 6.6 \\
\hline Manganese & 4 & 2,460 & 939 & 1,930 & 2,160 \\
\hline Nickel & 4 & 2.3 & 1.9 & 2.1 & 2.2 \\
\hline Zinc & 4 & 232 & 210 & 225 & 230 \\
\hline \multicolumn{6}{|c|}{$\frac{12353000 \text {-CLARK FORK BELOW MISSOULA, MONT. }}{2}$} \\
\hline \multicolumn{6}{|c|}{ Arctopsyche grandis } \\
\hline Cadmium & 20 & 1.5 & .3 & .8 & .8 \\
\hline Chromium & 20 & 2.7 & .5 & 1.4 & 1.4 \\
\hline Copper & 20 & 38.0 & 9.4 & 22.0 & 20.3 \\
\hline lron & 20 & 1,500 & 343 & 810 & 832 \\
\hline Lead & 20 & 3.2 & .9 & 1.9 & 1.9 \\
\hline Manganese & 20 & 1,090 & 511 & 713 & 710 \\
\hline Nickel & 20 & 1.6 & .4 & .9 & .9 \\
\hline \multirow[t]{2}{*}{ Zinc } & 20 & 217 & 106 & 154 & 148 \\
\hline & 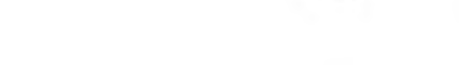 & & & 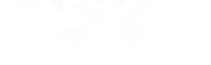 & TA \\
\hline
\end{tabular}


Table 24. Statistical summary of biological data for the upper Clark Fork basin, Montana, August 1986 through August 1999 (Continued)

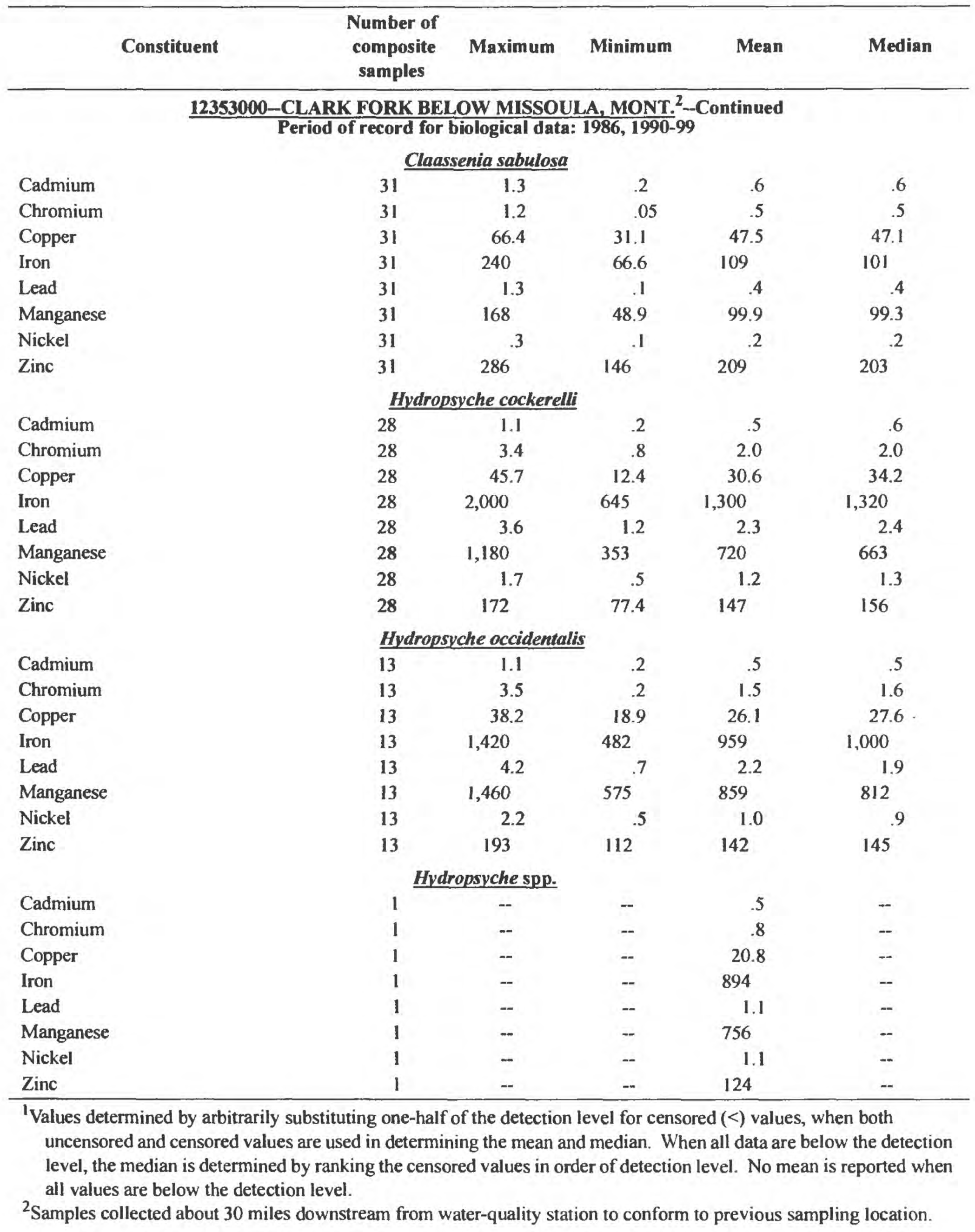

